\title{
Representações de Álgebras Correntes
}

\author{
Henrique de Oliveira Rocha \\ DISSERTAÇÃO APRESENTADA \\ AO \\ Instituto DE MATEMÁticA E EstatísticA \\ DA \\ UNIVERSIDADE DE SÃo PAUlO \\ PARA \\ OBTENÇÃO DO TÍTULO \\ DE \\ Mestre em CiÊnCIAS \\ Programa: Matemática \\ Orientador: Prof. Dr. Vyacheslav Futorny
}

Durante o desenvolvimento deste trabalho o autor recebeu auxílio financeiro do $\mathrm{CNPq}$ (122692/2017-9) e FAPESP (2018/12382-9)

São Paulo, Dezembro de 2020 


\section{Representações de Álgebras Correntes}

Esta versão da dissertação/tese contém as correções e alterações sugeridas pela Comissão Julgadora durante a defesa da versão original do trabalho, realizada em 02/12/2020. Uma cópia da versão original está disponível no Instituto de Matemática e Estatística da Universidade de São Paulo.

Comissão Julgadora:

- Prof. Dr. Vyacheslav Futorny (orientador) - IME-USP

- Prof. Dr. Lucas Henrique Calixto - UFMG

- Prof. Dr. Angelo Calil Bianchi - UNIFESP 


\section{Resumo}

ROCHA, H. O. Representações de Álgebras Correntes. 2020. 116 f. Dissertação (Mestrado) - Instituto de Matemática e Estatística, Universidade de São Paulo, São Paulo, 2020.

O primeiro objetivo desta dissertação é estudar os $\mathcal{G}$-módulos de peso simples cujos espaços de peso possuem dimensão finita, onde $\mathcal{G}=\mathfrak{g} \otimes A, \mathfrak{g}$ é uma álgebra de Lie redutível de dimensão finita e $A$ é uma álgebra comutativa associativa com unidade e finitamente gerada. Em particular, mostraremos que tais módulos podem ser descritos a partir de módulos de avaliação e módulos de indução parabólica. O segundo objetivo é estudar subálgebras comutativas de $U\left(\mathfrak{g}_{m}(n)\right)$, onde $\mathfrak{g}_{m}(n)=\mathfrak{g l}_{n}(\mathbb{C}) \otimes\left(\mathbb{C}[t] /\left\langle t^{m}\right\rangle\right)$. Para $n \leq 3$ ou $m \leq 2$, mostraremos que a imagem graduada de determinados geradores algebricamente independentes do centro de $U\left(\mathfrak{g}_{m}(n)\right)$ formam uma sequência regular da álgebra graduada associada à $U\left(\mathfrak{g}_{m}(n)\right)$. Também mostraremos que, para $m>1$, a imagem graduada dos geradores da subálgebra de Gelfand-Tsetlin de $U\left(\mathfrak{g}_{m}(n)\right)$ formam uma sequência regular se, e somente se, $n=1$ ou $n=2$. Por fim, mostraremos que a imagem graduada dos geradores da subálgebra de Bethe formam uma sequência regular, se $n=2$ e $m \geq 1$ ou $n=3$ e $m=1$. Estes resultados implicam a liberdade de $U\left(\mathfrak{g}_{m}(n)\right)$ como módulo sobre tais subálgebras comutativas, além da existência de $\mathfrak{g}_{m}(n)$-módulos irredutíveis levantados de tais álgebras.

Palavras-chave: álgebras correntes, teoria das representações, sequências regulares. 


\section{Abstract}

ROCHA, H. O. Representations of Current Algebras. 2020. 116 p. Dissertação (Mestrado) Instituto de Matemática e Estatística, Universidade de São Paulo, São Paulo, 2020.

The first objective of this thesis is to study simple weight $\mathcal{G}$-modules with finite dimensional weight spaces, where $\mathcal{G}=\mathfrak{g} \otimes A, \mathfrak{g}$ is a reductive Lie algebra with finite dimension and $A$ is an associative unital commutative algebra of finite type. In particular we show that such modules are given by evaluation modules and parabolic induced modules. The second objective is study commutative algebras of $U\left(\mathfrak{g}_{m}(n)\right)$, where $\mathfrak{g}_{m}(n)=\mathfrak{g l}_{n}(\mathbb{C}) \otimes\left(\mathbb{C}[t] /\left\langle t^{m}\right\rangle\right)$. For $n \leq 3$ or $m \leq 2$, we will show the graded image of some algebraic independent generators of the center of $U\left(\mathfrak{g}_{m}(n)\right)$ form a regular sequence in the associated graded algebra. We will also prove that for $m>1$ the graded image of generators of the Gelfand-Tsetlin subalgebra of $U\left(\mathfrak{g}_{m}(n)\right)$ form a regular sequence if and only if $n=1$ or $n=2$. Finally, we will show the graded image of generators of the Bethe subalgebra form a regular sequence, if $n=2$ and $m \geq 1$ or $n=3$ and $m=1$. These results implies the freeness of $\left.U\left(\mathfrak{g}_{m}(n)\right)\right)$ over such commutative subalgebras and the existence of irreducible $\mathfrak{g}_{m}(n)$-modules lifted by such algebras.

Keywords: current algebras, representation theory, regular sequences. 


\section{Sumário}

$\begin{array}{ll}\text { Introdução } & 1\end{array}$

1 Conceitos Básicos e Preliminares $\quad 5$

1.1 Álgebras Filtradas, Álgebras Graduadas e Álgebras Comutativas . . . . . . . . . . . 5

1.2 Álgebras de Lie . . . . . . . . . . . . . . . . . . . . . . . . . . . . . . . . . . . . . . . . .

1.2.1 Extensões Centrais de Álgebras de Lie . . . . . . . . . . . . . . . . . . . 12

1.2.2 Classificação das Álgebras de Lie Semissimples . . . . . . . . . . . . . . . . . 14

1.2.3 Conjuntos Parabólicos nos Sistemas de Raízes . . . . . . . . . . . . . . . . . . 18

1.3 Representações de Álgebras de Lie . . . . . . . . . . . . . . . . . . . . . . . . . . . . . . . . . . . . . . . . .

1.3.1 Álgebra Universal Envolvente . . . . . . . . . . . . . . . . . . . . . 23

1.3.2 Representações de Peso . . . . . . . . . . . . . . . . . . . . . . . 29

1.4 Representações de Álgebras de Lie Semissimples . . . . . . . . . . . . . . . . . . . . 33

1.4.1 Módulos de Dimensão Finita e Módulos de Peso Máximo . . . . . . . . . . . 34

1.4 .2 Módulos de Verma . . . . . . . . . . . . . . . . . . . . . . 36

1.5 Variedades Algébricas e Teorema de Kostant . . . . . . . . . . . . . . . . 38

2 Álgebras Correntes e Módulos de Harish-Chandra $\quad 45$

2.1 Álgebras Correntes, Módulos de peso e Extensões Centrais . . . . . . . . . . . . . . . 46

2.1 .1 O Núcleo da Representação . . . . . . . . . . . . . . . . . . . . . . . . . . . 51

2.2 Módulos de Harish-Chandra e Indução Parabólica . . . . . . . . . . . . . . . . . . . 54

2.3 Módulos de Avaliação . . . . . . . . . . . . . . . . . . . . . . . . . . 62

2.4 Módulos Uniformemente Limitados Simples . . . . . . . . . . . . . . . . . . 67

2.5 Módulos de Avaliação de Módulos de Indução Parabólica . . . . . . . . . . . . . . . 73

2.6 Módulos de Peso Máximo . . . . . . . . . . . . . . . . . . . . . . 78

3 Variedades para Álgebra de Takiff Generalizada $\quad \mathbf{8 3}$

3.1 Geradores Algebricamente Independentes do Centro . . . . . . . . . . . . . . 84

3.2 Teorema de Kostant . . . . . . . . . . . . . . . . . . . . . . . . . . . . 89

3.2.1 Teorema de Kostant para $U\left(\mathfrak{g}_{m}(3)\right) \ldots \ldots \ldots \ldots$. . . . . . . 92

3.3 Subálgebra de Gelfand-Tsetlin . . . . . . . . . . . . . . . . . . . . . . 103

3.4 Subálgebra de Bethe . . . . . . . . . . . . . . . . . 106

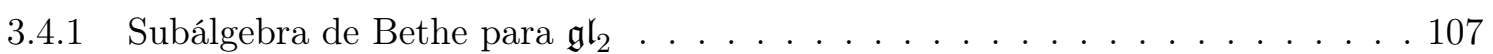

3.4.2 Subálgebra de Bethe para $\mathfrak{g l}_{3} \ldots \ldots \ldots$. . . . . . . . . . . 109

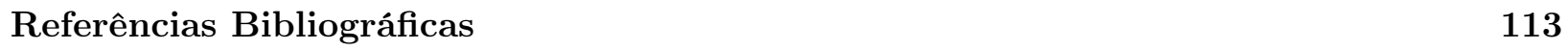




\section{Introdução}

Seja $\mathfrak{g}$ uma álgebra de Lie de dimensão finita e $A$ uma álgebra comutativa associativa com unidade, ambas sobre um corpo $\mathbb{k}$ algebricamente fechado de característica 0 . O espaço vetorial $\mathcal{G}=\mathfrak{g} \otimes A$ é uma álgebra de Lie, onde o colchete de Lie de $\mathcal{G}$ é dado por

$$
[x \otimes a, y \otimes b]=[x, y] \otimes a b \quad x, y \in \mathfrak{g}, \quad a, b \in A .
$$

A álgebra de Lie $\mathcal{G}$ é chamada de álgebra corrente (generalizada). Como mostrado em [NSS12] e [Lau14], em alguns casos, $\mathcal{G}$ é isomorfa à álgebra de Lie definida pontualmente no conjunto de funções regulares da forma $\operatorname{Spec}(A) \rightarrow \mathfrak{g}$ (equivalentemente, o conjunto dos morfismos de esquemas da forma $\operatorname{Spec}(A) \rightarrow \mathfrak{g})$, onde $\operatorname{Spec}(A)$ é o conjunto dos ideais primos de $A$.

O termo álgebra corrente apareceu no começo da década de 1960 a partir de trabalhos de Gell-Man ( [GM62, GM64]) para dar nome a uma álgebra que descrevia correntes fracas e eletromagnéticas de hádrons. Esta álgebra foi utilizada para provar resultados importantes na física e inspirou matemáticos e físicos a desenvolver a teoria de outras álgebras de Lie, como o modelo de Sugawara, álgebra de Virasoro e as álgebras de Kac-Moody afins. Com o desenvolvimento da teoria quânticas dos campos, o termo começou a ser utilizado para se referir às álgebras de Lie afins, que podem ser construídas como uma extensão central da álgebra de loop $\mathfrak{g} \otimes \mathbb{C}\left[t, t^{-1}\right]$, onde $\mathfrak{g}$ é uma álgebra de Lie simples. A álgebra de loop pode ser vista como a álgebra de Lie formada pelas aplicações suaves de $S^{1}$ para $\mathfrak{g}$. Isto inspirou a considerar álgebras de Lie que são formadas das aplicações suaves de uma variedade $M$ para uma álgebra de Lie de dimensão finita $\mathfrak{g}$, o que motivou a segunda (e terceira) definição de álgebras correntes dada no primeiro parágrafo. Para mais informações, leia as introduções dos livros [TJG72, Mic89].

Consideradas da forma como definimos, as álgebras correntes representam uma grande variedade de álgebras de Lie. O estudo das álgebras correntes, suas representações e aplicações pode ser encontrado extensivamente na literatura. Além das álgebras de loop $\mathfrak{g} \otimes \mathbb{C}\left[t, t^{-1}\right]$ ( $[$ Cha86, CP86, $\mathrm{CP} 87, \mathrm{CP} 88])$, temos as álgebras de multiloop $\mathfrak{g} \otimes \mathbb{C}\left[t_{1}^{ \pm}, t_{2}^{ \pm}, \ldots, t_{s}^{ \pm}\right]([$ER01, Lau10, PB10]), álgebras polinomiais corrente $\mathfrak{g} \otimes \mathbb{C}[t]([\mathrm{CG} 07, \mathrm{CG} 05])$, álgebras multicorrente polinomiais $\mathfrak{g} \otimes \mathbb{C}\left[t_{1}, t_{2}, \ldots, t_{k}\right]$ e seus quocientes ( [MS19]), álgebras de Takiff (generalizadas) $\mathfrak{g} \otimes \mathbb{C}[t] /\left\langle t^{k}\right\rangle$ onde $k>1$ e $\left\langle t^{k}\right\rangle$ denota o ideal de $\mathbb{C}[t]$ gerado por $t^{k}$ ( [Tak71, RT92, Geo94a, Geo94b, Geo95, Wil11]), álgebra de Lie tetraedro $\mathfrak{s l}_{2} \otimes \mathbb{C}\left[t, t^{-1},(t-1)^{-1}\right]$ ( [Har07]), álgebras de $n$-ponto $\mathfrak{g} \otimes A$ (onde $A$ é álgebra de funções regulares de $X \rightarrow \mathfrak{g}$ com $X$ sendo a esfera de Riemman sem $n$ distintos pontos) ( [Bre95]), álgebras correntes de Krichever-Novikov $\mathfrak{g} \otimes \mathcal{A}$ (onde $\mathcal{A}$ é a álgebra de funções meromorfas em uma superfície de Riemman $\Sigma$ que são holomorfas fora de um conjunto finito $P \subset \Sigma)([\mathrm{KN} 87])$, entre outras.

Suponha que $\mathfrak{g}$ seja uma álgebra de Lie redutível e que $A$ é finitamente gerada. Todos os exemplos dados no parágrafo anterior podem ser adaptados para essas hipóteses. A classificação das representações irredutíveis de $\mathfrak{g}$ pode ser um problema extremamente difícil e foi feita apenas para $\mathfrak{g}=\mathfrak{s l}_{2}$ em [Blo81]. Portanto, o problema foi reduzido a classes menores de módulos ao longo do anos. Entre estas, podemos considerar os chamados módulos de peso, g-módulos tais que a ação de uma subálgebra de Cartan $\mathfrak{h} \subset \mathfrak{g}$ é diagonalizável. Porém, este problema também se mostrou muito complexo e ainda não foi finalizado. Sendo assim, condições de finitude foram impostas. Em [Mat00], Mathieu classificou todos os módulos de peso irredutíveis cujos espaços de peso possuem dimensão finita, consolidando e utilizando trabalhos de Fernando, Britten, Lemire, entre outros. 
A representação adjunta de $\mathfrak{h} \otimes 1 \cong \mathfrak{h}$ em $\mathcal{G}$ determina uma decomposição em espaços de peso, onde os pesos são dados por $\Phi \cup\{0\} \operatorname{com} \Phi$ sendo o sistema de raízes de $\mathfrak{g}$ associado à $\mathfrak{h}$. Isto nos permite considerar módulos de peso sobre $\mathcal{G}$, estes são $\mathcal{G}$-módulos tais que a ação de $\mathfrak{h}$ é diagonalizável. Um $\mathcal{G}$-módulo de peso simples cujos espaços de peso possuem dimensão finita será chamado de módulo de Harish-Chandra. Este nome é inspirado ao dado por Mathieu para módulos similares sobre a álgebra de Virasoro (veja [Mat92]). O estudo dos módulo de Harish-Chandra sobre $\mathcal{G}$ em termos de $\mathfrak{g}$-módulos é um dos nossos objetivos nesta dissertação.

O primeiro capítulo desta dissertação será focado em definir os objetos de estudo e exibir alguns resultados que precisaremos nos outros dois capítulos. Além de enunciar e referenciar teoremas clássicos da teoria de álgebra comutativa, geometria algébrica, álgebra de Lie e suas representações, demonstraremos alguns resultados que utilizaremos nos capítulos subsequentes. Em especial, destacamos os seguintes resultados.

Teorema. 1. (Proposição 1.3.22) Sejam $\mathfrak{g}_{1}$ e $\mathfrak{g}_{2}$ álgebras de Lie. Sejam $V_{1}$ e $V_{2}$ módulos simples sobre $\mathfrak{g}_{1}$ e $\mathfrak{g}_{2}$, respectivamente. Se $V_{1}$ possui dimensão enumerável, então $V_{1} \otimes V_{2}$ é um $\mathfrak{g}_{1} \oplus \mathfrak{g}_{2}$ módulo simples, onde a ação é induzida por

$$
\left(x_{1}+x_{2}\right)\left(v_{1} \otimes v_{2}\right)=\left(x_{1} v_{1}\right) \otimes v_{2}+x_{1} \otimes\left(x_{2} v_{2}\right), \quad x_{1} \in \mathfrak{g}_{1}, x_{2} \in \mathfrak{g}_{2}, v_{1} \otimes v_{2} \in V_{1} \otimes V_{2} .
$$

2. (Proposição 1.3.34) Sejam $\mathfrak{g}_{1}$ e $\mathfrak{g}_{2}$ álgebras de Lie que admitem uma decomposição em espaços de peso sobre subálgebras de Lie abelianas não nulas de dimensão finita $\mathfrak{h}_{1}$ e $\mathfrak{h}_{2}$, respectivamente. Suponha que $V$ seja um módulo quasi-finito sobre $\mathfrak{g}_{1} \oplus \mathfrak{g}_{2}$, isto é, $V$ é um $\mathfrak{g}_{1} \oplus \mathfrak{g}_{2}$-módulo de dimensão enumerável que admite uma decomposição em espaços de peso em relação a $\mathfrak{h}_{1} \oplus \mathfrak{h}_{2}$ e os espaços de peso possuem dimensão finita. Então $V \cong V_{1} \otimes V_{2}$, onde $V_{1}$ e $V_{2}$ são módulos quasi-finitos simples sobre $\mathfrak{g}_{1}$ e $\mathfrak{g}_{2}$, respectivamente.

Este teorema permite que suponhamos que $\mathfrak{g}$ é uma álgebra de Lie simples quando estudarmos os $\mathcal{G}$-módulos de Harish-Chandra e estender os resultados obtidos para o caso em que g é redutível, o que faremos no segundo capítulo. Suponha que g é simples. Em [Kas84], foi construído a extensão central universal de $\mathcal{G}$. Neste caso, a extensão central universal $\tilde{\mathcal{G}}$ de $\mathcal{G}$ e suas representações também possuem grande importância, como comentamos, as álgebras de Lie afins, por exemplo, são extensões centrais das álgebras de loop. Mostraremos, utilizando um argumento de traço feito em [BLL15], que a parte central de $\tilde{\mathcal{G}}$ age trivialmente em todo $\tilde{\mathcal{G}}$-módulo de Harish-Chandra, portanto todos os resultados obtidos para $\mathcal{G}$-módulos de Harish-Chandra podem ser estendidos para $\tilde{\mathcal{G}}$.

O estudo dos $\mathcal{G}$-módulos de Harish-Chandra para $\mathfrak{g}$ simples e $A$ finitamente gerada dada no segundo capítulo desta dissertação será dada em termos de g-módulos. Para isto, definiremos os módulos de avaliação: $\mathcal{G}$-módulos construídos a partir de $\mathfrak{g}$-módulos e ideais maximais distintos de $A$. Mostraremos quais módulos de avaliação são uniformemente limitados - $\mathcal{G}$-módulos tais que a dimensão dos espaços de peso é limitada por algum número positivo. Em particular, demonstraremos que um módulo de avaliação definido a partir de g-módulos de peso simples é uniformemente limitado se, e somente se, no máximo um dos g-módulos tomados possui dimensão infinita (Teorema 2.4.4). Também mostraremos que todo $\mathcal{G}$-módulo uniformemente limitado é um módulo de avaliação (Teorema 2.4.7). Mostraremos que módulos de avaliação construídos a partir de ideais maximais distintos de $A$ e módulos de indução parabólica definidos a partir do mesmo conjunto parabólico são $\mathcal{G}$-módulos de Harish-Chandra (Proposição 2.5.6). Todas essas proposições e teoremas foram provados em [BLL15, Lau18], porém os demonstraremos de forma mais detalhada. Terminaremos o segundo capítulo desta dissertação aplicando estes resultados à teoria de $\mathcal{G}$-módulos de peso máximo. Mostraremos que $L(\Lambda)$ é um módulo de Harish-Chandra se, e somente se, existe um ideal $I$ de $A$ tal que $\Lambda(\mathfrak{h} \otimes I)=0$. Além disso, mostraremos a seguinte proposição.

Proposição (Proposição 2.6.8). Seja $\Lambda \in(\mathfrak{h} \otimes A)^{*}$. Se existem funcionais lineares $\lambda_{1}, \ldots, \lambda_{r} \in \mathfrak{h}^{*}$ e ideais maximais distintos $m_{1}, \ldots, m_{r}$ de $A$ tais que $\Lambda(h \otimes a)=\sum_{i=1}^{r} a\left(m_{i}\right) \lambda_{i}(h)$, para todo $h \in \mathfrak{h}$ 
e a $\in$ A, então $L(\Lambda)$ é um $\mathcal{G}$-módulo de Harish-Chandra e

$$
L(\Lambda) \cong \bigotimes_{i=1}^{r} L\left(\lambda_{i}\right)\left(m_{i}\right),
$$

onde $L\left(\lambda_{i}\right)$ é o $\mathfrak{g}$-módulo simples de peso máximo $\lambda_{i}$ para cada $i=1, \ldots, r$.

O terceiro capítulo terá uma abordagem bem diferente do anterior. Nosso objetivo é perguntar sobre a relação de $U(\mathcal{G})$ com suas subálgebras comutativas, pois a ação de subálgebras comutativas de $U(\mathfrak{g})$ em $\mathfrak{g}$-módulos já implicou diversos resultados importantes. Por exemplo, a ação do centro de $U(\mathfrak{g})$ em $\mathfrak{g}$-módulos de dimensão finita, através do Teorema de Harish-Chandra, pode ser aplicada para a obtenção da fórmula de Weyl, que determina quais sãos as dimensões dos g-módulos de dimensão finita. Outro exemplo é encontrado na classificação de $\mathfrak{s l}_{2}$-módulos, que envolve parâmetros correspondentes aos autovalores dos geradores da subálgebra de Gelfand-Tsetlin de $U\left(\mathfrak{s l}_{2}\right)$. Em [FO05], mostrou-se que, se $\mathcal{G}$ possui dimensão finita e $\mathcal{A}$ é uma subálgebra comutativa de $U(\mathcal{G})$ gerada por elementos algebricamente independentes cujas imagens graduadas formam uma sequência regular da álgebra graduada $\bar{U}(\mathcal{G})$ associada à filtração de $U(\mathcal{G})$ dada pelo Teorema de Birkhoff-Poincaré-Witt, então

1. [FO05, Theorem 1.1] $U(\mathcal{G})$ é livre como um módulo sobre $\mathcal{A}$;

2. [FO05, Proposition 3.4] existe um $U(\mathcal{G})$-módulo irredutível $M_{\nu}$ com gerador $m$ tal que $g m=$ $\chi_{\nu}(g) m$ para todo $g \in \mathcal{A}$, onde $\chi_{\nu}: \mathcal{A} \rightarrow \mathbb{k}$ é um carácter associado ao ideal maximal $\nu$ de $\mathcal{A}$.

Portanto, consideraremos geradores algebricamente independentes do centro de $U\left(\mathfrak{g}_{m}(n)\right)$ construídos em [Mol97], onde $\mathfrak{g}_{m}(n)=\mathfrak{g l}_{n} \otimes\left(\mathbb{C}[t] /\left(t^{m}\right)\right)$. Para $m \leq 2$ ou $n \leq 3$, mostraremos que a imagem graduada de tais geradores formam uma sequência regular em $\bar{U}\left(\mathfrak{g}_{m}(n)\right)$ (Teorema 3.2.3, Proposição 3.2.5, Corolário 3.2.13). Pelo [FO05, Theorem 1.1], temos que

Teorema. $U\left(\mathfrak{g}_{m}(n)\right)$ é livre como um módulo sobre seu centro para $m \leq 2$ ou $n \leq 3$.

Utilizaremos tais geradores construídos para considerar também a subálgebra de Gelfand-Tsetlin de $U\left(\mathfrak{g}_{m}(n)\right)$. Estudar a regularidade da imagem graduada de tais geradores será equivalente a calcular a dimensão das componentes irredutíveis da variedade de Gelfand-Tsetlin $\mathcal{V G \mathcal { G }}_{m}(n)$. Para o caso $m=1$, foi provado em [Ovs03] que $\mathcal{V G} \mathcal{T}_{1}(n)$ é equidimensional de dimensão $(n-$ 1) $n / 2$. Portanto, é natural conjecturar que $\mathcal{V G} \mathcal{T}_{m}(n)$ é equidimensional de dimensão $m n(n-1) / 2$. Mostraremos que isto não é verdade.

Teorema (Teorema 3.3.3). Para $m>1, \mathcal{V} \mathcal{G} \mathcal{T}_{m}(n)$ é equidimensional de dimensão $m n(n-1) / 2$ se e somente se $n=1$ ou $n=2$.

Para $m>1$, isto implicará que a sequência formada pela imagem graduada dos geradores $\Gamma_{m}(n)$ é regular se, e somente se, $n=1$ ou $n=2$. Aplicando [FO05, Theorem 1.1], temos que $U\left(\mathfrak{g}_{m}(2)\right)$ é livre como um módulo sobre subálgebra de Gelfand-Tsetlin para todo $m \geq 1$. Terminaremos o terceiro capítulo tentando utilizar os geradores da subálgebra de Bethe de $U\left(\mathfrak{g}_{m}(n)\right)$ determinados em [Tal06, MTV06] para mostrar que eles formam uma sequência regular em $\bar{U}\left(\mathfrak{g}_{m}(n)\right)$. Neste caso, conseguimos mostrar a regularidade apenas para $n=2$ e $m$ qualquer ou $n=3$ e $m=1$ (Teorema 3.4.2, Proposição 3.4.5).

Os resultados obtidos no terceiro capítulo desta dissertação foram desenvolvidos com a colaboração do Prof. Dr. Germán Benitez Monsalve e foram aceitos para publicação na São Paulo Journal of Mathematical Sciences, doi: 10.1007/s40863-020-00204-1. 


\section{Capítulo 1}

\section{Conceitos Básicos e Preliminares}

O principal objetivo deste capítulo é fixar a notação e expor alguns resultados que precisaremos nos capítulos subsequentes.

Começaremos o capítulo definindo e expondo alguns resultados da teoria de álgebras filtradas e álgebras comutativas na Seção 1.1. Esses resultados serão importantes no Capítulo 2. Em seguida, na Seção 1.2, definiremos e exporemos uma série de resultados da teoria das álgebras de Lie. Falaremos sobre extensões centrais e a classificação das álgebras de Lie semissimples, terminando a seção com alguns resultados sobre conjuntos parabólicos dentro dos sistemas de raízes que geralmente não são apresentados nos livros introdutórios às álgebras de Lie. A teoria desenvolvida nesta seção será importante durante todo este trabalho.

Na Seção 1.3, o assunto será voltado à teoria de representações das álgebras de Lie. Precisaremos de resultados clássicos da teoria, como o Lema de Schur e o Teorema de Poincaré-Birkhoff-Witt, para provar resultados sobre o produto tensorial de representações simples. Provaremos alguns resultados sobre a representação da soma direta de álgebras de Lie que admitem uma decomposição em espaços de peso de uma subálgebra abeliana não nula. Alguns destes resultados são generalizações de proposições e lemas dados em [BLL15]. Na Seção 1.4 exporemos um pouco da teoria de representações das álgebras de Lie semissimples. Em especial, falaremos sobre módulos de peso máximo, que possuem um papel fundamental nesta teoria.

No final do capítulo, na Seção 1.5, exporemos um pouco do básico da teoria de geometria algébrica e variedades algébricas. Além disso, apresentaremos uma técnica desenvolvida em [FO05] para mostrar que uma álgebra filtrada é livre como um módulo sobre uma subálgebra comutativa. Esta seção será mais importante Capítulo 3, porém também iremos utilizá-la para explicar alguns exemplos no Capítulo 2.

Neste capítulo, suporemos que $\mathbb{k}$ é um corpo algebricamente fechado de característica 0. Por exemplo, o leitor pode supor $\mathbb{k}=\mathbb{C}$.

\section{1 Álgebras Filtradas, Álgebras Graduadas e Álgebras Comutati- vas}

Notação 1.1.1. Durante toda esta dissertação, denotaremos por $\mathbb{Z}_{>0}$ o conjunto dos números inteiros estritamente positivos e por $\mathbb{Z}_{\geq 0}$ o conjunto dos números inteiros não negativos. Similarmente, definimos $\mathbb{Z}_{<0}$ e $\mathbb{Z}_{\leq 0}$.

Definição 1.1.2. Seja $U$ um espaço vetorial sobre $\mathbb{k}$. O par $(U, \cdot)$ é chamado de $\mathbb{k}$-álgebra, se $\cdot: U \times U \rightarrow U$ é uma transformação bilinear e, para todo $x, y, z \in A$ e para todo $\lambda \in \mathbb{k}$, temos que

$$
(\lambda x+y) \cdot z=\lambda(x \cdot z)+y \cdot z \mathrm{e} x \cdot(\lambda y+z)=\lambda(x \cdot y)+x \cdot y .
$$

Dizemos que a k-álgebra $(A, \cdot)$ é

(i) associativa, se para todo $x, y, z \in A,(x \cdot y) \cdot z=x \cdot(y \cdot z)$; 
(ii) com unidade, se existe $1_{A} \in \mathbb{k}$ tal que $1_{A} \cdot x=x \cdot 1_{A}=x$ para todo $x \in A$.

Quando não houver ambiguidade, vamos denotar por $x y$ o produto $x \cdot y$ para qualquer $x, y \in A$. Quando não houver dúvidas de qual aplicação $\cdot$, diremos apenas que $A$ é uma $k$-álgebra ou apenas álgebra.

Exemplo 1.1.3. Seja $V$ um espaço vetorial. Considere $U=\operatorname{End}(V)$ o espaço dos endomorfismos de $V$, então $U$ é uma k-espaço vetorial. A composição de endomorfismos o é uma aplicação bilinear associativa e a aplicação identidade $I$ é tal que $I \circ T=T \circ I=T$, para todo $T \in U$. Logo, $(\operatorname{End}(V)$, o $)$ é uma k-álgebra.

Se $V$ é um $\mathbb{k}$-espaço vetorial de dimensão finita e $\left\{e_{1}, \ldots, e_{n}\right\}$ é uma base de $V$, então existe um isomorfismo linear entre o espaço de endomorfismos de $V$, $\operatorname{End}(V)$, e o espaço vetorial das matrizes $n \times n, M_{n}(\mathbb{k})$. Este isomorfismo induz um produto bilinear em $M_{n}(\mathbb{k})$, que define uma estrutura de $\mathbb{k}$-álgebra associativa com unidade em $M_{n}(\mathbb{k})$. Da teoria de álgebra linear, sabemos que este produto bilinear é exatamente o produto de matrizes.

Exemplo 1.1.4. Considere o espaço vetorial dos polinômios em $n$ variáveis $\mathbb{k}\left[x_{1}, \ldots, x_{n}\right]$, então o produto usual de polinômios define uma estrutura de $\mathbb{k}$-álgebra associativa com unidade em $\mathbb{k}\left[x_{1}, \ldots, x_{n}\right]$.

Note que se $U$ é uma k-álgebra, então $U$ é um anel associativo com unidade. Como feito na teoria de anéis, podemos definir ideais (à esquerda e à direita), subálgebras e homomorfismos de álgebras, com o único comentários que estas definições devem ser compatíveis com a estrutura de espaço vetorial. Isto é, ideais e subálgebras serão subespaços vetoriais de $U$ e homomorfismos de álgebras serão transformações lineares. Além disso, resultados básicos da teoria de anéis, como o Teorema do Isomorfismo e o Teorema da Correspondência também valem para álgebras associativas com unidade.

Definição 1.1.5. Dizemos que uma álgebra $U$ associativa com unidade é uma álgebra graduada, se existir uma coleção de subespaços vetoriais $\left\{U_{n}\right\}_{n \in \mathbb{Z}_{>0}}$, tal que $U_{n} \cdot U_{m} \subseteq U_{n+m}$ e

$$
U=\bigoplus_{n=0}^{\infty} U_{n}
$$

Neste caso, dizemos que $\left\{U_{n} \mid n \in \mathbb{Z}_{\geq 0}\right\}$ é uma graduação de $A$. Além disso, os elementos de $U_{n}$ são chamados de homogêneos de grau $n$.

Observe que todo elemento $a \in U$ pode ser escrito de maneira única como soma $a=\sum_{n \in \mathbb{Z}_{\geq 0}} a_{n}$ de elementos homogêneos $a_{n} \in U_{n}$, então podemos definir $a_{n}$ como a componente homogênea de grau $n$ do elemento $a$ e o maior $n$ tal que $a_{n} \neq 0$ é chamado de grau de $a$.

Naturalmente, pode ser importante que homomorfismos entre álgebras graduadas respeitem suas respectivas graduações, por isso se faz necessário a próxima definição.

Definição 1.1.6. Sejam $A$ e $B$ duas álgebras graduadas com graduação $\left\{A_{n}\right\}_{n \in \mathbb{Z}_{\geq 0}}$ e $\left\{B_{n}\right\}_{n \in \mathbb{Z}_{\geq 0}}$, respectivamente. Um homomorfismo de álgebras graduadas (ou homomorfismo graduado) $\phi: A \rightarrow$ $B$ é um homomorfismo de álgebras tal que $\varphi\left(A_{n}\right) \subseteq B_{n}$, para todo $n \in \mathbb{Z}_{\geq 0}$.

Exemplo 1.1.7. A álgebra $\mathbb{k}\left[x_{1}, \ldots, x_{n}\right]$ de polinômios em $n$ variáveis é uma álgebra graduada com a graduação canônica

$$
\mathbb{k}\left[x_{1}, \ldots, x_{n}\right]=\bigoplus_{m \in \mathbb{Z}_{\geq 0}} \mathbb{k}\left[x_{1}, \ldots, x_{n}\right]_{m}
$$

em que $\mathbb{k}\left[x_{1}, \ldots, x_{n}\right]_{m}$ é o subespaço gerado pela base formada por monômios $x_{1}^{d_{1}} \ldots x_{n}^{d_{n}}$ de grau $m=d_{1}+\cdots+d_{n}$.

Evidentemente, $\mathbb{k}\left[x_{1}, \ldots, x_{n}\right]$ admite outras graduações. Por exemplo, denote por $\mathbb{k}\left[x_{1}, \ldots, x_{n}\right]^{1}$ a álgebra de polinômios em $n$ variáveis com a graduação $\mathbb{k}\left[x_{1}, \ldots, x_{n}\right]_{m}^{1}$, com base dada pelos 
monômios $x_{1}^{d_{1}} x_{2}^{d_{2}} \ldots x_{n}^{d_{n}}$ com $m=d_{1}+2 d_{2}+\cdots+n d_{n}$. Neste caso, a identidade $i: \mathbb{k}\left[x_{1}, \ldots, x_{n}\right]^{1} \rightarrow$ $\mathbb{k}\left[x_{1}, \ldots, x_{n}\right]$ não é um homomorfismo de álgebras graduadas, porém o homomorfismo injetor $\varphi$ : $\mathbb{k}\left[x_{1}, \ldots, x_{n}\right]^{1} \rightarrow \mathbb{k}\left[x_{1}, \ldots, x_{n}\right]$ definido por $\varphi\left(x_{1}^{d_{1}} x_{2}^{d_{2}} \ldots x_{n}^{d_{n}}\right)=x_{1}^{d_{1}} x_{2}^{2 d_{2}} \ldots x_{n}^{n d_{n}}$ é um homomorfismo graduado.

Definição 1.1.8. Seja $U$ uma k-álgebra associativa com unidade. Dizemos que $U$ é filtrada, se existir uma cadeia crescente de subespaços $F_{0}=\mathbb{k} \subseteq F_{1} \subseteq F_{2} \subseteq \cdots \subseteq F_{i} \subseteq \cdots \subseteq U$ tal que $F=\bigcup_{i=0}^{\infty} F_{i}$ e $F_{i} F_{j} \subseteq F_{i+j}$. Neste caso, dizemos que $\overline{\mathcal{F}}=\left\{F_{i} \mid i \in \mathbb{Z}_{\geq 0}\right\}$ é uma filtração para $U$.

A definição acima é parecida com a definição de álgebra graduada e, de fato, toda álgebra graduada admite uma filtração. Se $U$ é uma álgebra graduada com graduação $\left\{U_{n}\right\}_{n \in \mathbb{Z}_{\geq 0}}$, então

$$
F_{n}=\bigoplus_{i=0}^{n} U_{i}, \quad n \in \mathbb{Z}_{\geq 0}
$$

define uma filtração em $U$. Neste sentido, a álgebra filtrada é uma generalização da noção de álgebra graduada.

Por outro lado, se $U$ é uma álgebra filtrada, também podemos associar a ela uma álgebra graduada. Seja $U$ uma álgebra filtrada com filtração $\left\{F_{n}\right\}_{n \in \mathbb{Z}_{\geq 0}}$. Seja $F_{-1}=\{0\}$ e o quociente de espaços vetoriais dado por

$$
U_{(n)}=F_{n} / F_{n-1},
$$

com projeções canônicas $\pi_{n}: F_{n} \rightarrow U_{(n)}, n \in \mathbb{Z}_{\geq 0}$. Defina o espaço vetorial

$$
\operatorname{gr}(U)=\bigoplus_{n \in \mathbb{Z} \geq 0} U_{(n)}
$$

Para cada $x \in U_{(n)}$ e $y \in U_{(n)}$, tome $x^{\prime} \in F_{n}$ e $y^{\prime} \in F_{m}$ tais que $x=\pi_{n}\left(x^{\prime}\right)$ e $y=\pi_{m}\left(y^{\prime}\right)$. Então, podemos definir uma multiplicação em $\operatorname{gr}(A)$ dada por

$$
x y=x \bullet y=\pi_{n+m}\left(x^{\prime} y^{\prime}\right) .
$$

Como a projeção canônica está bem definida e $x^{\prime} y^{\prime} \in F_{n+m}$, temos que o produto está bem definido em $\operatorname{gr}(U)$. Note que $\left\{U_{(n)}\right\}_{n \in \mathbb{Z}_{\geq 0}}$ define uma graduação em $\operatorname{gr}(U)$, portanto $\operatorname{gr}(U)$ é uma álgebra graduada.

Definição 1.1.9. A álgebra $\operatorname{gr}(U)$ munida com o produto definido acima é chamada de álgebra graduada associada à filtração de $U$ ou quando a filtração está fixada, chamaremos apenas de graduação associada a $U$. Se a filtração $\left\{F_{n}\right\}_{n \in \mathbb{Z}_{\geq 0}}$ de $U$ está fixada, então denotaremos por $\bar{u} \in F_{n} / F_{n-1} \subset \operatorname{gr}(U)$ a imagem de $u$ em $\operatorname{gr}(U)$, se $u \in F_{n} \backslash F_{n-1}$. Além disso, se $S \subset U$, denotaremos por $\bar{S}=\{\bar{u} \mid u \in S\}$.

Observe que, se $x, y, z \in U$, então $\bar{x}(\bar{y} \bar{z})=\overline{x(y z)}=\overline{(x y) z}=(\bar{x} \bar{y}) \bar{z}$. Logo, se $U$ é associativa, então $\operatorname{gr}(U)$ também é. Além disso, podemos fazer o mesmo processo para verificar que se $U$ satisfaz alguma identidade bilinear, como ter unidade, comutatividade ou a identidade de Jacobi, então $\operatorname{gr}(U)$ também satisfaz.

Agora apresentaremos alguns resultados relacionados às álgebras comutativas. Iremos utilizar [AM69] como referência. Para um livro com explicações mais detalhadas, veja [Eis95]. Para um referência em português, veja [BT15].

Definição 1.1.10. Seja $A$ uma k-álgebra. Dizemos que $A$ é uma k-álgebra afim, se $A$ é uma álgebra comutativa, associativa com unidade finitamente gerada sobre $\mathbb{k}$. Isto é, $A$ é uma k-álgebra associativa com unidade tal que

(i) (comutatividade) $a b=b a$, para todo $a, b \in A$; 
(ii) (finitamente gerada) existem $a_{1}, \ldots, a_{n}$ tais que, para todo $a \in A$, existe um polinômio $p \in \mathbb{k}\left[x_{1}, \ldots, x_{n}\right]$ tal que $a=p\left(a_{1}, \ldots, a_{n}\right)$.

Como comentamos no caso de álgebras associativas com unidade, temos que $A$ é um anel associativo comutativo com unidade. Da mesma forma, podemos definir ideais, subálgebras e homomorfismos de álgebras, com o único comentário de que estas definições devem ser compatíveis com a estrutura de espaço vetorial. Isto é, ideais e subálgebras serão subespaços vetoriais de $A$ e homomorfismos de álgebras serão transformações lineares. Além disso, resultados básicos da teoria de anéis, como o Teorema do Isomorfismo e o Teorema da Correspondência também valem para álgebras afins.

Exemplo 1.1.11. O anel de polinômios em $n$ variáveis $\mathbb{k}\left[x_{1}, \ldots, x_{n}\right]$ é uma álgebra afim. Além disso, todo quociente $\mathbb{k}\left[x_{1}, \ldots, x_{n}\right] / I$ por um ideal $I$ é uma álgebra afim.

Teorema 1.1.12 (Teorema dos zeros de Hilbert). Seja A uma álgebra afim sobre o corpo algebricamente fechado $\mathbb{k}$.

(i) Se A é um corpo, então A possui dimensão finita.

(ii) $S e m \subset A$ é um ideal primo, então $M$ é maximal se, e somente se, $A / M$ possui dimensão finita, quando visto como espaço vetorial sobre $\mathbb{k}$. Como $\mathbb{k}$ é algebricamente fechado e $m$ é um ideal primo, temos que A/M possui dimensão finita se, e somente se, $A / M$ possui dimensão 1.

Demonstração. Veja Corollary 5.24 ou Proposition 7.9 de [AM69].

Como $A$ possui unidade, podemos ver $\mathbb{k} \subset A$. Além disso, note que a imagem de $\mathbb{k} \subset A \mathrm{em} A / m$ é sobrejetora para qualquer ideal maximal $m$. Assim, para todo $a \in A$ existe um único $\alpha \in \mathbb{k}$ tal que $a+m=\alpha+m$. A transformação linear $a \mapsto \alpha$ define um homomorfismo $A \mapsto \mathbb{k}$ de $k$-álgebras, chamado de aplicação de avaliação.

Teorema 1.1.13 (Teorema Chinês do Resto). Seja A uma álgebra afim e $I_{1}, \ldots, I_{k}$ ideais de $A$ tais que $I_{i}+I_{j}=A$ para $i \neq j$, então

(i) $I_{1} \cap \cdots \cap I_{k}=I_{1} \ldots I_{n}$;

(ii) A aplicação

$$
\begin{aligned}
\frac{A}{I_{1} \ldots I_{k}} & \rightarrow \frac{A}{I_{1}} \times \cdots \times \frac{A}{I_{k}} \\
a+I_{1} \ldots I_{k} & \mapsto\left(a+I_{1}, \ldots, a+I_{k}\right)
\end{aligned}
$$

é um isomorfismo de álgebras. Em particular, se $m_{1}, \ldots, m_{k}$ são ideais maximais distintos de $A$, então $\frac{A}{m_{1} \ldots m_{k}} \cong \mathbb{k}^{n}$.

Demonstração. Veja Proposition 1.10 de [AM69].

Definição 1.1.14. Seja $A$ uma álgebra afim e $I$ um ideal $A$, defina radical de $I$ como sendo

$$
\sqrt{I}=\left\{a \in I \mid a^{n} \in I \text { para algum } n \in \mathbb{Z}_{>0}\right\} .
$$

Dizemos que $I$ é um ideal radical se $I=\sqrt{I}$.

Todo ideal primo é radical. Além disso, se $a, b \in \sqrt{I}$ e $\lambda \in \mathbb{k}$, então

$$
(\lambda a+b)^{n}=\sum_{i=0}^{n}\left(\begin{array}{l}
n \\
k
\end{array}\right) \lambda^{n} a^{k} b^{n-k} .
$$

Logo, se tomarmos $n$ suficientemente grande, teremos que $\lambda a+b \in I$. Além disso, se $x \in A$, então $(x a)^{n}=x^{n} a^{n} \in I$ se $a^{n} \in I$. Assim, $x a \in \sqrt{I}$ também. Portanto, $\sqrt{I}$ é um ideal de $A$. 
Definição 1.1.15. Seja $A$ uma álgebra afim. Dizemos que $A$ é reduzida se $A$ não possui elementos nilpotentes. Isto é, $a^{n} \neq 0$ para todo $a \in A$ não nulo e $n \in \mathbb{Z}_{>0}$.

Se $A$ é uma álgebra afim e $I$ um ideal, então $I$ é radical se, e somente se, $A / I$ é uma álgebra afim reduzida.

Proposição 1.1.16. Seja A uma álgebra afim que possui dimensão finita, então A possui uma quantidade finita de ideais maximais e todo ideal primo de A é maximal. Além disso, o ideal (chamado ideal de Jacobson)

$$
\mathfrak{j}=\bigcap_{m \in \operatorname{Specm}(A)} m
$$

é nilpotente, onde $\operatorname{Specm}(A)$ denota o conjunto dos ideais maximais de A.

Demonstração. Como $A$ possui dimensão finita, temos que $A$ possui uma quantidade finita de ideais. Em particular, $A$ possui uma quantidade finita de ideais maximais. Se $I$ é um ideal primo de $A$, então $A / I$ possui dimensão finita. Pelo Teorema dos Zeros de Hilbert, $I$ é maximal. Logo, todo ideal primo de $A$ é maximal. Se j não é nilpotente, então é possível construir uma cadeia estritamente decrescente infinita de ideais, o que não é possível, visto que $A$ possui dimensão finita.

Corolário 1.1.17. Se A é uma álgebra afim e $I \subset A$ é um ideal radical tal que $A / I$ possui dimensão finita, então $A / I \cong \mathbb{k}^{n}$.

Demonstração. Como $A / I$ possui dimensão finita, pela Proposição 1.1.16, seu ideal de Jacobson j é nilpotente. Porém, $A / I$ é reduzida, pois $I$ é radical. Assim, $\mathfrak{j}=0$. Além disso, $A / I$ possui uma quantidade finita de ideais maximais, sejam eles $m_{1} / I, \ldots, m_{k} / I$. Pelo Teorema Chinês do Resto (Teorema 1.1.13),

$$
\frac{A / I}{\mathrm{j}}=\frac{A / I}{\left(m_{1} / I\right) \ldots\left(m_{k} / I\right)} \cong \frac{A / I}{m_{1} / I} \times \cdots \times \frac{A / I}{m_{k} / I} \cong A / m_{1} \times \cdots \times A / m_{k} \cong \mathbb{k}^{n} .
$$

Definição 1.1.18. Seja $A$ uma álgebra afim. A dimensão de Krull de $A$ é o tamanho $k$ da maior cadeia de ideais primos de $A$ da forma

$$
P_{0} \varsubsetneqq P_{1} \varsubsetneqq P_{2} \varsubsetneqq \cdots \varsubsetneqq P_{k} .
$$

Se existem cadeias arbitrariamente longas de ideais primos em $A$, então dizemos que a dimensão de Krull de $A$ é infinita.

Existem outras noções de dimensão de uma álgebra, mas utilizaremos a dimensão de Krull, pois esta é suficiente para nós. Um fato não tão trivial, porém esperado, é que a dimensão de Krull de $\mathbb{k}\left[x_{1}, \ldots, x_{n}\right]$ é $n$, veja Corollary 10.13 de [Eis95].

\section{2 Álgebras de Lie}

Lembre que $\mathbb{k}$ é um corpo algebricamente fechado de característica 0. Alguns resultados dados nesta e próxima seções valem mesmo quando $k$ não é algebricamente fechado ou não tem característica 0, porém precisaremos de tal suposição futuramente e por isso suporemos isto desde já. Todos os espaços vetoriais, transformações lineares e outras estruturas algébricas terão k como base. A principal referência para esta seção é [Hum78]. Para uma referência em português, veja [SM10].

Definição 1.2.1. Seja $\mathfrak{g}$ um espaço vetorial sobre $k$. Dizemos que $\mathfrak{g}$ é uma álgebra de Lie, se estiver munido de uma operação bilinear (chamado de comutador ou colchete de Lie)

$$
[,]: \mathfrak{g} \times \mathfrak{g} \rightarrow \mathfrak{g}
$$


satisfazendo as seguintes propriedades

(i) $[x, x]=0, \forall x \in \mathfrak{g}$,

(ii) $[x,[y, z]]+[z,[x, y]]+[y,[z, x]]=0, \forall x, y, z \in \mathfrak{g}$.

A primeira condição é chamada, em alguns casos, de alternatividade. Caso k tenha característica diferente de dois, a primeira propriedade acima é equivalente a dizer que o produto é anti-simétrico, ou seja, $[x, y]=-[y, x], \forall x, y \in \mathfrak{g}$. Como estamos supondo que o corpo $\mathbb{k}$ tem característica 0 , as propriedades são equivalentes. Além disso, a segunda condição é chamada de identidade de Jacobi e podemos escrevê-la alternativamente na forma

$$
[x,[y, z]]=[[x, y], z]+[y,[x, z]] .
$$

Naturalmente, dizemos que um subespaço $\mathfrak{a} \subset \mathfrak{g}$ é uma subálgebra de $\mathfrak{g}$, se $\mathfrak{a}$ é fechado pelo colchete, isto é, $[x, y] \in \mathfrak{a}$, se $x, y \in \mathfrak{a}$.

Exemplo 1.2.2. Para um espaço vetorial qualquer $\mathfrak{g}$, podemos definir o comutador $[x, y]=0$ para todo $x, y \in \mathfrak{g}$. Uma álgebra de Lie tal que o comutador é zero para todo elemento nela é chamada de álgebra de Lie abeliana. Todo subespaço de uma álgebra de Lie abeliana é uma álgebra de Lie abeliana. Além disso, todo subespaço de dimensão 1 de uma álgebra de Lie qualquer é uma álgebra de Lie abeliana.

Exemplo 1.2.3. Se $U$ é uma álgebra associativa, então podemos definir a álgebra de Lie associada a $U$, denotada por $\operatorname{Lie}(U)$, quando munido com o comutador

$$
[x, y]=x y-y x \forall x, y \in U .
$$

Exemplo 1.2.4. Seja $V$ um espaço vetorial, então o espaço vetorial $E n d(V)$ dos endomorfismos de $V$ é uma álgebra associativa quando munido com a composição de funções. Denotaremos a álgebra de Lie $\operatorname{End}(V)$ munida com o comutador definido no exemplo anterior por $\mathfrak{g l}(V)$, isto é, $\mathfrak{g l}(V)=\operatorname{Lie}(\operatorname{End}(V))$. Quando a dimensão de $V$ for finita, podemos identificar $\mathfrak{g l}(V)$ com o espaço das matrizes $n \times n, \mathfrak{g l}_{n}(\mathbb{k})$.

$\mathfrak{g l}_{n}(\mathbb{k})$ possui subálgebras muito importantes, aqui evidenciaremos apenas uma delas,

$$
\mathfrak{s l}_{n}(\mathbb{k})=\left\{x \in \mathfrak{g l}_{n}(\mathbb{k}): \operatorname{tr}(x)=0\right\},
$$

onde tr $: \mathfrak{g l}_{n}(\mathbb{k}) \rightarrow \mathfrak{g l}_{n}(\mathbb{k})$ é a transformação linear traço.

Notação 1.2.5. Sejam $V$ um espaço vetorial sobre $\mathbb{k}, A \subset \mathbb{k}$ e $B \subset V$, então denotaremos

$$
\operatorname{span}_{A} B=\left\{\sum_{i=1}^{l} a_{i} b_{i} \mid l \in \mathbb{Z}_{\geq 0}, a_{1}, \ldots, a_{l} \in A, b_{1}, \ldots, b_{l} \in B\right\} .
$$

Se $\mathfrak{g}$ é uma álgebra de Lie e $\mathfrak{a}, \mathfrak{b} \subset \mathfrak{g}$, então definimos

$$
[\mathfrak{a}, \mathfrak{b}]=\operatorname{span}_{\mathbb{k}}\{[a, b]: a \in \mathfrak{a}, b \in \mathfrak{b}\} .
$$

Claramente, temos que $\mathfrak{b}$ é uma subálgebra de $\mathfrak{a}$ se, e somente se, $[\mathfrak{b}, \mathfrak{b}] \subset \mathfrak{b}$. Além disso, chamamos $\mathfrak{b}$ de ideal de $\mathfrak{a}$ se $[\mathfrak{a}, \mathfrak{b}] \subset \mathfrak{b}$. Não é difícil mostrar que se $\mathfrak{g}$ é uma álgebra de Lie e $\mathfrak{a}, \mathfrak{b} \subset \mathfrak{g}$ são ideais de $\mathfrak{g}$, então $\mathfrak{a}+\mathfrak{b}, \mathfrak{a} \cap \mathfrak{b}$ e $[\mathfrak{a}, \mathfrak{b}]$ também são ideais de $\mathfrak{g}$.

Exemplo 1.2.6. Se $\mathfrak{g}$ é uma álgebra de Lie, então o conjunto $\mathfrak{g}^{\prime}=[\mathfrak{g}, \mathfrak{g}]$ é um ideal de $\mathfrak{g}$ e é chamado de álgebra derivada de $\mathfrak{g}$. De fato, segue da definição que $[\mathfrak{g}, \mathfrak{g}]$ é uma subálgebra de $\mathfrak{g}$. Além disso, como $[\mathfrak{g},[\mathfrak{g}, \mathfrak{g}]] \subset[\mathfrak{g}, \mathfrak{g}]$, temos que $\mathfrak{g}^{\prime}$ também é um ideal. 
O centro $Z(\mathfrak{g})=\{x \in \mathfrak{g}:[x, \mathfrak{g}]=0\}$ também é um ideal de $\mathfrak{g}$. Dado um subconjunto $X \in \mathfrak{g}$, podemos definir o centralizador de $X$ em $\mathfrak{g}$ definido por

$$
C_{\mathfrak{g}}(X)=\{x \in \mathfrak{g}:[x, X]=0\} .
$$

O centralizador de um subconjunto qualquer de $\mathfrak{g}$ é uma subálgebra de $\mathfrak{g}$ e, obviamente, $Z(\mathfrak{g})=$ $C_{\mathfrak{g}}(\mathfrak{g})$. Por fim, o normalizador de uma subálgebra $\mathfrak{a} \subset \mathfrak{g}$, definido como $N_{\mathfrak{g}}(\mathfrak{a})=\{x \in \mathfrak{g}:[x, \mathfrak{a}] \subset$ $\mathfrak{g}\}$, é a maior subálgebra de $\mathfrak{g}$ que contém $\mathfrak{a}$ como ideal.

Definindo

$$
\mathfrak{g}^{(0)}=\mathfrak{g} \quad \text { e } \quad \mathfrak{g}^{(i)}=\left[\mathfrak{g}^{(i-1)}, \mathfrak{g}^{(i-1)}\right], \text { para } i \geq 2
$$

temos uma sequência de ideais da álgebra de Lie $\mathfrak{g}$, chamada de série derivada. Além disso, podemos definir a série central descendente da álgebra de Lie $\mathfrak{g}$ como

$$
\mathfrak{g}^{1}=\mathfrak{g}, \quad \mathfrak{g}^{k}=\left[\mathfrak{g}, \mathfrak{g}^{k-1}\right] .
$$

Como o comutador de ideais é ideal, segue que $\mathfrak{g}^{i}$ é ideial de $\mathfrak{g}$ para todo $i \geq 1$. Esta série é chamada de descendente, pois $\mathfrak{g}^{i+1}=\left[\mathfrak{g}, \mathfrak{g}^{i}\right] \subset \mathfrak{g}^{i}, \log \mathrm{O}$

$$
\mathfrak{g}^{1} \supset \mathfrak{g}^{2} \supset \mathfrak{g}^{3} \supset \ldots
$$

É possível mostrar que $\mathfrak{g}^{i}$ é gerada por todos os possíveis colchetes entre $i$ elementos de $\mathfrak{g}$. Estas séries não foram criadas por um acaso, elas serão importantes na classificação de álgebras de Lie, portanto definiremos outros dois tipos de álgebras de Lie utilizando-as.

Definição 1.2.7. Uma álgebra de Lie $\mathfrak{g}$ é dita solúvel se existe $k$ tal que $\mathfrak{g}^{(k)}=\{0\}$. Por outro lado, dizemos que $\mathfrak{g}$ é nilpotente se existe $k$ tal que $\mathfrak{g}^{k}=\{0\}$.

Temos que $\mathfrak{g}$ é solúvel se, e somente se, $\mathfrak{g}^{\prime}$ é nilpotente. Além disso, como $\mathfrak{g}^{(i)} \subset \mathfrak{g}^{i}$, para todo $i \geq 1$, temos que toda álgebra nilpotente é solúvel. Como $\mathfrak{g}^{k}$ é o subespaço gerado por todos os possíveis colchetes entre $k$ elementos de $\mathfrak{g}$, temos que $\mathfrak{g}$ é solúvel se, e somente se, existe $k$ tal que $\left[x_{1},\left[x_{2},\left[x_{3},\left[\ldots,\left[x_{k}, y\right]\right] \ldots\right]=0\right.\right.$, para todo $x_{1}, \ldots, x_{k}, y \in \mathfrak{g}$. Por fim, toda subálgebra de uma álgebra de Lie solúvel (nilpotente) é solúvel (nilpotente) e, se $\mathfrak{a}, \mathfrak{b} \subset \mathfrak{g}$ são ideais solúveis da álgebra de Lie $\mathfrak{g}$, então $\mathfrak{a}+\mathfrak{b}$ também é solúvel. Há um teorema clássico da teoria de álgebras de Lie que utilizaremos durante este trabalho que garante a existência de um autovetor comum à todos os elementos de uma álgebra de Lie solúvel consistindo de endomorfismos de um espaço vetorial finita.

Teorema 1.2.8 (Teorema de Lie). Seja $V$ um espaço vetorial sobre $\mathbb{k}$ de dimensão finita e $L$ uma subálgebra solúvel de $\mathfrak{g l}(V)$. Se $V \neq 0$, então $V$ contém um autovetor comum a todos os endomorfismos em $L$.

Demonstração. Veja Theorem 4.1 de [Hum78].

Suponha que $\mathfrak{a} \subset \mathfrak{g}$ seja um ideal solúvel da álgebra de Lie $\mathfrak{g}$ que não está contido em nenhum outro ideal solúvel. Se $\mathfrak{b} \subset \mathfrak{g}$ é outro ideal solúvel de $\mathfrak{g}$, então $\mathfrak{a}+\mathfrak{b}$ também é solúvel e, pela maximalidade de $\mathfrak{a}, \mathfrak{b} \subset \mathfrak{a}$. Sendo assim, toda álgebra de Lie possui um único ideal solúvel que não está contido em nenhum outro ideal solúvel e este chamaremos de $\operatorname{Rad}(\mathfrak{g})$, o radical de $\mathfrak{g}$. Obviamente, $\mathfrak{g}$ é solúvel se, e somente se, $\mathfrak{g}=\operatorname{Rad}(\mathfrak{g})$. Quando $\mathfrak{g}$ é uma álgebra de Lie não nula tal que $\operatorname{Rad}(\mathfrak{g})=0$, chamaremos $\mathfrak{g}$ de semissimples.

Semelhante às outras estruturas algébricas, as álgebras de Lie possuem morfismos que as relacionam.

Definição 1.2.9. Sejam $\mathfrak{a}, \mathfrak{b}$ álgebras de Lie. Uma transformação linear $\varphi: \mathfrak{a} \rightarrow \mathfrak{b}$ é um homomorfismo de álgebras de Lie se $\varphi([x, y])=[\varphi(x), \varphi(y)]$. Se $\varphi$ for inversível, dizemos que $\varphi$ é isomorfismo. Dizemos que duas álgebras de Lie $\mathfrak{g}$ e $\mathfrak{h}$ são isomorfas, se existir um isomorfismo $\varphi: \mathfrak{g} \rightarrow \mathfrak{h}$. 
Como em outras estruturas algébricas, a imagem de um homomorfismo $\varphi: \mathfrak{a} \rightarrow \mathfrak{b}$ de álgebras de Lie é uma subálgebra de $\mathfrak{b}$. Além disso, o núcleo de $\varphi \operatorname{ker}(\varphi)=\{x \in \mathfrak{a}: \varphi(x)=0\}$ é um ideal de $\mathfrak{a}$ e $\varphi$ é injetora se, e somente se, $\operatorname{ker}(\varphi)=\{0\}$.

Exemplo 1.2.10. Seja $\mathfrak{g}$ uma álgebra de Lie. Para cada $x \in \mathfrak{g}$, considere a transformação linear $\operatorname{ad}_{x}: \mathfrak{g} \rightarrow \mathfrak{g}$ dada por $\operatorname{ad}_{x}(y)=[x, y]$. Utilizando a identidade de Jacobi, é possível mostrar que

$$
\operatorname{ad}_{[x, y]}(z)=\left(\operatorname{ad}_{x} \operatorname{ad}_{y}-\operatorname{ad}_{y} \operatorname{ad}_{x}\right)(z)=\left[\operatorname{ad}_{x}, \operatorname{ad}_{y}\right](z) \quad x, y, z \in \mathfrak{g} .
$$

Isto é, ad $: \mathfrak{g} \rightarrow \mathfrak{g l}(\mathfrak{g})$ é um morfismo de álgebras de Lie, chamado de representação adjunta, dado por

$$
\begin{aligned}
\text { ad }: \mathfrak{g} & \longrightarrow \mathfrak{g l}(\mathfrak{g}) \\
x \longmapsto \operatorname{ad}_{x}: \mathfrak{g} & \longrightarrow \mathfrak{g} \\
y & \longmapsto[x, y]
\end{aligned}
$$

Dado $x \in \mathfrak{g}$, se para algum $k \in \mathbb{Z}_{>\mathbb{0}}$ temos que $\left(\mathrm{ad}_{x}\right)^{k}=0$, então dizemos que $x$ é nilpotente. Se todos os elementos de $\mathfrak{g}$ são nilpotentes, então $\mathfrak{g}$ é chamada de localmente nilpotente. Se $\mathfrak{g}$ é nilpotente, então $\mathfrak{g}$ é localmente nilpotente. Pelo Teorema de Engel (veja [Hum78] Theorem 3.3), se $\mathfrak{g}$ é uma álgebra de Lie de dimensão finita que é localmente nilpotente, então $\mathfrak{g}$ é nilpotente. Dizemos que $x \in \mathfrak{g}$ é um elemento semissimples de $\mathfrak{g}$, se $a d_{x}$ é diagonalizável.

Utilizando a representação adjunta, podemos definir uma forma bilinear simétrica $\kappa: \mathfrak{g} \times \mathfrak{g} \rightarrow \mathbb{k}$ que possui um papel importante na classificação de álgebras de Lie semissimples, dada por

$$
\kappa(x, y)=\operatorname{tr}\left(a d_{x} a d_{y}\right) \quad x, y \in \mathfrak{g} .
$$

Esta é chamada de forma de Cartan-Killing e ela é associativa, no sentido de que

$$
\kappa([x, y], z)=\kappa(x,[y, z]) .
$$

Outra propriedade importante da forma de Cartan-Killing é que ela é invariante sobre autormorfismos de $\mathfrak{g}$, isto é, se $\phi \in A u t(\mathfrak{g})$, então

$$
\kappa(x, y)=\kappa(\phi(x), \phi(y)) .
$$

Como dito acima, a forma de Cartan-Killing possui uma papel importante na classificação de álgebras de Lie semissimples. Porém, ela também fornece um critério interessante para determinar se uma álgebra de Lie é solúvel.

Teorema 1.2.11 (Critério de Cartan). Seja $\mathfrak{g}$ uma álgebra de Lie de dimensão finita, então $\mathfrak{g}$ é solúvel se, e somente se, $\kappa(x, y)=0$ para todo $x \in \mathfrak{g}$ e para todo $y \in \mathfrak{g}^{\prime}$.

Demonstração. Veja Theorem 4.3 de [Hum78].

\subsubsection{Extensões Centrais de Álgebras de Lie}

Definição 1.2.12. Sejam $\mathfrak{a}, \mathfrak{b}$ e $\mathfrak{c}$ álgebras de Lie. Dizemos que

$$
0 \longrightarrow \mathfrak{a} \stackrel{\varphi}{\longrightarrow} \mathfrak{b} \stackrel{\psi}{\longrightarrow} \mathfrak{c} \longrightarrow 0,
$$

é uma sequência exata curta de álgebras de Lie, se $\varphi: \mathfrak{a} \rightarrow \mathfrak{b}$ e $\psi: \mathfrak{b} \rightarrow \mathfrak{c}$ são homomorfismos de álgebras de Lie tais que $\varphi$ é injetora, $\psi$ é sobrejetora e $\operatorname{ker}(\psi)=\operatorname{im}(\varphi)$. Se existir tal sequência exata curta de álgebras de Lie, dizemos que $\mathfrak{b}$ é uma extensão de $\mathfrak{c}$ por $\mathfrak{a}$.

Dadas álgebras de Lie $\mathfrak{g}$ e $\mathfrak{c}$, dizemos que uma extensão

$$
0 \longrightarrow \mathfrak{c} \stackrel{\varphi}{\longrightarrow} \mathfrak{e} \stackrel{\psi}{\longrightarrow} \mathfrak{g} \longrightarrow 0
$$


é trivial se $\mathfrak{e}=I \oplus \operatorname{ker}(\psi)$, onde $I$ é um ideal de $\mathfrak{e}$. Este tipo de extensão é sempre possível fazer, basta tomar $\mathfrak{e}$ como sendo a soma direta das álgebras de Lie $\mathfrak{c}$ e $\mathfrak{g}$. O desafio está em determinar extensões não triviais de uma álgebra de Lie, classificar tais extensões ou provar que $\mathfrak{g}$ pode ou não ser realizada como uma subálgebra da extensão $\mathfrak{e}$. Para isto, primeiro definiremos o que é um homomorfismo de extensões de álgebras de Lie.

Definição 1.2.13. Sejam $\mathfrak{a}$ e $\mathfrak{c}$ álgebras de Lie. Considere $\mathfrak{b}$ e $\mathfrak{b}^{\prime}$ duas extensões de $\mathfrak{c}$ por $\mathfrak{a}$. Dizemos que $\mathfrak{b}$ e $\mathfrak{b}^{\prime}$ são equivalentes se existir um isomorfismo de álgebras de Lie $f: \mathfrak{b} \rightarrow \mathfrak{b}^{\prime}$ tal que o diagrama

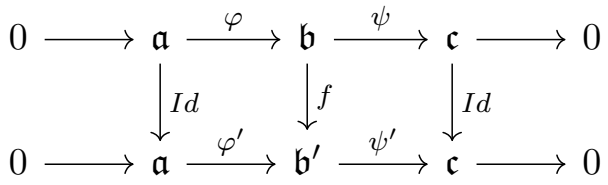

comuta.

A definição de extensões equivalentes define uma relação de equivalência entre as extensões de uma álgebra de Lie $\mathfrak{g}$ pela álgebra de Lie $\mathfrak{c}$. Para classificar as extensões de uma álgebras de Lie por outra, basta determinar as classes de equivalência de suas extensões.

Dadas álgebras de Lie $\mathfrak{g}$ e $\mathfrak{c}$, uma pergunta que podemos fazer é se $\mathfrak{g}$ pode ou não ser realizada como uma subálgebra de sua extensão e por $\mathfrak{c}$. Isto pode ser formalizado da seguinte forma: dizemos que a extensão de álgebras de Lie

$$
0 \longrightarrow \mathfrak{c} \stackrel{\varphi}{\longrightarrow} \mathfrak{e} \stackrel{\psi}{\longrightarrow} \mathfrak{g} \longrightarrow 0
$$

divide, se existe uma subálgebra $\mathfrak{s}$ de $\mathfrak{e}$ tal que $\mathfrak{e}=\mathfrak{s} \oplus$ ker $\psi$. Neste caso, $\mathfrak{s}$ não é necessariamente um ideal de $\mathfrak{e}$ e $\mathfrak{s} \cong \mathfrak{g}$.

Dizemos que a extensão $\mathfrak{e}$ de $\mathfrak{g}$ é central, se $\operatorname{ker}(\psi)$ está contido no centro $Z(\mathfrak{e})$ de $\mathfrak{e}$, onde $Z(\mathfrak{e})=\{x \in \mathfrak{e} \mid[x, \mathfrak{e}]=0\}$. Neste caso, como $\mathfrak{c} \cong \operatorname{im}(\varphi)=\operatorname{ker}(\psi)$, temos que $\mathfrak{c}$ é abeliana. O estudo das classes de equivalência das extensões centrais de uma álgebra de Lie está ligado ao estudo do grupo de cohomologia. Não trataremos deste assunto aqui, porém o leitor pode encontrar mais informações no capítulo VII do livro [HS97]. Dizemos que uma extensão central $\mathfrak{e}$ de $\mathfrak{g}$ é universal se para toda extensão central

$$
0 \longrightarrow \mathfrak{c} \stackrel{\varphi^{\prime}}{\longrightarrow} \mathfrak{e}^{\prime} \stackrel{\psi^{\prime}}{\longrightarrow} \mathfrak{g} \longrightarrow 0,
$$

existem únicos homomorfismos de álgebras de Lie $\Phi: \mathfrak{c} \rightarrow \mathfrak{c}^{\prime}$ e $\Psi: \mathfrak{e} \rightarrow \mathfrak{e}^{\prime}$ tais que o diagrama

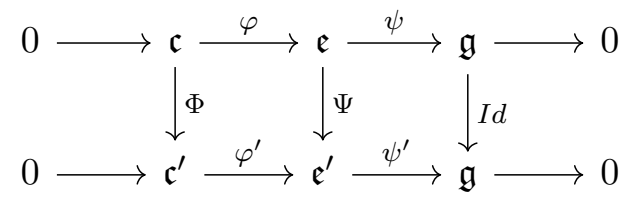

comuta.

Exemplo 1.2.14. Seja $\mathfrak{g}$ uma álgebra de Lie simples de dimensão finita, isto é, se $\mathfrak{g}$ é não abeliana e possui exatamente dois ideais. É possível mostrar que $\mathfrak{g}$ possui apenas extensões centrais triviais. Veja Proposition 6.3 do Capítulo VII do livro [HS97].

A teoria de extensões de álgebras de Lie e comohomologia de álgebras de Lie vai muito além do que mostramos aqui e possui resultados famosos, como os lemas de Whitehead e o Teorema de LeviMalcev (veja Capítulo VII de [HS97]). Nesta subseção, queríamos apenas mostrar a definição de extensão central universal de uma álgebra de Lie. Tocaremos neste assunto novamente no segundo capítulo deste trabalho. 


\subsubsection{Classificação das Álgebras de Lie Semissimples}

Lembre que $k$ é um corpo algebricamente fechado de característica 0.

Definição 1.2.15. Dizemos que uma álgebra de Lie é simples se $\mathfrak{g}$ não é abeliana e se possuir exatamente dois ideais distintos.

Temos que não existe uma álgebra de Lie simples de dimensão 1, a álgebra derivada de uma álgebra de Lie simples é ela mesma e seu centro é igual a 0 . Além de relacionar álgebras de Lie simples e semissimples, o seguinte teorema dá um critério útil para dizer se uma álgebra de Lie é semissimples.

Teorema 1.2.16. Seja $\mathfrak{g}$ uma álgebra de Lie de dimensão finita. São equivalentes:

(i) $\mathfrak{g}$ é semissimples.

(ii) A forma de Cartan-Killing é não degenerada.

(iii) $\mathfrak{g}$ se decompõe unicamente como soma direta finita de ideias simples. Isto é, existe $k \in \mathbb{Z}_{>0} e$ únicos ideais $\mathfrak{g}_{1}, \ldots, \mathfrak{g}_{k}$ de $\mathfrak{g}$ tais que

$$
\mathfrak{g}=\mathfrak{g}_{1} \oplus \cdots \oplus \mathfrak{g}_{k}
$$

Demonstração. A prova da equivalência entre o primeiro e segundo item está em Theorem 5.1 de [Hum78]. O primeiro item implica o terceiro pelo Theorem 5.2 de [Hum78]. E o terceiro implica o segundo, pois a forma de Cartan-Killing é não degenerada em cada um dos ideais $\mathfrak{g}_{i}$, então não será degenerada em $\mathfrak{g}=\mathfrak{g}_{1} \oplus \cdots \oplus \mathfrak{g}_{k}$.

A partir daqui, suporemos que $\mathfrak{g}$ é uma álgebra de Lie semissimples de dimensão finita. Além disso, lembre que $\mathfrak{g}$ é uma álgebra de Lie sobre o corpo $\mathbb{k}$ algebricamente fechado de característica 0. O objetivo é classificar todas as álgebras de Lie semissimples de dimensão finita sobre k. A estratégia se inicia com uma escolha de subálgebra de Cartan, a qual torna possível construir um sistema de raízes. Podemos associar cada sistema de raízes a um Diagrama de Dynkin, os quais estão todos determinados. Sendo assim, esperamos que os diagramas de Dynkin determinam as álgebras de Lie semissimples e vice-versa. Isto é verdade. Temos que é possível mostrar que escolhas diferentes da subálgebra de Cartan de uma álgebra de Lie semissimples determinam o mesmo diagrama de Dynkin. Isto é, podemos associar cada álgebra de Lie semissimples a um único diagrama de Dynkin. A volta também é verdadeira, cada diagrama de Dynkin pode ser associado a uma álgebra de Lie semissimples. Por fim, é possível mostrar que duas álgebras de Lie semissimples são isomorfas se e somente se possuem o mesmo diagrama de Dynkin. Além de definir o que são estes objetos, apontaremos os principais resultados e os passos que levam a construção do diagrama de Dynkin a partir da álgebra de Lie semissimples. Tudo isto pode ser visto com detalhes em [Hum78]. Caso o leitor queira uma referência em português, o livro [SM10] pode ser utilizado.

Proposição 1.2.17. Para cada $x \in \mathfrak{g}$, existem únicos $x_{n}, x_{s} \in \mathfrak{g}$ tais que $x=x_{s}+x_{n}, x_{s}$ é um elemento semissimples ( $\operatorname{ad}_{x_{s}}$ é diagonalizável), $x_{n}$ é um elemento nilpotente. Além disso, se $V$ é um espaço vetorial de dimensão finita, para cada representação $\rho: \mathfrak{g} \rightarrow \mathfrak{g l}(V)$, temos que $\rho\left(x_{s}\right)$ é diagonalizável, $\rho\left(x_{n}\right)$ é nilpotente e

$$
\rho(x)=\rho\left(x_{s}\right)+\rho\left(x_{n}\right) .
$$

Demonstração. Veja [Hum78] Theorem e Corollary 6.4.

Uma subálgebra de $\mathfrak{g}$ tal que todos seus elementos são semissimples é chamada de subálgebra toral. Uma subálgebra toral de $\mathfrak{g}$ é chamada de subálgebra maximal toral, se não existir outra subálgebra toral de $\mathfrak{g}$ contendo propriamente ela. Uma subálgebra de $\mathfrak{g}$ nilpotente cujo normalizador é ela mesma é chamada de subálgebra de Cartan. 
Teorema 1.2.18. Seja $\mathfrak{g}$ uma álgebra de Lie de dimensão finita semissimples sobre um corpo algebricamente fechado de característica 0 . Seja $\mathfrak{a}, \mathfrak{b} \subset \mathfrak{g}$ subálgebras de $\mathfrak{g}$, então

(i) Uma subálgebra toral é abeliana.

(ii) Existem subálgebras de Cartan de $\mathfrak{g}$.

(iii) a é uma subálgebra maximal toral se, e somente se, a é uma subálgebra de Cartan.

(iv) Se $\mathfrak{a}$ e $\mathfrak{b}$ são subálgebras de Cartan, então existe $\sigma \in A u t(\mathfrak{g})$ tal que $\mathfrak{a}=\sigma^{-1} \mathfrak{b} \sigma$. Isto é, todas as subálgebras de Cartan são conjugadas entre si e, portanto, isomorfas.

Demonstração. A prova para cada item deste teorema está no [Hum78], porém estão dividas em várias partes. A prova do primeiro item está na seção 8.1. As provas do segundo item e terceiro estão na Seção 15.3. Por fim, a prova do último item está na Seção 16.4.

Seja $\mathfrak{h} \subset \mathfrak{g}$ uma subálgebra de Cartan. Sendo assim, pelo teorema acima, hy é abeliana e, portanto, $\{\operatorname{ad}(x) \in \mathfrak{g l}(\mathfrak{g}): x \in \mathfrak{h}\}$ é uma família de operadores simultaneamente diagonalizáveis, pois são diagonalizáveis e comutam entre si. Isto é, a álgebra de Lie $\mathfrak{g}$ se decompõe como soma direta de subespaços da forma

$$
\mathfrak{g}_{\alpha}=\{x \in \mathfrak{g}:[h, x]=\alpha(h) x \text { para todo } x \in \mathfrak{h}\},
$$

onde $\alpha \in \mathfrak{h}^{*}$. Se $\alpha \neq 0$ e $\mathfrak{g}_{\alpha} \neq 0$, chamamos $\alpha$ de raiz. Os subespaços não nulos $\mathfrak{g}_{\alpha}$, com $\alpha \neq 0$, são chamados de espaços de raízes. O conjunto

$$
\Phi=\left\{\alpha \in \mathfrak{h}^{*}: \alpha \neq 0 \text { e } \mathfrak{g}_{\alpha} \neq 0\right\}
$$

é chamado de sistema de raízes.

Lema 1.2.19. Se $\mathfrak{h}$ é uma subálgebra de Cartan de $\mathfrak{g}$ e $\alpha, \beta \in \mathfrak{h}^{*}$, então $\left[\mathfrak{g}_{\alpha}, \mathfrak{g}_{\beta}\right] \subset \mathfrak{g}_{\alpha+\beta}$. Além disso, se $\alpha+\beta \neq 0, x \in \mathfrak{g}_{\alpha}$ e $y \in \mathfrak{g}_{\beta}$, ent $\tilde{a} o \kappa(x, y)=0$.

Além disso, se $\alpha \in \Phi$, então a dimensão de $\mathfrak{g}_{\alpha}$ é 1 . Temos ainda que se $x_{\alpha} \in \mathfrak{g}_{\alpha}$ é não nulo, então existe $y_{\alpha} \in \mathfrak{g}_{-\alpha}$ e $h_{\alpha}=\left[x_{\alpha}, y_{\alpha}\right] \in \mathfrak{h}$ tal que o homomorfismo de álgebras de Lie definido como $x_{\alpha} \mapsto\left[\begin{array}{ll}0 & 1 \\ 0 & 0\end{array}\right], y_{\alpha} \mapsto\left[\begin{array}{ll}0 & 0 \\ 1 & 0\end{array}\right]$ e $h_{\alpha} \mapsto\left[\begin{array}{cc}1 & 0 \\ 0 & -1\end{array}\right]$ é um isomorfismo.

Demonstração. Veja Proposition 8.1 e Proposition 8.4 de [Hum78]

Proposição 1.2.20. Seja $\mathfrak{h}$ uma subálgebra de Cartan de $\mathfrak{g}$, então $\mathfrak{h}=\mathfrak{g}_{0}$ e a forma de CartanKilling restrita a h é não degenerada.

Demonstração. Veja a seção 8.2 de [Hum78].

Pela Proposição 1.2.20, $\kappa$ é uma forma bilinear simétrica não degenerada quando restrita a $\mathfrak{h}$. Sendo assim, podemos identificar $\mathfrak{h}$ e $\mathfrak{h}^{*}$, utilizando que para cada $\alpha \in \mathfrak{h}^{*}$ existe um único $t_{\alpha} \in \mathfrak{h}$ tal que $\alpha(h)=\kappa\left(t_{\alpha}, h\right)$, para todo $\mathfrak{h} \in \mathfrak{h}$. Temos ainda que $\kappa$ induz uma forma bilinear simétrica não degenerada em $\mathfrak{h}^{*}$ dada por

$$
\langle\alpha, \beta\rangle=\kappa\left(t_{\alpha}, t_{\beta}\right) \quad \alpha, \beta \in \mathfrak{h}^{*} .
$$

Para cada $\alpha \in \Phi$, considere a transformação linear

$$
\sigma_{\alpha}(\beta)=\beta-2 \frac{\langle\beta, \alpha\rangle}{\langle\alpha, \alpha\rangle} \alpha, \quad \beta \in \Phi,
$$

então $\sigma_{\alpha}$ é um elemento de $\operatorname{End}(\mathfrak{h})$ inversível. Além disso, considere $\mathfrak{h}_{\mathbb{Q}}^{*}=\operatorname{span}_{\mathbb{Q}} \Phi$, então $h_{\mathbb{Q}}^{*}$ é um $\mathbb{Q}$-espaço vetorial. 
Proposição 1.2.21. Quando restrito a $\mathfrak{h}_{\mathbb{Q}}^{*},\langle\cdot, \cdot\rangle$ possui valores racionais e é uma forma bilinear simétrica positiva definida para $\mathfrak{h}_{\mathbb{Q}}^{*}$. Além disso, $\operatorname{dim}_{\mathbb{Q}} \mathfrak{h}_{\mathbb{Q}}^{*}=\operatorname{dim}_{\mathbb{Q}} \mathfrak{h}_{\mathbb{Q}}=\operatorname{dim}_{\mathbb{k}} \mathfrak{h}$. Temos ainda que:

(i) $\Phi$ é finito, gera $\mathfrak{h}_{\mathbb{Q}}^{*}$ e não contém 0 .

(ii) Se $\alpha \in \Phi$, então os únicos múltiplos de $\alpha$ em $\Phi$ são $\alpha e-\alpha$.

(iii) Se $\alpha \in \Phi$, então $\sigma_{\alpha}(\Phi)=\Phi$,

(iv) Se $\alpha, \beta \in \Phi$, então $2 \frac{\langle\beta, \alpha\rangle}{\langle\alpha, \alpha\rangle} \in \mathbb{Z}$.

Demonstração. Veja Theorem 8.5 de [Hum78].

Dizemos que $\Delta \subset \Phi$ é um sistema de raizes simples (ou base), se $\Delta$ é uma base do Q-espaço vetorial $\mathfrak{h}_{\mathbb{Q}}$ e, para cada $\beta \in \Phi \operatorname{com} \beta=\sum_{\alpha \in \Delta} a_{\alpha} \alpha$ temos que $\left\{a_{\alpha}: \alpha \in \Delta\right\}$ é um conjunto com números apenas não positivos ou apenas não negativos.

Teorema 1.2.22. Todo sistema de raizes possui um sistema de raizes simples.

Demonstração. Na subseção 10.1 de [Hum78], além de ser provado que todo sistema de raízies possui base, também é provado como todas as bases de $\Phi$ são obtidas.

Fixe um sistema de raízes simples $\Delta$ de $\Phi$. Chamamos os elementos $\alpha \in \Delta$ de raízes simples. Além disso, dizemos que $\beta \in \Phi$ é positiva, se os coeficientes de $\beta$ em relação a base $\Delta$, são positivos. Caso contrário, dizemos que é $\beta$ é negativa. Denote por $\Phi_{+}$o conjunto de todas as raízes positivas em $\Phi$ e $\Phi_{-}$o conjunto de todas as raízes negativas, então $\Phi_{-}=-\Phi_{+}$.

Isto nos permite definir a decomposição triangular de $\mathfrak{g}$ em relação a $\Delta$ dada por

$$
\mathfrak{g}=\mathfrak{g}_{+} \oplus \mathfrak{h} \oplus \mathfrak{g}_{-}, \text {onde } \mathfrak{g}_{+}=\bigoplus_{\alpha \in \Phi_{+}} \mathfrak{g}_{\alpha} \text { e } \mathfrak{g}_{-}=\bigoplus_{\alpha \in \Phi_{-}} \mathfrak{g}_{\alpha}
$$

Fixe uma ordem das raízes simples $\left\{\alpha_{1}, \ldots, \alpha_{l}\right\}$ de $\Phi$. A matriz $C=\left(c_{i j}\right)$ dada por

$$
c_{i j}=2 \frac{\left\langle\alpha_{j}, \alpha_{i}\right\rangle}{\left\langle\alpha_{i}, \alpha_{i}\right\rangle}
$$

é chamada de matriz de Cartan e os coeficientes $c_{i j}$ são chamados de inteiros de Cartan. A matriz de Cartan determina o sistema de raízes $\Phi$ completamente, via isomorfismo. Isso quer dizer que podemos reconstruir $\Phi$ utilizando a matriz de Cartan. Por outro lado, por causa das restrições de $\langle\cdot, \cdot\rangle$ em $\Phi$, temos que as possibilidades para os inteiros de Cartan são bem limitadas.

Lema 1.2.23. Seguindo a notação dada acima, temos que

(i) $C$ é uma matriz positiva definida,

(ii) $c_{i i}=2$ para todo $i$,

(iii) $c_{i j}=0,-1,-2$ ou -3 ,

(iv) $c_{j i}=-1$, se $c_{i j}=-2$ ou $c_{i j}=-3 e$

(v) $c_{i j}=0$ se, e somente se, $c_{j i}=0$.

Demonstração. Como $\langle\cdot, \cdot\rangle$ define uma forma simétrica positiva definida em $\mathfrak{h}^{*}$, temos que a matriz $\left(\left\langle\alpha_{i}, \alpha_{j}\right\rangle\right)_{i, j=1}^{l}$ é positiva definida e $\left\langle\alpha_{i}, \alpha_{i}\right\rangle>0$, para todo $i=1, \ldots, l$. Sendo assim, $C$ também é positiva definida. A prova dos outros itens do lema pode ser encontrada na Subsecção 6.5.1 de [SM10].

Nem toda matriz que satisfaz tais condições é uma matriz de Cartan de algum sistema simples de raízes. 
Definição 1.2.24. Seja $\left\{\alpha_{1}, \ldots, \alpha_{l}\right\}$ um sistema de raízes simples de $\Phi$. O diagrama de Dynkin relacionado a tais raízes simples é um grafo com $l$ vértices, representando cada uma das raízes simples, onde estes são ligados ou não por um, dois ou três arestas seguindo as regras:

(i) Se $c_{i j}=c_{j i}=0$, então não existe ligação entre os vértices $\alpha_{i}$ e $\alpha_{j}$ :

\section{$\dot{\alpha}_{i} \quad \dot{\alpha}_{j}$}

(ii) Se $c_{i j}=c_{j i}=-1$, então os vértices $\alpha_{i}$ e $\alpha_{j}$ são ligados por uma aresta:

\section{$\stackrel{\bullet}{\alpha_{i}} \dot{\alpha}_{j}$}

(iii) Se $c_{i j}=-2$ ou $c_{j i}=-2$, então os vértices $\alpha_{i}$ e $\alpha_{j}$ são ligados por duas arestas, que serão orientadas da seguinte forma: caso $c_{i j}=-2$,

$$
\stackrel{\bullet \bullet}{\alpha_{i} \alpha_{j}}
$$

caso $c_{j i}=-2$,

$$
\stackrel{\bullet \bullet}{\alpha_{i} \alpha_{j}}
$$

(iv) Se $c_{i j}=-3$ ou $c_{j i}=-3$, então os vértices $\alpha_{i}$ e $\alpha_{j}$ são ligados por três arestas e são orientadas como no caso anterior.

Dado um diagrama de Dynkin, é possível determinar os inteiros de Cartan. Dessa forma, como comentado anteriormente, podemos reconstruir o sistema de raízes utilizando um diagrama de Dynkin.

O sistema de raízes $\Phi$ é chamado de irredutivel, se não pode ser particionado em uma união de dois subconjuntos próprios $\Phi_{1}$ e $\Phi_{2}$ tais que para todo $\alpha \in \Phi_{1}$ e $\beta \in \Phi_{2}$ temos que $\langle\alpha, \beta\rangle=0$. Por outro lado, um diagrama de Dynkin é dito conexo se é um grafo conexo, isto é, sempre é possível traçar um "caminho" entre dois vértices. Sistemas de raízes irredutíveis definem diagramas de Dynkin conexos.

Teorema 1.2.25. Os diagramas de Dynkin conexos são

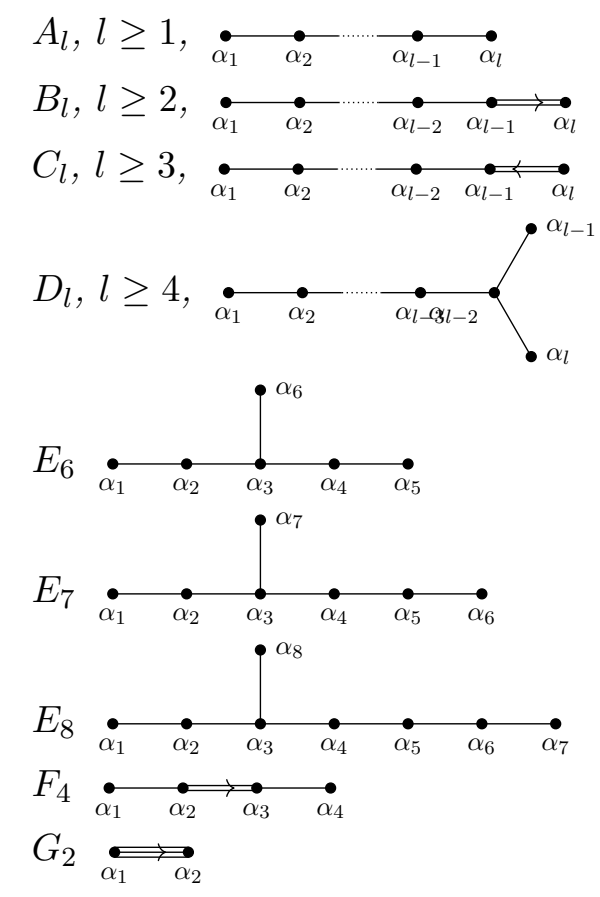


Demonstração. A prova detalhada deste teorema é dada na Subsseção 11.4 de [Hum78].

Concluímos esta seção com o seguinte teorema que concluí a classificação das álgebras de Lie semissimples. Não daremos uma construção explícita de cada álgebra de Lie simples representada no Teorema 1.2.25, porém essas construções podem ser encontradas na Seção 12 de [Hum78].

Teorema 1.2.26. (i) $S e \Delta_{1}$ e $\Delta_{2}$ são sistemas simples de raízes de uma subálgebra de Cartan de uma álgebra de Lie semissimples, então os diagramas de Dynkin associados a $\Delta_{1}$ e $\Delta_{2}$ são os mesmos. Portanto, $\mathfrak{h}$ determina um diagrama de Dynkin sem ambiguidade.

(ii) Se $\mathfrak{h}$ e $\mathfrak{h}^{\prime}$ são duas subálgebras de Cartan de uma álgebra de Lie semissimples, então os diagramas de Dynkin associados a $\mathfrak{h}$ e $\mathfrak{h}^{\prime}$ coincidem.

(iii) Duas álgebras de Lie semissimples isomorfas possuem os mesmos diagramas de Dynkin.

(iv) Cada um dos diagramas no Teorema 1.2.25 é o diagrama de Dynkin de uma álgebra de Lie semissimples.

(v) Duas álgebras de Lie semissimples com os mesmos diagramas de Dynkin são isormorfas.

Demonstração. O grupo de Weyl age transitivamente nas bases de um sistema de raízes (veja Theorem 10.3 de [Hum78]), então a matriz de Cartan é independente da escolha da base de um sistema de raízes e o item (i) está provado. Como todas as subálgebras de Cartan são conjugadas por autormorfismos de $\mathfrak{g}$ (veja Corollary 16.4 de [Hum78]), então o item (ii) está provado. A prova do item (iv) está na seção 12 de [Hum78]. Para a prova de (v), veja Theorem 14.2 de [Hum78]. Como cada álgebra de Lie semissimples determina um único diagrama de Dynkin, então todo isomorfismo entre duas álgebras de Lie semissimples induz um isomorfismo entre os diagramas de Dynkin. Este comentário e o item (v) implicam o item (iii).

\subsubsection{Conjuntos Parabólicos nos Sistemas de Raízes}

A definição de sistema de raízes pode ser dada para um espaço euclidiano. Seja $E$ um espaço vetorial de dimensão finita sobre $\mathbb{R}$ que está munido de uma aplicação bilinear não degenerada simétrica positiva definida $\langle\rangle:, E \times E \rightarrow E$. Para cada $\alpha \in E$, podemos definir o hiperplano gerado por $\alpha$ por $P_{\alpha}=\{\beta \in E \mid\langle\alpha, \beta\rangle=0\}$ e a reflexão em relação a $\alpha$ por

$$
\sigma_{\alpha}(\beta)=\beta-2 \frac{\langle\beta, \alpha\rangle}{\langle\alpha, \alpha\rangle} \alpha, \quad \beta \in E .
$$

Definição 1.2.27. Um subconjunto $R \subset E$ é chamado de sistema de raízes, se

(i) $R$ é finito, gera $E$ e não contém 0 .

(ii) Se $\alpha \in R$, então os únicos múltiplos de $\alpha$ em $R$ são $\alpha$ e $-\alpha$.

(iii) Se $\alpha \in R$, então $\sigma_{\alpha}(R)=R$,

(iv) Se $\alpha, \beta \in R$, então $2 \frac{\langle\beta, \alpha\rangle}{\langle\alpha, \alpha\rangle} \in \mathbb{Z}$.

Exemplo 1.2.28. A definição anterior é compatível com a definição de sistema de raízes dada na seção anterior. Se g é uma álgebra de Lie semissimples e hy é uma subálgebra de Cartan, então podemos definir $\Phi$ como feito na seção anterior. Pela Proposição 1.2.21, o espaço $\mathbb{R} \otimes_{\mathbb{Q}} \mathfrak{h}_{\mathbb{Q}}^{*}$ é um espaço euclidiano e $\left\{1 \otimes_{\mathbb{Q}} \alpha \mid \alpha \in \Phi \subset \mathfrak{h}_{\mathbb{Q}}\right\} \subset \mathbb{R} \otimes_{\mathbb{Q}} \mathfrak{h}_{\mathbb{Q}}^{*}$ satisfaz todas as condições da Definição 1.2.27. Na prática, ambas definições serão livremente utilizadas neste texto. A teoria de sistemas de raízes criada a partir da definição acima implica resultados importantes que podem ser aplicados na teoria de álgebras de Lie. Já vimos sua importância na classificação das álgebras de Lie semissimples, porém suas aplicações se estendem além disso. 
Os resultados clássicos da teoria de sistemas de raízes podem ser encontrados no terceiro capítulo de [Hum78]. Precisaremos de alguns deles que serão importantes na prova de alguns resultados deste trabalho. O primeiro resultado que precisaremos é o Lema 9.4 de [Hum78].

Lema 1.2.29. Sejam $\alpha, \beta \in \Phi$ tais que $\alpha \notin \mathbb{k} \beta$. Se $\langle\alpha, \beta\rangle>0$, então $\alpha-\beta$ é uma raiz. Se $\langle\alpha, \beta\rangle<0$, então $\alpha+\beta$ é uma raiz.

Como comentado após o Lema 9.4 de [Hum78], podemos considerar todas as raízes da forma $\alpha+i \beta$, onde $i \in \mathbb{Z}$. Sejam $r, q$ os maiores inteiros positivos tais que $\beta-r \alpha$ e $\beta+q \alpha$ são raízes, então $r-q=\frac{2\langle\beta, \alpha\rangle}{\langle\alpha, \alpha\rangle}$. Esta fórmula também é conhecida como fórmula de Killing.

Similarmente ao feito na seção anterior, dizemos que $B \subset R$ é um sistema de raízes simples (ou base), se $B$ é uma base do espaço vetorial $E$ e, para cada $\beta \in R \operatorname{com} \beta=\sum_{\alpha \in B} a_{\alpha} \alpha$, temos que $\left\{a_{\alpha}: \alpha \in B\right\}$ é um conjunto com números inteiros apenas não positivos ou apenas não negativos. Como comentado na seção anterior, é possível provar que todo sistema de raízes em $E$ possui uma base, para mais informações veja a subseção 10.1 de [Hum78]. Se $B$ é uma base do sistema de raízes $R$, então podemos definir o conjunto das raízes positivas de $R$ em relação a $B$ por

$$
R_{+}(B)=\left\{\beta \in R \mid \beta=\sum_{\alpha \in B} a_{\alpha} \alpha \text { e } a_{\alpha}>0 \text { para algum } \alpha \in B\right\} .
$$

Similarmente, podemos definir o conjunto de raízes negativas de $R$ em relação a $B$, denotado por $R_{-}(B)$ e vale que $R_{-}(B)=R_{+}(B)$.

Considere o grupo GL $(E)$ dos endomorfismos de $E$ inversíveis, então $\left\{\sigma_{\alpha} \mid \alpha \in R\right\} \subset \mathrm{GL}(E)$. O subgrupo $\mathcal{W}$ de $\mathrm{GL}(E)$ gerado por $\left\{\sigma_{\alpha} \mid \alpha \in R\right\}$ é chamado de grupo de Weyl associado à $R$. Pelo terceiro item da Proposição 1.2.21, $\mathcal{W}$ permuta os elementos de $R$. O grupo de Weyl desempenha um papel muito maior na teoria dos sistemas de raízes e na teoria de Lie, porém precisaremos de apenas do seguinte resultado.

Teorema 1.2.30. Sejam $R$ um sistema de raízes em $E$ e $B$ uma base de $R$. Se $\alpha \in B$, então $\sigma_{\alpha}$ permuta as raízes positivas diferentes de $\alpha$. Além disso,

1. $\sigma(B)$ é uma base de $R$ para todo $\sigma \in \mathcal{W}$.

2. Se $B^{\prime}$ é outra base de $R$, então existe $\sigma \in \mathcal{W}$ tal que $\sigma(B)=B^{\prime}$.

Demonstração. Veja as Subseções 10.2 e 10.3 de [Hum78].

As seguintes definições e resultados serão utilizados futuramente. Como não são resultados canônicos da teoria de álgebras de Lie semissimples e teoria dos sistemas de raízes, prová-los-emos. Para uma teoria mais detalhada destes resultados, veja Chapitre VI de [Bou72].

Definição 1.2.31. Seja $R$ um sistema de raízes em $E$ e seja $T \subset R$. Denotaremos $-T=\{-\alpha \mid$ $\alpha \in T\}$. Além disso, dizemos que

(i) $T$ é chamado de fechado se para todo $\alpha, \beta \in T$ tais que $\alpha+\beta \in R$, então $\alpha+\beta \in T$;

(ii) $T$ é chamado de parabólico, se $T$ é fechado e $T \cup(-T)=R$;

(iii) $T$ é chamado de simétrico, se $T=-T$.

Note que se $T \subset R$ é fechado, então $-T$ também é fechado. Além disso, se $T, S \subset R$ são fechados, então $T \cap S$ é fechado. Similarmente, podemos definir o que é um subconjunto fechado, parabólico e simétrico de um sistema de raízes $\Phi$ de uma álgebra de Lie semissimples $\mathfrak{g}$.

Proposição 1.2.32. Seja $R$ um sistema de raízes em $E$. Se $T \subset R$ é parabólico, então existe uma base $B$ de $R$ tal que $B \subset T$. 
Demonstração. Suponha que $T$ seja parabólico e seja $B$ a base tal que $R_{+}(B) \cap T$ tenha cardinalidade máxima, onde $R_{+}(B)$ denota as raízes positivas em relação a esta base. Se $B \subset T$, então $R_{+}(B) \subset T$, pois $T$ é fechado. Suponha que $B \backslash T \neq \emptyset$ e considere $\alpha \in B$ tal que $\alpha \notin T$. Como $T \cup-T=R$, segue que $-\alpha \in T$. Logo, $B^{\prime}=\sigma_{\alpha}(B)$ é uma base de $R$. Como $\sigma_{\alpha}$ permuta as raízes positivas $R_{+}(B)$ que não são $\alpha$ e $\sigma_{\alpha}(\alpha)=-\alpha$, segue que $-\alpha \in R_{+}\left(B^{\prime}\right)$, onde $R_{+}\left(B^{\prime}\right)$ denota as raízes positivas em relação à base $B^{\prime}$. Sendo assim, a cardinalidade de $R_{+}\left(B^{\prime}\right) \cap T$ é maior estritamente que a cardinalidade de $R_{+}(B) \cap T$. Contradição com o fato de que $R_{+}(B) \cap T$ tinha cardinalidade máxima.

Aplicando tal resultado na teoria de álgebras de Lie temos:

Corolário 1.2.33. Seja $\mathfrak{g}$ uma álgebra de Lie semissimples com subálgebra de Cartan $\mathfrak{h}$ e sistema de raizes $\Phi$. Seja $P \subset \Phi$ e defina

$$
\mathfrak{p}=\mathfrak{h} \oplus \sum_{\alpha \in P} \mathfrak{g}_{\alpha} .
$$

Se $P$ é parabólico, então $\mathfrak{p}$ é uma subálgebra de Lie de $\mathfrak{g}$ e existe uma escolha de raízes positivas em $\Phi_{+}$tal que a subálgebra de Borel $\mathfrak{b}_{+}=\mathfrak{h} \oplus \bigoplus_{\alpha \in \Phi_{+}} \mathfrak{g}_{\alpha} \subset \mathfrak{p}$.

Proposição 1.2.34. Sejam $R$ um sistema de raizes em $E$ e $T \subset R$. Se T é fechado e simétrico, então $T$ é um sistema de raízes para o espaço vetorial gerado por $T$.

Demonstração. $T$ é finito, gerador do espaço gerado por ele mesmo e não contém 0 . Se $\alpha \in T$, então $-\alpha \in T$, pois $T=-T$. Como $T \subset R$ e $R$ é um sistema de raízes, então os únicos múltiplos de $\alpha \in T$ em $T$ são $\alpha$ e $-\alpha$. Note que $2 \frac{\langle\beta, \alpha\rangle}{\langle\alpha, \alpha\rangle} \in \mathbb{Z}$, para todo $\alpha, \beta \in T$, pois $T \subset R$. Basta mostrar que $\beta-2 \frac{\langle\beta, \alpha\rangle}{\langle\alpha, \alpha\rangle} \in T$ para cada $\alpha, \beta \in T$. Suponha $\alpha, \beta \in T$ tal que $\beta \neq \alpha$ e $\beta \neq-\alpha$. Como $\alpha, \beta \in R$, existem inteiros não negativos $r$ e $q$ tais que

$$
\beta-r \alpha, \ldots, \beta-\alpha, \beta, \beta+\alpha, \ldots, \beta+q \alpha
$$

são raízes em $R$. Utilizando um argumento indutivo e a hipótese de que $T$ é simétrico e fechado, é fácil ver que cada uma destes elementos de $R$ são elementos de $T$. Logo, $T$ é um sistema de raízes para o espaço vetorial gerado por ele.

Corolário 1.2.35. Seja $\mathfrak{g}$ uma álgebra de Lie semissimples com subálgebra de Cartan $\mathfrak{h}$ e sistema de raízes $\Phi$. Se $T \subset \Phi$ é fechado e simétrico, então a subálgebra de Lie $\mathfrak{l}$ de $\mathfrak{g}$ gerada por $\left\{x_{\alpha}, y_{\alpha},\left[x_{\alpha}, y_{\alpha}\right] \mid \alpha \in T\right\}$ (veja Lema 1.2.19) é uma álgebra de Lie semissimples com subálgebra de Cartan $\mathfrak{h} \cap \mathfrak{l}=\operatorname{span}_{\mathbb{k}}\left\{\left[x_{\alpha}, y_{\alpha}\right] \mid \alpha \in T\right\}$ e sistema de raizes $T$.

\subsection{Representações de Álgebras de Lie}

A teoria das representações é o estudo de estruturas algébricas a partir de uma relação criada com as transformações lineares de espaços vetoriais. No caso de álgebras de Lie, isto nada mais é do que considerar homomorfismos de álgebras de Lie da forma $\mathfrak{g} \rightarrow \mathfrak{g l}(V)$, onde $\mathfrak{g}$ é uma álgebra de Lie qualquer e $V$ é um espaço vetorial. Quando $V$ possui dimensão finita $n$, já vimos que $\mathfrak{g l}(V)$ pode ser visto como o espaço das matrizes com coeficientes em $\mathbb{k}$, denotado por $\mathfrak{g l}_{n}(\mathbb{k})$.

Definição 1.3.1. Seja $\mathfrak{g}$ uma álgebra de Lie sobre $\mathbb{k}$ e $V$ uma k-espaço vetorial. Uma representação de $\mathfrak{g}$ em $V$ é um homomorfismos de álgebras de Lie $\rho: \mathfrak{g} \rightarrow \mathfrak{g l}(V)$. Isto é, $\rho$ é uma transformação linear e, para todo $v \in V, x, y \in \mathfrak{g}$, temos que

$$
\rho([x, y]) v=\rho(x) \rho(y) v-\rho(y) \rho(x) v
$$

A dimensão de $V$ pode ser qualquer, inclusive infinita. A dimensão da representação $\rho$ é, por definição, a dimensão de $V$. Já vimos um exemplo importante de uma representação que é a representação adjunta ad $: \mathfrak{g} \rightarrow \mathfrak{g l}(\mathfrak{g})$ (veja Exemplo 1.2.10). O estudo das representações de $\mathfrak{g}$ está intimamente ligado ao estudo de $\mathfrak{g}$-módulos. 
Definição 1.3.2. Seja $\mathfrak{g}$ uma álgebra de Lie e $V$ um k-espaço vetorial. Uma ação de $\mathfrak{g}$ em $V$ é uma aplicação bilinear

$$
\begin{aligned}
\mathfrak{g} \times V & \longrightarrow V \\
(x, v) & \longmapsto x \cdot v=x v
\end{aligned}
$$

satisfazendo

$$
[x, y] \cdot v=x \cdot(y \cdot v)-y \cdot(x \cdot v) \quad \text { para cada } x, y \in \mathfrak{g}, v \in V .
$$

Se existe um ação de $\mathfrak{g}$ em $V$, dizemos que $V$ é um $\mathfrak{g}$-módulo.

Toda ação de $\mathfrak{g}$ em $V$ define uma representação $\rho: \mathfrak{g} \rightarrow V$, dada por $\rho(x) v:=x \cdot v$. Por outro lado, toda representação $\rho: \mathfrak{g} \rightarrow V$ define uma ação de $\mathfrak{g}$ em $V$ dada por

$$
\begin{aligned}
\mathfrak{g} \times V & \longrightarrow V \\
(x, v) & \longmapsto x \cdot v:=\rho(x) v
\end{aligned}
$$

Desta forma, as duas estruturas são essencialmente iguais.

Um subespaço $W \subset V$ é um submódulo de $V$, se $W$ é um $\mathfrak{g}$-módulo. Todo $\mathfrak{g}$-módulo $V$ possui os submódulos triviais $V$ e $\{0\}$. Um $\mathfrak{g}$-módulo é dito simples (ou irredutivel), se possuir exatamente dois submódulos, os triviais. Neste sentido, não consideraremos o g-módulo nulo como irredutível. O termo irredutível é mais usado no estudo de representações e o termo simples é mais usado no estudo de módulos. Dizemos que $V$ é redutivel, se não for irredutível.

Exemplo 1.3.3. Lembre que $k$ é um corpo algebricamente fechado de característica 0. Se $\mathfrak{g}$ é uma álgebra de Lie solúvel ou abeliana, então qualquer representação irredutível de dimensão finita possui dimensão 1. Para provar isto, utilizaremos o Teorema de Lie (Teorema 1.2.8). Se $\rho: \mathfrak{g} \rightarrow \mathfrak{g l}(V)$ é uma representação da álgebra solúvel $\mathfrak{g}$, então $\rho(\mathfrak{g})$ é uma subálgebra solúvel de $\mathfrak{g l}(V)$. Pelo Teorema de Lie, existe um vetor $v \in V$ que é autovetor para todo elemento de $\rho(\mathfrak{g})$. Isto é, $k v$ é um submódulo de $V$. Logo, se $V$ é simples, $V \cong \mathbb{k} v$.

Definição 1.3.4. Sejam $V_{1}$ e $V_{2}$ g-módulos, diremos que uma transformação linear $\phi: V_{1} \rightarrow V_{2}$ é um homomorfismo de $\mathfrak{g}$-módulos de $V_{1}$ em $V_{2}$ se

$$
\phi(x v)=x \phi(v) \quad x \in \mathfrak{g}, v \in V_{1} .
$$

O conjunto de todos os homomorfismos de $V_{1}$ em $V_{2}$ será denotado por

$$
\operatorname{Hom}_{\mathfrak{g}}\left(V_{1}, V_{2}\right) \text {. }
$$

O seguinte lema, conhecido como Lema de Schur, é extremamente útil e possui diversas generalizações.

Lema 1.3.5 (Lema de Schur). Lembre que $\mathbb{k}$ é algebricamente fechado. Seja $\mathfrak{g}$ uma álgebra de Lie e sejam $V_{1}$ e $V_{2}$ duas representações irredutiveis de $\mathfrak{g}$.

(i) $S e \phi: V_{1} \rightarrow V_{2}$ é um homomorfismo de $\mathfrak{g}$-módulos, então $\phi$ é o homomorfismo nulo ou é um isomorfismo.

(ii) Se $V_{1}$ possui dimensão finita e $\phi: V_{1} \rightarrow V_{1}$ é um homomorfismo de $\mathfrak{g}$-módulos, então $\phi$ é um múltiplo da identidade.

(iii) Suponha que $\mathbb{k}$ seja não enumerável ou $\mathfrak{g}$ possua dimensão finita. Suponha que $V_{1}$ possua dimensão enumerável e $\phi: V_{1} \rightarrow V_{1}$ é um homomorfismo de $\mathfrak{g}$-módulos, então $\phi$ é um múltiplo da identidade. Ou seja, $\operatorname{Hom}_{\mathfrak{g}}(V, V) \cong \mathbb{k}$.

Demonstração. (i) O núcleo de $\phi$ é um submódulo de $V_{1}$. Como $V_{1}$ é simples, temos que $\phi$ é injetora ou $\phi$ é nula. Por outro lado, a imagem de $\phi$ é um submódulo de $V_{2}$. Se $\phi$ é injetora, como $V_{2}$ é simples, temos que $\phi$ é sobrejetora. Segue que $\phi$ é isomorfismo. 
(ii) Como $\mathbb{k}$ é algebricamente fechado, existe $\lambda \in \mathbb{k}$ e $v \in V_{1}$ tal que $\phi(v)=\lambda v$. Note que $\lambda$ Id $: V_{1} \rightarrow V_{1}$ é um homomorfismo de $\mathfrak{g}$-módulos. Logo, o núcleo do homomorfismo de $\mathfrak{g}$ módulos $\phi-\lambda$ Id é não nulo. Como $V_{1}$ é simples, temos que o núcleo de $\phi-\lambda$ Id é igual à $V_{1}$. Portanto, $\phi=\lambda I$.

(iii) Provaremos este item supondo que $\mathbb{k}$ não é enumerável, mas este item vale se supormos que $\mathfrak{g}$ possua dimensão finita e $\mathbb{k}$ é enumerável, veja [Qui69]. Como a composição e soma de elementos de $D=\operatorname{Hom}_{\mathfrak{g}}\left(V_{1}, V_{1}\right)$ são elementos de $D$, temos que $D$ é uma $\mathbb{k}$-álgebra associativa onde a aplicação identidade $I d$ é a unidade. Pelo primeiro item, todo elemento não nulo de $D$ é inversível. Ou seja, $D$ é um anel com divisão. Além disso, também pelo primeiro item, se $0 \neq v \in V_{1}$ e $\psi \in D$, então $\psi(v)=0$ se, e somente se, $\psi=0$. Logo, a transformação linear

$$
\begin{aligned}
& D \longrightarrow V_{1} \\
& \psi \mapsto \psi(v)
\end{aligned}
$$

é injetora, implicando que $D$ possui dimensão enumerável. Seja $\phi \in D$ tal que $\phi$ não é múltiplo da identidade, ou seja, $\phi-c I d \neq 0$ para todo $c \in \mathbb{k}$. Como $D$ um anel com divisão e $\phi$ não é nulo, segue que $\phi-c I d \neq 0$ é inversível para todo $c \in \mathbb{k}$. Para todo $f(x)=\sum_{i=0}^{n} a_{i} x^{i} \in \mathbb{k}[x]$, defina $f(\phi)=\sum_{i=0}^{n} a_{i} \phi^{i} \in D$. Como $\mathbb{k}$ é algebricamente fechado e $\phi-c I d$ é inversível para todo $c \in \mathbb{k}$, temos que $0 \neq f(\phi) \in D$ é inversível para todo $f \in \mathbb{k}[x]$ não nulo. Sendo assim, $(g(\phi))^{-1} f(\phi)=0$ para algum $g \in \mathbb{k}[x]$ não nulo se, e somente se, $f(\phi)=0$ se, e somente se, $f=0$. Logo,

$$
\mathbb{k}(\phi)=\left\{(g(\phi))^{-1} f(\phi) \mid f, g \in \mathbb{k}[x], \quad g \neq 0\right\} \subset D
$$

possui dimensão não enumerável, já que $\left\{(\phi-a)^{-1} \mid a \in \mathbb{k}\right\}$ é um subconjunto linearmente independente não enumerável, pois k é não enumerável. O que é uma contradição, já que $D$ possui dimensão enumerável. Logo, $\phi$ é um múltiplo da identidade para todo $\phi \in D$.

Observação 1.3.6. Na versão apresentada, o terceiro item do Lema 1.3.5 é dado a Dixmier.

Seja $\rho_{i}: \mathfrak{g} \rightarrow \mathfrak{g l}\left(V_{i}\right), i \in I$, uma família de representações de $\mathfrak{g}$. Podemos definir naturalmente uma representação de $\mathfrak{g}$ em $\bigoplus_{i \in I} V_{i}$ dada por

$$
x \cdot\left(\sum_{i \in I} v_{i}\right)=\sum_{i \in I} \rho_{i}(x) v_{i} .
$$

Esta representação é chamada de soma direta das representações $\rho_{i}, i \in I$ e denotada por $\bigoplus_{i \in I} \rho_{i}$.

Um módulo é dito completamente redutivel se é soma direta de representações irredutíveis. Equivalentemente, uma representação é completamente redutível, se todo g-submódulo $W$ de $V$ admite um $\mathfrak{g}$-submódulo complementar $W^{\prime}$, isto é, $V=W \oplus W^{\prime}$. Nem todo $\mathfrak{g}$-módulo redutível é completamente redutível. Utilizando a representação adjunta, temos que g é um g-módulo. Os $\mathfrak{g}$-submódulos de $\mathfrak{g}$ são os ideais, portanto toda álgebra de Lie simples é irredutível como $\mathfrak{g}$-módulo e toda álgebra semissimples é completamente irredutível como g-módulo.

Também podemos definir uma representação no produto tensorial finito de representações de $\mathfrak{g}$. Sejam $\rho_{i}: \mathfrak{g} \rightarrow \mathfrak{g l}\left(V_{i}\right), i=1, \ldots, k$, representações de $\mathfrak{g}$ e denote $V=V_{1} \otimes \cdots \otimes V_{k}$. Seja $\rho: \mathfrak{g} \rightarrow \mathfrak{g l}(V)$ a única transformação linear satisfazendo

$$
\rho(x)\left(v_{1} \otimes \cdots \otimes v_{k}\right)=\sum_{i=1}^{k} v_{1} \otimes \cdots \otimes \rho_{i}(x) v_{i} \otimes \cdots \otimes v_{k},
$$

para cada $x \in \mathfrak{g}, v_{i} \in V_{i}, i=1, \ldots, k$. A transformação linear $\rho$ é uma representação de $\mathfrak{g}$ em $V$ e é chamada de produto tensorial das representações $\rho_{1}, \ldots, \rho_{k}$, denotada por $\rho=\rho_{1} \otimes \cdots \otimes \rho_{k}$. 
Observação 1.3.7. Sejam $\mathfrak{g}_{1}$ e $\mathfrak{g}_{2}$ álgebras de Lie. Sejam $V_{1}$ e $V_{2}$ módulos sobre $\mathfrak{g}_{1}$ e $\mathfrak{g}_{2}$, respectivamente, então $V_{1} \otimes V_{2}$ é um módulo sobre a soma direta de álgebras de Lie $\mathfrak{g}_{1} \oplus \mathfrak{g}_{2}$, onde a ação é induzida por

$$
\left(x_{1}+x_{2}\right)\left(v_{1} \otimes v_{2}\right)=\left(x_{1} v_{1}\right) \otimes v_{2}+x_{1} \otimes\left(x_{2} v_{2}\right), \quad x_{1} \in \mathfrak{g}_{1}, x_{2} \in \mathfrak{g}_{2}, v_{1} \otimes v_{2} \in V_{1} \otimes V_{2} .
$$

Basta ver que se $v_{1} \otimes v_{2} \in V_{1} \otimes V_{2}, x_{1}, y_{1} \in \mathfrak{g}_{1}$ e $x_{2}, y_{2} \in \mathfrak{g}_{2}$, então

$$
\begin{aligned}
& \left(\left(x_{1}+x_{2}\right)\left(y_{1}+y_{2}\right)-\left(y_{1}+y_{2}\right)\left(x_{1}+x_{2}\right)\right)\left(v_{1} \otimes v_{2}\right) \\
= & x_{1} y_{1} v_{1} \otimes v_{2}+y_{1} v_{1} \otimes x_{2} v_{2}+x_{1} v_{1} \otimes y_{2} v_{2}+v_{1} \otimes x_{2} y_{2} v_{2} \\
& -y_{1} x_{1} v_{1} \otimes v_{2}+x_{1} v_{1} \otimes y_{2} v_{2}+y_{1} v_{1} \otimes x_{2} v_{2}+v_{1} \otimes y_{2} x_{2} v_{2} \\
= & \left(x_{1} y_{1} v_{1}-y_{1} x_{1} v_{1}\right) \otimes v_{2}+v_{1} \otimes\left(x_{2} y_{2}-y_{2} x_{2}\right) v_{2} \\
= & \left(\left[x_{1}, y_{1}\right] v_{1}\right) \otimes v_{2}+v_{1} \otimes\left(\left[x_{2}, y_{2}\right] v_{2}\right) \\
= & {\left[x_{1}+x_{2}, y_{1}+y_{2}\right] }
\end{aligned}
$$

\subsection{1 Álgebra Universal Envolvente}

Definição 1.3.8. Seja $\mathfrak{g}$ uma álgebra de Lie. Uma álgebra envolvente de $\mathfrak{g}$ é um par $(U, \varphi)$, onde $U$ é uma álgebra associativa com unidade e $\varphi: \mathfrak{g} \rightarrow \operatorname{Lie}(U)$ é um homomorfismo de álgebras de Lie, onde $\operatorname{Lie}(U)$ denota a estrutura de álgebra de Lie definida em $U$ por $[a, b]=a b-b a$.

Exemplo 1.3.9. Se $\rho: \mathfrak{g} \rightarrow \mathfrak{g l}(V)$ é uma representação de $\mathfrak{g}$, então $(E n d(V), \rho)$ é uma álgebra envolvente de $\mathfrak{g}$.

Definição 1.3.10. Uma álgebra envolvente universal de $\mathfrak{g}$ é uma álgebra envolvente $(U(\mathfrak{g}), \pi)$ tal que para toda álgebra envolvente de $\mathfrak{g}(U, \varphi)$ existe um único homomorfismo de álgebras associativas com unidade $\phi: U(\mathfrak{g}) \rightarrow U$ que faz o seguinte diagrama de homomorfismos de álgebras de Lie comutar

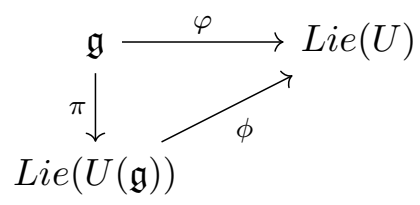

Proposição 1.3.11. Se $\mathfrak{g}$ possui uma álgebra envolvente universal, então essa é única a menos de isomorfismo.

Demonstração. Suponha que hajam duas álgebras envolventes universais, $(U(\mathfrak{g}), \pi)$ e $\left(U(\mathfrak{g})^{\prime}, \pi^{\prime}\right)$. Então existem $\phi^{\prime}: U(\mathfrak{g}) \rightarrow U(\mathfrak{g})^{\prime}$ e $\phi: U(\mathfrak{g})^{\prime} \rightarrow U(\mathfrak{g})$ únicos tais que o seguinte diagrama comuta:

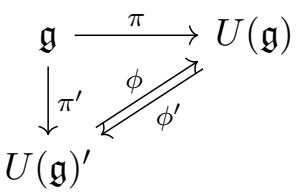

Daí $\phi \circ \phi^{\prime}: U(\mathfrak{g}) \rightarrow U(\mathfrak{g})$ e $\phi^{\prime} \circ \phi: U(\mathfrak{g}) \rightarrow U(\mathfrak{g})$ são homomorfismos de álgebras associativas com unidade, mas o seguinte diagrama comuta

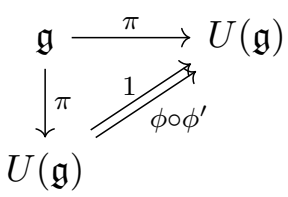

Como $U(\mathfrak{g})$ é um álgebra universal envolvente, pela unicidade de $\phi \circ \phi^{\prime}$, temos que $\phi \circ \phi^{\prime}=1$. Pelo mesmo argumento, podemos provar que $\phi^{\prime} \circ \phi=1$. Portanto, $U(\mathfrak{g})$ e $U(\mathfrak{g})^{\prime}$ são álgebras isomorfas. 
Seja $V$ um espaço vetorial sobre $\mathbb{k}$. Defina

$$
\begin{gathered}
T^{0}(V)=\mathbb{k} \\
T^{1}(V)=V \\
T^{2}(V)=V \otimes V \\
T^{m}(V)=V \otimes \cdots \otimes V(m \text { vezes })
\end{gathered}
$$

Sendo assim, podemos definir a álgebra tensorial de $V$ como sendo

$$
\mathcal{T}(V)=\bigoplus_{i \in \mathbb{Z}_{\geq 0}} T^{i}(V)
$$

onde a multiplicação é definida por concatenação, isto é,

$$
\left(x_{1} \otimes \cdots \otimes x_{i}\right)\left(y_{1} \otimes \cdots \otimes y_{j}\right)=x_{1} \otimes \cdots \otimes x_{i} \otimes y_{1} \otimes \ldots y_{j} .
$$

Desta forma, $\mathcal{T}(V)$ é uma álgebra associativa com unidade $1 \in \mathbb{k}=T^{0}$.

Se a dimensão de $V$ for $n$, então a álgebra tensorial $\mathcal{T}(V)$ é a álgebra universal associativa em $n$ geradores. A proposição a seguir diz isso em outras palavras.

Proposição 1.3.12. Seja $V$ um espaço vetorial de dimensão finita e $U$ uma álgebra associativa com unidade. Para toda transformação linear $\phi: V \rightarrow U$, existe um único homomorfismo de álgebras $\varphi: \mathcal{T}(V) \rightarrow U$ tal que $\varphi\left(1_{\mathcal{T}(V)}\right)=1_{U}$ e torna comutativo o diagrama

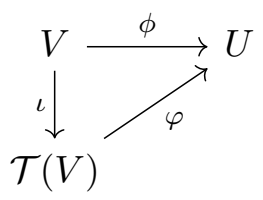

onde $\iota: V \rightarrow \mathcal{T}(V)$ é a inclusão de $V$ em $\mathcal{T}(V)$ que leva $V$ em $T^{1}(V)$.

Demonstração. Primeiramente, note que $\varphi(x \otimes y)=\varphi(x \cdot y)=\varphi(x) \varphi(y)$. Como $\varphi(v)=\phi(v)$ para todo $v \in V$ e $\phi$ é linear, então a unicidade de $\varphi$ segue do fato dele estar completamente definido por seu valor em $T^{1}(V)$.

Dado $\phi: V \rightarrow U$, defina $\varphi: \mathcal{T}(V) \rightarrow U$ para cada $x_{1} \otimes \cdots \otimes x_{i} \in T^{i}(V), y_{1} \otimes \cdots \otimes y_{j} \in T^{j}(V)$, $\lambda \in \mathbb{k}$, como

$$
\begin{gathered}
\varphi\left(x_{1} \otimes \cdots \otimes x_{i}\right)=\phi\left(x_{1}\right) \ldots \phi\left(x_{i}\right) \\
\varphi\left(x_{1} \otimes \cdots \otimes x_{i}+y_{1} \otimes \cdots \otimes y_{j}\right)=\phi\left(x_{1} \otimes \cdots \otimes x_{i}\right)+\phi\left(y_{1} \otimes \cdots \otimes y_{j}\right) \\
\varphi(\lambda)=\lambda
\end{gathered}
$$

Sendo assim, utilizando a multilinearidade do produto tensorial e que $\phi$ é linear, temos que $\varphi$ é um homomorfismo de álgebras.

Portanto, se $V$ tem dimensão finita igual a $n$ e $\left\{e_{1}, \ldots, e_{n}\right\}$ é base de $V$, então $T^{k}(V)$ tem dimensão $k^{n} \mathrm{e}$

$$
\left\{e_{i_{1}} \otimes \cdots \otimes e_{i_{k}}: i_{1}, \ldots, i_{k} \in\{1, \ldots, n\}\right\}
$$

é uma base de $T^{k}(V)$. Sendo assim, $\mathcal{T}(V)$ é isomorfo à álgebra de polinômios não comutativa nas variáveis $x_{1}, \ldots, x_{n}$.

Proposição 1.3.13. Toda álgebra de Lie $\mathfrak{g}$ possui uma álgebra envolvente universal.

Demonstração. Seja $\mathcal{T}(\mathfrak{g})$ a álgebra tensorial de $\mathfrak{g}, \iota: \mathfrak{g} \rightarrow \mathcal{T}(\mathfrak{g})$ a inclusão de $\mathfrak{g}$ em $\mathcal{T}(\mathfrak{g})$ e $I$ o ideal bilateral de $\mathcal{T}(\mathfrak{g})$ gerado por todos os elementos da forma

$$
x \otimes y-y \otimes x-[x, y] \quad x, y \in \mathfrak{g} .
$$


Seja $U(\mathfrak{g}):=\mathcal{T}(\mathfrak{g}) / I$ e a inclusão de $\mathfrak{g}$ em $U(\mathfrak{g})$ dada por

$$
\begin{aligned}
\pi: \mathfrak{g} & \rightarrow \operatorname{Lie}(U(\mathfrak{g})) \\
x & \longmapsto x+I
\end{aligned}
$$

que é linear. Para todo $x, y \in \mathfrak{g}$, temos que

$$
\pi([x, y])=[x, y]+I=x \otimes y-y \otimes x+I,
$$

então $\pi$ é um homomorfismo de álgebras de Lie.

Seja $A$ uma álgebra associativa com unidade e $\varphi: \mathfrak{g} \rightarrow \operatorname{Lie}(A)$ um homomorfismo de álgebras de Lie. Pela propriedade universal de $\mathcal{T}(\mathfrak{g})$, existe um homomorfismo de álgebras associativas com unidade $\phi: \mathcal{T}(\mathfrak{g}) \rightarrow A$ tal que $\phi \circ \iota=\varphi$. Para todo $x, y \in \mathfrak{g}$, temos que

$$
\begin{aligned}
\phi(x \otimes y-y \otimes x-[x, y]) & =\phi(x) \phi(y)-\phi(y) \phi(x)-\phi([x, y]) \\
& =\varphi(x) \varphi(y)-\varphi(y) \varphi(x)-\varphi([x, y])=0,
\end{aligned}
$$

pois $\varphi$ é um homomorfismo de álgebras de Lie. Logo, $I \subset$ ker $\phi$ e, portanto, existe um homomorfismo de álgebras associativas com unidade $\Phi: U(\mathfrak{g}) \rightarrow A$ tal que $\Phi \circ \pi(x)=\Phi(x+I)=\phi(x)$, para todo $x \in \mathfrak{g}$. Sendo assim, $(U(\mathfrak{g}), \pi)$ é uma álgebra envolvente de $\mathfrak{g}$.

Seja $\Phi^{\prime}: U(\mathfrak{g}) \rightarrow A$ outro homomorfismo de álgebras associativas com unidade tal que $\Phi^{\prime} \circ \pi(x)=$ $\Phi^{\prime}(x+I)=\varphi(x)$, para todo $x \in \mathfrak{g}$. Então, para todo $x_{1}, \cdots, x_{l} \in \mathfrak{g}$

$$
\begin{aligned}
\Phi^{\prime}\left(x_{1} \otimes \cdots \otimes x_{k}+I\right) & =\Phi^{\prime}\left(x_{1}+I\right) \cdots \Phi^{\prime}\left(x_{k}+I\right) \\
& =\varphi\left(x_{1}\right) \cdots \varphi\left(x_{k}\right) \\
& =\Phi\left(x_{1}+I\right) \cdots \Phi\left(x_{k}+I\right) \\
& =\Phi\left(x_{1} \otimes \cdots \otimes x_{k}+I\right) .
\end{aligned}
$$

Sendo assim, $\Phi=\Phi^{\prime}$. Logo, $(U(\mathfrak{g}), \pi)$ é uma álgebra envolvente universal de $\mathfrak{g}$.

Vendo a demonstração da Proposição 1.3.13, não é difícil chegar à conclusão de que $U(\mathfrak{g})$ é gerada por monômios da forma $x_{i_{1}} \ldots x_{i_{k}}=x_{i_{1}} \otimes \cdots \otimes x_{i_{k}}$, onde $\left\{x_{i}: i \in J\right\}$ é uma base de $\mathfrak{g} \mathrm{e}$ $i_{1}, \ldots, i_{j} \in J$. Supondo $J$ ordenado, podemos até utilizar as relações do comutador na base para ver que $U(\mathfrak{g})$ é gerada por $x_{i_{1}} \otimes \cdots \otimes x_{i_{k}}, i_{1} \leq \cdots \leq i_{k}$. O teorema a seguir afirma que tais monômios, na verdade, formam uma base de $U(\mathfrak{g})$.

Teorema 1.3.14 (Poincaré-Birkhoff-Witt). Seja $\mathfrak{g}$ uma álgebra de Lie e $\left\{x_{i}: i \in J\right\}$ uma base de $\mathfrak{g}$, onde $J$ é um conjunto de indices ordenado. Seja $\pi: \mathcal{T}(\mathfrak{g}) \rightarrow U(\mathfrak{g})$ a projeção canônica, então $\left.\pi\right|_{\mathfrak{g}}$ é injetora e

$$
\left\{\pi\left(x_{i_{1}}\right) \ldots \pi\left(x_{i_{k}}\right): i_{1}, \ldots, i_{k} \in J, i_{1} \leq \cdots \leq i_{k}\right\}
$$

é uma base de $U(\mathfrak{g})$

Demonstração. Veja a Seção 17.3 de [Hum78].

O Teorema de Poincaré-Birkhoff-Witt (PBW) possui consequências interessantes.

Corolário 1.3.15. Seja $\left\{x_{i}: i \in J\right\} \subset \mathfrak{g}$ um conjunto linearmente independente em $\mathfrak{g}$, então $\left\{\pi\left(x_{i}\right): i \in J\right\}$ é um conjunto linearmente independente de $U(\mathfrak{g})$.

A partir de agora, vamos escrever $x_{i_{1}} \ldots x_{i_{k}}$ no lugar de $\pi\left(x_{i_{1}}\right) \ldots \pi\left(x_{i_{k}}\right)$.

Precisaremos de uma outra versão do Teorema PBW neste trabalho. Para cada espaço vetorial $V$, defina a álgebra simétrica $S(V)=\mathcal{T}(V) / P$, onde $P$ é o ideal de $\mathcal{T}(V)$ gerado por todos os elementos da forma $x \otimes y-y \otimes x, x, y \in V$. 
Para isso, note que $U(\mathfrak{g})$ herda uma filtração de $\mathcal{T}(\mathfrak{g})$, dada por $U_{m}=\pi\left(T_{m}(\mathfrak{g})\right)$, onde $T_{m}(\mathfrak{g})=$ $\left.T^{0}(\mathfrak{g}) \oplus T^{1}(\mathfrak{g}) \oplus \cdots \oplus T^{m}(\mathfrak{g})\right)$, e podemos assim tomar a álgebra graduada associada

$$
\bar{U}(\mathfrak{g}):=\operatorname{gr}(U(\mathfrak{g}))=\bigoplus_{i=0} U_{m} / U_{m-1}
$$

onde $U_{-1}=\{0\}$. Podemos definir uma transformação linear $\sigma_{m}: T_{m}(\mathfrak{g}) \rightarrow U_{m} / U_{m-1}$ dada por $\sigma_{m}(w)=\pi(w)+U_{m-1}$. A partir de $\sigma_{i}, i \in \mathbb{Z}_{\geq 0}$, definimos um homomorfismo de álgebras $\sigma: \mathcal{T}(\mathfrak{g}) \rightarrow \bar{U}(\mathfrak{g})$, com $\sigma(1)=1$ e $\left.\sigma\right|_{T_{m}}=\sigma_{m}$. Para todo $x, y \in \mathfrak{g}$, note que

$$
\sigma(x \otimes y-y \otimes x)=\sigma_{2}(x \otimes y-y \otimes x)=\pi(x \otimes y-y \otimes x)+U_{1}=\pi([x, y])+U_{1}=0 .
$$

Logo, o ideal de $\mathcal{T}(\mathfrak{g})$ gerado por todos os elementos $x \otimes y-y \otimes x$ está contido no núcleo de $\sigma$ e, portanto, $\sigma$ induz um homomorfismo de álgebras $\tau: S(\mathfrak{g}) \rightarrow \bar{U}(\mathfrak{g})$.

Pelo Teorema de Poincaré-Birkhoff-Witt, temos imediatamente o seguinte resultado.

Corolário 1.3.16. O homomorfismo de álgebras $\tau: S(\mathfrak{g}) \rightarrow \bar{U}(\mathfrak{g})$ construído acima é um isomorfismo de álgebras de Lie.

Suponha que $\mathfrak{g}$ possua dimensão finita. A ação adjunta

$$
x \cdot x_{1} \ldots x_{l}=\sum_{i=1}^{l} x_{1} \cdots\left[x, x_{i}\right] \cdots x_{l}, \quad x, x_{1}, \ldots, x_{l} \in \mathfrak{a},
$$

torna $S(\mathfrak{g})$ um $\mathfrak{g}$-módulo. Da mesma forma, podemos considerar $U(\mathfrak{g})$ e $\bar{U}(\mathfrak{g})$ como sendo $\mathfrak{g}$-módulos. O homomorfismo de álgebras $\tau$ construído acima é obviamente um isomorfismo de $\mathfrak{g}$-módulos entre $S(\mathfrak{g})$ e $\bar{U}(\mathfrak{g})$.

Um elemento $u \in U(\mathfrak{g})$ é dito elemento simétrico de grau $m$, se existe $f \in T^{m}(\mathfrak{g})$ tal que $\pi(f)=u$. Seja $U^{m} \subset U(\mathfrak{g})$ o conjuntos dos elementos simétricos de grau $m$ em $U(\mathfrak{g})$, então $U_{m}=U_{m-1} \oplus U^{m}$ e existe um isomorfismo linear entre $U_{m}$ e $T^{m}(\mathfrak{g})$. Além disso, pelo Teorema de Poincaré-Birkhoff-Witt, $U(\mathfrak{g})=\bigoplus_{m=0}^{\infty} U^{m}$ como espaço vetorial.

Seja $S^{m}(\mathfrak{g})=\left\{x_{1} \otimes \cdots \otimes x_{m}+P \mid x_{1}, \ldots, x_{m} \in \mathfrak{g}\right\}$, então, por construção, o óbvio diagrama de transformações lineares bijetoras comuta (veja Lemme 2.4.2 de [Dix96])

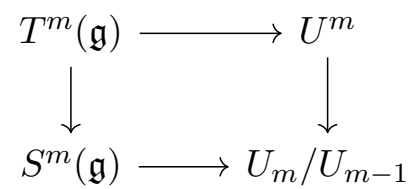

Em particular, para cada $m \geq 0$, o diagrama acima induz uma transformação linear bijetora $\omega_{m}: S^{m}(\mathfrak{g}) \rightarrow U^{m}$. A soma direta de tais transformações lineares induz uma transformação linear bijetora $\omega: S(\mathfrak{g}) \rightarrow U(\mathfrak{g})$. A transformação linear $\omega$ é chamada de aplicação simétrica e, explicitamente,

$$
\omega\left(x_{1} \cdots x_{l}\right)=\frac{1}{l !} \sum_{p \in \mathfrak{S}_{l}} x_{p(1)} \cdots x_{p(l)},
$$

para todo $x_{1}, \ldots, x_{l} \in \mathfrak{g}$, onde o produto no argumento de $\tau$ é feito em $S(\mathfrak{g})$ e o produto da somatória no lado direito da equação é feito em $U(\mathfrak{g})$.

Uma derivação $D$ de uma álgebra de Lie é uma transformação linear $D: \mathfrak{g} \rightarrow \mathfrak{g}$ tal que

$$
D([x, y])=[D(x), y]+[x, D(y)], \text { para todo } x, y \in \mathfrak{g} .
$$

Além disso, se $U$ é uma álgebra associativa com unidade, uma derivação $D: U \rightarrow U$ é uma transformação linear tal que

$$
D(x y)=D(x) y+x D(y), \text { para todo } x, y \in U .
$$


Toda derivação $D: \mathfrak{g} \rightarrow \mathfrak{g}$ estende unicamente para uma derivação $D^{\prime}: U(\mathfrak{g}) \rightarrow U(\mathfrak{g})$ e uma derivação $D^{\prime \prime}: S(\mathfrak{g}) \rightarrow S(\mathfrak{g})$. Além disso, $D^{\prime} \circ \tau=\tau \circ D^{\prime \prime}$ e, se existe $x \in \mathfrak{g}$ tal que $D=\mathrm{ad}_{x}$, temos que $D^{\prime}(u)=x u-u x$, para todo $u \in U(\mathfrak{g})$ (veja Proposition 2.4.9 de [Dix96]).

Proposição 1.3.17. O isomorfismo de espaços vetoriais $\omega: S(\mathfrak{g}) \rightarrow U(\mathfrak{g})$ construído acima é um isomorfismo de $\mathfrak{g}$-módulos.

Demonstração. Veja [Dix96, Proposition 2.4.10].

Podemos definir $I(\mathfrak{g}) \subset S(\mathfrak{g})$ como sendo o conjunto dos elementos $\mathfrak{g}$-invariantes, isto é,

$$
I(\mathfrak{g})=\{f \in S(\mathfrak{a}) \mid x \cdot f=0 \text { para todo } x \in \mathfrak{g}\} .
$$

Pela linearidade da representação, $I(\mathfrak{g})$ é claramente um subespaço vetorial de $S(\mathfrak{g})$, mas como $x \cdot f g=(x \cdot f) g+f(x \cdot f)$, para todo $x \in \mathfrak{g}$ e $f, g \in S(\mathfrak{g})$, temos que $I(\mathfrak{g})$ é uma subálgebra de $S(\mathfrak{g})$.

Corolário 1.3.18 ( [Dix96, Corollaire 2.4.11]). Seja $Z(\mathfrak{g}) \subset U(\mathfrak{g})$ o centro da álgebra $U(\mathfrak{g})$, então $\omega(I(\mathfrak{g}))=Z(\mathfrak{g})$.

Demonstração. Para cada $x \in \mathfrak{g}$, considere $D_{x}=\operatorname{ad}_{x}$. Seguindo a notação acima, $D_{x}^{\prime} \circ \tau=\tau \circ D_{x}^{\prime \prime}$, para todo $x \in \mathfrak{g}$. Como $\tau$ é isomorfismo, $\tau \circ D_{x}^{\prime \prime}(f)=0$ para todo $x \in \mathfrak{g}$ se, e somente se, $f \in I(\mathfrak{g})$. Por outro lado, $D_{x}^{\prime} \circ \tau(f)=0$ para todo $x \in \mathfrak{g}$ se, e somente se, $\tau(f) \in Z(\mathfrak{g})$. Logo, $f \in I(\mathfrak{g})$ se, e somente se, $\tau(f) \in Z(\mathfrak{g})$.

Corolário 1.3.19. Sejam $\mathfrak{g}_{1}, \ldots, \mathfrak{g}_{k}$ álgebras de Lie e $\mathfrak{g}=\bigoplus_{i=1}^{k} \mathfrak{g}_{k}$ como álgebra de Lie, então

$$
U(\mathfrak{g})=\bigotimes_{i=1}^{k} U\left(\mathfrak{g}_{k}\right) .
$$

Demonstração. Vamos provar para o caso $k=2$, para $k>2$ a prova é similar. Sejam $(U(\mathfrak{g}), \pi)$ a álgebra envolvente universal de $\mathfrak{g},\left(U\left(\mathfrak{g}_{1}\right), \pi_{1}\right)$ a álgebra envolvente universal de $\mathfrak{g}_{1}$ e $\left(U\left(\mathfrak{g}_{2}\right), \pi_{2}\right)$ a álgebra envolvente universal de $\mathfrak{g}_{2}$. Além disso, sejam $P_{1}: \mathfrak{g}_{1} \rightarrow \mathfrak{g}$ e $P_{2}: \mathfrak{g}_{2} \rightarrow \mathfrak{g}$ as projeções canônicas de $\mathfrak{g}_{1}$ e $\mathfrak{g}_{2}$ em $\mathfrak{g}$, respectivamente. Isto é, $P_{1}\left(x_{1}\right)=\left(x_{1}, 0\right)$ e $P_{2}\left(x_{2}\right)=\left(0, x_{2}\right)$, para todo $x_{1} \in \mathfrak{g}_{1}$ e $x_{2} \in \mathfrak{g}_{2}$.

Temos que $\pi \circ P_{1}: \mathfrak{g}_{1} \rightarrow U(\mathfrak{g})$ e $\pi \circ P_{1}: \mathfrak{g}_{2} \rightarrow U(\mathfrak{g})$ são homomorfismos de álgebras de Lie e, pela propriedade universal de $U(\mathfrak{g})$, existem homomorfismos de álgebras $\phi_{1}: U\left(\mathfrak{g}_{1}\right) \rightarrow U(\mathfrak{g})$ e $\phi_{2}: U\left(\mathfrak{g}_{2}\right) \rightarrow U(\mathfrak{g})$ tais que $\phi_{1} \circ \pi_{1}=\pi \circ P_{1}$ e $\phi_{2} \circ \pi_{2}=\pi \circ P_{2}$. Pelo Corolário 1.3.15, temos que $\pi \circ P_{1}$ e $\pi \circ P_{2}$ são injetores e, portanto, $\phi_{1}$ e $\phi_{2}$ também são. O comutador $\left[x_{1}, x_{2}\right]=0$ para todo $x_{1} \in \mathfrak{g}_{1}$ e $x_{2} \in \mathfrak{g}_{2}, \operatorname{logo} \phi_{1}(x)$ e $\phi_{2}(x)$ comuta para todo $x \in \mathfrak{g}$. Sendo assim, a aplicação

$$
\begin{gathered}
\Phi: U\left(\mathfrak{g}_{1}\right) \otimes U\left(\mathfrak{g}_{2}\right) \rightarrow U(\mathfrak{g}) \\
x_{1} \otimes x_{2} \longmapsto \phi_{1}\left(x_{1}\right) \phi_{2}\left(x_{2}\right)
\end{gathered}
$$

é um homomorfismo de álgebras injetor.

Para mostrar que $\Phi$ é isomorfismo, considere $f: \mathfrak{g} \rightarrow U\left(\mathfrak{g}_{1}\right) \otimes U\left(\mathfrak{g}_{2}\right)$ dada por

$$
f\left(x_{1}, x_{2}\right)=\pi_{1}\left(x_{1}\right) \otimes 1+1 \otimes \pi_{2}\left(x_{2}\right) .
$$

Pela propriedade universal, temos que existe um homomorfismo de álgebras $F: U(\mathfrak{g}) \rightarrow U\left(\mathfrak{g}_{1}\right) \otimes$ $U\left(\mathfrak{g}_{2}\right)$ tal que $F \circ \pi=f$. Note que $\Phi\left(\sigma_{1}\left(x_{1}\right) \otimes 1+1 \otimes \sigma_{2}\left(x_{2}\right)\right)=x_{1}+x_{2}$ e, portanto, $\Phi \circ F \circ \pi=$ $\Phi \circ f=\pi$, implicando que $\Phi \circ F$ é a identidade de $U(\mathfrak{g})$. Por outro lado, $F \circ \Phi \circ f=F \circ \pi=f$, implicando que $F \circ \Phi$ é a identidade de $U\left(\mathfrak{g}_{1}\right) \otimes U\left(\mathfrak{g}_{2}\right)$.

Observação 1.3.20. Pela propriedade universal de $U(\mathfrak{g})$, toda representação $\rho: \mathfrak{g} \rightarrow \mathfrak{g l}(V)$ admite um único homomorfismo de álgebras $\hat{\rho}: U(\mathfrak{g}) \rightarrow \mathfrak{g l}(V)$ tal que $\hat{\rho} \circ \pi=\rho$. Portanto, $V$ é um $U(\mathfrak{g})$ módulo. Por outro lado, se $V$ é espaço vetorial que é um $U(\mathfrak{g})$-módulo, então podemos definir uma 
representação $\rho: \mathfrak{g} \rightarrow \mathfrak{g l}(V)$ dada por $\rho(x) v=\pi(x) \cdot v$. Sendo assim, existe uma relação biunívoca entre as representações de $\mathfrak{g}$ e os módulos sobre $U(\mathfrak{g})$. Portanto, a partir de agora, raramente diferenciaremos os dois objetos.

Proposição 1.3.21 (Teorema da Densidade de Jacobson). Seja $\mathfrak{g}$ uma álgebra de Lie e $V$ um $\mathfrak{g}$-módulo simples de dimensão enumerável. Suponha que $\mathfrak{g}$ possua dimensão finita ou $\mathbb{k}$ não seja um conjunto enumerável ou $V$ tem dimensão finita. Seja $\left\{v_{1}, \ldots, v_{l}\right\} \subset V$ um conjunto linearmente independente, então existe $u \in U(\mathfrak{g})$ tal que

$$
u v_{i}=\delta_{1 i} v_{1} \quad \text { para todo } i=1, \ldots, l .
$$

Demonstração. O Teorema da Densidade de Jacobson (veja Theorem 14.15 de [Isa09], originalmente publicado em [Jac45]) diz que se $R$ é um anel associativo com unidade, $M$ é um $R$-módulo simples, $D=\operatorname{End}_{R}(M)$, então, para todo $x_{1}, \ldots, x_{k} \in M$ elementos $D$-linearmente independente e $\varphi \in$ $\operatorname{End}_{D}(M)$, temos que existe $r \in R$ tal que $r \cdot x_{i}=\varphi\left(x_{i}\right)$, para $i=1, \ldots, r$.

Suponha $R=U(\mathfrak{g})$ e $M=V$. Seja $\left\{v_{1}, \ldots, v_{l}\right\} \subset V$ um conjunto linearmente independente. Pelo Lema de Schur (Lema 1.3.5), $D=\operatorname{End}_{\mathfrak{g}}(V) \cong \mathbb{k}$. Seja

$$
\varphi \in \operatorname{End}_{D}(V)=\operatorname{End}_{\operatorname{End}_{\mathfrak{g}}(V)}(V) \cong \operatorname{End}_{\mathbb{k}}(V)
$$

tal que $\varphi\left(v_{i}\right)=\delta_{i 1} v_{i}, i=1, \ldots, r$. Pelo Teorema da Densidade de Jacobson, existe $u \in U(\mathfrak{g})$ tal que $u v_{i}=\varphi\left(v_{i}\right)=\delta_{i 1} v_{i}^{1}$.

A hipótese que $\mathfrak{g}$ possua dimensão finita ou k não seja um conjunto enumerável é necessária para que possamos usar o segundo e terceiro item do Lema 1.3.5 (Lema de Schur).

Proposição 1.3.22. Sejam $\mathfrak{g}_{1}$ e $\mathfrak{g}_{2}$ álgebras de Lie. Sejam $V_{1}$ e $V_{2}$ módulos simples sobre $\mathfrak{g}_{1}$ e $\mathfrak{g}_{2}$, respectivamente. Suponha que $\mathfrak{g}_{1}$ possua dimensão finita ou $\mathbb{k}$ não seja um conjunto enumerável ou $V$ possua dimensão finita. Se $V_{1}$ possui dimensão enumerável, então $V_{1} \otimes V_{2}$ é um $\mathfrak{g}_{1} \oplus \mathfrak{g}_{2}$-módulo simples, onde a ação é induzida por

$$
\left(x_{1}+x_{2}\right)\left(v_{1} \otimes v_{2}\right)=\left(x_{1} v_{1}\right) \otimes v_{2}+x_{1} \otimes\left(x_{2} v_{2}\right), \quad x_{1} \in \mathfrak{g}_{1}, x_{2} \in \mathfrak{g}_{2}, v_{1} \otimes v_{2} \in V_{1} \otimes V_{2} .
$$

Demonstração. Pela Observação 1.3.7, temos que $V_{1} \otimes V_{2}$ é um $\mathfrak{g}_{1} \oplus \mathfrak{g}_{2}$-módulo. Para mostrar que $V_{1} \otimes V_{2}$ é simples, basta provar que para todo $v \in V_{1} \otimes V_{2}$, temos que $U\left(\mathfrak{g}_{1} \oplus \mathfrak{g}_{2}\right) v=V_{1} \otimes V_{2}$. Seja

$$
0 \neq v=\sum_{i=1}^{r} v_{i}^{1} \otimes v_{i}^{2} \in V_{1} \otimes V_{2}
$$

Podemos supor, sem perda de generalidade, que $\left\{v_{i}^{1}\right\}_{i=1}^{r}$ é um conjunto $\mathbb{k}$-linearmente independente e $v_{i}^{2} \neq 0$, para todo $i=1, \ldots, r$. Como $V_{1}$ possui dimensão enumerável, pelo Teorema 1.3.21, existe $u \in U\left(\mathfrak{g}_{1}\right)$ tal que $u v_{i}^{1}=\delta_{i 1} v_{i}^{1}$. Logo, $u \in U\left(\mathfrak{g}_{1} \oplus \mathfrak{g}_{2}\right) \mathrm{e}$

$$
u v=\sum_{i=1}^{r}\left(u v_{i}^{1}\right) \otimes v_{i}^{2}=v_{1}^{1} \otimes v_{1}^{2} .
$$

Seja $v_{1} \in V$ e $v_{2} \in V$ quaisquer elementos, então existem $u_{1} \in U\left(\mathfrak{g}_{1}\right) \subset U\left(\mathfrak{g}_{1} \oplus \mathfrak{g}_{2}\right)$ e $u_{2} \in$ $U\left(\mathfrak{g}_{2}\right) \subset U\left(\mathfrak{g}_{1} \oplus \mathfrak{g}_{2}\right)$ tais que $u_{1} v_{1}^{1}=v_{1}$ e $u_{2} v_{1}^{2}=v_{2}$. Logo,

$$
\begin{aligned}
u_{1}\left(\left(u_{2}(u v)\right)\right) & =u_{1}\left(u_{2}\left(v_{1}^{1} \otimes v_{1}^{2}\right)\right) \\
& =u_{1}\left(v_{1}^{1} \otimes\left(u_{2} v_{1}^{2}\right)\right) \\
& =u_{1}\left(v_{1}^{1} \otimes v_{2}\right) \\
& =\left(u_{1} v_{1}^{1}\right) \otimes v_{2}=v_{1} \otimes v_{2}
\end{aligned}
$$

Sendo assim, $v_{1} \otimes v_{2} \in U\left(\mathfrak{g}_{1} \oplus \mathfrak{g}_{2}\right) v$ para todo $v_{1} \otimes v_{2} \in V_{1} \otimes V_{2}$. Portanto, $V_{1} \otimes V_{2}=U\left(\mathfrak{g}_{1} \oplus \mathfrak{g}_{2}\right) v$. 
Observação 1.3.23. A Proposição 1.3.22 também é valida se supormos que $\mathfrak{g}_{1}$ é uma álgebra de Lie de dimensão finita e $V_{1}$ é um $\mathfrak{g}_{1}$-módulo simples qualquer. Basta ver que se $v_{1} \in V_{1}$ não é nulo, temos que $V_{1}=U\left(\mathfrak{g}_{1}\right) v_{1}$. Como $U\left(\mathfrak{g}_{1}\right)$ possui base enumerável, pois $\mathfrak{g}_{1}$ possui dimensão finita, então $V_{1}$ possui base enumerável também.

\subsubsection{Representações de Peso}

Os resultados e provas desta seção são baseados nos resultados apresentados e provados em [MP95] e [BLL15].

Definição 1.3.24. Seja $\mathfrak{h}$ uma álgebra de Lie abeliana e seja $\rho: \mathfrak{h} \rightarrow \mathfrak{g l}(V)$ uma representação de $\mathfrak{h}$. Um vetor não nulo $v \in V$ é chamado de vetor de peso $\lambda \in \mathfrak{h}^{*}$, se para todo $h \in \mathfrak{h}$ temos $\pi(h) v=\lambda(h) v$. Se $\lambda \in \mathfrak{h}^{*}$ é tal que

$$
V_{\lambda}=\{v \in V: \rho(h) v=\lambda(h) v, \text { para todo } v \in V\}
$$

é não nulo, então chamamos $V_{\lambda}$ de $\lambda$-espaço de peso (ou espaço de peso $\lambda$ ) e $\lambda$ é chamado de peso. Para cada $\lambda \in \mathfrak{h}^{*}$, claramente que $V_{\lambda}$ é um $\mathfrak{h}$-submódulo de $V$. Dizemos que $V$ admite uma decomposição em espaços de peso para $\mathfrak{h}$ se $V=\sum_{\lambda \in \mathfrak{h}^{*}} V^{\lambda}$. O conjunto de todos os pesos de $V$ será denotado por $\Pi(V)$.

Lema 1.3.25. Seja $\mathfrak{h}$ uma álgebra de Lie abeliana e $\rho: \mathfrak{h} \rightarrow \mathfrak{g l}(V)$ uma representação de $\mathfrak{h}$ tal que $V$ é admite uma decomposição em espaços de peso. Então a soma $V=\sum_{\lambda \in \mathfrak{h} *} V_{\lambda}$ é direta e qualquer submódulo $W \subset V$ é da forma $W=\sum_{\lambda \in \mathfrak{h}^{*}}\left(W \cap V_{\lambda}\right)$; isto é, $W$ também admite uma decomposição em espaços de peso.

Demonstração. Seja $0 \neq w \in W$. Como $W \subset V$ e $V=\sum_{\lambda \in \mathfrak{h}^{*}} V_{\lambda}$, segue que $w=v_{\lambda_{1}}+\cdots+v_{\lambda_{k}}$, onde $v_{\lambda_{i}} \in V_{\lambda_{i}}$ e $\lambda_{i} \neq \lambda_{j}$, se $i \neq j$. Queremos provar que cada $v_{\lambda_{i}}$ é um elemento de $W$. Provaremos por indução em $k$. Se $k=1$, segue $w \in V_{\lambda_{1}}$. Suponha $k>1$. Seja $h \in \mathfrak{h}$ tal que $\lambda_{1}(h) \neq \lambda_{2}(h)$ e $\lambda_{1}(h)=1$. Por hipótese, $\rho(h) w \in W$, porém $\rho(h) w=\lambda_{1}(h) v_{\lambda_{1}}+\cdots+\lambda_{k}(h) v_{\lambda_{k}}$ e

$$
(1-\rho(h)) w=\left(1-\lambda_{2}(h)\right) v_{2}+\cdots+\left(1-\lambda_{k}(h)\right) v_{k} \neq 0 .
$$

Utilizando a hipótese de indução em $(1-\rho(h))) w \in W, v_{\lambda_{2}} \in W$, implicando que $w-v_{\lambda_{2}}=v_{\lambda_{1}}+$ $v_{\lambda_{3}}+v_{\lambda_{4}}+\cdots+v_{\lambda_{k}} \in W$. Novamente, pela hipótese de indução, temos que $v_{\lambda_{1}}, v_{\lambda_{3}}, v_{\lambda_{4}}, \ldots, v_{\lambda_{k}} \in W$.

Utilizando a mesma prova acima porém supondo $W=0$ e $w=0$, temos que se $v_{\lambda_{1}}+\cdots+v_{\lambda_{k}}=0$, então $v_{\lambda_{1}}, \ldots, v_{\lambda_{k}}=0$. Ou seja, a soma $V=\sum_{\lambda \in \mathfrak{h}^{*}} V_{\lambda}$ é direta.

Definição 1.3.26. Seja $\mathfrak{g}$ uma álgebra de Lie e $\mathfrak{h} \subset \mathfrak{g}$ uma subálgebra abeliana de dimensão finita de $\mathfrak{g}$. Dizemos que $(\mathfrak{g}, \mathfrak{h})$ é um par válido, se $\mathfrak{h}$ é não nula e $\mathfrak{g}$ admite uma decomposição em espaços de peso.

Exemplo 1.3.27. Seja $\mathfrak{g}$ um álgebra de Lie semissimples, $\mathfrak{h}$ uma subálgebra de Cartan e $\Phi$ o sistema de raízes associado, então $\mathfrak{g}=\mathfrak{h} \oplus \bigoplus_{\alpha \in \Phi} \mathfrak{g}_{\alpha}$ é uma decomposição de $\mathfrak{g}$ em espaços em relação a $\mathfrak{h}$, portanto $(\mathfrak{g}, \mathfrak{h})$ é um par válido. Se $R$ é uma álgebra de Lie redutível tal que $R=\mathfrak{g} \oplus Z$, onde $Z$ é uma álgebra de Lie abeliana, então $H=\mathfrak{h} \oplus Z$ é uma subálgebra abeliana de $R$ e $(R, H)$ é um par válido.

Exemplo 1.3.28. Seja $\mathfrak{g}$ uma álgebra de Lie semissimples e $\mathfrak{h}$ uma subálgebra de Cartan de $\mathfrak{g}$. Seja $A$ uma álgebra comutativa associativa com unidade, então $\mathcal{G}=\mathfrak{g} \otimes A$ é uma álgebra de Lie, onde o comutador é definido por

$$
[x \otimes a, y \otimes b]=[x, y] \otimes a b, \quad x, y \in \mathfrak{g}, a, b \in A .
$$

Seja $\alpha$ uma raiz de $\mathfrak{g}$ e $x \in \mathfrak{g}_{\alpha}$, então

$$
[h \otimes 1, x \otimes a]=\alpha(h) x \otimes a,
$$


para todo $x \otimes a \in \mathfrak{g}_{\alpha} \otimes A$. Logo, $\mathcal{G}=(\mathfrak{h} \otimes A) \bigoplus_{\alpha \in \Phi}\left(g_{\alpha} \otimes A\right)$, onde $\Phi$ é o conjunto das raízes de $\mathfrak{g}$. Sendo assim, $(\mathcal{G}, \mathfrak{h} \otimes \mathbb{k})$ é um par válido.

Exemplo 1.3.29 (Âlgebra de Virasoro e Álgebra de Witt). A álgebra de Witt $W=\operatorname{Der} \mathbb{C}\left[t, t^{-1}\right]$ possui base $L_{s}=-t^{s+1} \frac{d}{d t}, s \in \mathbb{Z}$, e colchete de Lie dado por

$$
\left[L_{r}, L_{s}\right]=(r-s) L_{r+s} .
$$

Seja $H=\operatorname{span}_{\mathbb{C}} L_{0}$, então $W$ possui uma decomposição em espaços de peso em relação a $H$. Portanto, $(W, H)$ é um par válido.

Considere a extensão central $V=W \oplus \mathbb{C} c$ de $W$, dada por $\left[c, L_{n}\right]=0$ e

$$
\left[L_{r}, L_{s}\right]=(r-s) L_{r+s}+\frac{c}{12}\left(r^{3}-r\right) \delta_{r,-s},
$$

então $(V, H \oplus \mathbb{C} c)$ é um par válido. A álgebra de Lie $V$ é conhecida como álgebra de Virasoro.

Proposição 1.3.30. Seja $\mathfrak{g}$ uma álgebra de Lie e $\rho: \mathfrak{h} \rightarrow \operatorname{Der}(\mathfrak{g})$ um homomorfismo de álgebras de Lie, onde $\mathfrak{h}$ é uma álgebra de Lie abeliana e Der $(\mathfrak{g})$ está sendo considerado como a álgebra de Lie criada a partir das derivações de $\mathfrak{g}$.

1. Suponha que $\mathfrak{g}$ admita uma decomposição em espaços de peso

$$
\mathfrak{g}=\bigoplus_{\lambda \in \mathfrak{h}^{*}} \mathfrak{g}_{\lambda}
$$

com respeito a representação $\rho$ de $\mathfrak{h}$. Então,

(a) para todo $\lambda, \mu \in \mathfrak{h}^{*},\left[\mathfrak{g}_{\lambda}, \mathfrak{g}_{\mu}\right] \subset \mathfrak{g}_{\alpha+\mu}$;

(b) $U(\mathfrak{g})$ admite uma decomposição em espaços de peso relativa a representação induzida por $\rho$ e para todo $\lambda, \mu \in \mathfrak{h}$

$$
U(\mathfrak{g})_{\lambda} U(\mathfrak{g})_{\mu} \subset U(\mathfrak{g})_{\lambda+\mu} .
$$

2. Suponha que $\mathfrak{g}$ é gerada como uma álgebra de Lie por um conjunto $\left\{x_{j} \in \mathfrak{g}: j \in J\right\}$ e que, para cada $j \in J$, exista $\lambda_{j} \in \mathfrak{h}^{*}$ tal que $\rho(h) x_{j}=\lambda_{j}(h) x_{j}$. Então $\mathfrak{g}$ admite uma decomposição em espaços de pesos e $x_{j} \in g_{\lambda_{j}}$ e cada peso $\lambda \in \sum_{j \in J} \mathbb{Z} \lambda_{j}$.

Em particular, ambas afirmações valem quando $(\mathfrak{g}, \mathfrak{h})$ é um par válido, isto é, $\rho(h)=\mathrm{ad}_{h}$, para todo $h \in \mathfrak{h}$.

Demonstração. Cada elemento de $U(\mathfrak{g})$ é soma de produtos $x_{1} \ldots x_{k}$, onde $x_{i} \in \mathfrak{g}$. Suponha que $x_{i} \in \mathfrak{g}_{\lambda_{i}}$, então, para todo $h \in \mathfrak{h}$,

$$
\begin{aligned}
\rho(h)\left(x_{1} \ldots x_{k}\right) & =\sum_{j=1}^{k} x_{1} \ldots \rho(h) x_{j} \ldots x_{k} \\
& \sum_{j=1}^{k} x_{1} \ldots \lambda_{j}(h) x_{j} \ldots x_{k} \\
& =\left(\lambda_{1}+\cdots+\lambda_{k}\right)(h) x_{1} \ldots x_{k} .
\end{aligned}
$$

Em particular, se $x \in \mathfrak{g}_{\lambda}$ e $y \in \mathfrak{g}_{\mu}$, temos que $\rho(h)[x, y] \in \mathfrak{g}_{\lambda+\mu}$. Além disso, $U(\mathfrak{g})$ admite uma decomposição em espaços de peso e $U(\mathfrak{g})_{\lambda} U(\mathfrak{g})_{\mu} \subset U(\mathfrak{g})_{\lambda+\mu}$.

Se $\left\{x_{j} \in \mathfrak{g}: j \in J\right\}$ gera $\mathfrak{g}$ como uma álgebra de Lie, então a conta acima também mostra que $U(\mathfrak{g})$ admite uma decomposição em espaços de pesos. Como podemos ver $\mathfrak{g}$ como um $\mathfrak{h}$-submódulo de $U(\mathfrak{g})$, então $\mathfrak{g}$ também admite uma decomposição em espaços de peso. 
Definição 1.3.31. Seja $(\mathfrak{g}, \mathfrak{h})$ um par de álgebras de Lie válido e $V$ um $\mathfrak{g}$-módulo. Dizemos que $V$ é um $\mathfrak{g}$-módulo de peso se $V$ admite uma decomposição em espaços de pesos sobre $\mathfrak{h}$ e, semelhantemente, realizamos a Definição $1.3 .24 \mathrm{em} V$. Suponha que $V$ é um g-módulo de peso. Dizemos que $V$ é quasi-finito, se para todo $\lambda \in \Pi(V)$ temos que $V_{\lambda}$ possui dimensão finita e $\Pi(V)$ possui cardinalidade enumerável. Dizemos que $V$ é uniformemente limitado, se $V$ é um g-módulo quasi-finito tal que exista $M \in \mathbb{Z}_{>0}$ tal que $\operatorname{dim}\left(V_{\lambda}\right)<M$, para todo $\lambda \in \Pi(V)$.

Se $(\mathfrak{g}, \mathfrak{h})$ é um par de álgebras de Lie válido e $V$ é um $\mathfrak{g}$-módulo quasi-finito simples, então a dimensão de $V$ é igual $\sum_{\lambda \in \Pi(V)} \operatorname{dim}\left(V_{\lambda}\right)$. Logo, como $\operatorname{dim}\left(V_{\lambda}\right)$ é finita para todo $\lambda \in \Pi(V)$ e $\Pi(V)$ possui cardinalidade enumerável, então $V$ possui dimensão enumerável. Em particular, se g possui dimensão enumerável, então $U(\mathfrak{g})$ possui dimensão enumerável. Neste caso, $W=U(\mathfrak{g}) w$ possui dimensão enumerável também, onde $W$ é um g-módulo simples e $w \in W$ não é nulo. Logo, se $\mathfrak{g}$ possui dimensão enumerável e $W$ é um $\mathfrak{g}$-módulo de peso simples tal que para todo $\lambda \in \Pi(W)$ temos que $W_{\lambda}$ possui dimensão finita, então $W$ é um g-módulo quasi-finito.

Lema 1.3.32. Sejam $(\mathfrak{g}, \mathfrak{h})$ um par válido de álgebras de Lie e $V$ um $\mathfrak{g}$-módulo de quasi-finito. Suponha que exista $\lambda \in \mathfrak{h}^{*}$ tal que o espaço

$$
W_{\lambda}=\{w \in W \mid h w=\lambda(h) w \text { para todo } h \in \mathfrak{h}\}
$$

seja não nulo para todo submódulo não nulo $W \subset V$, então $V$ contém um $\mathfrak{g}$-submódulo simples.

Demonstração. Seja $W \subset V$ um g-submódulo não nulo tal que a dimensão de $W_{\lambda}$ seja minimal. Considere $M=U(\mathfrak{g}) W_{\lambda}$. Para todo $m \in M$ não nulo, $(U(\mathfrak{g}) m)_{\lambda}$ é não nulo, por hipótese. Como a dimensão de $W_{\lambda}$ é minimal, temos que $(U(\mathfrak{g}) m)_{\lambda}=W_{\lambda}$. Sendo assim, $W_{\lambda} \subset U(\mathfrak{g}) m \subset M$. Logo, $U(\mathfrak{g}) m=M$ e $M$ é simples.

Lema 1.3.33. Sejam $\left(\mathfrak{g}_{1}, \mathfrak{h}_{1}\right)$ e $\left(\mathfrak{g}_{2}, \mathfrak{h}_{2}\right)$ pares válidos de álgebras de Lie, então as somas diretas de álgebras de Lie formam o par válido $\left(\mathfrak{g}_{1} \oplus \mathfrak{g}_{2}, \mathfrak{h}_{1} \oplus \mathfrak{h}_{2}\right)$. Suponha que $V$ seja um módulo quasi-finito sobre $\mathfrak{g}_{1} \oplus \mathfrak{g}_{2}$. Seja $v \in V$ um vetor não nulo de peso $(\lambda, \mu) \in \mathfrak{h}_{1}^{*} \times \mathfrak{h}_{2}^{*} \cong\left(\mathfrak{h}_{1} \oplus \mathfrak{h}_{2}\right)^{*}$, então $U\left(\mathfrak{g}_{1}\right) v$ é um $\mathfrak{g}_{1}$-módulo quasi-finito e $\operatorname{Hom}_{\mathfrak{g}_{1}}(M, V)$ é um $\mathfrak{g}_{2}$-módulo quasi-finito, para todo submódulo $M \subset U\left(\mathfrak{g}_{1}\right) v$.

Demonstração. Como $\mathfrak{h}_{1}$ e $\mathfrak{h}_{2}$ possuem dimensão finita, temos que $\mathfrak{h}_{1} \oplus \mathfrak{h}_{2}$ possui dimensão finita e $\left(\mathfrak{h}_{1} \oplus \mathfrak{h}_{2}\right)^{*} \cong \mathfrak{h}_{1}^{*} \times \mathfrak{h}_{2}^{*}$. Como $\mathfrak{h}_{1} \oplus \mathfrak{h}_{2} \subset \mathfrak{g}_{1} \oplus \mathfrak{g}_{2}$ é uma subálgebra de Lie abeliana não nula, temos que $\left(\mathfrak{g}_{1} \oplus \mathfrak{g}_{2}, \mathfrak{h}_{1} \oplus \mathfrak{h}_{2}\right)$ é um par válido.

Seja $v \in V$ um vetor não nulo de peso $(\lambda, \mu) \in \mathfrak{h}_{1}^{*} \times \mathfrak{h}_{2}^{*}$ e $W=U\left(\mathfrak{g}_{1}\right) v \subset V$. Temos que $V$ é um $\mathfrak{h}_{1}$-módulo de peso $W$ é um $\mathfrak{h}_{1}$-submódulo, logo $W$ é um $\mathfrak{g}_{1}$-módulo de peso pelo Lema 1.3.25. Para cada $h_{2} \in \mathfrak{h}_{2}$ e $x \in \mathfrak{g}_{1}$, temos que

$$
h_{2} x v=x h_{2} v+[h, x] v=\mu(h) x v .
$$

Logo, se $u \in U\left(\mathfrak{g}_{1}\right)$ é tal que $u v \in W_{\eta}$, temos que $u v \in V_{(\eta, \mu)}$. Ou seja, $W_{\eta} \subset V_{(\eta, \mu)}$, para todo $\eta \in \mathfrak{h}_{1}^{*}$. Sendo assim, $W$ é um $\mathfrak{g}_{1}$-módulo quasi-finito, assim como todo $\mathfrak{g}_{1}$-submódulo $M \subset W$.

Seja $M$ um g-submódulo de $W$ e $I \subset U\left(\mathfrak{g}_{1}\right)$ tal que $I=\left\{u \in U\left(\mathfrak{g}_{1}\right) \mid u v \in M\right\}$. Como $(x u) v=x(u v) \in M$, temos que $x u \in I$, para todo $u \in I$ e $x \in U\left(\mathfrak{g}_{1}\right)$. Logo, $I$ é um ideal (à esquerda) de $U\left(\mathfrak{g}_{1}\right)$. Note que $\operatorname{Hom}_{\mathfrak{g}_{1}}(M, V)$ admite uma estrutura de $\mathfrak{g}_{2}$-módulo com a ação

$$
(x \varphi)(m)=x(\varphi(m)) \text {, onde } x \in \mathfrak{g}_{2}, \varphi \in \operatorname{Hom}_{\mathfrak{g}_{1}}(M, V) \text { e } m \in M .
$$

Seja $\varphi \in \operatorname{Hom}_{\mathfrak{g}_{1}}(M, V)$, então $h_{2} \varphi(u v)=h_{2} u \varphi(v)=u\left(h_{2}(\varphi(m))\right)$, para todo $u \in I$ e $h_{2} \in \mathfrak{h}_{2}$. Além disso, $h_{1} \varphi(v)=\lambda\left(h_{1}\right) \varphi(v)$, para todo $h_{1} \in \mathfrak{h}_{1}$, isto é, $\varphi(v) \in \bigoplus_{\gamma \in \mathfrak{h}_{2}^{*}} V_{(\lambda, \gamma)}$. Logo, existem $v_{1}, \ldots, v_{k} \in V$ e $\gamma_{1}, \ldots, \gamma_{k} \in \mathfrak{h}_{2}^{*}$ tais que $0 \neq v_{i} \in V_{\left(\lambda, \gamma_{i}\right)}, i=1 \ldots, k$, e $\varphi(v)=v_{1}+\cdots+v_{k}$. Para cada $i=1, \ldots, k$, considere $\varphi_{i}: M \rightarrow V$ dada por $\varphi_{i}(u v)=u v_{i}$ para todo $u \in I$, então $\varphi_{i}$ é uma transformação linear e

$$
\varphi_{i}(x u v)=x u v_{i}=x \varphi_{i}(u v),
$$


para todo $u \in I$ e $x \in \mathfrak{g}_{1}$. Logo, $\varphi_{i} \in \operatorname{Hom}_{\mathfrak{g}_{1}}(M, V)$. Além disso,

$$
\left(h_{2} \varphi_{i}\right)(u v)=u h_{2} v_{i}=\gamma_{i}\left(h_{2}\right) u v_{i}=\gamma_{i}\left(h_{2}\right) \varphi_{i}(u v),
$$

para todo $u \in I$ e $h_{2} \in \mathfrak{h}_{2}$, portanto $\varphi_{i} \in \operatorname{Hom}_{\mathfrak{g}_{1}}(M, V)_{\gamma_{i}}$. Temos ainda, para todo $u \in I$,

$$
\begin{aligned}
\varphi(u v)=u \varphi(v) & =u\left(v_{1}+\cdots+v_{k}\right) \\
& =u\left(\varphi_{1}(v)+\cdots+\varphi_{k}(v)\right) \\
& =\varphi_{1}(u v)+\cdots+\varphi_{k}(u v),
\end{aligned}
$$

portanto $\varphi=\varphi_{1}+\cdots+\varphi_{k} \in \bigoplus_{i=1}^{k} \operatorname{Hom}_{\mathfrak{g}_{1}}(M, V)_{\gamma_{i}}$. Concluímos que

$$
\operatorname{Hom}_{\mathfrak{g}_{1}}(M, V)=\bigoplus_{\gamma \in \mathfrak{h}_{2}^{*}} \operatorname{Hom}_{\mathfrak{g}_{1}}(M, V)_{\gamma}
$$

é um $\mathfrak{g}_{2}$-módulo de peso.

Falta mostrar que os espaços de peso de $\operatorname{Hom}_{\mathfrak{g}_{1}}(M, V)$ possuem dimensão finita. Fixe $\gamma \in \mathfrak{h}_{2}^{*}$ e uma base $w_{1}, \ldots, w_{l}$ de $V_{(\lambda, \gamma)}$. Como $\psi(u v)=u \psi(v)$ para todo $u \in I, \psi$ estará completamente definido se soubermos o valor de $\psi(v)$, onde $\psi \in \operatorname{Hom}_{\mathfrak{g}_{1}}(M, V)_{\gamma}$ é qualquer. Sendo assim, para cada $i=1, \ldots, l$, existe um único $\psi_{i} \in \operatorname{Hom}_{\mathfrak{g}_{1}}(M, V)_{\gamma}$ tal que $\psi_{i}(v)=w_{i}$. Logo, para todo $\psi \in \operatorname{Hom}_{\mathfrak{g}_{1}}(M, V)_{\gamma}$ existem únicos $a_{1}, \ldots, a_{l} \in \mathbb{k}$ tais que

$$
\psi(v)=\sum_{i=1}^{l} a_{i} w_{i}=\sum_{i=1}^{l} a_{i} \psi_{i}(v) .
$$

Como $\psi(u v)=\sum_{i=1}^{l} a_{i} \psi_{i}(u v)$ para todo $u \in I$, então $\psi_{1}, \ldots, \psi_{k}$ é uma base de $\operatorname{Hom}_{\mathfrak{g}_{1}}(M, V)_{\gamma}$, implicando que $\operatorname{Hom}_{\mathfrak{g}_{1}}(M, V)$ é um $\mathfrak{g}_{2}$-módulo quasi-finito.

A seguinte proposição foi provada em [BLL15], porém supondo que $\mathfrak{g}_{1}$ e $\mathfrak{g}_{2}$ são álgebras de Lie semissimples e $\mathfrak{h}_{1} \subset \mathfrak{g}_{1}$ e $\mathfrak{h}_{2} \subset \mathfrak{g}_{2}$ são subálgebras de Cartan. Adaptamos a prova para o caso mais geral de que $\left(\mathfrak{g}_{1}, \mathfrak{h}_{1}\right)$ e $\left(\mathfrak{g}_{2}, \mathfrak{h}_{2}\right)$ são pares válidos de álgebras de Lie. Para isto precisaremos supor que uma das álgebras de Lie possui dimensão finita ou k seja um conjunto não enumerável, para que possamos usar a Proposição 1.3.22.

Proposição 1.3.34 ( [BLL15, Proposition 3.4]). Sejam $\left(\mathfrak{g}_{1}, \mathfrak{h}_{1}\right)$ e $\left(\mathfrak{g}_{2}, \mathfrak{h}_{2}\right)$ pares de álgebras de Lie válidos. Suponha que $\mathfrak{g}_{1}$ possua dimensão finita ou $\mathbb{k}$ seja um conjunto não enumerável. Suponha que $V$ seja um módulo quasi-finito simples sobre $\mathfrak{g}_{1} \oplus \mathfrak{g}_{2}$. Logo, $V \cong V_{1} \otimes V_{2}$, onde $V_{1}$ e $V_{2}$ são módulos quasi-finitos simples sobre $\mathfrak{g}_{1}$ e $\mathfrak{g}_{2}$, respectivamente.

Demonstração. Seja $v \in V$ um vetor não nulo de peso $(\lambda, \mu) \in \mathfrak{h}_{1}^{*} \times \mathfrak{h}_{2}^{*}$, então $W=U\left(\mathfrak{g}_{1}\right) v \subset V$ é um $\mathfrak{g}_{1}$-módulo quasi-finito e, portanto, todo $\mathfrak{g}_{1}$-submódulo $M \subset W$ é $\mathfrak{g}_{1}$-módulo quasi-finito. Seja $M \subset W$ um $\mathfrak{g}_{1}$-submódulo, não necessariamente próprio. Pelo Lema 1.3.33, $\operatorname{Hom}_{\mathfrak{g}_{1}}(M, V)$ é um $\mathfrak{g}_{2}$-módulo quasi-finito. Seja $N \subset \operatorname{Hom}_{\mathfrak{g}_{1}}(M, V)$ um $\mathfrak{g}_{2}$-submódulo não nulo, não necessariamente próprio. Como $M$ e $N$ não são nulos, temos que existe um $\varphi \in N$ não nulo e $m \in M$ tal que $\varphi(m) \neq 0$. Logo, a aplicação

$$
\begin{aligned}
\phi_{M, N}: M \times N & \rightarrow V \\
(m, \varphi) & \mapsto \varphi(m),
\end{aligned}
$$

é não nula. Note que

$$
\begin{aligned}
\phi_{M, N}\left(a m_{1}+m_{2}, b \varphi_{1}+\varphi_{2}\right) & =\left(b \varphi_{1}+\varphi_{2}\right)\left(a m_{1}+m_{2}\right) \\
& =a b \varphi_{1}\left(m_{1}\right)+b \varphi_{1}\left(m_{2}\right)+a \varphi_{2}\left(m_{1}\right)+\varphi_{2}\left(m_{2}\right) \\
& =a b \phi_{M, N}\left(m_{1}, \varphi_{1}\right)+b \phi_{M, N}\left(m_{2} \varphi_{1}\right)+\phi_{M, N}\left(m_{1}, \varphi_{2}\right)+\phi_{M, N}\left(m_{2}, \varphi_{2}\right),
\end{aligned}
$$


para todo $a, b \in \mathbb{k}, m_{1}, m_{2} \in M, \varphi_{1}, \varphi_{2} \in N$. Portanto, $\phi_{M, N}$ é bilinear e, pela propriedade universal do produto tensorial, existe uma única aplicação linear

$$
\begin{aligned}
\Phi_{M, N}: M \otimes N & \rightarrow V \\
(m, \varphi) & \mapsto \varphi(m) .
\end{aligned}
$$

Para todo $x \in \mathfrak{g}_{1}, y \in \mathfrak{g}_{2}$ e $m \otimes \varphi \in M \otimes N$, temos que

$$
\begin{aligned}
\Phi_{M, N}((x, y)(m \otimes \varphi)) & =\Phi_{M, N}((x m) \otimes \varphi+m \otimes(y \varphi)) \\
& =\varphi(x m)+(y \varphi)(m) \\
& =x(\varphi(m))+y(\varphi(m)) \\
& =(x+y) \varphi(m)=(x, y) \varphi(m)=(x, y) \Phi_{M, N}(m \otimes \varphi)
\end{aligned}
$$

Portanto, $\Phi_{M, N}$ é um homomorfismo de $\mathfrak{g}_{1} \oplus \mathfrak{g}_{2}$-módulos e é não nulo. Como $V$ é simples, segue que $\Phi_{M, N}$ é sobrejetor. Note que $(M \otimes N)_{(\alpha, \beta)}=M_{\alpha} \otimes N_{\beta}$ com $\alpha \in \mathfrak{h}_{1}^{*}$ e $\beta \in \mathfrak{h}_{2}^{*}$, portanto a restrição de $\Phi_{M, N}$ para $M_{\lambda} \otimes N_{\mu} \rightarrow V_{(\lambda, \mu)}$ é sobrejetora também, onde $M_{\lambda}, N_{\mu}$ e $V_{(\lambda, \mu)}$ são os espaços de peso $\lambda \in \mathfrak{h}_{1}^{*}, \mu \in \mathfrak{h}_{2}^{*}$ e $(\lambda, \mu) \in \mathfrak{h}_{1}^{*} \times \mathfrak{h}_{2}^{*}$. Como $V_{(\lambda, \mu)}$ é não nulo, segue que $M_{\lambda}$ e $N_{\mu}$ são não nulos.

Portanto, para todo submódulo $M \subset W$ temos que $M_{\lambda} \neq 0$. Pelo Lema 1.3.32, existe um $\mathfrak{g}_{1}$-submódulo não nulo $V_{1} \subset W$ de peso e simples. Além disso, para todo submódulo $N \subset$ $H_{o m} \mathfrak{g}_{1}\left(V_{1}, V\right)$ temos que $N_{\mu} \neq 0$. Pelo Lema 1.3.32, existe um $\mathfrak{g}_{2}$-submódulo não nulo $V_{2} \subset$ $H_{o m} \mathfrak{g}_{1}\left(V_{1}, V\right)$ de peso e simples. Logo, $V_{1} \otimes V_{2}$ é um $\mathfrak{g}_{1} \oplus \mathfrak{g}_{2}$-módulo de peso não nulo e, pela Proposição 1.3.22, é simples. Portanto, como $\Phi_{V_{1}, V_{2}}: V_{1} \otimes V_{2} \rightarrow V$ é uma aplicação sobrejetora não nula entre $\mathfrak{g}_{1} \oplus \mathfrak{g}_{2}$-módulos simples, temos que $\Phi_{V_{1}, V_{2}}$ é um isomorfismo.

Corolário 1.3.35. Seja $R$ uma álgebra de Lie redutivel de dimensão finita, isto é, $R=L \oplus Z$ é uma álgebra de Lie de dimensão finita, onde $L$ é uma álgebra de Lie semissimples e $Z$ é o centro de R. Seja $H$ uma subálgebra de Cartan de L. Seja $V$ um R-módulo quasi-finito simples, isto é, $V$ é um $R$-módulo simples tal que $V=\bigoplus_{(\lambda, \mu) \in H^{*} \times Z^{*}} V_{(\lambda, \mu)}$, onde

$$
V_{(\lambda, \mu)}=\{v \in V \mid(h+z) v=(\lambda(h)+\mu(z)) v, \quad h \in H, z \in Z\},
$$

então existe um L-módulo quasi-finito simples $W$ e um $\chi: Z \rightarrow \mathbb{k}$ tal que $W \cong V \otimes \mathbb{k}_{\chi}$, onde $\mathbb{k}_{\chi}$ é o $H$-módulo unidimensional com a ação dada por $\chi$. Em particular, se $L=L_{1} \oplus \cdots \oplus L_{k}$, onde $L_{i}$ são ideais de $L$ que são álgebras de Lie simples, então, para cada $i=1, \ldots, k$, existe um $L_{i}$-módulo quasi-finito simples $V_{i}$ tal que $V \cong V_{1} \otimes \cdots \otimes V_{k} \otimes \mathbb{k}_{\chi}$.

Demonstração. Pela Proposição 1.3.34, se $V$ é um $L \oplus H$-módulo simples, então é isomorfo à $W \otimes W^{\prime}$, onde $W$ e $W$ são módulos quasi-finitos simples para $L$ e $H$, respectivamente. Como $H$ é abeliano, todos os $H$-módulos simples são unidimensionais e a ação é dada por uma transformação linear $\chi: H \rightarrow \mathbb{k}$, então $W^{\prime} \cong \mathbb{k}_{\chi}$.

Se $L$ é uma álgebra de Lie semissimples, então $L=L_{1} \oplus \cdots \oplus L_{k}$, onde $L_{i}$ são ideais de $L$ que são álgebras de Lie simples. Pela Preposição 1.3.33, $W \cong V_{1} \oplus V^{\prime}$, onde $V_{1}$ é um $L_{1}$-módulos quasi-finito simples e $V^{\prime}$ é um $L_{2} \oplus \cdots \oplus L_{k}$-módulo quasi-finito simples, respectivamente. Como $L_{j} \oplus \cdots \oplus L_{k}$ é semissimples para todo $j=2, \ldots, k$, por recursão temos que $W \cong V_{1} \otimes \cdots \otimes V_{k}$, onde $V_{i}$ é um $L_{i}$-módulo quasi-finito simples, para cada $i=1, \ldots, k$.

Corolário 1.3.36. Se $\mathfrak{g}$ é um álgebra simples de dimensão finita com álgebra de Cartan $\mathfrak{h}$ e $V$ é $u m \mathfrak{g}^{\oplus r}$-módulo quasi-finito simples, em relação à álgebra de Cartan $\mathfrak{h}^{\oplus r}$, então $V \cong V_{1} \otimes \cdots \otimes V_{r}$, onde $V_{1}, \ldots, V_{r}$ são $\mathfrak{g}$-módulos quasi-finitos simples.

\subsection{Representações de Álgebras de Lie Semissimples}

Lembre que $k$ é um corpo algebricamente fechado de característica 0. Nesta seção, fixaremos uma álgebra de Lie semissimples $\mathfrak{g}$, uma subálgebra de Cartan $\mathfrak{h}$ de $\mathfrak{g}$ e $\Phi$ o sistema de raízes 
associado. Para cada $\alpha \in \Phi$, sejam $x_{\alpha} \in \mathfrak{g}_{\alpha}, x_{-\alpha} \in \mathfrak{g}_{\alpha}$ e $h_{\alpha} \in \mathfrak{h}$ tais que

$$
\left[x_{\alpha}, x_{-\alpha}\right]=h_{\alpha}, \quad\left[h_{\alpha}, x_{\alpha}\right]=2 x_{\alpha}, \quad\left[h_{\alpha}, x_{-\alpha}\right]=-2 x_{-\alpha} .
$$

Note que $h_{\alpha}=-h_{-\alpha}$. Seja $\Delta$ uma base de $\Phi$ e denote por $\Phi_{+}$e $\Phi_{-}$o conjunto das raízes positivas e negativas em relação à $\Delta$, respectivamente. Praticamente todos os resultados desta seção são resultados clássicos da teoria de representações da álgebras de Lie semissimples. Como feito na Seção 1.1, uma referência clássica é [Hum78].

\subsubsection{Módulos de Dimensão Finita e Módulos de Peso Máximo}

Lembre que $\mathfrak{g}$ admite uma decomposição triangular

$$
\mathfrak{g}=\mathfrak{g}_{-} \oplus \mathfrak{h} \oplus \mathfrak{g}_{+},
$$

onde $\mathfrak{g}_{ \pm}=\bigoplus_{\alpha \in \Phi_{ \pm}} \mathfrak{g}_{\alpha}$. O $\mathbb{Z}$-módulo $Q=\operatorname{span}_{\mathbb{Z}}\{\alpha \in \Phi\}$ é chamado de reticulado de raízes. Pela Proposição 1.3.30, a álgebra envolvente universal admite decomposição em espaços de peso para $\mathfrak{h}$ e seus pesos são exatamente $Q$. Como

$$
\left\{x_{-\alpha} \mid \alpha \in \Phi_{+}\right\} \cup\left\{\mathfrak{h}_{\alpha} \mid \alpha \in \Delta\right\} \cup\left\{x_{\alpha} \mid \alpha \in \Phi_{+}\right\}
$$

é uma base de $\mathfrak{g}$ e pelo Teorema de Poincaré-Birkhoff-Witt (Teorema PBW), temos que

$$
U(\mathfrak{g})=U\left(\mathfrak{g}_{-}\right) U(\mathfrak{h}) U\left(\mathfrak{g}_{+}\right) .
$$

A representação adjunta de $\mathfrak{h}$ em $\mathfrak{g}_{ \pm}$induz uma estrutura de $\mathfrak{h}$-módulo em $U\left(\mathfrak{g}_{ \pm}\right)$. Além disso, como os monômios da forma

$$
x_{ \pm \alpha_{1}}^{n_{1}} \ldots x_{ \pm \alpha_{k}}^{n_{k}}, \quad n_{1}, \ldots, n_{k} \in \mathbb{Z}_{+},
$$

onde $\Phi_{+}=\left\{\alpha_{1}, \ldots, \alpha_{k}\right\}$, formam uma base de $\mathfrak{g}_{ \pm}$, é possível mostrar, utilizando uma prova semelhante à dada na Proposição 1.3.30, que $U\left(\mathfrak{g}_{ \pm}\right)$admite decomposição em espaços de peso e seus pesos são exatamente os elementos de $Q_{ \pm}=\left\{\sum_{\alpha \in \Delta} a_{\alpha} \alpha \mid a_{\alpha} \in \mathbb{Z}_{ \pm}\right\}$.

Para classificar os g-módulos de dimensão finita, iremos começar enunciando um teorema clássico da teoria das representações de álgebras de Lie, o Teorema de Weyl.

Teorema 1.4.1 (Teorema de Weyl). Seja $\rho: \mathfrak{g} \rightarrow \mathfrak{g l}(V)$ uma representação de dimensão finita da álgebra de Lie semissimples $\mathfrak{g}$, então $\rho$ é completamente redutivel.

Demonstração. Veja Theorem 6.3 de [Hum78].

Isto é, toda representação de $\mathfrak{g}$ de dimensão finita é soma direta de representações irredutíveis. Portanto, classificando os g-módulos simples de dimensão finita, teremos a classificação de todos os $\mathfrak{g}$-módulos de dimensão finita.

Definição 1.4.2. Seja $V$ um g-módulo. Dizemos que $V$ é um g-módulo de peso, se $V$, visto como $\mathfrak{h}$-módulo, admite decomposição em espaços de peso. Denotaremos o conjunto de todos os pesos de $V$ por $\Pi(V)$. Dizemos que $V$ é um g-módulo de peso máximo $\Lambda \in \mathfrak{h}^{*}$, se existir $v_{\Lambda} \in V$ tal que

(i) $h v_{\Lambda}=\Lambda(h) v$, para todo $h \in \mathfrak{h}$;

(ii) $\mathfrak{g}_{+} v_{\Lambda}=0$;

(iii) $U(\mathfrak{g}) v_{\Lambda}=V$.

A subálgebra de $\mathfrak{g}$ definida por

$$
\mathfrak{b}=\mathfrak{h} \oplus \mathfrak{g}_{+}
$$


é chamada de subálgebra de Borel. Temos que $[\mathfrak{b}, \mathfrak{b}] \subset \mathfrak{g}_{+}$, pois $\mathfrak{h}$ é abeliana e $\mathfrak{g}_{+}$é invariante sobre a ação adjunta de $\mathfrak{h}$. Por outro lado, se $h \in \mathfrak{h}$ existe $\alpha \in \Phi$ tal que $\alpha(h) \neq 0$, então $\left[h, \mathfrak{g}_{\alpha}\right] \neq 0$, logo

$$
\mathfrak{g}_{+}=[\mathfrak{b}, \mathfrak{b}] .
$$

Como $\mathfrak{g}_{+}$é uma subálgebra de $\mathfrak{g}$ nilpotente, segue que $\mathfrak{b}$ é uma álgebra de Lie solúvel. Além disso, não existe uma subálgebra maximal solúvel de $\mathfrak{g}$ que contém $\mathfrak{b}$ propriamente.

Proposição 1.4.3. Seja $V$ um $\mathfrak{g}$-módulo simples de dimensão finita, então $V$ é um $\mathfrak{g}$-módulo de peso máximo.

Demonstração. Pelo Teorema de Lie (Teorema 1.2.8), $\mathfrak{b}$ possui um autovetor $0 \neq v \in V$ e $\hat{\Lambda} \in \mathfrak{b}^{*}$ tal que $b v=\hat{\Lambda}(b) v$, para todo $b \in \mathfrak{b}$. Para todo $b_{1}, b_{2} \in \mathfrak{b}$,

$$
\left[b_{1}, b_{2}\right] v=\hat{\Lambda}\left(\left[b_{1}, b_{2}\right]\right) v=\left(\hat{\Lambda}\left(b_{1}\right) \hat{\Lambda}\left(b_{2}\right)-\hat{\Lambda}\left(b_{2}\right) \hat{\Lambda}\left(b_{1}\right)\right) v=0 .
$$

Sendo assim, $\hat{\Lambda}\left(\mathfrak{g}_{+}\right)=0$. Seja $\Lambda=\left.\hat{\Lambda}\right|_{\mathfrak{h}} \in \mathfrak{h}^{*}$, então $h v=\Lambda(h) v$ para todo $h \in \mathfrak{h}$. Como vimos, $\mathfrak{g}_{+} v=0$. Por fim, $U(\mathfrak{g}) v$ é um submódulo não nulo de $V$, como $V$ é simples, temos que $V=U(\mathfrak{g}) v$.

Definição 1.4.4. Seja $V$ um g-módulo qualquer e seja $\lambda \in \mathfrak{h}^{*}$. Denotaremos por $V_{\lambda}=\{v \mid h v=$ $\lambda(h) v$ para todo $h \in \mathfrak{h}\}$. Além disso, um vetor não nulo $v \in V_{\lambda}$ é chamado chamado de vetor singular de peso $\lambda$ se $\mathfrak{g}_{+} v=0$. Se existe um vetor singular em $V_{\lambda}$, dizemos que $\lambda$ é chamado de peso singular de $V$. Se $v \in V_{\lambda}$ é um vetor singular e $V=U(\mathfrak{g}) v$, dizemos que $v$ é um vetor de peso máximo.

Dado $\Lambda \in \mathfrak{h}^{*}$, denotaremos por $D(\Lambda)=\left\{\Lambda-\sum_{\alpha \in \Delta} a_{\alpha} \alpha \mid a_{\alpha} \in \mathbb{Z}_{+}\right\} \subset \mathfrak{h}^{*}$ e $\Pi(V)=\left\{\lambda \in \mathfrak{h}^{*} \mid\right.$ $\left.V_{\lambda} \neq 0\right\}$.

Proposição 1.4.5. Seja $V$ um $\mathfrak{g}$-módulo de peso máximo com peso máximo $\Lambda$, então

(i) $V=\bigoplus_{\lambda \in D(\Lambda)} V_{\lambda}$, isto é, $V$ é um $\mathfrak{g}$-módulo de peso e $\Pi(V) \subset D(\Lambda)$;

(ii) $V_{\Lambda}=\mathbb{k} v_{\Lambda}$ e $\operatorname{dim} V_{\lambda}<\infty$, para todo $\lambda \in D(\Lambda)$;

(iii) $V$ é simples se, e somente se, $\Lambda$ é o único peso singular de $V$;

(iv) $V$ contém um único submódulo próprio maximal.

Demonstração. Seja $v_{\lambda}$ um vetor singular de peso $\Lambda$. Como $U(\mathfrak{b}) v_{\Lambda}=\mathbb{k} v_{\Lambda}$, temos

$$
\begin{aligned}
V & =U(\mathfrak{g}) v_{\Lambda} \\
& =U\left(\mathfrak{g}_{-}\right) U(\mathfrak{h}) U\left(\mathfrak{g}_{+}\right) v_{\Lambda} \\
& =U\left(\mathfrak{g}_{-}\right) v_{\Lambda} .
\end{aligned}
$$

Como $U\left(\mathfrak{g}_{-}\right)$admite decomposição em espaços de peso sobre $\mathfrak{h}$, temos que $V$ é gerado por vetores da forma $u v_{\Lambda}$, onde $u \in U\left(\mathfrak{g}_{-}\right)$é um vetor de peso. Seja $\alpha \in Q_{+}$e $u \in U\left(\mathfrak{g}_{-}\right)_{-\alpha}$, então, para todo $h \in \mathfrak{h}$,

$$
\begin{aligned}
h u v_{\Lambda} & =u h v_{\Lambda}+[h, u] v_{\Lambda} \\
& =\Lambda(h) u v_{\Lambda}-\alpha(h) u v_{\Lambda} \\
& =(\Lambda-\alpha)(h) u v_{\Lambda} .
\end{aligned}
$$

Como $V$ é gerado por vetores da forma acima, temos que $V$ admite decomposição em espaços de peso e seus pesos estão contidos em $D(\Lambda)=\left\{\Lambda-\alpha \mid \alpha \in Q_{+}\right\}$. Provando (i). 
Note que $V_{\Lambda}=U\left(\mathfrak{g}_{-}\right)_{0} v_{\Lambda}=\mathbb{k} v_{\Lambda}$. Por outro lado, para todo $\alpha \in Q_{+}$, existe uma quantidade finita de inteiros não negativos $k_{\beta}, \beta \in \Phi_{+}$, tais que $\alpha=\sum_{\beta \in \Phi_{+}} k_{\beta} \beta$. Como $V_{\Lambda-\alpha}$ é gerado por vetores da forma $\prod_{\beta \in \Phi_{+}} x_{-\beta}^{k_{\beta}} v_{\Lambda}$, temos que $V_{\Lambda-\alpha}$ possui dimensão finita. Finalizando a prova de (ii).

Se $v$ é um vetor de peso $\Lambda^{\prime}$ singular em $V$ tal que $v \notin \mathbb{k} v_{\Lambda}$, temos que $U(\mathfrak{g}) v$ é submódulo de $V$ de peso máximo $\Lambda^{\prime}$ e seus pesos são elementos de $D\left(\Lambda^{\prime}\right)$. Se $\Lambda^{\prime} \neq \Lambda$, então $\Lambda$ não é um peso de $U(\mathfrak{g}) v$, implicando que $U(\mathfrak{g}) v$ é um submoódulo próprio não nulo de $V$. Por outro lado, suponha que $\Lambda$ é único peso singular de $V$ e seja $W$ submódulo de $V$, então, pelo Lema 1.3.25, $W$ admite decomposição em espaços de pesos e

$$
W=\bigoplus_{\lambda \in D(\Lambda)} W \cap V_{\lambda}
$$

Seja $\lambda \in D(\Lambda)$ tal que $W \cap V_{\lambda} \neq 0$ e escreva $\lambda=\Lambda-\sum_{\alpha \in \Delta} a_{\alpha} k_{\alpha}$, onde $k_{\alpha} \in \mathbb{Z}_{+}$. Seja $v \in W \cap V_{\lambda}$ não nulo e suponha que $\sum_{\alpha \in \Delta} a_{\alpha}$ seja minimal, isto é, se $\lambda^{\prime}=\Lambda-\sum_{\alpha \in \Delta} b_{\alpha} \alpha$ é tal que $W \cap V_{\lambda^{\prime}} \neq 0$, então $\sum_{\alpha \in \Delta} a_{\alpha} \leq \sum_{\alpha \in \Delta} b_{\alpha}$. Então, para todo $\alpha \in \Phi_{+}, x_{\alpha} v \in V_{\lambda+\alpha}$ tem peso maior que $\lambda$, logo $E_{\alpha} v=0$. Sendo assim, $v \in V$ é singular e $\lambda=\Lambda$. Implicando que $V=U(\mathfrak{g}) v \subset W$. Logo, $W=V$ e $V$ irredutível, terminando a prova de (iii).

Por fim, basta notar que a soma de todos os submódulos próprios de $V$ satisfaz (i) e não contém $v_{\Lambda}$. Logo, este é o único submódulo maximal de $V$, provando (iv).

\subsubsection{Módulos de Verma}

Definição 1.4.6. Seja $\Lambda \in \mathfrak{h}^{*}$. Um g-módulo de peso máximo $M(\Lambda)$ e vetor de peso máximo $v_{\Lambda}$ é chamado de módulo de Verma (associado a $\Lambda$ ) se, para todo g-módulo de peso máximo $\Lambda$ com vetor de peso máximo $v$, existe um morfismo de $\mathfrak{g}$-módulos $\eta: M(\Lambda) \rightarrow M$ tal que $\eta\left(v_{\Lambda}\right)=v$.

Um módulo de Verma é um objeto universal no sentido que todo módulo de peso máximo é imagem quociente dele. Isso se deve ao fato de que o homomorfismo $\eta$ na definição é único, pois se $M(\Lambda)$ e $M$ são como acima, então $\eta\left(u \cdot v_{\Lambda} v_{+}\right)=u \cdot \eta\left(v_{\Lambda}\right)=u \cdot v$, para todo $u \in U\left(\mathfrak{g}_{-}\right)$. Sendo assim, como $M=U\left(\mathfrak{g}_{-}\right) v$, temos que $\eta$ é sobrejetor e é totalmente determinado por $\eta\left(v_{\Lambda}\right)$. Note que a escolha de um vetor de peso máximo em $M$ determina um homomorfismo $\eta$ diferente.

Proposição 1.4.7. Seja $\Lambda \in \mathfrak{h}^{*}$ e suponha que existe um módulo de Verma $M(\Lambda)$, então todo módulo de Verma associado a $\Lambda$ é isomorfo a $M(\Lambda)$, como $\mathfrak{g}$-módulos.

Demonstração. Se $M(\Lambda)^{\prime}$ é outro módulo de Verma com vetor de peso máximo $v_{\Lambda}^{\prime}$, então existe um único homomorfismo de $\mathfrak{g}$-módulos $\eta: M(\Lambda) \rightarrow M\left(\Lambda^{\prime}\right)$ tal que $\eta\left(v_{\Lambda}\right)=v_{\Lambda}^{\prime}$. Por outro lado, existe um único homomorfismo de $\mathfrak{g}$-módulos $\eta^{\prime}: M(\Lambda)^{\prime} \rightarrow M(\Lambda)$ tal que $\eta\left(v_{\Lambda}^{\prime}\right)=v_{\Lambda}$. Pelo argumento dado acima, $\eta \circ \eta^{\prime}=i d_{M(\Lambda)^{\prime}}$ e $\eta \circ \eta^{\prime}=i d_{M(\Lambda)}$. Logo, $M(\Lambda)$ e $M(\Lambda)^{\prime}$ são isomorfos.

Proposição 1.4.8. Seja $\Lambda \in \mathfrak{h}^{*}$, então existe um módulo de Verma $M(\Lambda)$.

Demonstração. Seja $\mathfrak{b}=\mathfrak{h} \oplus \mathfrak{g}_{+}$, então $\mathfrak{b}$ é um subálgebra de Lie de $\mathfrak{g}$ e podemos ver $U(\mathfrak{b})$ como uma subálgebra de $U(\mathfrak{g})$. Logo, $U(\mathfrak{g})$ é um módulo à esquerda de $U(\mathfrak{b})$ quando visto como subanel de $U(\mathfrak{g})$.

Seja $\mathbb{k} v_{\Lambda}$ um espaço vetorial de dimensão 1 gerado por $v_{\Lambda}$. Defina em $\mathbb{k} v_{\Lambda}$ uma estrutura de $\mathfrak{b}$-módulo por

$$
\begin{gathered}
\mathfrak{g}_{+} \cdot v_{\Lambda}=0, \\
h \cdot v_{\Lambda}=\Lambda(h) v_{\Lambda}, \text { para todo } h \in \mathfrak{h} .
\end{gathered}
$$

Sendo assim, consideraremos o módulo induzido

$$
M(\Lambda):=U(\mathfrak{g}) \otimes_{U(\mathfrak{b})} \mathbb{k} v_{\Lambda}
$$


Temos que $M(\Lambda)$ é um $U(\mathfrak{g})$-módulo à esquerda e, portanto, um $\mathfrak{g}$-módulo. A ação de $x \in \mathfrak{g}$ em $u \otimes v \in M(\Lambda)$ é dada por $x \cdot(u \otimes v)=(x u) \otimes v$. Além disso, para todo $x \in U(\mathfrak{b}) \subset U(\mathfrak{g}) \mathrm{e}$ $u \otimes v \in M(\Lambda)$, temos que $x \cdot(u \otimes v)=u \otimes x v+[x, u] \otimes v$. Em particular, se considerarmos $1 \otimes v_{\Lambda} \in M(\Lambda)$, então, para todo $x \in \mathfrak{g}_{+}$,

$$
x \cdot\left(1 \otimes v_{\Lambda}\right)=1 \otimes\left(x \cdot v_{\Lambda}\right)=0,
$$

e, para todo $h \in \mathfrak{h}$,

$$
h \cdot\left(1 \otimes v_{\Lambda}\right)=1 \otimes\left(h \cdot v_{\Lambda}\right)=1 \otimes \Lambda(h) v_{\Lambda} .
$$

É fácil ver que $U(\mathfrak{g})\left(1 \otimes v_{\Lambda}\right)=U(\mathfrak{g}) \otimes_{U(\mathfrak{b})} \mathbb{k} v_{\Lambda}=M(\Lambda)$. Logo, $M(\Lambda)$ é um $\mathfrak{g}$-módulo de peso máximo $\Lambda$ e vetor de peso máximo $1 \otimes v_{\Lambda}$.

Seja $M$ qualquer módulo de peso máximo $\Lambda$ e vetor de peso máximo $v$. Seja $\varphi: U(\mathfrak{g}) \times \mathbb{k} v_{\Lambda} \rightarrow M$ dada por $\phi\left(u, a v_{\Lambda}\right)=(a u) \cdot v$. Portanto, $\varphi$ é uma aplicação bilinear e para todo $b \in U(\mathfrak{b})$,

$$
\varphi\left(u w, a v_{+}\right)=(a u w) \cdot v=(a u) \cdot(w \cdot v)=\varphi(u, w \cdot a v) .
$$

Sendo assim, existe uma transformação linear $\hat{\varphi}: U(\mathfrak{g}) \otimes_{U(\mathfrak{b})} \mathbb{k} v_{\Lambda} \rightarrow M$ tal que $\hat{\varphi}\left(u \otimes a v_{\Lambda}\right)=a u \cdot v$. Logo, $\hat{\varphi}\left(1 \otimes v_{\Lambda}\right)=v$ e $\hat{\varphi}$ é morfismo de $U(\mathfrak{g})$-módulos.

Definição 1.4.9. Dizemos que um $\mathfrak{g}$-módulo $M$ é $U\left(\mathfrak{g}_{-}\right)$-livre de torsão se para $u \in U\left(\mathfrak{g}_{-}\right)$e $m \in M$, temos que

$$
u \cdot m=0 \Longrightarrow u=0 \text { ou } m=0
$$

Proposição 1.4.10. Seja $M$ um g-módulo de peso máximo $\Lambda$ com vetor de peso máximo v. Se $M$ é $U\left(\mathfrak{g}_{-}\right)$-livre de torsão, então $M$ é um módulo de Verma.

Demonstração. Temos que existe um morfismo sobrejetor de $\mathfrak{g}$-módulos $\eta: M(\Lambda) \rightarrow M$ tal que $\eta\left(v_{\Lambda}\right)=v$. Basta mostrar que se para $u \in U\left(\mathfrak{g}_{-}\right)$e $m \in M$, temos que

$$
u \cdot m=0 \Longrightarrow u=0 \text { ou } m=0
$$

então $\eta$ é injetor. Temos que todo elemento em $M$ é da forma $u \cdot v$, para algum $u \in U\left(\mathfrak{g}_{-}\right)$. Seja $u \in U\left(\mathfrak{g}_{-}\right)$tal que $u \cdot v_{\Lambda}$ está no núcleo de $\eta$. Então, $0=\eta\left(u \cdot v_{\Lambda}\right)=u \cdot v$. Como $v \neq 0$, segue que $u=0$. Logo, $\eta$ é um isormofismo e, sendo assim, $M$ é módulo de Verma.

Corolário 1.4.11. Qualquer submódulo de peso máximo de um módulo de Verma é um módulo de Verma.

Demonstração. Pela proposição anterior, $M(\Lambda)$ é $U\left(\mathfrak{g}_{-}\right)$-livre de torsão. Além disso, é facil ver que todo submódulo de $M \subset M(\Lambda)$ também será $U\left(\mathfrak{g}_{-}\right)$-livre de torsão. Em particular, se $M$ é um submódulo de peso máximo, então $M$ é $U\left(\mathfrak{g}_{-}\right)$-livre de torsão e, pela proposição anterior, é um módulo de Verma.

Corolário 1.4.12. Se $\Lambda \in \mathfrak{h}^{*}$, então $\Pi(M(\Lambda))=D(\Lambda)$.

Proposição 1.4.13. Seja $\Lambda \in \mathfrak{h}^{*}$.

(i) Se $M(\Lambda)$ é o módulo de Verma com peso máximo $\Lambda$, então $M(\Lambda)$ possui um único ideal maximal próprio $N(\Lambda)$. Em particular, $L(\Lambda):=M(\Lambda) / N(\Lambda)$ é irredutivel e é o único $\mathfrak{g}$ módulo de peso máximo irredutivel com péso máximo $\Lambda$.

(ii) Se $M$ é um $\mathfrak{g}$-módulo peso máximo $\Lambda$ e $N$ é seu único submódulo próprio maximal, então $M / N$ e $L(\Lambda)$ são isomorfos.

Demonstração. $M(\Lambda)$ é um módulo de peso máximo $\Lambda$, então, pela Proposição 1.4.1, todos os pesos de $M(\Lambda)$ estão em $D(\Lambda)$ e $M(\Lambda)$ possui um único submódulo maximal $N(\Lambda)$. 
$L(\Lambda)=M(\Lambda) / N(\Lambda)$ é um módulo de peso máximo com vetor de peso máximo $v_{\Lambda}+N(\Lambda)$ e peso máximo $\Lambda . L(\Lambda)$ é irredutível, pois $N(\Lambda)$ é submódulo maximal de $M(\Lambda)$. Se $L$ é um módulo de peso máximo $\Lambda$ irredutível e vetor de peso máximo $v$, então existe um morfismo de $\mathfrak{g}$-módulos $\eta: M(\Lambda) \rightarrow L \operatorname{com} \eta\left(v_{\Lambda}\right)=v$. Como $\eta$ é sobrejetor e $L$ é irredutível, temos que o núcleo de $\eta$ é um submódulo maximal próprio de $M(\Lambda)$. Portanto, o núcleo de $\eta$ é $N(\Lambda)$ e

$$
M(\Lambda) / N(\Lambda) \cong L
$$

Sendo assim, provamos (i).

Se $M$ é um $\mathfrak{g}$-módulo de peso máximo $\Lambda$, então existe um morfismo de $\mathfrak{g}$-módulos sobrejetor $\eta^{\prime}: M(\Lambda) \rightarrow M$. Todo submódulo maximal de $M$ será imagem de um submódulo maximal de $M(\Lambda)$ por $\eta^{\prime}$. Como o único submódulo maximal de $M(\Lambda)$ é $N(\Lambda)$, temos que o único submódulo maximal de $M$ é $N=\eta^{\prime}(N(\Lambda))$. Logo,

$$
M / N \cong\left(M(\Lambda) / \operatorname{Ker}\left(\eta^{\prime}\right)\right) /\left(N(\Lambda) / \operatorname{Ker}\left(\eta^{\prime}\right)\right) \cong M(\Lambda) / N(\Lambda)=L(\Lambda)
$$

Pela Proposição 1.4.3, o conjunto de todos os g-módulo de dimensão finita simples está contido em $\left\{L(\Lambda) \mid \Lambda \in \mathfrak{h}^{*}\right\}$. Portanto, dado $\Lambda \in \mathfrak{h} *$, falta determinar quando $L(\Lambda)$ possui dimensão finita. O próximo teorema que iremos enunciar, dá exatamente um critério para isso.

Teorema 1.4.14. Seja $\Lambda \in \mathfrak{h}^{*}$, então $L(\Lambda)$ possui dimensão finita se e somente $\Lambda(\alpha) \in \mathbb{Z}_{+}$para todo $\alpha \in \Delta$.

Demonstração. Veja [Hum78], Theorem 21.1 e Theorem 21.2.

Com este teorema, a Proposição 1.4.3 e o Teorema de Weyl, temos que se $V$ é um g-módulo de dimensão finita, então existem $\Lambda_{1}, \ldots, \Lambda_{r} \in \mathfrak{h}^{*}$ tais que $\Lambda_{i}(\alpha) \in \mathbb{Z}_{+}$, para todo $i=1, \ldots, r$ e $\alpha \in \Delta$, e

$$
V \cong L\left(\Lambda_{1}\right) \oplus \ldots \cdots \oplus L\left(\Lambda_{r}\right)
$$

\subsection{Variedades Algébricas e Teorema de Kostant}

Um famoso teorema de Kostant (veja [Kos63, Theorem 21]) diz que a álgebra envolvente de uma álgebra de Lie complexa redutível é livre como um módulo sobre seu centro. Nesta seção, resumiremos a técnica que utilizaremos para provar uma versão do Teorema de Kostant para algumas álgebras correntes, além de fixar notações, definições e resultados que usaremos principalmente no Capítulo 3. Utilizaremos resultados que muitas vezes são encontrados em livros sobre álgebra comutativa, recomendamos [AM69], [Eis95], [Mat87] e [ZS76]. Para uma referência em português, veja [BT15].

Lembre que $\mathbb{k}$ é um corpo algebricamente fechado de característica 0.

Definição 1.5.1. (i) O espaço afim $\mathbb{A}_{\mathbb{k}}^{n}$ de dimensão $n$ sobre o corpo $\mathbb{k}$ é o conjunto $\mathbb{A}_{\mathbb{k}}^{n}=\mathbb{k}^{n}$.

(ii) Seja $S \subset \mathbb{k}\left[x_{1}, \ldots, x_{n}\right]$ um conjunto de polinômios em $n$ variáveis. O conjunto algébrico afim associado ao conjunto $S$ é o subconjunto de $\mathbb{A}_{\mathfrak{k}}^{n}$ definido por

$$
V(S)=\left\{\left(a_{1}, \ldots, a_{n}\right) \in \mathbb{A}_{\mathbb{k}}^{n} \mid f\left(a_{1}, \ldots, a_{n}\right)=0 \text { para todo } f \in S\right\} .
$$

(iii) Dizemos que $X \subset \mathbb{A}_{\mathbb{k}}^{n}$ é um conjunto algébrico se existir $S \subset \mathbb{k}\left[x_{1}, \ldots, x_{n}\right]$ tal que $X=V(S)$.

Se $S \subset \mathbb{k}\left[x_{1}, \ldots, x_{n}\right]$, denotaremos por $\langle S\rangle$ o ideal de $\mathbb{k}\left[x_{1}, \ldots, x_{n}\right]$ gerado por $S$. Note que se $I \subset \mathbb{k}\left[x_{1}, \ldots, x_{n}\right]$ é tal que $I=\langle S\rangle$, então $V(I)=V(\langle S\rangle)=V(S)$. Portanto, poderíamos ter definido conjunto algébricos como o conjunto de zeros de um ideal. Uma consequência do seguinte teorema é que todo conjunto algébrico é o conjunto de zeros de um número finito de polinômios. 
Teorema 1.5.2 (Teorema da base de Hilbert). Se $I \subset \mathbb{k}\left[x_{1}, \ldots, x_{n}\right]$, então I é finitamente gerado. Isto é, existem $f_{1}, \ldots, f_{k}$ tais que $I=\left\langle f_{1}, \ldots, f_{k}\right\rangle$.

Demonstração. Este é um resultado clássico e pode ser encontrado em diversos livros sobre álgebra comutativa. Por exemplo, veja Theorem 1.2 de [Eis95].

Quando $S=\left\{f_{1}, \ldots, f_{k}\right\}$ for um conjunto finito, denotaremos $V(S)=V\left(\left\{f_{1}, \ldots, f_{k}\right\}\right)=$ $V\left(f_{1}, \ldots, f_{k}\right)$.

Exemplo 1.5.3. 1. O espaço afim $\mathbb{A}_{\mathbb{k}}^{n}$ é um conjunto algébrico, pois $\mathbb{A}_{\mathbb{k}}^{n}=V(0)$. O conjunto vazio $\emptyset$ é um conjunto algébrico afim, pois $\emptyset=V\left(\mathbb{k}\left[x_{1}, \ldots, x_{n}\right]\right)$.

2. Se $\left(a_{1}, \ldots, a_{n}\right) \in \mathbb{A}_{k}^{n}$, então $\left\{\left(a_{1}, \ldots, a_{n}\right)\right\}$ é um conjunto algébrico. Basta ver que $V\left(x_{1}-\right.$ $\left.a_{1}, \ldots, x_{n}-a_{n}\right)=\left\{\left(a_{1}, \ldots, a_{n}\right)\right\}$.

Proposição 1.5.4 (Topologia de Zariski). As seguintes propriedades são verdadeiras:

(i) $\mathbb{A}_{\mathbb{k}}^{n}=V(0)$ e $\emptyset=V\left(\mathbb{k}\left[x_{1}, \ldots, x_{n}\right]\right)$.

(ii) Se $I, J$ são ideais de $\mathbb{k}\left[x_{1}, \ldots, x_{n}\right]$, então $V(I) \cup V(J)=V(I J)$. Em particular, se $f, g \in$ $\mathbb{k}\left[x_{1}, \ldots, x_{n}\right]$, então $V(f g)=V(f) \cup V(g)$.

(iii) $S e\left\{I_{j}\right\}_{j \in S}$ é uma família de ideais de $\mathbb{k}\left[x_{1}, \ldots, x_{n}\right]$, então $\bigcap_{j \in S} V\left(I_{j}\right)=V\left(\sum_{j \in S} I_{j}\right)$.

Portanto, os conjuntos algébricos formam os fechados de uma topologia em $\mathbb{A}_{\mathbb{k}}^{n}$.

Demonstração. A primeira propriedade é óbvia. Seja $\left(a_{1}, \ldots, a_{n}\right) \in V(I)$, então um elemento qualquer $\sum_{i=1}^{r} f_{i} g_{i}, f_{i} \in I, g_{i} \in J$, se anula em $\left(a_{1}, \ldots, a_{n}\right)$. O mesmo vale para $\left(a_{1}, \ldots, a_{n}\right) \in V(J)$, portanto $V(I) \cup V(J) \subset V(I J)$. Por outro lado, seja $\left(a_{1}, \ldots, a_{n}\right) \in V(I J)$ tal que $\left(a_{1}, \ldots, a_{n}\right) \notin$ $V(J)$, então existe $g \in J$ tal que $g\left(a_{1}, \ldots, a_{n}\right) \neq 0$. Temos que $f g$ se anula $V(I J)$, para todo $f \in I$. Logo, $f\left(a_{1}, \ldots, a_{n}\right) g\left(a_{1}, \ldots, a_{n}\right)=0$, para todo $f \in I$. Sendo assim, $\left(a_{1}, \ldots, a_{n}\right) \in V(I)$. Como o mesmo vale para $\left(a_{1}, \ldots, a_{n}\right) \notin V(I)$, temos que $V(I J) \subset V(I) \cup V(J)$.

Seja $\left\{I_{j}\right\}_{j \in S}$ uma família de ideais de $\mathbb{k}\left[x_{1}, \ldots, x_{n}\right]$, então $\left(a_{1}, \ldots, a_{n}\right) \in \bigcap_{j \in J} V\left(I_{j}\right)$ se, e somente se, $f\left(a_{1}, \ldots, a_{n}\right)=0$ para todo $f \in I_{j}, j \in S$, se, e somente se, $f\left(a_{1}, \ldots, a_{n}\right)=0$ para todo $f \in \sum_{j \in S} I_{j}$ se, e somente se, $\left(a_{1}, \ldots, a_{n}\right) \in V\left(\sum_{j \in S} I_{j}\right)$.

Seja $X$ um conjunto algébrico em $\mathbb{A}_{k}^{n}$, então a topologia de Zariski induz uma topologia em $X$. Ou seja, $Y \subset X$ é um conjunto fechado de $X$ se existe um conjunto algébrico afim $Y^{\prime} \subset \mathbb{A}_{\mathbb{k}}^{n}$ tal que $Y=Y^{\prime} \cap X$.

Definição 1.5.5. Seja $X \subset \mathbb{A}_{\mathbb{k}}^{n}$ um conjunto algébrico. O ideal associado a $X$ é o conjunto

$$
I(X)=\left\{f \in \mathbb{k}\left[x_{1}, \ldots, x_{n}\right] \mid f\left(a_{1}, \ldots, a_{n}\right)=0 \text { para todo }\left(a_{1}, \ldots, a_{n}\right) \in X\right\} .
$$

É possível mostrar que $I(X)$ é o núcleo de um homomorfismo de anéis, portanto $I(X)$ é um ideal de $\mathbb{k}\left[x_{1}, \ldots, x_{n}\right]$.

Teorema 1.5.6 (Teorema dos zeros de Hilbert, versão clássica). Seja a um ideal de $\mathbb{k}\left[x_{1}, \ldots, x_{n}\right]$, então os ideais de maximais de $\mathbb{k}\left[x_{1}, \ldots, x_{n}\right] / \mathfrak{a}$ são precisamente os ideais $\left\langle x_{1}-a_{1}, \ldots, x_{n}-a_{n}\right\rangle$ com $\left(a_{1}, \ldots, a_{n}\right) \in V(\mathfrak{a})$. Além disso, $V(\mathfrak{a})=\emptyset$ se, e somente se, $\mathfrak{a}=\mathbb{k}\left[x_{1}, \ldots, x_{n}\right]$. Por fim,

$$
I(V(\mathfrak{a}))=\sqrt{\mathfrak{a}}
$$

Demonstração. Veja Theorem 1.6 e Corollary 1.9 de [Eis95]. Para uma prova da versão mais forte e geral de tal teorema, veja Theorem 4.19 do mesmo livro. 
Sendo assim, $I(X)$ é um ideal radical de $\mathbb{k}\left[x_{1}, \ldots, x_{n}\right]$ se $X \subset \mathbb{A}_{k}^{n}$ é um conjunto algébrico. Além disso, os ideais maximais de $\mathbb{k}\left[x_{1}, \ldots, x_{n}\right]$ são exatamente os elementos do conjunto

$$
\left\{\left(x_{1}-a_{1}, \ldots, x_{n}-a_{n}\right) \mid\left(a_{1}, \ldots, a_{n}\right) \in \mathbb{A}_{\mathbb{k}}^{n}\right\}
$$

Definição 1.5.7. Uma conjunto algébrico afim $X$ é dito irredutível, se não existem conjuntos algébricos $A, B \subset X$ próprios não-vazios tais que $X=A \cup B$. Se $X$ é um conjunto algébrico irredutível, dizemos que $X$ é uma variedade algébrica.

Se $X$ é um conjunto algébrico e $Y \subset \mathbb{A}_{\mathbb{k}}^{n}$ é uma variedade algébrica, dizemos que $X \cap Y$ é uma subvariedade algébrica de $X$.

Proposição 1.5.8. Seja $X \subset \mathbb{A}_{k \mathrm{k}}^{n}$ um conjunto algébrico afim, então $X$ é uma variedade algébrica se, e somente se, $I(X)$ é um ideal primo.

Demonstração. Suponha que $X$ é irredutível. Sejam $f, g \in \mathbb{A}_{\mathfrak{k}}^{n}$ tais que $f g \in I$, mas $f, g \notin I(X)$. Sendo assim, $X \cup V(f) \cup V(g)=V(f g)$ se, e somente se, $X=(X \cap V(f)) \cup(X \cap V(g))$. Como $f, g \not h I$, temos que $V(f) \cap X$ e $V(g) \cap X$ são subconjuntos algébricos próprios não-vazios de $X$. $\mathrm{O}$ que é uma contradição com a hipótese de que $X$ é irredutível. Logo, $I(X)$ é primo.

Por outro lado, suponha que $I(X)$ é primo. Se existem conjuntos algébricos próprios não-vazios $A, B$ tais que $X=A \cup B$, então existem $f, g \in \mathbb{k}\left[x_{1}, \ldots, x_{n}\right]$ tais que $f \in I(A) \backslash I(B)$ e $g \in I(B) \backslash I(A)$. Porém, $f g \in I(A) I(B)$ se anula em identicamente em $X$, implicando que $f g \in I(X)$ e $f, g \notin I(X)$. O que é uma contradição com a hipótese de que $I(X)$ é primo. Logo, $X$ é irredutível.

Podemos ver $V$ e $I$ como aplicações nos ideais de $\mathbb{k}\left[x_{1}, \ldots, x_{n}\right]$ e conjuntos algébricos em $\mathbb{A}_{\mathbb{k}}^{n}$, respectivamente. O Teorema de Zeros de Hilbert diz estas aplicações induzem uma correspondência biunívoca entre os subconjuntos algébricos de $\mathbb{A}_{\mathbb{k}}^{n}$ e os ideais radicais de $\mathbb{k}\left[x_{1}, \ldots, x_{n}\right]$ :

$$
\left.\left\{\text { Conjuntos algébricos afins em } \mathbb{A}_{\mathbb{k}}^{n}\right\} \stackrel{I}{\longleftarrow} \text { 价eais radicais de } \mathbb{k}\left[x_{1}, \ldots, x_{n}\right]\right\}
$$

Ademais, definem uma correspondência biunívoca entre os ideais primos de $\mathbb{k}\left[x_{1}, \ldots, x_{n}\right]$ e variedades algébricas em $\mathbb{A}_{\mathbb{k}}^{n}$. Por fim, também definem uma correspondência biunívoca entre os ideais maximais de $\mathbb{k}\left[x_{1}, \ldots, x_{n}\right]$ e pontos de $\mathbb{A}_{\mathbb{k}}^{n}$.

Definição 1.5.9. Seja $X \subset \mathbb{A}_{\mathbb{k}}^{n}$ um conjunto algébrico afim. $\mathrm{O}$ anel de coordenadas associado a $X$ é

$$
\mathbb{k}[X]=\mathbb{k}\left[x_{1}, \ldots, x_{n}\right] / I(X) .
$$

Exemplo 1.5.10. Como $I\left(\mathbb{A}_{\mathbb{k}}^{n}\right)$ é o ideal nulo, temos que $\mathbb{k}\left[\mathbb{A}_{\mathbb{k}}^{n}\right]=\mathbb{k}\left[x_{1}, \ldots, x_{n}\right]$.

Como $\mathbb{k}\left[x_{1}, \ldots, x_{n}\right]$ é finitamente gerada, todo anel de coordenadas é finitamente gerado. Além disso, como $I(X)$ é radical, temos que $\mathbb{k}[X]$ é reduzido, para todo conjunto algébrico afim $X$. Pelo Teorema da Correspondência, existe uma correspondência biunívoca entre os ideais de $\mathbb{k}[X]$ e os ideais de $\mathbb{k}\left[x_{1}, \ldots, x_{n}\right]$ que contém $I(X)$. Além disso, esta correspondência preserva as correspondências induzidas por $I$ e $V$ entre os conjuntos algébricos afins em $X$ e ideais radicais de $\mathbb{k}[X]$.

Definição 1.5.11. Seja $X \subset \mathbb{A}_{\mathbb{k}}^{n}$ e $Y=\mathbb{A}_{\mathbb{k}}^{m}$ dois conjuntos algébricos afins. Dizemos que $f: X \rightarrow Y$ é um morfismo de conjuntos algébricos se $f$ é restrição de uma aplicação polinomial de $\mathbb{A}_{\mathbb{k}}^{n}$ para $\mathbb{A}_{\mathbb{k}}^{m}$. Ou seja, existem polinômios $f_{1}, \ldots, f_{m} \in \mathbb{k}\left[x_{1}, \ldots, x_{n}\right]$ tais que

$$
f\left(a_{1}, \ldots, a_{n}\right)=\left(f_{1}\left(a_{1}, \ldots, a_{n}\right), \ldots, f_{m}\left(a_{1}, \ldots, a_{n}\right)\right)
$$

para todo $\left(a_{1}, \ldots, a_{n}\right) \in X$. 
Exemplo 1.5.12. A álgebra $\mathbb{k}[X]$ é isomorfa a uma $\mathbb{k}$-álgebra definida no conjunto de todos os morfismos de conjuntos algébricos $X \rightarrow \mathbb{A}^{1}$.

Exemplo 1.5.13. Se $f: X \rightarrow Y$ é um morfismos entre dois conjuntos algébricos afins $X \subset \mathbb{A}_{k}^{n}$ e $Y=\mathbb{A}_{\mathbb{k}}^{m}$, então a aplicação linear

$$
\begin{aligned}
& f^{*}: \mathbb{k}[X] \rightarrow \mathbb{k}[Y] \\
& \psi \rightarrow \psi \circ f
\end{aligned}
$$

é um homomorfismo de k-álgebras, chamado de pullback de $f$.

Utilizaremos a definição de dimensão de Krull dada na Definição 1.1.18 para definir a dimensão de um conjunto algébrico afim.

Definição 1.5.14. Seja $X$ um conjunto algébrico afim. Definimos a dimensão de $X$ como sendo a dimensão de Krull de $\mathbb{k}[X]$.

Como a dimensão de Krull de $\mathbb{k}\left[x_{1}, \ldots, x_{n}\right]$ é $n$, então a dimensão de $\mathbb{A}_{\mathbb{k}}^{n}$ é $n$. Existem outras noções de dimensão de um conjunto algébrico. Uma delas é o supremo do comprimento das cadeias estritamente crescentes de subconjuntos fechados irredutíveis de um conjunto algébrico afim $X$, que é o mesmo valor da dimensão de Krull no anel de coordenadas $\mathbb{k}[X]$, por isso definimos a dimensão de $X$ da forma acima.

Proposição 1.5.15. Seja $X \subset \mathbb{A}_{\mathbb{k}}^{n}$ um conjunto algébrico, então existem únicas $X_{1}, \ldots, X_{k} \subset \mathbb{A}_{k}^{n}$ variedades algébricas tais que

$$
X=X_{1} \cup X_{2} \cup \cdots \cup X_{k}
$$

e $X_{i} \not \subset X_{j}, i \neq j$. Neste caso, diremos que cada $X_{i}$ é uma componente irredutivel de $X$.

Demonstração. Veja Theorem 13 na página 162 de [ZS76].

Definição 1.5.16. Seja $X$ um conjunto algébrico afim. Dizemos que $X$ é equidimensional, se a dimensão de suas componentes irredutíveis forem iguais. Se $X$ é equidimensional, dizemos que é equidimensional de dimensão $k$, se a dimensão de uma de suas componentes irredutíveis for igual a $k$.

Exemplo 1.5.17. Considere o conjunto algébrico $V(x y, x+y) \subset \mathbb{A}_{\mathbb{k}}^{2}$, então

$$
V(x y)=V(x) \cup V(y) .
$$

Portanto, $V(x y)$ é equidimensional de dimensão 1.

Exemplo 1.5.18. Considere o conjunto algébrico $V(x y, x z) \subset \mathbb{A}_{\mathbb{k}}^{2}$, então

$$
V(x y, x z)=V(x) \cup V(y, x) \cup V(y, z) .
$$

Como $V(x)$ é uma variedade algébrica de dimensão 2 e $V(y, z)$ é uma variedade algébrica de dimensão 1 , então $V(x y, y z)$ não é equidimensional.

Definição 1.5.19. Seja $\Lambda$ uma álgebra comutativa associativa com unidade e finitamente gerada. Dizemos que $g_{1}, \ldots, g_{r} \in \Lambda$ é uma sequência regular, se $g_{1}$ não é divisor de zero e não é inversível em $\Lambda$ e a imagem de $g_{i}$ não é um divisor de zero e não é invertível em $\Lambda /\left\langle g_{1}, \ldots, g_{i-1}\right\rangle$, para cada $i=2, \ldots, r$. Dizemos que $\Lambda$ é uma álgebra intersecção completa, se $\Lambda$ é reduzida e existir uma sequência regular em $g_{1}, \ldots, g_{t} \in \mathbb{k}\left[x_{1}, \ldots, x_{n}\right]$ tal que $\Lambda=\mathbb{k}\left[x_{1}, \ldots, x_{n}\right] /\left\langle g_{1}, \ldots, g_{t}\right\rangle$.

Proposição 1.5.20. Seja $\Lambda$ uma álgebra interseção completa graduada com dimensão de Krull $n$ e seja $\mathbf{g}=\left\{g_{1}, \ldots, g_{t}\right\}$ uma sequência que consiste de elementos homogêneos de $\Lambda, 1 \leq t \leq n$, então 
1. Suponha que $\mathbf{g}$ é regular. Então $g_{\sigma(1)}, \ldots, g_{\sigma(t)}$ é uma sequência regular para toda permutação $\sigma$ de $\{1, \ldots, n\}$. Em particular, qualquer subsequência de $\mathbf{g}$ é regular.

2. g é uma sequência regular em $\Lambda$ se, e somente se, a variedade $V\left(g_{1}, \ldots, g_{t}\right)$ é equidimensional de dimensão $n-t$.

Demonstração. Para a prova do primeiro item, veja [Mat87, p. 127]. Para a prova do segundo item, veja [FO05, Proposition 2.1].

Observação 1.5.21. Se $g_{1}, \ldots, g_{t} \in \mathbb{k}\left[x_{1}, \ldots, x_{n}\right]$ é uma sequência regular de elementos homogêneos e

$$
\Lambda=\mathbb{k}\left[x_{1}, \ldots, x_{n}\right] /\left\langle g_{1}, \ldots, g_{t}\right\rangle
$$

é uma álgebra intersecção completa, então $\Lambda=\mathbb{k}[X]$, onde $X=V\left(g_{1}, \ldots, g_{t}\right)$. Pela Proposição 1.5.20, $V\left(g_{1}, \ldots, g_{i}\right)$ tem dimensão $n-i$, para cada $i=1, \ldots, t$. Isso implica que, para obter $X$ como intersecção de $V\left(g_{i}\right), i=1, \ldots, t$, é necessário intersectar todos eles e, por isso, o nome intersecção completa.

Observação 1.5.22. Todas as sequências que consideraremos serão polinômios homogêneos. Pela Proposição anterior, não precisaremos especificar uma ordem para tratar das sequências.

Exemplo 1.5.23. Se $X=V\left(g_{1}, \ldots, g_{t}\right) \subset \mathbb{A}_{\mathbb{k}}^{n}$ é irredutível e possui dimensão $n-t$, então $\Lambda=\mathbb{k}[X]$ é uma álgebra intersecção completa.

Lema 1.5.24. Seja $A=\mathbb{k}\left[x_{1}, \ldots, x_{n}\right]$ a álgebra polinomial em $n$ variáveis e sejam $G_{1}, \ldots, G_{t} \in A$. A sequência

$$
x_{1}, \ldots, x_{r}, G_{1}, \ldots, G_{t}
$$

é regular em $A$ se, e somente se, $g_{1}, \ldots, g_{t}$ é uma sequência regular em $\mathbb{k}\left[x_{r+1}, \ldots, x_{n}\right]$, onde

$$
g_{i}\left(x_{r+1}, \ldots, x_{n}\right)=G\left(0, \ldots, 0, x_{r+1}, \ldots, x_{n}\right)
$$

para cada $i=1, \ldots, t$.

Demonstração. Veja [FO05, Lemma 2.2].

Tendo em vista o Lema 1.5.24, fixaremos a notação a seguir para facilitar a dissertação das demonstrações de resultados futuros.

Notação 1.5.25. Seja $P \in \mathbb{C}\left[x_{1}, \ldots, x_{n}\right]$ um polinômio em $n$ variáveis e $\mathbf{X}=\left\{x_{i_{1}}, \ldots, x_{i_{r}}\right\}$ um conjunto de variáveis. Denotaremos por $P^{\mathbf{X}}$ o polinômio obtido de $P$ substituindo $x_{i_{1}}=\cdots=$ $x_{i_{r}}=0$. Note que $P^{\mathbf{X}}$ pode ser visto como um elemento de $\mathbb{C}\left[x_{1}, \ldots, x_{n}\right]$ ou $\mathbb{C}\left[\left\{x_{1}, \ldots, x_{n}\right\} \backslash \mathbf{X}\right]$.

Para mostrarmos como a Proposição 1.5.20 e Lema 1.5.24 funcionam juntos, iremos provar o seguinte resultado.

Proposição 1.5.26. Considere $\mathbb{k}\left[x_{1}, \ldots, x_{n}\right]$ a álgebra polinomial em $n$ variáveis, então os polinômios simétricos em $n$ variáveis formam uma sequência regular. Isto é, a sequência $g_{n 1}, \ldots, g_{n n}$ é regular, onde

$$
g_{n t}=\sum_{i_{1}<\cdots<i_{t}} x_{i_{1}} \ldots x_{i_{t}}, \quad t \in\{1, \ldots, n\} .
$$

Demonstração. Iremos provar por indução em $n$. Se $n=1$, então $g_{11}=x_{1}$ não é inversível nem divisor de zero. Portanto, é regular. Suponha $n>1$ e que, por indução, $g_{n-1,1}, \ldots, g_{n-1, n-1}$ é regular. Portanto, $V\left(g_{n-1,1}, \ldots, g_{n-1, n-1}\right)$ é equidimensional de dimensão 0. Sendo assim, basta mostrar que $V\left(g_{n n}, g_{n, n-1}, \ldots, g_{n 1}\right)$ é equidimensional de dimensão 0 também. Temos que

$$
\begin{aligned}
V\left(g_{n n}, g_{n, n-1}, \ldots, g_{n 1}\right) & =V\left(x_{1} \ldots x_{n}, g_{n, n-1}, \ldots, g_{n 1}\right) \\
& =V\left(x_{1}, g_{n, n-1}, \ldots, g_{n 1}\right) \cup \cdots \cup V\left(x_{n}, g_{n, n-1}, \ldots, g_{n 1}\right) .
\end{aligned}
$$


Logo, basta mostrar que

$$
V\left(x_{i}, g_{n, n-1}, \ldots, g_{n 1}\right)
$$

é equidimensional de dimensão 0 , para cada $i=1, \ldots, n$. Seja $\mathbf{X}_{\mathbf{i}}=\left\{x_{i}\right\}$, então se fizermos a mudança de variável

$$
x_{j} \longleftrightarrow \begin{cases}x_{j} & \text { se } j=1, \ldots, i-1 \\ x_{j-1} & \text { se } i=i+1, \ldots, n\end{cases}
$$

em $g_{n, t}^{\mathbf{X}_{\mathbf{i}}}$ teremos exatamente $g_{n-1, t}$, para cada $i=1, \ldots, n$ e $t=1, \ldots, n-1$. Portanto, pela hipótese de indução e pelo Lema 1.5.24, $x_{i}, g_{n, n-1}, \ldots, g_{n 1}$ é regular. Assim, pela Proposição 1.5.20, $V\left(x_{i}, g_{n, n-1}, \ldots, g_{n 1}\right)$ é equidimensional de dimensão 0 , para cada $i=1, \ldots, n$. Logo, pela mesma proposição, $g_{n 1}, \ldots, g_{n n}$ é regular.

Definição 1.5.27. Seja $U$ uma álgebra associativa com unidade filtrada. Se $\bar{U}$ é uma álgebra interseç̧ão completa, dizemos que $U$ é filtrada especial.

Exemplo 1.5.28. Seja $\mathfrak{g}$ uma álgebra de Lie de dimensão finita $n$, então $U(\mathfrak{g})$ é uma álgebra filtrada. Pelo Teorema de Poincaré-Birkhoff-Witt, $\bar{U}(\mathfrak{g})$ é isomorfo à álgebra de polinômios em $n$ variáveis. Portanto, $U(\mathfrak{g})$ é uma álgebra filtrada especial.

Um famoso teorema de Kostant (veja [Kos63, Theorem 21]) diz que a álgebra envolvente universal de uma álgebra de Lie complexa redutível é um módulo livre sobre seu centro. O resultado mais relevante desta seção é o seguinte teorema principal do artigo [FO05], que é uma versão do Teorema de Kostant para álgebras especiais filtradas.

Teorema 1.5.29. Seja $U$ uma álgebra filtrada especial. Sejam $g_{1}, \ldots, g_{t} \in U$ elementos mutualmente comutativos tais que suas imagens graduadas formam uma sequência regular na álgebra graduada associada à $U$, então $U$ é um $\mathbb{k}\left[g_{1}, \ldots, g_{t}\right]$-módulo livre à esquerda (à direita).

Demonstração. Veja [FO05, Theorem 1.1].

Exemplo 1.5.30. Seja $\mathfrak{g}$ uma álgebra de Lie de dimensão finita sobre $\mathbb{C}$. Vimos que a ação adjunta induz uma estrutura de $\mathfrak{g}$-módulo em $S(\mathfrak{g})$ e $U(\mathfrak{g})$. Sendo assim, podemos considerar a subálgebra $I(\mathfrak{g}) \subset S(\mathfrak{g})$ de $\mathfrak{g}$-invariantes. Pelo Corolário 1.3.18, existe um isomorfismo canônico de $\mathfrak{g}$-módulos entre $S(\mathfrak{g})$ e $U(\mathfrak{g})$, chamado aplicação simétrica. Além disso, quando restrito a $I(\mathfrak{g})$, este isomorfismo determina um isomorfismo entre $I(\mathfrak{g})$ e $Z(\mathfrak{g})$, onde $Z(\mathfrak{g})$ é o centro da álgebra $U(\mathfrak{g})$.

Se $\mathfrak{g}$ é uma álgebra de Lie semissimples, $I(\mathfrak{g})$ admite uma família de geradores algebricamente independentes e homogêneos $f_{1}, \ldots, f_{r}$ (veja [Dix96, Théorème 7.3.8]) tal que $V\left(f_{1}, \ldots, f_{r}\right)$ é irredutível e possui dimensão $n-r$ (veja [Dix96, Théorème 8.1.3]), onde $n$ é a dimensão de $\mathfrak{g}$ e $r$ é o posto de $\mathfrak{g}$. Aplicando o Teorema 1.5.29, temos o famoso Teorema de Kostant (veja [Kos63, Theorem $21])$, que diz que $U(\mathfrak{g})$ é um módulo livre sobre seu centro.

Exemplo 1.5.31. Seja a uma álgebra de Lie simples sobre $\mathbb{k}$, onde $\mathbb{k}$ é um corpo algebricamente fechado de característica zero. Em [RT92], Raïs e Tauvel construíram uma família de $r(m+1)$ elementos algebricamente independentes e homogêneos geradores de $I\left(\mathfrak{a}_{m}\right)$ (veja [RT92, Théorème $4.5]$ ), onde $\mathfrak{a}_{m}=\mathfrak{a} \otimes C[t] /\left\langle t^{m+1}\right\rangle$ e $r$ é o posto de $\mathfrak{a}$. Em [Mus01], Eisenbud e Frenkel foram além disso, eles provaram, utilizando teoria de esquemas, que $I\left(\mathfrak{a}_{m}\right)$ não é só gerado por elementos algebricamente independentes $P_{1}^{(l)}, \ldots, P_{r}^{(l)}, 0 \leq l \leq m$, como provaram que

$$
N_{m}=V\left(P_{1}^{(0)}, \ldots, P_{r}^{(0)}, \ldots, P_{1}^{(m)}, \ldots, P_{r}^{(m)}\right)
$$

é irredutível e é intersecção completa (isto é, possui dimensão $(m+1)(n-r)$ ), onde $n$ é a dimensão de $\mathfrak{a}$ e $r$ é posto de $\mathfrak{a}$ (veja [Mus01, Theorem A.2]). Também foi provado neste artigo que $S\left(\mathfrak{a}_{m}\right)$ é livre como um módulo sobre $I\left(\mathfrak{a}_{m}\right)$, mas considerando o isomorfismo entre $S\left(\mathfrak{a}_{m}\right)$ e $U\left(\mathfrak{a}_{m}\right)$ e aplicando o Teorema 1.5.29, também temos que $U\left(\mathfrak{a}_{m}\right)$ é um módulo livre sobre seu centro. Este último havia sido provado anteriormente por Geoffriau em [Geo94b] supondo $m=1$ e foi conjecturado por ele que era válido para todo $m \geq 1$. 


\section{Capítulo 2}

\section{Álgebras Correntes e Módulos de Harish-Chandra}

Como vimos, representações de peso possuem um papel fundamental na classificação de representações de dimensão finita de álgebras de Lie semissimples. Podemos considerar uma família maior de módulos de álgebras de Lie semissimples: a família dos módulos de peso que possuem espaços de peso de dimensão finita, os quais chamamos de módulos quasi-finitos. Como todo módulo de dimensão finita sobre uma álgebra de Lie semissimples é de peso, temos que cada módulo de dimensão finita sobre uma álgebra de Lie semissimples é um módulo quasi-finito. A classificação dos módulos quasi-finitos para uma álgebra de Lie redutível foi finalizada em [Mat00], onde o artigo [Fer90] e o entendimento dos módulos de pesos uniformemente limitados, módulos quasi-finitos cujas dimensões dos espaços de peso são majoradas por algum número positivo, foram fundamentais.

O estudo de módulos quasi-finitos pode ser feito em outras álgebras que também admitem módulos de peso, como álgebras correntes ( [BLL15,Lau18]), álgebras de Virasoro ( [Mat92,MZ07]), álgebras de Virasoro correntes ( [GLZ11,Sav12]), álgebras de Lie afins (veja a introdução de [DG09]). Tanto quanto para álgebras de Virasoro correntes, quanto para álgebras de Lie afins, o estudo dos módulos de peso uniformemente limitados também teve um papel central na classificação.

Neste capítulo, falaremos sobre as representações das álgebras correntes (generalizadas), que são definidas como sendo o produto tensorial $\mathcal{G}=\mathfrak{g} \otimes A$ de uma álgebra de Lie redutível $\mathfrak{g}$ e uma álgebra afim $A$, estas álgebras incluem as álgebras de Lie redutíveis, álgebras de Takiff, álgebras de loop, álgebras de multiloop, álgebras de $n$-ponto, algumas álgebras de Krichever-Novikov, entre outras. Como álgebras afins possuem unidade, podemos considerar uma subálgebra de Cartan $\mathfrak{h}$ de $\mathfrak{g}$ como sendo uma subálgebra de $\mathcal{G}$ e a representação adjunta torna $\mathcal{G}$ um $\mathfrak{h}$-módulo de peso que possui decomposição em espaços de peso. Portanto, podemos considerar representações de peso sobre $\mathcal{G}$. Um $\mathcal{G}$-módulo de peso simples quasi-finito será chamado de módulo de Harish-Chandra. Este nome é inspirado ao dado a módulos semelhantes no caso de álgebra de Virasoro (veja [Mat92]). Seguindo os artigos [BLL15] e [Lau18], estudaremos os $\mathcal{G}$-módulos de Harish-Chandra.

Na Seção 2.1, começaremos com definições e exemplos. Suporemos que $\mathfrak{g}$ é uma álgebra de Lie simples. Mostraremos uma construção para a extensão central universal $\tilde{\mathcal{G}}$ de $\mathcal{G}$, contruída em [Kas84], além de provar que os elementos centrais da extensão central universal $\tilde{\mathcal{G}}$ agem trivialmente em qualquer $\tilde{\mathcal{G}}$-módulo de Harish-Chandra. Terminaremos a seção estudando o núcleo da representação, veremos que este é sempre da forma $\mathfrak{g} \otimes I$, onde $I$ é um ideal de $A$ tal que $A / I$ possui dimensão finita. Todos estes resultados foram demonstrados ou apresentados em [BLL15], porém daremos alguns exemplos, além de mostrar mais explicitamente algumas demonstrações.

Continuaremos na Seção 2.2 estudando como os elementos da forma $x_{\alpha} \otimes a \in \mathfrak{g}_{\alpha} \otimes A$ agem em um módulo de Harish-Chandra, onde $\alpha$ é um elemento do sistema de raízes $\Phi$ de $\mathfrak{g}$. Em particular, mostraremos que agem ou nilpotentemente ou injetivamente, além mostrar que $x_{\alpha} \otimes a$ age nilpotentemente se, e somente se, $x_{\alpha} \otimes 1$ age nilpotentemente. Isto nos permitirá definir um conjunto parabólico $P \subset \Phi$, que será fundamental no teorema principal da seção: de que todo $\mathcal{G}$-módulo de Harish-Chandra é ou uniformemente limitado ou indução parabólica de um $\mathcal{L}$-módulo 
uniformemente limitado, onde $\mathcal{L}=\mathfrak{l} \otimes A \operatorname{com} \mathfrak{l}=\mathfrak{h} \oplus \sum_{\alpha \in P \cap-P} \mathfrak{g}_{\alpha}$ é uma álgebra de Lie redutível. Todos estes resultados foram demonstrados em [Lau18].

A Seção 2.3 será focada em definir e provar resultados relacionados à módulos de avaliação. Estes são módulos sobre $\mathcal{G}$ construídos a partir de g-módulos e ideais maximais distintos de $A$. Seguiremos [BLL15] para mostrar que uma representação $\phi: \mathcal{G} \rightarrow \operatorname{End}(V)$ é uma representação de um módulo de avaliação se, e somente se, é possível fatorá-la como soma direta finita de representações de $\mathfrak{g}$. Além de demonstrar a existência de uma correspondência biunívoca entre as classes de isomorfismo de $\mathcal{G}$-módulos de avaliação simples e as funções de suporte finito entre o conjunto dos ideais maximais de $A$ para o conjunto de classes de isomorfismo de $\mathfrak{g}$-módulos.

O tema da Seção 2.4 é módulos uniformemente limitados, estes são $\mathcal{G}$-módulos de peso cujos todos os espaços de peso possuem dimensão majorada por algum número positivo. Demonstraremos dois resultados mostrados em [BLL15]. O primeiro de que um módulo de avaliação será uniformemente limitado se, e somente se, até no máximo um dos g-módulos componentes do produto tensorial possui dimensão infinita. E o segundo é que todo $\mathcal{G}$-módulo de Harish-Chandra uniformemente limitado é um módulo de avaliação.

Na Seção 2.5, estudaremos uma relação entre módulos de indução parabólica e módulos de avaliação. A maior parte dos resultados foram provados em [Lau18], porém construímos este isomorfismo mais detalhadamente.

Por fim, na Seção 2.6, vamos aplicar os resultados das seções anteriores e alguns resultados de [ERB17] na teoria de $\mathcal{G}$-módulos de peso máximo. Em particular, mostraremos que dados $r$ módulos de peso máximo sobre $\mathfrak{g}$ e $r$ ideais maximais distintos de $A$, podemos construir um $\mathcal{G}$ módulo de peso máximo de Harish-Chandra.

Durante todo o capítulo, $k$ é um corpo algebricamente fechado de característica zero. Caso não contrariamente especificado, todos os espaços vetoriais, transformações lineares, álgebras e estruturas afins serão consideradas sobre $k$.

\section{1 Álgebras Correntes, Módulos de peso e Extensões Centrais}

Começaremos a seção definindo e exemplificando a álgebra de Lie que iremos estudar.

Definição 2.1.1. Seja $\mathfrak{g}$ uma álgebra de Lie de dimensão finita e $A$ uma k-álgebra comutativa associativa com unidade. A álgebra corrente (generalizada) é a álgebra de Lie $\mathcal{G}=\mathfrak{g} \otimes A$, onde o comutador é dado por

$$
[x \otimes a, y \otimes b]=[x, y] \otimes a b, \quad x, y \in \mathfrak{g}, \quad a, b \in A .
$$

Proposição 2.1.2. O colchete de Lie dado na Definição 2.1.1 realmente define uma estrutura de álgebra de Lie no espaço vetorial $\mathcal{G}=\mathfrak{g} \otimes A$

Demonstração. O colchete de Lie dado está bem definido, pois a aplicação multilinear

$$
\begin{aligned}
\mathfrak{g} \times A \times \mathfrak{g} \otimes A & \rightarrow \mathfrak{g} \otimes A \\
(x, a, y, b) & \mapsto[x, y] \otimes a b
\end{aligned}
$$

está bem definida. Portanto, existe uma transformação linear $(\mathfrak{g} \otimes A) \otimes(\mathfrak{g} \otimes A) \rightarrow \mathfrak{g} \otimes A$ tal que $(x \otimes a) \otimes(y \otimes b) \mapsto[x, y] \otimes a b$. Ou seja, existe uma aplicação bilinear bem definida $\mathcal{G} \times \mathcal{G} \rightarrow \mathcal{G}$ tal que $(x \otimes a, y \otimes b) \mapsto[x, y] \otimes a b$ e essa é o colchete definido acima.

Sejam $x, y, z \in \mathfrak{g}$ e $a, b, c \in A$. Como $\mathfrak{g}$ é uma álgebra de Lie, então $[x, x]=0$ e

$$
[x \otimes a, x \otimes a]=[x, x] \otimes a=0 \otimes a=0 .
$$

Portanto, $\mathcal{G}$ satisfaz a condição de alternatividade. Basta mostrar que $\mathcal{G}$ satisfaz a identidade de Jacobi. Como $A$ é associativa e comutativa,

$$
[x \otimes a,[y \otimes b, z \otimes c]]+[z \otimes c,[x \otimes a, y \otimes b]]+[y \otimes b,[z \otimes c, x \otimes a]]
$$




$$
\begin{aligned}
& =[x,[y, z]] \otimes a(b c)+[z,[x, y]] \otimes c(a b)+[y,[z, x]] \otimes b(c a) \\
& =[x,[y, z]] \otimes a b c+[z,[x, y]] \otimes a b c+[y,[z, x]] \otimes a b c \\
& =([x,[y, z]]+[z,[x, y]]+[y,[z, x]]) \otimes a b c \\
& =0 \otimes a b c=0 .
\end{aligned}
$$

Portanto, a aplicação bilinear definida satisfaz a identidade de Jacobi e a condição de alternatividade, portanto é um colchete de Lie.

Daremos alguns exemplos de álgebras correntes que aparecem na literatura. Cada exemplo mostrado já foi estudado por diferentes motivos.

Exemplo 2.1.3 (Álgebra de Aplicações). Seja $X \subset \mathbb{A}_{\mathbb{k}}^{m}$ um conjunto algébrico afim e $\mathfrak{g}$ uma álgebra de Lie de dimensão finita com base $\left\{e_{i}\right\}_{i=1}^{n}$. Sendo assim, existe uma identificação natural de $\mathfrak{g}$ com o espaço afim $\mathbb{A}_{\mathfrak{k}}^{n}$ dada pela base $\left\{e_{i}\right\}_{i=1}^{n}$. Seja $M(X, \mathfrak{g})$ o k-espaço vetorial dos morfismos de conjuntos algébricos de $X$ em $\mathfrak{g}$, isto é, funções $f: X \rightarrow \mathfrak{g}$ tais que existem polinômios $f_{1}, \ldots, f_{n} \in$ $\mathbb{k}\left[x_{1}, \ldots, x_{m}\right]$ tais que

$$
f\left(a_{1}, \ldots, a_{m}\right)=\sum_{i=1}^{n} f_{i}\left(a_{1}, \ldots, a_{m}\right) e_{i}
$$

para todo $\left(a_{1}, \ldots, a_{m}\right) \in X$. Sendo assim, podemos escrever $f=\sum_{i=1}^{n} f_{i} e_{i}$ para todo $f \in M(X, \mathfrak{g})$. Defina uma estrutura de álgebra de Lie em $M(X, \mathfrak{g})$ pontualmente pela composição

$$
X \longrightarrow \mathfrak{g} \times \mathfrak{g} \stackrel{[,]}{\rightarrow} \mathfrak{g} .
$$

Portanto, se $f=\sum_{i=1}^{n} f_{i} e_{i}$ e $g=\sum_{j=1}^{n} g_{j} e_{j}$, então

$$
[f, g]\left(a_{1}, \ldots, a_{m}\right)=\sum_{i=1}^{n} \sum_{j=1}^{n} f_{i}\left(a_{1}, \ldots, a_{m}\right) g_{j}\left(a_{1}, \ldots, a_{m}\right)\left[e_{i}, e_{j}\right]
$$

para todo $\left(a_{1}, \ldots, a_{m}\right) \in X, \operatorname{logo}\left[f_{i} e_{i}, g_{j} e_{j}\right]=f_{i} g_{j}\left[e_{i}, e_{j}\right]$. Considere a transformação linear

$$
\begin{aligned}
\varphi: M(X, \mathfrak{g}) & \rightarrow \mathfrak{g} \otimes \mathbb{k}[X] \\
\sum_{i=1}^{n} f_{i} e_{i} & \mapsto \sum_{i=1}^{n} e_{i} \otimes f_{i} .
\end{aligned}
$$

Para todo $\sum_{i=1}^{n} f_{i} e_{i}, \sum_{i=1}^{n} g_{j} e_{j} \in M(X, \mathfrak{g})$, temos

$$
\begin{aligned}
\varphi\left(\left[\left(f_{1}, \ldots, f_{n}\right),\left(g_{1}, \ldots, g_{n}\right)\right]\right) & =\sum_{i=1}^{n} \sum_{j=1}^{n} f_{i} g_{j} \varphi\left(\left[e_{i}, e_{j}\right]\right) \\
& =\sum_{i=1}^{n} \sum_{j=1}^{n} f_{i} g_{j}\left(\left[e_{i}, e_{j}\right] \otimes 1\right) \\
& =\sum_{i=1}^{n} \sum_{j=1}^{n}\left[e_{i}, e_{j}\right] \otimes f_{i} g_{j} \\
& =\left[\sum_{i=1}^{n} e_{i} \otimes f_{i}, \sum_{j=1}^{n} e_{j} \otimes g_{j}\right] \\
& =\left[\varphi\left(\sum_{i=1}^{n} f_{i} e_{i}\right), \varphi\left(\sum_{i=1}^{n} g_{j} e_{j}\right)\right] .
\end{aligned}
$$

Logo, $\varphi$ é um homomorfismo de álgebras de Lie. Note que $\sum_{i=1}^{n} f_{i} e_{i} \in \operatorname{ker}(\varphi)$ se, e somente se, 
$f_{i}\left(a_{1}, \ldots, a_{n}\right)=0$ para todo $\left(a_{1}, \ldots, a_{n}\right) \in X$ e $i=1, \ldots, n$ se, e somente se, $f_{1}, \ldots, f_{n} \in I(X)$, onde $I(X)$ é ideal de $\mathbb{k}\left[x_{1}, \ldots, x_{m}\right]$ tal que $\mathbb{k}[X]=\mathbb{k}\left[x_{1}, \ldots, x_{m}\right] / I(X)$. Portanto, $\varphi$ é injetora. Além disso, $e_{j} \otimes f$ é imagem de $f e_{j} \in M(X, \mathfrak{g})$, então $\varphi$ é sobrejetora. Concluímos que $M(X, \mathfrak{g})$ e $\mathfrak{g} \otimes \mathbb{k}[X]$ são isomorfos como álgebras de Lie. A álgebra de Lie $M(X, \mathfrak{g})$ é chamada em [NSS12] de álgebra de aplicações não torcida. É possível definir uma estrutura similar no conjunto dos morfismos de esquemas $X \rightarrow \mathfrak{g}$ ( [Lau14]), onde $X$ é um esquema afim. Se $\mathbb{k}=\mathbb{C}$, é possível definir uma estrutura similar ao conjunto das funções suaves da variedade diferencial $X$ para $\mathfrak{g}$.

Exemplo 2.1.4 (Álgebra corrente). Seja $\mathfrak{g}$ uma álgebra simples e $A=\mathbb{k}\left[x_{1}, \ldots, x_{m}\right]$ a $\mathbb{k}$-álgebra polinomial em $m$ variáveis. Especialmente quando $m=1$, a álgebra de Lie $\mathfrak{g} \otimes A$ é algumas vezes chamada de álgebra corrente polinomial na literatura. A Definição 2.1.1 é uma generalização desta definição, visto que a álgebra polinomial um exemplo clássico de álgebra associativa, comutativa e com unidade. No Exemplo 2.1.3, se tomarmos $X=\mathbb{A}^{n}$, temos que $M\left(\mathbb{A}_{\mathfrak{k}}^{n}, \mathfrak{g}\right) \cong \mathfrak{g} \otimes \mathbb{k}\left[x_{1}, \ldots, x_{n}\right]$.

Exemplo 2.1.5 (Álgebra de Takiff). Seja $\mathfrak{g}$ um álgebra de Lie e $A=\mathbb{k}[t] /\left\langle t^{n+1}\right\rangle$, onde $\left\langle t^{n+1}\right\rangle$ é o ideal de $\mathbb{k}[t]$ gerado por $t^{k}$, a álgebra corrente $\mathfrak{g} \otimes A$ é conhecida como álgebra de Takiff generalizada ou álgebra corrente truncada. Quando $n=1$, esta álgebra é conhecida como álgebra de Takiff.

Exemplo 2.1.6 (Álgebras de multiloop não torcidas). Seja $A=\mathbb{k}\left[t_{1}^{ \pm}, \ldots, t_{n}^{ \pm}\right]$a $\mathbb{k}$-álgebra de polinômios de Laurent em $n$ variáveis, então $\mathfrak{g} \otimes A$ é uma álgebra corrente, também conhecida como álgebra de multiloop não torcida. No caso em que $n=1$, esta álgebra é geralmente chamada de álgebra de loop. Note que o homomorfismo de álgebras $\psi: \mathbb{k}\left[t, t^{-1}\right] \rightarrow \mathbb{k}[X]$ dado por $\psi(t)=y-i x$ e $\psi\left(t^{-1}\right)=y+i x$ é um isomorfismo, onde $i$ é uma raiz quadrada de $-1 \mathrm{em} \mathbb{k} \mathrm{e}$ $X=\mathrm{S}^{1}=\left\{(x, y) \in \mathbb{k}^{2} \mid x^{2}+y^{2}=1\right\}$. Sendo assim, pelo exemplo 2.1.3, então

$$
M\left(\mathrm{~S}^{1}, \mathfrak{g}\right) \cong \mathfrak{g} \otimes \mathbb{k}\left[\mathrm{S}^{1}\right] \cong \mathfrak{g} \otimes \mathbb{k}\left[t, t^{-1}\right] .
$$

Se $n>1$, considere o $n$-toro dado por $T^{n}=\prod_{i=1}^{n} \mathrm{~S}^{1}$, então

$$
M\left(T^{n}, \mathfrak{g}\right) \cong \mathfrak{g} \otimes \mathbb{k}\left[T^{n}\right] \cong \mathfrak{g} \otimes \mathbb{k}\left[\prod_{i=1}^{n} \mathrm{~S}^{1}\right] \cong \mathfrak{g} \otimes\left(\bigotimes_{i=1}^{n} \mathbb{k}\left[\mathrm{S}^{1}\right]\right) \cong \mathfrak{g} \otimes \mathbb{k}\left[t_{1}^{ \pm}, \ldots, t_{n}^{ \pm}\right]
$$

Exemplo 2.1.7 (Álgebras correntes de tipo Krichever-Novikov e Álgebra de $n$-ponto). Suponha $\mathbb{k}=\mathbb{C}$ e seja $\Sigma$ uma superfície de Riemman compacta de genus $g$. Seja $I=\left\{P_{1}, \ldots, P_{n}\right\}$ um conjunto $n$ pontos distintos de $\Sigma$. Seja $A$ a álgebra de funções meromórficas em $\Sigma$ que é holomorfa exceto nos pontos em $I$. A álgebra $\mathcal{G}=\mathfrak{g} \otimes A$ é chamada de álgebra corrente de tipo KricheverNovikov (veja [KN87]). Quando $\Sigma$ é a esfera de Riemman $(g=0)$, a extensão central universal de $\mathcal{G}$ é chamada de álgebra de $n$-ponto (veja [Bre95]). Neste caso, se $n=2$, temos que a álgebra corrente de tipo Krichever-Novikov associada é $\mathcal{G}=\mathfrak{g} \otimes \mathbb{C}\left[t, t^{-1}\right]$ a álgebra de loop.

A partir de agora, suponhamos que $\mathfrak{g}$ é uma álgebra de Lie simples e $\mathfrak{h}$ uma subálgebra de Cartan. Denote por $\Phi$ o conjunto das raízes de $\mathfrak{g}$. Lembre que $\mathfrak{g}$ admite uma decomposição em espaços de pesos quando visto como $\mathfrak{h}$-módulo em relação a representação adjunta e seus pesos são exatamente $\Phi \cup\{0\}$. Para cada $\alpha \in \Phi$, fixe $x_{\alpha} \in \mathfrak{g}_{\alpha}, x_{-\alpha} \in \mathfrak{g}_{-\alpha}$ e $h_{\alpha} \in \mathfrak{h}$ tais que $\left[x_{\alpha}, x_{-\alpha}\right]=h_{\alpha}$ e $\left[h_{\alpha}, x_{\alpha}\right]=2 x_{\alpha}$. Note que $h_{-\alpha}=-h_{\alpha}$. Denotaremos por $\kappa(x, y)$ a forma de Killing de $x, y \in \mathfrak{g}$. Lembre que, dada uma base de raízes simples $\Delta \subset \Phi, \mathfrak{g}$ admite uma decomposição triangular

$$
\mathfrak{g}=\mathfrak{g}_{-} \oplus \mathfrak{h} \oplus \mathfrak{g}_{+},
$$

onde $\mathfrak{g}_{ \pm}=\sum_{\alpha \in \Phi_{ \pm}} \mathfrak{g}_{\alpha}$, onde $\Phi_{+}$e $\Phi_{-}$denotam o conjunto de raízes positivas e negativas, respectivamente.

Além disso, fixamos uma $\mathbb{k}$-álgebra $A$ associativa, comutativa, com unidade e finitamente gerada. Denote por $\operatorname{Specm}(A)$ o conjunto de todos os ideais maximais de $A$. Como $\mathbb{k}$ é algebricamente fechado, pelo Teorema dos Zeros de Hilbert (Teorema 1.1.12), $A / m \cong \mathbb{k}$ para todo $m \in \operatorname{Specm}(A)$. 
Pelo Teorema Chinês do Resto (Teorema 1.1.13),

$$
A /\left(m_{1} \ldots m_{r}\right) \cong A / m_{1} \times \cdots \times A / m_{r} \cong \mathbb{k}^{r},
$$

para todo $m_{1}, \ldots, m_{r} \in \operatorname{Specm}(A)$ tais que $m_{i} \neq m_{j}$ se $i \neq j$. Ambos resultados serão importantes neste trabalho.

Considere a álgebra corrente $\mathcal{G}=\mathfrak{g} \otimes A$. Para todo $h \otimes 1 \in \mathfrak{h} \otimes 1 \subset \mathcal{G}, \alpha \in \Phi$ e $x \otimes a \in \mathfrak{g}_{\alpha} \otimes A$, temos que

$$
[h \otimes 1, x \otimes a]=[h, x] \otimes a=\alpha(h)(x \otimes a) .
$$

Além disso, $[h \otimes 1, x \otimes a]=0$, para todo $h, x \in \mathfrak{h}$ e $a \in A$. Portanto, $\mathcal{G}$ é um $\mathfrak{h} \cong \mathfrak{h} \otimes 1$-módulo que admite decomposição em espaços de peso e seus pesos são exatamente $\Phi \cup\{0\}$. Dada uma base de raízes simples $\Delta \subset \Phi$, temos que $\mathcal{G}$ admite uma espécie de decomposição triangular

$$
\mathcal{G}=\mathcal{G}_{-} \oplus(\mathfrak{h} \otimes A) \oplus \mathcal{G}_{+},
$$

onde $\mathcal{G}_{ \pm}=\mathfrak{g}_{ \pm} \otimes A$. Em alguns casos, é possível dar uma decomposição triangular para $\mathcal{G}$ diferente desta, mas não trataremos deste assunto aqui.

Agora introduziremos o objeto de estudo deste capítulo. Muitas vezes neste capítulo, usaremos o isomorfismo $\mathfrak{h} \otimes 1=\mathfrak{h} \otimes \mathbb{C} \cong \mathfrak{h}$.

Definição 2.1.8. Seja $V$ um $\mathcal{G}$-módulo. Dizemos que $V$ é um $\mathcal{G}$-módulo de peso, se $V$, quando visto como $\mathfrak{h} \otimes 1$-módulo, admite decomposição em espaços de peso, isto é,

$$
V=\bigoplus_{\lambda \in \mathfrak{h}^{*}} V_{\lambda}
$$

onde $V_{\lambda}=\{v \in V \mid(h \otimes 1) v=\lambda(h) v$ para todo $h \in \mathfrak{h}\}$. Se $\lambda \in V$ é tal que $V_{\lambda} \neq 0$, chamaremos $\lambda$ de peso e $V_{\lambda}$ de espaço de peso $\lambda$. Denotaremos por $\Pi(V)$ o conjunto de todos os pesos de $V$. Se $V$ é $\mathcal{G}$-módulo de peso tal que $V_{\lambda}$ possui dimensão finita para todo $\lambda \in \mathfrak{h}^{*}$, dizemos que $V$ é quasi-finito. Se $V$ é um $\mathcal{G}$-módulo quasi-finito tal que existe $N \in Z_{>0}$ tal que $\operatorname{dim} V_{\lambda} \leq N$ para todo $\lambda \in \Pi(V)$, dizemos que $V$ é uniformemente limitado. Se $V$ é um módulo quasi-finito simples, então dizemos que $V$ é um $\mathcal{G}$-módulo de Harish-Chandra.

A definição acima pode ser dada mesmo quando g é semissimples ou redutível. Também podemos utilizar a mesma definição para qualquer extensão central de $\mathcal{G}$. Note que usando a notação da Seção 1.3.2, temos que $(\mathcal{G}, \mathfrak{h} \otimes \mathbb{C})$ é um par de álgebras de Lie válido. Como $A$ é finitamente gerada, temos que $A$ possui dimensão enumerável como espaço vetorial, implicando que $\mathcal{G}$ também possui dimensão enumerável. Em particular, a Definição 2.1.8 e a Definição 1.3.31 são compatíveis.

O principal objetivo deste capítulo é estudar os $\mathcal{G}$-módulos de Harish-Chandra, principalmente os definidos a partir de $\mathfrak{g}$-módulos. Qualquer $\mathcal{G}$-módulo também é um módulo para qualquer extensão central de $\mathcal{G}$, basta supor que a parte central age trivialmente. Mostraremos que a parte central sempre age trivialmente em módulos de Harish-Chandra sobre extensões centrais de $\mathcal{G}$.

A álgebra de Lie $\mathcal{G}$ admite uma extensão central universal, que denotaremos por $\tilde{\mathcal{G}}$. Temos que $\tilde{\mathcal{G}}$ pode ser construída explicitamente. Seguiremos a construção apresentada em [ERB17], mas que foi provada em [Kas84]. Considere o espaço vetorial $A \otimes A$ e $Q$ o subespaço gerado pelos elementos

$$
\{a \otimes b+b \otimes a, a b \otimes c+c a \otimes b+b c \otimes a \mid a, b, c \in A\} .
$$

Denote por $\langle A, A\rangle$ o espaço vetorial quociente $(A \otimes A) / Q$ e por $\langle r, s\rangle$ o elemento $r \otimes s+Q \in\langle A, A\rangle$. A álgebra de Lie $\tilde{\mathcal{G}}$ é isomorfa à álgebra de Lie dada pelo espaço vetorial $\mathfrak{g} \otimes A \oplus\langle A, A\rangle$ e o colchete de Lie

$$
[x \otimes a+\langle r, s\rangle, y \otimes b+\langle t, u\rangle]=[x, y] \otimes a b+\kappa(x, y)\langle a, b\rangle,
$$

para todo $x, y \in \mathfrak{g}$ e $a, b, r, s, t, u \in A$.

Exemplo 2.1.9 (Álgebras de Lie afins). Seja $A=\mathbb{k}\left[t, t^{-1}\right]$, iremos calcular $\langle A, A\rangle$ para determinar 
a extensão central universal da álgebra de loop. Como $\langle\rangle:, A \otimes A \rightarrow\langle A, A\rangle$ é bilinear, basta calcular $\left\langle t^{r}, t^{s}\right\rangle$, onde $r, s \in \mathbb{Z}$. Para todo $r, s \in \mathbb{Z}$,

$$
0=\left\langle t^{r}, t^{s}\right\rangle+\left\langle t^{r+1}, 1\right\rangle+\left\langle t^{s}, t^{r}\right\rangle .
$$

Como $\left\langle t^{r}, t^{s}\right\rangle+\left\langle t^{s}, t^{r}\right\rangle=0$, temos que $\left\langle t^{k}, 1\right\rangle=0$ para todo $k \in \mathbb{Z}$. Além disso,

$$
\begin{aligned}
\left\langle t^{r}, t^{s}\right\rangle & =\left\langle t t^{r-1}, t^{s}\right\rangle \\
& =\left\langle t^{r-1}, t^{s+1}\right\rangle+\left\langle t, t^{r+s-1}\right\rangle \\
& =-\left\langle t^{s+2}, t^{r-2}\right\rangle-\left\langle t^{r+s-1}, t\right\rangle+\left\langle t, t^{r+s-1}\right\rangle \\
& =\left\langle t^{r-2}, t^{s+2}\right\rangle+2\left\langle t, t^{r+s-1}\right\rangle .
\end{aligned}
$$

Sendo assim, é possível mostrar, por indução, que para todo $k \in \mathbb{Z}_{\geq 0}$,

$$
\left\langle t^{r}, t^{s}\right\rangle=\left\langle t^{r-k}, t^{s+k}\right\rangle+k\left\langle t, t^{r+s-1}\right\rangle .
$$

Se $s=-r$, então $\left\langle t^{r}, t^{-r}\right\rangle=r\left\langle t^{1}, t^{-1}\right\rangle$. Suponha $s \neq-r$. Como $\left\langle t^{r}, t^{s}\right\rangle=-\left\langle t^{s}, t^{r}\right\rangle$ e $\left\langle 1, t^{k}\right\rangle=0$ para todo $k \in \mathbb{Z}$, temos que

$$
\begin{aligned}
r\left\langle t, t^{r+1-s}\right\rangle & =\left\langle t^{r}, t^{s}\right\rangle \\
& =-\left\langle t^{s}, t^{r}\right\rangle \\
& =-s\left\langle t, t^{r+s-1}\right\rangle
\end{aligned}
$$

Implicando que $(r+s)\left\langle t, t^{r+s-1}\right\rangle=0$. Como $r+s \neq 0$, segue que $\left\langle t, t^{r+s-1}\right\rangle=0$ e, portanto, $\left\langle t^{r}, t^{s}\right\rangle=0$. Logo, $\langle A, A\rangle$ é unidimensional, gerado por $c=\left\langle t, t^{-1}\right\rangle \mathrm{e}$

$$
\tilde{\mathcal{G}}=\mathfrak{g} \otimes \mathbb{k}\left[t, t^{-1}\right] \oplus \mathbb{k} c,
$$

onde o colchete é dado por

$$
\left[x \otimes t^{r}+\alpha c, y \otimes t^{s}+\beta c\right]=[x, y] \otimes a b+\kappa(x, y) r \delta_{r+s, 0} c,
$$

para todo $x, y \in \mathfrak{g}, r, s \in \mathbb{Z}$ e $\alpha, \beta \in \mathbb{k}$.

Agora iremos para o resultado final dessa seção que, no fundo, diz que o estudo dos módulos de Harish-Chandra sobre $\mathcal{G}$ é suficiente para o estudo dos módulos de Harish-Chandra sobre $\tilde{\mathcal{G}}$. O argumento apresentado aqui é o mesmo ao dado em [BLL15].

Teorema 2.1.10 ( [BLL15, Theorem 2.2]). Seja V um $\tilde{\mathcal{G}}$-módulo de Harish-Chandra. Se uma das seguintes condições é satisfeita:

(i) $V$ possui dimensão finita,

(ii) A possui dimensão finita,

(iii) k é um conjunto de cardinalidade não enumerável,

então $\langle A, A\rangle$ age como zero em $V$.

Demonstração. Fixe $a, b \in A$. Como $A$ é finitamente gerada, temos que $A$ é um espaço vetorial de dimensão enumerável sobre $\mathbb{k}$. Como $\mathfrak{g}$ possui dimensão finita, temos que $\mathcal{G}=\mathfrak{g} \otimes A$ possui dimensão enumerável, portanto $\tilde{\mathcal{G}}$ também. Como $V$ é simples e $\tilde{\mathcal{G}}$ possui dimensão enumerável, temos que $V$ é gerado, como espaço vetorial, pelo conjunto

$$
\{x v \mid x \in U(\tilde{\mathcal{G}})\}
$$


e este possui subconjunto gerador de $V$ enumerável. O elemento central de $\tilde{\mathcal{G}}\langle a, b\rangle$ age em $V$ por um endomorfismo $T \in \operatorname{Hom}_{\tilde{\mathcal{G}}}(V, V)$, então, pelo Lema de Schur (Lema 1.3.5), existe escalar $\lambda_{a, b} \in \mathbb{k}$ tal que $T=\lambda_{a, b} I d$, onde $a, b \in A$ e $I d$ é aplicação identidade.

Seja $\mu \in \Pi(V)$, então $V_{\mu}$ possui dimensão finita. Como a forma de Killing é não degenerada quando restrita à subálgebra de Cartan $\mathfrak{h}$, temos que existem $h_{1}, h_{2} \in \mathfrak{h}$ tais que $\kappa\left(h_{1}, h_{2}\right) \neq 0$. O traço do endomorfismo

$$
\left(h_{1} \otimes a\right)\left(h_{2} \otimes b\right)-\left(h_{2} \otimes b\right)\left(h_{1} \otimes a\right)
$$

é zero quando restrita a $V_{\mu}$, mas $\left[h_{1} \otimes a, h_{2} \otimes b\right]=\kappa\left(h_{1}, h_{2}\right)\langle a, b\rangle$ age pelo escalar $\kappa\left(h_{1}, h_{2}\right) \lambda_{a, b}$. Sendo assim, o traço do endomorfismo acima restrito à $V_{\mu}$ é $\operatorname{dim}\left(V_{\mu}\right) \kappa\left(h_{1}, h_{2}\right) \lambda_{a, b}$. Como $V_{\mu}$ é não nulo e $\kappa\left(h_{1}, h_{2}\right) \neq 0$, temos que $\langle a, b\rangle$ age como zero em $V_{\mu}$, portanto age como zero em todo espaço de peso de $V$. Logo, $\langle a, b\rangle$ age como zero em $V$.

\subsubsection{O Núcleo da Representação}

Seja $V$ um módulo de Harish-Chandra para $\mathcal{G}$, cuja representação está associada à representação $\rho: \mathcal{G} \rightarrow$ End $V$. Terminaremos a seção estudando o núcleo de $\rho$. Porém, antes disto, determinaremos quais são os ideais de $\mathcal{G}$. Lembre que $\mathfrak{g}$ é uma álgebra de Lie simples de dimensão finita sobre um corpo $\mathbb{k}$ algebricamente fechado de característica zero.

Proposição 2.1.11. Seja $P$ um ideal de $\mathcal{G}$, então existe $I$ ideal de $A$ tal que $P=\mathfrak{g} \otimes I$.

Demonstração. Note que $[\mathfrak{g} \otimes 1, x \otimes a]=\mathfrak{g} \otimes a$ para todo $x \in \mathfrak{g}$ não nulo, pois $\mathfrak{g}$ é simples. Sendo assim, se $x \otimes a \in P$ e $y \otimes b \in P$, então $z \otimes\left(c_{1} a+c_{2} b\right) \in P$ para todo $z \in \mathfrak{g}, c_{1}, c_{2} \in \mathbb{k}$. Além disso, se $x \otimes a \in P$, então $[x, y] \otimes b a \in P$ para todo $y \in \mathfrak{g}$ e $b \in A$. Logo, se $x \otimes a \in P$, então $x \otimes a b \in P$ para todo $b \in A$. Portanto, se mostrarmos que $P$ é gerado, como espaço vetorial, por um conjunto da forma $\{x \otimes a \mid a \in S, x \in B\}$ para uma base $B$ de $\mathfrak{g}$, então $P=\mathfrak{g} \otimes\langle S\rangle$, onde $\langle S\rangle$ é o ideal de $A$ gerado por $S$.

Fixe $\mathfrak{h}$ uma subálgebra de Cartan de $\mathfrak{g}$ e $\alpha_{1} \ldots, \alpha_{r}$ um sistema de raízes simples. Sejam $0 \neq$ $x_{\alpha} \in \mathfrak{g}_{\alpha}$ e $\left\{h_{1}, \ldots, h_{r}\right\}$ uma base de $\mathfrak{h}$ tais que $\left[x_{\alpha_{i}}, x_{-\alpha_{i}}\right]=h_{i}$, para cada $i=1, \ldots, r$. Seja $v \in P$ não nulo e escreva

$$
v=\sum_{\alpha \in \Phi} x_{\alpha} \otimes a_{\alpha}+\sum_{i=1}^{r} h_{i} \otimes a_{i} .
$$

Logo, para todo $k>0$ e todo $h \in \mathfrak{h}$,

$$
0 \neq(\operatorname{ad} h)^{k}(v)=\sum_{\alpha \in \Phi} \alpha(h)^{k} x_{\alpha} \otimes a_{\alpha} \in P .
$$

Como existe $h \in \mathfrak{h}$ tal que $\alpha(h) \neq 0$ para todo $\alpha \in \Phi$ e $\alpha(h) \neq \beta(h)$ se $\alpha, \beta \in \Phi$ são distintos, então, pelo determinante de Vandermonde, $x_{\alpha} \otimes a_{\alpha} \in P$ e $\sum_{i=1}^{r} h_{i} \otimes a_{i} \in P$. Como

$$
\left[\sum_{i=1}^{r} h_{i} \otimes a_{i}, x_{\alpha_{j}} \otimes 1\right]=\sum_{i=1}^{r} \alpha_{j}\left(h_{i}\right) x_{\alpha_{i}} \otimes a_{i}
$$

e $h_{i} \otimes a_{i}=\left[x_{\alpha_{i}}, x_{-\alpha_{i}}\right] \otimes a_{i}$, temos que $h_{i} \otimes a_{i} \in P$. Pelo argumento dado no primeiro parágrafo, $\mathfrak{g} \otimes a_{\alpha} \subset P$ e $\mathfrak{g} \otimes a_{i} \subset P$, para todo $\alpha \in \Phi$ e $i=1, \ldots, r$. Denote por $S_{v}=\left\{a_{\alpha} \mid \alpha \in \Phi\right\} \cup\left\{a_{i} \mid i=\right.$ $1, \ldots, r\}$. Faça esse processo para todo $v \in P$.

Logo, se $S=\bigcup_{v \in P} S_{v} \subset A$, então $P$ é gerado, como espaço vetorial, pelo conjunto $\mathfrak{g} \otimes S$. Pelo argumento dado no primeiro parágrafo, $P$ é igual o conjunto $\mathfrak{g} \otimes I$, onde $I$ é o ideal de $A$ gerado por $S$.

Observação 2.1.12. Na Proposição 2.1.11, provamos que, se $\mathfrak{g}$ é simples, os ideais de $\mathfrak{g} \otimes A$ são da forma $\mathfrak{g} \otimes I$, onde $I$ é um ideal de $A$. Se $\mathfrak{g}$ é semissimples ou redutível, teremos um caso diferente. Seja $\mathfrak{s}$ uma álgebra de Lie semissimples e $L_{1}, \ldots, L_{p} \subset \mathfrak{s}$ ideais que são subálgebras de Lie simples 
de $\mathfrak{s}$ tais que $\mathfrak{s}=L_{1} \oplus \cdots \oplus L_{p}$, como álgebra de Lie. Seja $\mathfrak{z}$ uma álgebra de Lie abeliana de dimensão finita e considere a álgebra redutível dada pela soma direta $\mathfrak{r}=\mathfrak{s} \oplus \mathfrak{z}$. Como $\mathfrak{z} \otimes A$ é uma álgebra de Lie abeliana, todo subconjunto $J$ de $\mathfrak{z} \otimes A$ é um ideal de $\mathfrak{z} \otimes A$. Então utilizando a Proposição 2.1.11 e que $\mathfrak{s}$ é uma soma direta de álgebras de Lie simples, os ideais de $\mathfrak{s} \otimes A$ e $\mathfrak{r} \otimes A$ serão da forma $\bigoplus_{i=1}^{p} L_{i} \otimes I_{i}$ e $\bigoplus_{i=1}^{p} L_{i} \otimes I_{i} \oplus J$, respectivamente, onde $I_{1}, \ldots, I_{p}$ são ideais de A e $J$ é um subconjunto de $\mathfrak{z} \otimes A$.

Pela Proposição 2.1.11, existe um ideal $I=I(V)$ de $A$ tal que ker $\rho=\mathfrak{g} \otimes I(V)$. Para cada $\alpha \in \Phi \cup\{0\}, v \in V$ e $\lambda \in \mathfrak{h}^{*}$, defina os espaços vetoriais

$$
\begin{gathered}
J_{\alpha}(v)=\left\{a \in A \mid(x \otimes a) v=0 \text { tal que } x \in \mathfrak{g}_{\alpha}\right\}, \\
J(v)=\bigcap_{\beta \in \Phi \cup\{0\}} J_{\beta}(v), \\
J(\lambda)=\bigcap_{w \in V_{\lambda}} J(w), \\
J(\lambda, \Phi)=\bigcap_{\mu \in \lambda+(\Phi \cup\{0\})} J(\mu) .
\end{gathered}
$$

Lema 2.1.13. O espaço vetorial $J(\lambda, \Phi)$ possui codimensão finita em A para todo $\lambda \in \mathfrak{h}^{*}$. Isto é, o espaço vetorial $A / J(\lambda, \Phi)$ possui dimensão finita.

Demonstração. Fixe $\lambda \in \mathfrak{h}^{*}$. Como os espaços de peso de $V$ possuem dimensão finita, $V_{\lambda}$ possui dimensão finita. Seja $\left\{v_{i}\right\}_{i=1}^{k}$ uma base $V_{\lambda}$, então $J(\lambda)=\bigcap_{i=1}^{k} J\left(v_{i}\right)$. Como

$$
J\left(v_{i}\right)=\bigcap_{\beta \in \Phi \cup\{0\}}\left\{a \in A:(x \otimes a) v_{i}=0 \text { tal que } x \in \mathfrak{g}_{\alpha}\right\}
$$

e $\Phi$ é um conjunto finito, temos que $J(\lambda, \Phi)$ pode ser escrito como uma intesecção finita de subespaços da forma $J_{\alpha}(v)$.

Para cada $x \in \mathfrak{g}_{\alpha}$ e $v \in V_{\lambda}$, considere a transformação linear

$$
\begin{aligned}
\eta_{x, v}: A & \rightarrow V_{\lambda+\alpha} \\
a & \mapsto(x \otimes a) v .
\end{aligned}
$$

Pelo Teorema do Homomorfismo, existe uma transformação linear injetora de $A / \operatorname{ker}\left(\eta_{x, v}\right)$ em $V_{\lambda+\alpha}$, que possui dimensão finita. Logo, o espaço vetorial $A / \operatorname{ker}\left(\eta_{x, v}\right)$ possui dimensão finita. Seja $B_{\alpha}=$ $\left\{x_{\alpha}\right\}$, se $\alpha \in \Phi$, e $B_{0}=\left\{h_{\alpha}: \alpha \in \Delta\right\}$, onde $\Delta$ é um sistema de raízes simples de $\Phi$. Para todo $v \in V_{\lambda}, J_{\alpha}(v)=\bigcap_{x \in B_{\alpha}}$ ker $\eta_{x, v}$. Sendo assim,

$$
J(\lambda, \Phi)=\bigcap_{i=1}^{k} \bigcap_{\alpha \in \Phi \cup\{0\}} \bigcap_{x \in B_{\alpha}} \operatorname{ker} \eta_{x, v_{i}} .
$$

é uma intersecção finita de subespaços. Em particular, a transformação linear

$$
\begin{aligned}
A / J(\lambda, \Phi) & \rightarrow \bigoplus_{\substack{x \in B_{\alpha} \\
i=1, \ldots, n}} A / \operatorname{ker} \eta_{x, v_{i}} \\
a+J(\lambda, \Phi) & \mapsto\left(a+\operatorname{ker} \eta_{x, v_{i}}\right)_{x \in B_{\alpha}, i=1, \ldots, n}
\end{aligned}
$$

é injetora. Como cada $A / \eta_{x, v}$ na soma direta acima possui dimensão finita, segue que $J(\lambda, \Phi)$ tem codimensão finita.

Lema 2.1.14. $J(\lambda, \Phi) A \subset J(\lambda)$, para cada peso $\lambda$ de $V$. 
Demonstraçãa. Fixe $\lambda \in \mathfrak{h}^{*}$ tal que $V_{\lambda} \neq 0$. Sejam $r \in J(\lambda, \Phi), a \in A$ e $v \in V_{\lambda}$. Tome $x \in \mathfrak{g}_{\alpha}$ e $y \in \mathfrak{g}_{\beta}$, onde $\alpha, \beta \in \Phi \cup\{0\}$. Note que $(y \otimes s) v \in V_{\lambda+\beta}$. Pela definição de $J(\lambda, \Phi),(x \otimes r) v=$ $(x \otimes r)(y \otimes s) v=0$. Sendo assim,

$$
\begin{aligned}
([x, y] \otimes r s) & =[x \otimes r, y \otimes s] \\
& =(x \otimes r)(y \otimes s) v-(y \otimes s)(x \otimes r) v=0 .
\end{aligned}
$$

Como $\mathfrak{g}$ é simples, $[\mathfrak{g}, \mathfrak{g}]=\mathfrak{g}, \log 0(\mathfrak{g} \otimes r s) v=([\mathfrak{g}, \mathfrak{g}] \otimes r s) v=0$. Em particular, $r s \in J(\lambda)$.

Proposição 2.1.15 ( [BLL15, Proposition 4.3]). Seja $\lambda \in \mathfrak{h}^{*}$ um peso de um $\mathcal{G}$-módulo de HarishChandra $V$, então $I(V)=J(\lambda, \Phi)$. Em particular, $A / I(V)$ possui dimensão finita.

Demonstração. Note que $I(V) \subset J(\lambda, \Phi)$, pois para todo $x \in \mathfrak{g}$ e $a \in I(V),(x \otimes a) v=0$.

Sejam $v \in V_{\lambda}, v \neq 0$. Como $V$ é simples e $v$ é não nulo, segue que $U(\mathcal{G}) v=V$. Portanto, para todo $w \in V$, existem $k \geq 0, x_{1}, \ldots, x_{k} \in \mathfrak{g}$ e $a_{1}, \ldots, a_{k} \in A$ tais que $w=\left(x_{1} \otimes a_{1}\right) \ldots\left(x_{k} \otimes a_{k}\right) v$.

Vamos provar, por indução em $k$, que para todo $x \in \mathfrak{g}, r \in J(\lambda, \Phi)$ e $a \in A$, temos que

$$
(x \otimes r a)\left(x_{1} \otimes a_{1}\right) \ldots\left(x_{k} \otimes a_{k}\right) v=0 .
$$

Pelo Lemma 2.1.14, segue que $(x \otimes r a) v=0$, pois $r a \in J(\lambda)$, para todo $r \in J(\lambda, \Phi)$ e $a \in A$. Suponha $k \geq 1$, então

$$
\begin{aligned}
(x \otimes r a)\left(x_{1} \otimes a_{1}\right) \ldots\left(x_{r} \otimes a_{r}\right) v= & \left(x_{1} \otimes a_{1}\right)(x \otimes r a)\left(x_{2} \otimes a_{2}\right) \ldots\left(x_{r} \otimes a_{r}\right) v \\
& +\left(\left[x, x_{1}\right] \otimes r a a_{1}\right)\left(x_{2} \otimes a_{2}\right) \ldots\left(x_{r} \otimes a_{r}\right) v .
\end{aligned}
$$

Pela hipótese de indução, ambos somandos do lado direito da igualdade acima são zero, já que $r a a_{1} \in J(\lambda, \Phi) A=J(\lambda)$. Sendo assim, $(x \otimes r a) w=0$, para todo $x \in \mathfrak{g}, r \in J(\lambda, \Phi)$ e $a \in A$. Em particular, se $a=1,(x \otimes r) w=0$, para todo $x \in \mathfrak{g}$ e $r \in J(\lambda, \Phi)$. Sendo assim, $J(\lambda, \Phi)=I(V)$. Pelo Lema 2.1.13, $J(\lambda, \Phi)$ tem codimensão finita, portanto $A / I(V)$ é um álgebra de dimensão finita.

Observação 2.1.16. Sejam $\mathfrak{g}_{1}, \ldots, \mathfrak{g}_{k}$ álgebras de Lie simples de dimensão finita e $\mathfrak{z}$ uma álgebra de Lie abeliana de dimensão finita. Seja $\mathfrak{l}$ a álgebra de Lie redutível dada pela soma direta de álgebras de Lie $\mathfrak{l}=\mathfrak{g}_{1} \oplus \cdots \oplus \mathfrak{g}_{k} \oplus \mathfrak{z}$. Se $V$ é um $\mathfrak{l} \otimes A$-módulo de Harish-Chandra, então, pela demonstração da Proposição 1.3.34, existe um homomorfismo de $\mathfrak{l} \otimes A$-módulos

$$
\Psi: V_{1} \otimes \cdots \otimes V_{k} \otimes W \rightarrow V
$$

sobrejetor, onde $V_{i}$ é um $\mathfrak{g}_{i} \otimes A$-módulo de Harish-Chandra, $i=1, \ldots, n$, e $W$ é um $\mathfrak{z} \otimes A$-módulo de dimensão 1. Como $\mathfrak{g}_{i}$ é uma álgebra de Lie simples de dimensão finita, pela Proposição 2.1.15, podemos ver $V_{i}$ como uma representação da álgebra de Lie de dimensão finita $\mathfrak{g}_{i} \otimes A / I\left(V_{i}\right)$. Portanto, pela Proposição 1.3.22, $V_{1} \otimes \cdots \otimes V_{k} \otimes W$ é um módulo simples sobre

$$
\left(\mathfrak{g}_{1} \otimes A / I\left(V_{1}\right)\right) \oplus \cdots \oplus\left(\mathfrak{g}_{k} \otimes A / I\left(V_{k}\right)\right) \oplus(\mathfrak{z} \otimes A),
$$

o que implica que $V_{1} \otimes \cdots \otimes V_{k} \otimes W$ é um $\mathfrak{l} \otimes A$-módulo simples também. Logo, $\Psi$ é um isomorfismo de $\mathfrak{l} \otimes A$-módulos.

Proposição 2.1.17. Seja $\mathfrak{l}$ uma álgebra de Lie redutivel de dimensão finita e A uma álgebra comutativa associativa com unidade e finitamente gerada. Suponha que $\mathfrak{l}=\mathfrak{z} \oplus \bigoplus_{i=1}^{k} \mathfrak{g}_{i}$, onde $\mathfrak{g}_{1}, \ldots, \mathfrak{g}_{k}$ são ideais simples de $\mathfrak{l}$ e $\mathfrak{z}$ é o centro de $\mathfrak{l}$. Seja $V$ um módulo de Harish-Chandra sobre a álgebra corrente $\mathfrak{l} \otimes A$. Então, para cada $i=1, \ldots, k$, existe um $\mathfrak{g}_{i}$-módulo de Harish-Chandra $V_{i}$ e um $\mathfrak{z}$-módulo simples unidimensional $W$ tal que $V \cong W \otimes \bigotimes_{i=1}^{k} V_{i}$, onde

$$
\left(z+x_{1}+\ldots x_{k}\right)\left(w \otimes v_{1} \otimes \cdots \otimes v_{k}\right)=(z w) \otimes v_{1}, \ldots, v_{k}+\sum_{i=1}^{k} w \otimes v_{1} \otimes \cdots \otimes x_{i} v_{i} \otimes \cdots \otimes v_{k},
$$


$z \in \mathfrak{z}, x_{i} \in \mathfrak{g}_{i}, w \in W$ e $v_{i} \in V_{i}$, para $i=1, \ldots, k$.

Demonstração. Basta utilizar o argumento dado na Observação 2.1.16 e no Corolário 1.3.35.

Portanto, todos os resultados adquiridos neste capítulo, podem ser generalizados supondo $\mathfrak{g}$ redutível.

\subsection{Módulos de Harish-Chandra e Indução Parabólica}

Nesta seção, mostraremos uma classificação de $\mathcal{G}$-módulos de Harish-Chandra. Começaremos mostrando que $x_{\alpha} \otimes a$ ou age nilpotentemente ou age injetivamente em um $\mathcal{G}$-módulo de HarishChandra, para todo $\alpha \in \Phi$ e $a \in A$. Além disso, mostraremos que $x_{\alpha} \otimes a$ age nilpotentemente se, e somente se, $x_{\alpha} \otimes 1$ age nilpotentemente. Isto nos permitirá dividir o sistema de raízes em dois subconjuntos, os quais utilizaremos para construir um subconjunto parabólico $P \subset \Phi$. Este conjunto parabólico será utilizado para mostrar que, na prática, todo $\mathcal{G}$-módulo de Harish-Chandra é indução parabólica de um $\mathcal{L}$-módulo uniformemente limitado, onde $\mathcal{L}$ será uma subálgebra de $\mathcal{G}$ determinada por $P$.

Definição 2.2.1. Seja $L$ uma álgebra de Lie e $\rho: L \rightarrow \mathfrak{g l}(V)$ um $L$-módulo. Dizemos que $x \in L$ age nilpotentemente em $v \in V$ se existe $k \geq 0$ tal que $\rho(x)^{k} v=0$. Dizemos que $x \in L$ age injetivamente em $V$ se $\rho(x): V \rightarrow V$ é uma transformação linear injetora.

Lema 2.2.2. Seja $L$ um álgebra de Lie. Para todo $x, y \in L$,

$$
x^{k} y=y x^{k}+\sum_{r=1}^{k}\left(\begin{array}{c}
k \\
k-r
\end{array}\right)\left(\operatorname{ad}_{x}\right)^{r}(y) x^{k-r}
$$

quando visto como elementos de $U(L)$.

Demonstração. Provaremos por indução em $k$. Se $k=1$, segue que

$$
x y=y x+[x, y]=y x+\left(\operatorname{ad}_{x}\right)(y) .
$$

Suponha que $k>1$. Pela hipótese de indução, para todo $x, y \in L$ temos que

$$
x^{k-1} y=y x^{k-1}+\sum_{r=1}^{k-1}\left(\begin{array}{c}
k-1 \\
k-1-r
\end{array}\right)\left(\operatorname{ad}_{x}\right)^{r}(y) x^{k-1-r}
$$

Como a hipótese de indução é verdade para todo $y \in \mathfrak{g}$, segue que também é para $[x, y] \in L$ e portanto

$$
\begin{aligned}
x^{k-1}[x, y] & =[x, y] x^{k-1}+\sum_{r=1}^{k-1}\left(\begin{array}{c}
k-1 \\
k-1-r
\end{array}\right)\left(\left(\operatorname{ad}_{x}\right)\right)^{r} x^{k-1-r} \\
& =\sum_{r=1}^{k}\left(\begin{array}{l}
k-1 \\
k-r
\end{array}\right)\left(\operatorname{ad}_{x}\right)^{r} x^{k-r} .
\end{aligned}
$$

Logo,

$$
\begin{aligned}
x^{k} y & =x^{k-1} y x+x^{k-1}[x, y] \\
& =y x^{k-1}+\sum_{r=1}^{k-1}\left(\left(\begin{array}{c}
k-1 \\
k-1-r
\end{array}\right)+\left(\begin{array}{l}
k-1 \\
k-r
\end{array}\right)\right)\left(\operatorname{ad}_{x}\right)^{r}(y) x^{k-r}+\left(\operatorname{ad}_{x}\right)^{k}(y) \\
& =y x^{k}+\sum_{r=1}^{k}\left(\begin{array}{c}
k \\
k-r
\end{array}\right)\left(\operatorname{ad}_{x}\right)^{r}(y) x^{k-r} .
\end{aligned}
$$


Lema 2.2.3. Seja $V$ um $\mathcal{G}$-módulo de Harish-Chandra. Sejam $\alpha \in \Phi$ e a $\in$ A não nulo, então ou $x_{\alpha} \otimes$ a age nilpotentemente em todo elemento de $V$ ou age injetivamente em $V$.

Demonstração. Suponha que $x_{\alpha} \otimes a$ não aja injetivamente em $V$, então o subespaço vetorial de $W \subset V$ dado por

$$
W=\left\{v \in V \mid\left(x_{\alpha} \otimes a\right)^{k} v=0 \text { para algum } k \in \mathbb{Z}_{>0}\right\}
$$

é não nulo. Sejam $y \otimes b \in \mathcal{G}$ e $w \in W$. Como $\operatorname{ad}_{x_{\alpha}}$ age nilpotentemente em $y$, segue que existe $k_{1} \in \mathbb{Z}_{>0}$ tal que $\left(\operatorname{ad}_{x_{\alpha}}\right)^{k_{1}}(y)=0$. Além disso, como $w \in W$, existe $k_{2} \in \mathbb{Z}_{>0}$ tal que $\left(x_{\alpha} \otimes a\right)^{k_{2}} w=0$. Pelo Lema 2.2.2, se $k=k_{1}+k_{2}+1$, temos

$$
\begin{aligned}
\left(x_{\alpha} \otimes a\right)^{k}(y \otimes b) w & =(y \otimes b)\left(x_{\alpha} \otimes a\right)^{k} w+\sum_{r=1}^{k}\left(\begin{array}{c}
k \\
k-r
\end{array}\right) \operatorname{ad}_{x_{\alpha} \otimes a}^{r}(y \otimes b)\left(x_{\alpha} \otimes a\right)^{k-r} w \\
& =\sum_{r=1}^{k}\left(\begin{array}{c}
k \\
k-r
\end{array}\right)\left(\operatorname{ad}_{x_{\alpha} \otimes a}\right)^{r}(y \otimes b)\left(x_{\alpha} \otimes a\right)^{k-r} w \\
& =0
\end{aligned}
$$

pois ou $r>k_{1}$ ou $k-r>k_{2}$, para todo $r=1, \ldots, k$.

Logo, $W$ é um submódulo não nulo de $V$. Como $V$ é simples, segue que $W=V$ e $x_{\alpha} \otimes a$ age nilpotentemente em todos os elementos de $V$.

A seguinte Proposição foi provada em [Lau18] adaptando argumentos dados em [Fer90].

Proposição 2.2.4 ( [Lau18, Proposition 2.2]). Sejam V um $\mathcal{G}$-módulo de Harish-Chandra e $\alpha \in \Phi$. Denote por $\Pi(V)$ o conjunto dos pesos de $V$, então são equivalentes

(i) Para todo $\lambda \in \Pi(V), V_{\lambda+n \alpha} \neq 0$ apenas para uma quantidade finita de $n>0$.

(ii) Existe um $\lambda \in \Pi(V)$ tal que $V_{\lambda+n \alpha} \neq 0$ apenas para uma quantidade finita de $n>0$.

(iii) Para todo $a \in A$ não nulo, o elemento $x_{\alpha} \otimes$ a age localmente nilpotentemente em $V$.

(iv) $O$ elemento $x_{\alpha} \otimes 1$ age localmente nilpotentemente em $V$.

Demonstração. Obviamente, (i) implica (ii). Além disso, tomando $a=1$, claramente (iii) implica (iv). Suponha (ii) e seja $\lambda \in \Pi(V)$ tal que os espaços de peso $V_{\lambda+n \alpha}$ é não zero apenas para uma quantidade finita de $n>0$. Isto é, existe $N>0$ tal que $V_{\lambda+n \alpha}=0$ para todo $n>N$. Portanto, $x_{\alpha} \otimes a$ age nilpotentemente no espaço não nulo $V_{\lambda}$, para cada $a \in A$. Pelo Lema 2.2.3, segue que $x_{\alpha} \otimes a$ age nilpotentemente em todo $V$.

Basta mostrar que (iv) implica (i). Suponha que $x_{\alpha} \otimes 1$ aja localmente nilpotentemente em $V$. Seja $\lambda \in \Pi(V)$ e suponha que $V_{\lambda+n \alpha}$ é não zero para uma quantidade infinita de $n>0$. Como $x_{\alpha} \otimes 1$ age nilpotentemente, existe uma sequência de vetores $v_{i} \in V_{\lambda+n_{i} \alpha}$ tal que $\left(x_{\alpha} \otimes 1\right) v_{i}=0$, onde $n_{1}<n_{2}<n_{3}<\ldots$

Denote por $\mathfrak{s}_{\alpha}$ a subálgebra de $\mathcal{G}$ gerada por $x_{-\alpha} \otimes 1, h_{\alpha} \otimes 1, x_{\alpha} \otimes 1$, então $\mathfrak{s}_{\alpha}$ é isomorfa à $\mathfrak{s l}_{2}$. Note que $U\left(\mathfrak{s}_{\alpha}\right) v_{i}$ é um $\mathfrak{s}_{\alpha}$-módulo de peso máximo $\lambda+n_{i} \alpha$. Seja $N$ um inteiro positivo tal que $(\lambda+N \alpha)\left(h_{\alpha}\right) \notin \mathbb{Z}_{\leq 0}$. Portanto, para todo $n_{i}>N$ existe $w_{i} \in U\left(s_{\alpha}\right) v_{i}$ vetor não nulo de peso $\lambda+N \alpha$. Para cada $\beta \in \mathfrak{h}^{*}$, denote por $\chi_{\beta}$ o caractere central de $\beta$ e seja $I \subset Z_{>0}$ tal que $n_{i}, n_{j}>N$ e $\chi_{\lambda+n_{i} \alpha} \neq \chi_{\alpha+n_{j} \alpha}$, para todo $i, j \in I, i \neq j$. Como foi comentado, cada um dos $U\left(\mathfrak{s}_{\alpha}\right) v_{i}$ é um $s_{\alpha}$-módulo de peso máximo $\lambda+n_{i} \alpha$ e, como são todos distintos entre si, temos que $I$ é um conjunto infinito. Vamos provar que $\left\{w_{i} \mid i \in I\right\}$ é um conjunto linearmente independente. Seja

$$
C=\frac{\left(h_{\alpha} \otimes 1\right)^{2}}{2}+\left(x_{\alpha} \otimes 1\right)\left(x_{-\alpha} \otimes 1\right)+\left(x_{-\alpha} \otimes 1\right)\left(x_{\alpha} \otimes 1\right) \in U\left(\mathfrak{s}_{\alpha}\right)
$$


o elemento de Casimir de $U\left(\mathfrak{s}_{\alpha}\right)$, então $C$ é um elemento do centro da álgebra $U\left(\mathfrak{s}_{\alpha}\right)$ e assim $C w=\chi_{\lambda+n_{i} \alpha}(C) w$, para todo $i \in I$ e $w \in U\left(\mathfrak{s}_{\alpha}\right) v_{i}$. Além disso, como a subálgebra gerada por $C$ é o centro de $U\left(\mathfrak{s}_{\alpha}\right)$, segue que $\chi_{\lambda+n_{i} \alpha}(C) \neq \chi_{\lambda+n_{j} \alpha}(C)$ para todo $i, j \in I, i \neq j$. Sejam $i_{1}<\cdots<i_{l} \in I$ e $c_{i_{1}}, \ldots, c_{i_{l}} \in \mathbb{k}$ tais que $\sum_{r=1}^{l} c_{i_{r}} w_{i_{r}}=0$. Para todo $k \geq 0$, temos que

$$
0=C^{k}\left(\sum_{r=1}^{l} c_{i_{r}} w_{i_{r}}\right)=\sum_{r=1}^{l} c_{i_{r}} \chi_{\lambda+n_{i_{r}} \alpha}(C)^{k} w_{i_{r}},
$$

portanto $c_{i_{r}}=0$, para $r=1, \ldots, l$. Logo, $\left\{w_{i} \mid i \in I\right\} \subset V_{\lambda+N \alpha}$ é um conjunto infinito e linearmente independente, contradizendo que a hipótese que os espaços de peso em $V$ possuem dimensão finita. Portanto, o espaço de peso $V_{\lambda+n \alpha}$ é não zero apenas para uma quantidade finita de $n>0$.

Corolário 2.2.5. Sejam $V$ um $\mathcal{G}$-módulo de Harish-Chandra e $\alpha \in \Phi$. São equivalentes

(i) Para todo $\lambda \in \Pi(V), V_{\lambda+n \alpha} \neq 0$ para todo $n>0$.

(ii) Existe um $\lambda \in \Pi(V)$ tal que $V_{\lambda+n \alpha} \neq 0$ para todo $n>0$.

(iii) Para todo a $\in A$ não nulo, o elemento $x_{\alpha} \otimes$ a age injetivamente em $V$.

(iv) $O$ elemento $x_{\alpha} \otimes 1$ age injetivamente em $V$.

Demonstração. Basta aplicar o Lema 2.2.3 na Proposição 2.2.4.

Definição 2.2.6. Seja $V$ um módulo de peso. Uma raiz $\alpha \in \Phi$ é chamada de localmente finita em $V$ se $x_{\alpha} \otimes 1$ age nilpotentemente em $V$. Denotaremos por $\Phi^{f}=\Phi^{f}(V)$ o conjunto das raízes localmente finitas e $\Phi^{i}=\Phi^{i}(V)=\Phi \backslash \Phi^{f}$ seu complemento. Uma raiz $\alpha \in \Phi^{i}$ é chamada de raiz injetivas em $V$.

Nos próximos lemas e proposições, utilizaremos a notação e resultados dados na Seção 1.2.3.

Lema 2.2.7. Seja $V$ um $\mathcal{G}$-módulo de Harish-Chandra, então

(i) $\Phi^{i} \subset \Phi$ é fechado.

(ii) $\Phi^{f} \subset \Phi$ é fechado.

(iii) Sejam $\alpha \in \Phi^{f} \cap-\Phi^{f} \quad$ e $\beta \in \Phi^{i} \cap-\Phi^{i}$, então $\alpha+\beta \notin \Phi$.

(iv) $P=\Phi^{f} \cup-\Phi^{i} e-P=-\left(\Phi^{f} \cup-\Phi^{i}\right)=\left(-\Phi^{f}\right) \cup \Phi^{i}$ são fechados.

(v) $P \backslash-P$ é fechado.

Demonstração. (i) Sejam $\alpha, \beta \in \Phi^{i}$ tais que $\alpha+\beta \in \Phi$. Pelo Lema 2.2.3 e Proposição 2.2.4, temos que $x_{\alpha} \otimes 1$ e $x_{\beta} \otimes 1$ agem injetivamente em $V$. Como composição de endomorfismos injetores é injetor, segue que $\left(x_{\alpha} \otimes 1\right)\left(x_{\beta} \otimes 1\right)$ age injetivamente em $V$. Sendo assim, $V_{\lambda+n(\alpha+\beta)}$ é não nulo para todo $\lambda \in \Pi(V)$ e $n \geq 0$. Pelo Corolário 2.2.5, $\alpha+\beta$ é injetiva em $V$.

(ii) Usaremos [Fer90, Corollary 2.7] que afirma que se a é uma álgebra de Lie de dimensão finita e $W$ é um a-módulo finitamente gerado, então

$$
\mathfrak{a}[W]=\{x \in \mathfrak{a} \mid x \text { age nilpotentemente em } \mathrm{W}\}
$$

é uma subálgebra de $\mathfrak{a}$.

Seja $\mathfrak{a}$ a subálgebra de Lie de $\mathfrak{g} \otimes 1$ gerada por $x_{\alpha} \otimes 1$ e $x_{\beta} \otimes 1$. Seja $v \in V$ um vetor não nulo de peso $\lambda$ e seja $U(\mathfrak{a}) v=W \subset V$ o a-submódulo de $V$ gerado por $v$. A álgebra de Lie $\mathfrak{a}$ possui dimensão finita e $W$ é um a-módulo finitamente gerado. Por [Fer90][Corollary 2.7], os elementos de $\mathfrak{a}$ que agem nilpotentemente em $W$ formam uma subálgebra de $\mathfrak{a}$. Como $x_{\alpha+\beta} \otimes 1$ é um múltiplo não nulo de $\left[x_{\alpha} \otimes 1, x_{\beta} \otimes 1\right]$, segue que $x_{\alpha+\beta} \otimes 1$ age nilpotentemente em $W$. Pelo Lema 2.2.3, $x_{\alpha+\beta}$ age nilpotentemente em todo $V$ e, portanto, $\alpha+\beta \in \Phi^{f}$. 
(iii) Suponha que $\alpha+\beta \in \Phi$. Se $\alpha+\beta \in \Phi^{f}$, então, pelo item (ii) deste lema, $\beta=-\alpha+(\alpha+\beta) \in \Phi^{i}$, o que é uma contradição. Por outro lado, se $\alpha+\beta \in \Phi^{i}$, então, pelo item (i) deste lema, $\alpha=-\beta+(\alpha+\beta) \in \Phi^{f}$, o que também é uma contradição. Segue que $\alpha+\beta \notin \Phi$.

(iv) Sejam $\alpha, \beta \in \Phi^{f} \cup-\Phi^{i}$. Se $\alpha, \beta \in \Phi^{f}$ ou $\alpha, \beta \in-\Phi^{i}$, então, se $\alpha+\beta \in \Phi$, segue que $\alpha+\beta \in \Phi^{f} \cup-\Phi^{i}$, pelo item (i) ou (ii) deste lema. Suponha que $\alpha \in \Phi^{f} \backslash-\Phi^{i}$ e $\beta \in\left(-\Phi^{i}\right) \backslash \Phi^{f}$. Mostraremos que $\alpha+\beta \notin \Phi$. Note que $\Phi=\Phi^{f} \cup \Phi^{i}=\left(-\Phi^{f}\right) \cup\left(-\Phi^{i}\right)$ e essas uniões são disjuntas. Assim, $\Phi^{f}=\left(\left(-\Phi^{f}\right) \cap \Phi^{f}\right) \cup\left(\left(-\Phi^{i}\right) \cap \Phi^{f}\right)$ e $-\Phi^{i}=\left(\Phi^{f} \cap-\Phi^{i}\right) \cup\left(\Phi^{i} \cap-\Phi^{i}\right)$. Como $\alpha \in \Phi^{f} \backslash-\Phi^{i}$ e $\beta \in\left(-\Phi^{i}\right) \backslash \Phi^{f}$, temos que $\alpha \in\left(-\Phi^{f}\right) \cap \Phi^{f}$ e $\beta \in \Phi^{i} \cap-\Phi^{i}$. Pelo item (iii) deste lema, $\alpha+\beta \notin \Phi$. Sendo assim, $P$ é fechado. Podemos dar o mesmo argumento e provar que $-P$ é fechado também.

(v) Sejam $\alpha, \beta \in P \backslash-P$ tais que $\alpha+\beta \in \Phi$. Pelo item (iv), $\alpha+\beta \in P$. Basta mostrar que $\alpha+\beta \notin-P$. Suponha que $\alpha+\beta \in-P$. Como $\alpha+\beta \in P \cap-P$, temos que $-\alpha-\beta \in P$. Logo, $-\alpha=(-\alpha-\beta)+\alpha \in P$ contradiz o fato de que $\alpha \in P \backslash-P$. Portanto $\alpha+\beta \notin-P$.

Lema 2.2.8. Seja $V$ um $\mathcal{G}$-módulo de Harish-Chandra. Seja $P=\Phi^{f} \cup-\Phi^{i}$, então $\Phi^{i} \cap-\Phi^{i}$, $\Phi^{f} \cap-\Phi^{f}$ e $P \cap-P$ são sistemas de raízes para o espaço vetorial gerado por eles. Além disso, $P \subset \Phi$ é um conjunto parabólico em $\Phi$.

Demonstração. Note que $\Phi^{i} \cap-\Phi^{i}, \Phi^{f} \cap-\Phi^{f}$ e $P \cap-P$ são todos simétricos. Além disso, pelo Lema 2.2.7, são todos fechados. Sendo assim, pela Proposição 1.2.35, eles são todos sistemas raízes para o espaço vetorial gerado por eles.

Proposição 2.2.9. Sejam $V$ um $\mathcal{G}$-módulo de Harish-Chandra e $P=\Phi^{f} \cup-\Phi^{i}$, então os subespaços

$$
\begin{gathered}
\mathfrak{l}=\mathfrak{h} \oplus \sum_{\alpha \in P \cap-P} \mathfrak{g}_{\alpha}, \\
\mathfrak{n}_{ \pm}=\sum_{\alpha \in P \backslash-P} \mathfrak{g}_{ \pm \alpha} \\
\mathfrak{p}=\mathfrak{l} \oplus \mathfrak{n}_{+}
\end{gathered}
$$

são subálgebras de Lie de $\mathfrak{g}$. Temos ainda que $\mathfrak{l}$ é uma álgebra de Lie redutível com subálgebra de Cartan $\mathfrak{h}$ e sistema de raízes $P \cap-P$. Além disso, $\mathfrak{l}=\mathfrak{s} \oplus \mathfrak{z}$, onde $\mathfrak{s}=\operatorname{span}\left\{h_{\alpha} \mid \alpha \in\right.$ $P \cap-P\} \oplus \bigoplus_{\alpha \in P \cap-P} \mathfrak{g}_{\alpha}$ é uma álgebra de Lie semissimples, $\mathfrak{z}=\operatorname{span}\left\{h_{\alpha} \mid \alpha \in P \backslash-P\right\}$ é o centro de $\mathfrak{l}$ e $\mathfrak{h}=\mathfrak{z} \oplus \operatorname{span}\left\{h_{\alpha} \mid \alpha \in P \cap-P\right\}$. Temos ainda que $\mathfrak{g}=\mathfrak{n}_{-} \oplus \mathfrak{p}$ e $\mathfrak{p}$ é uma subálgebra de Lie parabólica, isto é, $\mathfrak{p}$ é uma subálgebra de $\mathfrak{g}$ que contém uma subálgebra de Borel.

Demonstração. Pelo Lema 2.2.8, $P \subset \Phi$ é um conjunto parabólico no sistema de raízes $\Phi$. Pela Proposição 1.2.33, $\mathfrak{p}$ é uma subálgebra de $\mathfrak{g}$. Ainda pelo Lema 2.2.8, $\mathfrak{s}=\operatorname{span}\left\{h_{\alpha} \mid \alpha \in P \cap\right.$ $-P\} \oplus \bigoplus_{\alpha \in P \cap-P} \mathfrak{g}_{\alpha}$ é uma álgebra de Lie semissimples com sistema de raízes $P \cap-P$. Como $\mathfrak{z}=\operatorname{span}\left\{h_{\alpha} \mid \alpha \in P \backslash-P\right\}$ comuta com todos os elementos de $\mathfrak{s}$, temos que $\mathfrak{l}=\mathfrak{s} \oplus \mathfrak{z}$ é uma subálgebra de Lie de $\mathfrak{g}$ e é redutível. Pelo Lema 2.2.7, $P \backslash-P$ é fechado, portanto $-P \backslash P$ também fechado e $\mathfrak{n}_{ \pm}$são subálgebras de $\mathfrak{g}$.

Para um módulo de Harish-Chandra $V$, denotaremos $\mathfrak{l}=\mathfrak{l}(V), \mathfrak{n}_{ \pm}=\mathfrak{n}_{ \pm}(V)$ e $\mathfrak{p}=\mathfrak{p}(V)$ como na Proposição 2.2.9. Além disso, denote por $\mathcal{L}=\mathcal{L}(V)=\mathfrak{l} \otimes A, \mathcal{N}_{ \pm}=\mathcal{N}_{ \pm}(V)=\mathfrak{n}_{ \pm} \otimes A$ e $\mathcal{P}=\mathcal{P}(V)=\mathfrak{p} \otimes A$.

Lembre que denotamos por $\mathcal{Q}$ o reticulado de raízes $\operatorname{span}_{\mathbb{Z}} \Phi$. Denote por $\mathcal{Q}_{0}=\mathcal{Q}_{0}(V)$ o reticulado de raízes gerado por $P \cap-P$. Se $W \subset V$ é $\mathcal{L}$-módulo de Harish-Chandra, então se $\lambda \in \mathfrak{h}^{*}$ é um peso de $W$ todos os pesos de $W$ estão contidos em $\lambda+\mathcal{Q}_{0}$. Podemos ver $W$ como um $\mathcal{P}$-módulo definindo $\mathcal{N}_{+} W=\{0\}$. 
Proposição 2.2.10. Seja $W$ um $\mathcal{L}$-módulo quasi-finito cujos pesos diferem apenas por elementos de $Q_{0}$. Considere o módulo de Verma generalizado

$$
M_{\mathcal{P}}(W)=U(\mathcal{G}) \otimes_{U(\mathcal{P})} W
$$

então

(i) Como espaços vetoriais,

$$
M_{\mathcal{P}}(W) \cong\left(U\left(\mathcal{N}_{-}\right) \mathcal{N}_{-} \otimes W\right) \oplus W .
$$

Além disso, os pesos de $U\left(\mathcal{N}_{-}\right) \mathcal{N}_{-} \otimes W$ e $W$ formam conjuntos disjuntos.

(ii) $M_{\mathcal{P}}(W)$ possui um único submódulo $N_{\mathcal{P}}(W)$ que é maximal entre os submódulos que possuem interseç̧ão trivial com $W$.

(iii) $O$ quociente $L_{\mathcal{P}}(W)=M_{\mathcal{P}}(W) / N_{\mathcal{P}}(W)$ é um $\mathcal{G}$-módulo simples se, e somente se, $W$ é um $\mathcal{L}$-módulo simples.

(iv) $O$ espaço de $\mathcal{N}_{+}$-invariantes em $L_{\mathcal{P}}(W)$

$$
L_{\mathcal{P}}(W)^{\mathcal{N}_{+}}=\left\{v \in L_{\mathcal{P}}(W) \mid x v=0, \text { para todo } x \in \mathcal{N}_{+}\right\}
$$

é igual a $W$.

Demonstração. Vamos supor $P \neq \Phi$, pois se $P=\Phi$, então $\mathcal{P}=\mathcal{G}$ e $M_{\mathcal{P}}(W) \cong W$.

(i) Pelo Teorema PBW, como espaços vetoriais,

$$
\begin{aligned}
M_{\mathcal{P}}(W) & =U(\mathcal{G}) \otimes_{U(\mathcal{P})} W \\
& \cong U\left(\mathcal{N}_{-}\right) U(\mathcal{P}) \otimes_{U(\mathcal{P})} W \\
& \cong\left(U\left(\mathcal{N}_{-}\right) \mathcal{N}_{-} \otimes W\right) \oplus W
\end{aligned}
$$

Como $\mathcal{N}_{-}$é um h-módulo de peso com a ação adjunta, temos que $U\left(\mathcal{N}_{-}\right)$é um h-módulo de peso com a ação adjunta também. Explicitamente, se $x_{i} \in\left(\mathcal{N}_{-}\right) \alpha_{i}, \alpha_{i} \in(-P) \backslash P$, $i=1, \ldots, k$, então

$$
\left[h, x_{1} \ldots x_{k}\right]=\left(\alpha_{1}+\cdots+\alpha_{k}\right)(h) x_{1} \ldots x_{k} .
$$

Note que se $x \in U(\mathcal{N})_{\alpha}$ e $w \in W_{\lambda}$, então para todo $h \in \mathfrak{h}$

$$
h x w=x h w+[h, x] w=(\lambda+\alpha)(h) x w .
$$

Isto é, os pesos de $M_{\mathcal{P}}(W)$ são da forma $\lambda+\alpha$, onde $\lambda$ é um peso de $W$ e $\alpha$ é um peso de $U\left(\mathcal{N}_{-}\right)$. Além disso, como $U(\mathcal{N})_{0}=\mathbb{k}$, os pesos de $U\left(\mathcal{N}_{-}\right) \mathcal{N}_{-} \otimes W$ são da forma $\lambda+\alpha$, onde $\alpha$ é um peso de $U\left(\mathcal{N}_{-}\right)$não nulo. Como os pesos de $W$ diferem apenas por elementos de $\mathcal{Q}_{0}$ e os pesos de $U\left(\mathcal{N}_{-}\right) \mathcal{N}_{-}$estão contidos em $\mathcal{Q} \backslash \mathcal{Q}_{0}$, segue que os pesos de $U\left(\mathcal{N}_{-}\right) \mathcal{N}_{-} \otimes W$ e $W$ são distintos.

(ii) Como qualquer submódulo de um módulo de peso é um módulo de peso, temos que qualquer $\mathcal{G}$-submódulo $N \subset M_{\mathcal{P}}(W)$ tal que $N \cap W=0$ está contido em $U\left(\mathcal{N}_{-}\right) \mathcal{N}_{-} \otimes W$. Portanto, se $N_{\mathcal{P}}(W)$ é a soma de todos os $\mathcal{G}$-submódulos de $M_{\mathcal{P}}(W)$ que intersectam $W$ trivialmente, então $N_{\mathcal{P}}(W)$ está contido em $U\left(\mathcal{N}_{-}\right) \mathcal{N}_{-} \otimes W$ e é maximal entre os $\mathcal{G}$-submódulos que possuem interseç̧ão trivial com $W$.

(iii) Seja $U \subset W$ um submódulo não de $W$. Visto como subespaço de $M_{\mathcal{P}}(W)$, o $\mathcal{G}$-submódulo gerado por $U$ em $M_{\mathcal{P}}(W)$ é igual à

$$
U(\mathcal{G}) \otimes_{U(\mathcal{P})} U
$$


Note que a imagem de $W$ na projeção canônica de $M_{\mathcal{P}}(W)$ em $L_{\mathcal{P}}(W)=M_{\mathcal{P}}(W) / N_{\mathcal{P}}(W)$ é isomorfa a $W$, por isso identificaremos $W$ com sua imagem em $L_{\mathcal{P}}(W)$.

Suponha que $W$ não é simples e seja $U \subset W$ um $\mathcal{L}$-submódulo próprio de $W$ não nulo. Pelo item (i), temos que os pesos do submódulo $N_{\mathcal{P}}(W) \subset M_{\mathcal{P}}(W)$ são distintos dos pesos de $W$. Portanto $L_{\mathcal{P}}(W)_{\lambda}=W_{\lambda}$ para cada peso $\lambda$ de $W$. Como $U$ é um submódulo próprio, existe $\lambda$ tal que $U_{\lambda}$ está contido propriamente em $W_{\lambda}$. Sendo assim, se

$$
L=\frac{U(\mathcal{G}) \otimes_{U(\mathcal{P})} U+N_{\mathcal{P}}(W)}{N_{\mathcal{P}}(W)},
$$

então $L_{\lambda}=U_{\lambda}$ e, portanto, $L$ é um $\mathcal{G}$-submódulo não nulo de $L_{\mathcal{P}}(W)$.

Por outro lado, suponha $W$ simples e seja $N \subset L_{\mathcal{P}}(W)$ um $\mathcal{G}$-submódulo. Pela construção de $N_{\mathcal{P}}(w)$, todo submódulo de $L_{\mathcal{P}}(W)$ intersecta com $W$. Assim, se $N \cap W=0$, então $N=0$. Suponha que $N \cap W \neq 0$, então

$$
N \supset U(\mathcal{G})(N \cap W)=U\left(\mathcal{N}_{-}\right) U(\mathcal{P})(N \cap W)=U\left(\mathcal{N}_{-}\right) W=L_{\mathcal{P}}(W) .
$$

Logo, $N=L_{\mathcal{P}}(W)$ e, como vale para todo submódulo não nulo de $L_{\mathcal{P}}(W)$, temos que $L_{\mathcal{P}}(W)$ é simples.

(iv) Seja $v \in L_{\mathcal{P}}(W)$ um vetor de peso tal que $v \in L_{\mathcal{P}}(W)^{\mathcal{N}_{+}}$. Identificando $W$ como sua imagem em $L_{\mathcal{P}}(W), v \in W$ ou $v \in U\left(\mathcal{N}_{-}\right) N_{-} W$ pelo item (i). Por definição $\mathcal{N}_{+} W=0$, temos que $W \subset L_{\mathcal{P}}(W)^{\mathcal{N}_{+}}$. Suponha que $v \in U\left(\mathcal{N}_{-}\right) N_{-} W$. Sendo assim, pelo Teorema de PoincaréBirkhoff-Witt,

$$
\begin{aligned}
U(\mathcal{G}) v & =U\left(\mathcal{N}_{-}\right) U(\mathcal{L}) U\left(\mathcal{N}_{+}\right) v \\
& =U\left(\mathcal{N}_{-}\right) U(\mathcal{L}) v .
\end{aligned}
$$

Se $\alpha \in P \cap-P$ e $\beta \in(-P) \backslash P$ tal que $\alpha+\beta \in \Phi$, então $\alpha+\beta \in(-P) \backslash P$. Logo, $\left[\mathcal{L}, \mathcal{N}_{-}\right] \subset \mathcal{N}_{-}$. Portanto,

$$
\begin{aligned}
U\left(\mathcal{N}_{-}\right) U(\mathcal{L}) v & \subset U\left(\mathcal{N}_{-}\right) U(\mathcal{L}) U\left(\mathcal{N}_{-}\right) N_{-} W \\
& \subset U\left(\mathcal{N}_{-}\right) N_{-} W .
\end{aligned}
$$

Como os pesos de $U\left(\mathcal{N}_{-}\right) N_{-} W$ e $W$ são distintos, temos que $U(\mathcal{G}) v \cap W=0$. Pela construção de $N_{\mathcal{P}}(W)$, temos que $U(\mathcal{G}) v=0$. Concluímos que $L_{\mathcal{P}}(W)^{\mathcal{N}_{+}}=W$.

Lema 2.2.11. Seja $V$ um $\mathcal{G}$-módulo de Harish-Chandra. Se $P=\Phi$, então $\Phi=\Phi^{f}$ ou $\Phi=\Phi^{i}$.

Demonstração. Lembre que $P=\Phi^{f} \cup-\Phi^{i}$. Seja $\alpha \in \Phi^{f}$, então $-\alpha \notin-\Phi^{i}$. Como $P=\Phi$, então $\Phi \backslash-\Phi^{i}=P \backslash-\Phi^{i} \subset \Phi^{f}$. Sendo assim, $\alpha \in \Phi^{f} \cap-\Phi^{f}$. Da mesma forma, se $\alpha \in-\Phi^{i}$, temos que $\alpha \in \Phi^{i} \cap-\Phi^{i}$. Logo,

$$
P=\Phi=\left(\Phi^{f} \cap-\Phi^{f}\right) \cup\left(\Phi^{i} \cap-\Phi^{i}\right)
$$

e esta união é disjunta, pois $\Phi^{f}$ e $\Phi^{i}$ são disjuntos. Pela Proposição 1.2.35, $\Phi^{f} \cap-\Phi^{f}$ e $\Phi^{i} \cap-\Phi^{i}$ são subsistemas de raízes de $\Phi$, pois são fechados, pela Proposição 2.2.7, e simétricos. Pelo Lema 2.2.7, para todo $\alpha \in \Phi^{f} \cap-\Phi^{f}$ e $\beta \in \Phi^{i} \cap-\Phi^{i}, \alpha+\beta \notin \Phi$ e $\alpha-\beta \notin \Phi$, portanto $\alpha$ e $\beta$ são raízes ortogonais. Como g é simples, temos que $\Phi$ é um sistema de raízes irredutível (isto é, não pode ser particionado em uma união de dois subconjuntos tais que as raízes do primeiro são ortogonais às raízes do segundo) e, assim, $\Phi=\Phi^{f} \cap-\Phi^{f}$ ou $\Phi=\Phi^{i} \cap-\Phi^{i}$. Concluímos que $\Phi=\Phi^{f}$ ou $\Phi=\Phi^{i}$.

Teorema 2.2.12 ( [Lau18, Theorem 2.9]). Seja V um $\mathcal{G}$-móulo de Harish-Chandra, então um dos casos mutualmente exclusivos a seguir ocorre 
(i) $\Phi=\Phi^{f}$ e $V$ possui dimensão finita;

(ii) $\Phi=\Phi^{i}$ e $V$ é um módulo uniformemente limitado de dimensão infinita, com $\operatorname{dim}\left(V_{\lambda}\right)=$ $\operatorname{dim}\left(V_{\mu}\right)$, para todo $\lambda, \mu \in \Pi(V)$;

(iii) $\Phi$ não é igual à $\Phi^{f}$ ou $\Phi^{i}$. Neste caso, a subálgebra parabólica correspondente $\mathcal{P}$ está contida propriamente em $\mathcal{G}$,

$$
V^{\mathcal{N}_{+}}=\left\{v \in V \mid x v=0 \text { para todo } x \in \mathcal{N}_{+}\right\}
$$

é um $\mathcal{L}$-módulo uniformemente limitado simples e $V \cong L_{\mathcal{P}}\left(V^{\mathcal{N}_{+}}\right)$.

Demonstração. (i) Suponha que $\Phi=\Phi^{f}$. Mostraremos que $V$ possui dimensão finita. Para isso, enumere as raízes $\Phi=\left\{\alpha_{1}, \ldots, \alpha_{t}\right\}$ e defina

$$
W_{i}(\lambda)=U\left(\mathfrak{g}_{\alpha_{i}} \otimes A\right) \otimes \cdots \otimes U\left(\mathfrak{g}_{\alpha_{1}} \otimes A\right) U(\mathfrak{h} \otimes A) V_{\lambda},
$$

onde $i=1, \ldots, t$ e $\lambda \in \Pi(V)$. Como $W_{i}(\lambda)$ é um $\mathfrak{h} \otimes 1$-módulo de peso, podemos definir $S_{i}(\lambda)$ como sendo o conjunto de seus pesos. Como $V$ é irredutível, pelo Teorema de PoincaréBirkhoff-Witt, $W_{t}(\lambda)=V$ e $S_{t}(\lambda)=\Pi(V)$, para todo $\lambda \in \Pi(V)$. Fixe $\lambda \in \Pi(V)$. Pela Proposição 2.2.4, o conjunto $S_{1}(\lambda)$ é finito. Suponha, por indução, que $S_{i}(\lambda)$ seja finito. Novamente, pela Proposição 2.2.4, para cada $\gamma \in S_{i}(\lambda)$, o conjunto $\Pi(V) \cap\left\{\gamma+n \alpha_{i} \mid n \in \mathbb{Z}_{\geq 0}\right\}$ é finito. Logo,

$$
S_{i+1}(\lambda)=\bigcup_{\gamma \in S_{i}(\lambda)} \Pi(V) \cap\left\{\gamma+n \alpha_{i} \mid n \in \mathbb{Z}_{\geq 0}\right\}
$$

é uma união finita de conjuntos finitos, portanto $S_{i+1}(\lambda)$ é finito. Em particular, $S_{t}(\lambda)=\Pi(V)$ é um conjunto finito. Como os espaços de peso de $V$ possuem dimensão finita e $V$ possui uma quantidade finita de pesos, segue que $V$ possui dimensão finita.

(ii) Suponha que $\Phi=\Phi^{i}$. Sejam $\alpha \in \Phi$ e $\lambda \in \Pi(V)$. Como $-\alpha \in \Phi^{i}, V_{\alpha+n \alpha} \neq 0$ para todo $n \in \mathbb{Z}$ pelo Corolário 2.2.5. Logo, $V$ possui dimensão infinita. O elemento $x_{\alpha} \otimes 1$ age injetivamente em $V$, pois $\alpha \in \Phi^{i}$, portanto a aplicação

$$
\begin{aligned}
V_{\lambda} & \rightarrow V_{\lambda+\alpha} \\
v & \mapsto\left(x_{\alpha} \otimes 1\right) v
\end{aligned}
$$

é injetora, implicando que $\operatorname{dim}\left(V_{\lambda}\right) \leq \operatorname{dim}\left(V_{\lambda+\alpha}\right)$. Por outro lado, $-\alpha \in \Phi$ também é uma raiz injetiva em $V$, então a aplicação

$$
\begin{aligned}
V_{\lambda+\alpha} & \rightarrow V_{\lambda} \\
v & \mapsto\left(x_{-\alpha} \otimes 1\right) v
\end{aligned}
$$

é injetora e $\operatorname{dim}\left(V_{\lambda}\right) \geq \operatorname{dim}\left(V_{\lambda+\alpha}\right)$. Assim, $\operatorname{dim}\left(V_{\lambda}\right)=\operatorname{dim}\left(V_{\lambda+\alpha}\right)$. Fazendo o mesmo argumento, podemos provar que $\operatorname{dim} V_{\lambda}=\operatorname{dim} V_{\mu}$ para todo $\mu \in \lambda+\mathcal{Q}$. Como $V$ é simples, $V_{\lambda}$ gera $V$, portanto $\Pi(V)=\lambda+\mathcal{Q}$, concluindo a prova.

(iii) Suponha que $\Phi$ não é igual à $\Phi^{i}$ ou $\Phi^{f}$, então $V$ é não nulo. Pelo Lema $2.2 .11, P \neq \Phi$ e, assim, $\mathcal{P}$ é uma subálgebra própria de $\mathcal{G}$. Como $\left[\mathcal{L}, \mathcal{N}_{+}\right] \subset \mathcal{N}_{+}$, temos que $V^{\mathcal{N}_{+}}$é um $\mathcal{L}$-submódulo de $V$. Primeiramente, mostraremos que $V^{\mathcal{N}_{+}}$é não nulo. Seja $v \in V$ um vetor não nulo de peso $\lambda$ e considere o $\mathcal{N}_{+}$-módulo $U\left(\mathcal{N}_{+}\right) v$. Lembre que

$$
\mathcal{N}_{+}=\bigoplus_{\alpha \in P \backslash-P} \mathfrak{g}_{\alpha} \otimes A
$$

Se $P \backslash-P=\left\{\beta_{1}, \ldots, \beta_{k}\right\}$, então, pelo Teorema Poincaré-Birkhoff-Witt,

$$
U\left(\mathcal{N}_{+}\right) v=U\left(\mathfrak{g}_{\beta_{k}} \otimes A\right) \ldots U\left(\mathfrak{g}_{\beta_{1}} \otimes A\right) v .
$$


Como $P \backslash-P \subset \Phi^{f}$, utilizando um argumento parecido ao dado no primeiro caso, é possível mostrar que $U\left(\mathcal{N}_{+}\right) v$ possui dimensão finita. Pela Proposição 1.2.33, existe $\Delta \subset \Phi$ base tal que $P \backslash-P$ é um conjunto de raízes positivas. Como $U\left(\mathcal{N}_{+}\right) v$ possui dimensão finita, existe $\mu \in \Pi(V)$ tal que $\left(U\left(\mathcal{N}_{+}\right) v\right)_{\mu} \neq 0$, mas $\left(U\left(\mathcal{N}_{+}\right) v\right)_{\alpha}=0$ para todo $\alpha \in \lambda+\mathcal{Q}_{+}$tal que $\alpha \neq \mu$, onde $\mathcal{Q}_{+}=\left\{\lambda+\sum_{\beta \in \Delta} a_{\beta} \beta \mid a_{\beta} \in \mathbb{Z}_{\geq 0}\right\}$. Sendo assim, $0 \neq\left(U\left(\mathcal{N}_{+}\right) v\right)_{\mu} \subset V^{\mathcal{N}_{+}}$e $V^{\mathcal{N}_{+}} \neq 0$.

Provaremos agora que $V \cong L_{\mathcal{P}}\left(V^{\mathcal{N}_{+}}\right)$. Considere a transformação linear

$$
\begin{aligned}
\varphi: M_{\mathcal{P}}\left(V^{\mathcal{N}_{+}}\right) & \rightarrow V \\
u \otimes v & \mapsto u v,
\end{aligned}
$$

então $\varphi$ é um homomorfismo de álgebras de Lie, pois $\varphi(x(u \otimes v))=\varphi(x u \otimes v)=x \varphi(u \otimes v)$. Além disso, $\varphi$ é sobrejetor, pois sua imagem contém $V^{\mathcal{N}_{+}}$e $V$ é simples. Sendo assim, $V$ é isomorfo à $M_{\mathcal{P}}\left(V^{\mathcal{N}_{+}}\right)$quocientado por $\operatorname{ker}(\varphi)$ como $\mathcal{G}$-módulos, implicando que $\operatorname{ker} \varphi$ é maximal. Pela Proposição 2.2.10,

$$
M_{\mathcal{P}}\left(V^{\mathcal{N}_{+}}\right) \cong\left(U\left(\mathcal{N}_{-}\right) \mathcal{N}_{-} \otimes V^{\mathcal{N}_{+}}\right) \oplus\left(1 \otimes V^{\mathcal{N}_{+}}\right)
$$

como espaços vetoriais. Como $\varphi(1 \otimes v)=v$ para todo $v \in V^{\mathcal{N}_{+}}$, temos que $V^{\mathcal{N}_{+}} \cap \operatorname{ker}(\varphi)=0$ e, portanto, $\operatorname{ker}(\varphi) \subset N_{\mathcal{P}}\left(V^{\mathcal{N}_{+}}\right) . \operatorname{Logo}, \operatorname{ker}(\varphi)=N_{\mathcal{P}}\left(V^{\mathcal{N}_{+}}\right)$e $V \cong L_{\mathcal{P}}\left(V^{\mathcal{N}_{+}}\right)$, porque $N_{\mathcal{P}}\left(V^{\mathcal{N}_{+}}\right)$é maximal entre os ideais que possuem intersecção trivial com $V^{\mathcal{N}_{+}}$. Além disso, pela Proposição 2.2.10, $V^{\mathcal{L}_{+}}$é um $\mathcal{L}$-módulo simples.

Basta mostrar que $V^{\mathcal{N}_{+}}$é um $\mathcal{L}$-módulo uniformemente limitado. O sistema de raízes de $\mathcal{L}$ é

$$
\begin{aligned}
P \cap-P & =\left(\Phi^{f} \cup-\Phi^{i}\right) \cap\left(\Phi^{i} \cup-\Phi^{f}\right) \\
& =\left(\Phi^{f} \cap-\Phi^{f}\right) \cup\left(\Phi^{i} \cap-\Phi^{i}\right) .
\end{aligned}
$$

Como vimos na demonstração do Lema 2.2.11, $\Phi^{f} \cap-\Phi^{f}$ e $\Phi^{i} \cap-\Phi^{i}$ são subsistemas de raízes de $P \cap-P$ e suas raízes são ortogonais entre si. Logo,

$$
\mathfrak{l}=\mathfrak{z} \oplus \mathfrak{g}^{f} \oplus \mathfrak{g}^{i},
$$

onde $\mathfrak{z}=\operatorname{span}\left\{h_{\alpha} \mid \alpha \in P \backslash-P\right\}$ é o centro de $\mathfrak{l}, \mathfrak{g}^{f}=\sum_{\alpha \in \Phi^{f} \cap-\Phi^{f}} \operatorname{span}\left\{h_{\alpha}, x_{\alpha}, x_{-\alpha}\right\}$ e $\mathfrak{g}^{i}=\sum_{\alpha \in \Phi^{i} \cap-\Phi^{i}} \operatorname{span}\left\{h_{\alpha}, x_{\alpha}, x_{-\alpha}\right\}$ são álgebras de Lie semissimples, com sistemas de raízes $\Phi^{f} \cap-\Phi^{f}$ e $\Phi^{i} \cap-\Phi^{i}$, respectivamente.

Pelo Proposição 2.1.17,

$$
V^{\mathcal{N}_{+}} \cong W^{0} \otimes W^{f} \otimes W^{i},
$$

onde $W^{0}, W^{f}$ e $W^{i}$ são módulos de Harish-Chandra não nulos para as álgebras correntes $\mathfrak{z} \otimes A, \mathfrak{g}^{f} \otimes A$ e $\mathfrak{g}^{i} \otimes A$, respectivamente. Sendo assim, $W^{0}$ é unidimensional, pois $W^{0}$ possui dimensão finita e $\mathfrak{z} \otimes A$ é uma álgebra de Lie abeliana. Pelo primeiro item, $W^{f}$ é um $\mathfrak{g}^{f} \otimes A$ módulo de dimensão finita, pois $P\left(W^{f}\right)=\Phi^{f} \cap-\Phi^{f}$. Se $\mathfrak{g}^{i}=0$, então $W^{f}$ é o módulo trivial unidimensional. Se $\mathfrak{g}^{i}$ é simples, então, pelo segundo item, $W^{i}$ é um $\mathfrak{g}^{i} \otimes A$-módulo de dimensão infinita e uniformemente limitado. Suponha que $\mathfrak{g}^{i}$ é semissimples, então existem subsistemas de raízes dois a dois ortogonais $\Phi_{1}, \ldots, \Phi_{l} \subset \Phi^{i} \cap-\Phi^{i}$ e ideais $\mathfrak{g}_{1}, \ldots, \mathfrak{g}_{l}$ de $\mathfrak{g}^{i}$ que são álgebras de Lie simples com sistemas de raízes $\Phi_{1}, \ldots, \Phi_{r}$ e subálgebras de Cartan $\mathfrak{h}_{1}, \ldots, \mathfrak{h}_{r}$, respectivamente. Pelo Proposição 2.1.17, $W^{i} \cong \bigotimes_{j=1}^{l} W_{j}$, onde $W_{j}$ é um $\mathfrak{g}_{j}$-módulo de Harish-Chandra. Note que, para cada $j=1, \ldots, l, \alpha$ é uma raiz injetiva do $\mathfrak{g}_{j}$-módulo $W_{j}$ para todo $j=1, \ldots, l$. Para cada $j=1, \ldots, r$, seja $\lambda_{j} \in \mathfrak{h}_{j}^{*}$ um peso de $W_{j}$. Como $W_{j}$ é um $\mathfrak{g}_{j}$-módulo simples e todas as raízes de $\mathfrak{g}_{j}$ são injetivas em $W$, então, pelo segundo item deste teorema, os pesos de $W_{j}$ são exatamente os elementos do conjunto $\lambda_{j}+\sum_{\alpha \in \Phi_{j}} \mathbb{Z} \alpha$ e todos os espaços de peso possuem a mesma dimensão, que denotaremos por $m_{j}$. Note que podemos ver todos os pesos de $W_{j}$ e elementos do reticulado gerado por $\Phi_{j}$ como elementos de $\mathfrak{h}_{k}^{*}$ tomando-os como zero em $\mathfrak{h}_{k}$ para cada $k \neq j$. Portanto, $\lambda_{j}$ é um peso de $W_{k}$ se, e 
somente se, $\lambda_{j}=0$, implicando que $\lambda_{j}+n \alpha$ não é um peso de $W_{j}$ para todo $n \in \mathbb{Z}$ não nulo e $\alpha \in \Phi_{k}$, onde $k \neq j$. Sendo assim, os pesos de $W^{i}$ são da forma $\mu_{1}+\cdots+\mu_{l}$, onde $\mu_{j}$ é um peso de $W_{j}$ para cada $j=1, \ldots, l$, os espaços de peso de $W^{i}$ são exatamente os espaços $W_{\lambda_{1}+n_{1} \alpha_{1}+\cdots+\lambda_{l}+n \alpha_{l}}^{i}$, onde $n_{1}, \ldots, n_{l} \in \mathbb{Z}$ e $\alpha_{k} \in \Phi_{k}, k=1, \ldots, l$, e esse espaço é isomorfo a

$$
\bigotimes_{j=1}^{l}\left(W_{j}\right)_{\lambda_{j}+n_{j} \alpha_{j}} .
$$

Concluímos que $W^{i}$ é um $\mathfrak{g}^{i}$-módulo uniformemente limitado cujos espaços de peso possuem a mesma dimensão e esta é $m_{1} \cdots m_{l}$. Fazendo um argumento parecido, temo que $V^{\mathcal{N}_{+}} \cong$ $W^{0} \otimes W^{f} \otimes W^{i}$ é um $\mathcal{L}$-módulo uniformemente limitado.

Portanto, todo $\mathcal{G}$-módulo de Harish-Chandra simples será isomorfo ao módulo de indução parabólica $L_{\mathcal{P}}(W)$, onde $\mathcal{P}=\mathfrak{p} \otimes A$ é a subálgebra de $\mathcal{G}$ dada por $\mathfrak{p}=\mathfrak{h} \oplus \sum_{\alpha \in P} \mathfrak{g}_{\alpha}$ para algum subconjunto parabólico $P \subset \Phi$ e $W$ é um módulo uniformemente limitado sobre a álgebra de Lie $\mathcal{L}=\left(\mathfrak{h} \oplus \sum_{\alpha \in P \cap-P} \mathfrak{g}_{\alpha}\right) \otimes A$.

Observação 2.2.13. Dizemos que um $\mathcal{G}$-módulo de Harish-Chandra $V$ é um módulo integral se, dada uma base do sistema de raízes $\Delta \subset \Phi, x_{\alpha} \otimes 1$ e $x_{-\alpha} \otimes 1$ agem nilpotentemente em todo $V$ para todo $\alpha \in \Delta$. Pelo Lema 2.2.7, se $V$ é um $\mathcal{G}$-módulo de Harish-Chandra integral, então $\Phi^{f}(V)=\Phi$. Pelo Teorema 2.2.12, todo $\mathcal{G}$-módulo integral possui dimensão finita.

\subsection{Módulos de Avaliação}

Na prática, um módulo de avaliação nada mais é do que um $\mathcal{G}$-módulo criado a partir de $\mathfrak{g}$ módulos. Nesta seção, iremos definir o que é um módulo de avaliação, além de mostrar alguns resultados relacionados a ele.

Sejam $m_{1}, \ldots, m_{r} \subset A$ ideais maximais distintos e denote $\underline{m}=\left(m_{1}, \ldots, m_{r}\right)$. Como $A$ é uma álgebra finitamente gerada e $\mathbb{k}$ é algebricamente fechado, temos que $A / m_{i} \cong \mathbb{k}$, para todo $i=1, \ldots, r$. Portanto, para cada $a \in A$, podemos definir $a\left(m_{i}\right) \in \mathbb{k}$ tal que $a\left(m_{i}\right)+m_{i}=a+m_{i}$. Note que a aplicação $a \mapsto a\left(m_{i}\right)$ é um homomorfismo de k-álgebras, ou seja, é uma transformação linear tal que $(a b)\left(m_{i}\right)=a\left(m_{i}\right) b\left(m_{i}\right)$ para todo $a, b \in A$. Defina a aplicação de avaliação

$$
\begin{aligned}
e v \underline{m}: \mathfrak{g} \otimes A & \rightarrow \mathfrak{g}^{\oplus r} \\
x \otimes a & \mapsto\left(a\left(m_{1}\right) x, \ldots, a\left(m_{r}\right) x\right)
\end{aligned}
$$

Se $m_{1}, \ldots, m_{r}$ são distintos, pelo Teorema Chinês do Resto, $A /\left(m_{1} \ldots m_{r}\right) \cong A / m_{1} \times \cdots \times A / m_{r}$ e, portanto, a aplicação $a \mapsto\left(a\left(m_{1}\right), \ldots, a\left(m_{r}\right)\right)$ é sobrejetora. Logo, evm também é sobrejetor.

Sejam $W_{1}, \ldots, W_{r} \mathfrak{g}$-módulos, então $W_{1} \otimes \cdots \otimes W_{r}$ é um $\mathfrak{g}^{\oplus r}$-módulo e, portanto, o pullback de $e v \underline{m}$

$$
\mathfrak{g} \otimes A \stackrel{e v \underline{m}}{\longrightarrow} \mathfrak{g}^{\oplus r} \rightarrow \operatorname{End}\left(W_{1} \otimes \cdots \otimes W_{r}\right)
$$

define uma estrutura de $\mathfrak{g} \otimes A$-módulo em $W_{1} \otimes \cdots \otimes W_{r}$. A ação de $\mathfrak{g} \otimes A$ em $W_{1} \otimes \cdots \otimes W_{r}$ é dada por

$$
(x \otimes a)\left(w_{1} \otimes \cdots \otimes w_{r}\right)=\sum_{i=1}^{r} a\left(m_{i}\right) w_{1} \otimes \cdots \otimes\left(x w_{i}\right) \otimes \cdots \otimes w_{r} .
$$

Chamaremos tal módulo de módulo de avaliação e o denotaremos por

$$
V(\underline{m}, \underline{W})=W_{1}\left(m_{1}\right) \otimes \cdots \otimes W_{k}\left(m_{k}\right),
$$

onde $W_{i}\left(m_{i}\right)$ é o $\mathcal{G}$-módulo de avaliação dado por $(x \otimes a) w_{i}=a_{i}\left(m_{i}\right) x w_{i}$. 
Exemplo 2.3.1. Em [BLL15], um exemplo de $\mathcal{G}$-módulo de avaliação que não possui espaços de pesos de dimensão finita foi dado. Suponha $\mathbb{k}=\mathbb{C}$ e $\mathfrak{g}=\mathfrak{s l}_{2}(\mathbb{C})$, então $\mathfrak{s l}_{2}(\mathbb{C})$ possui base $e, f, h$, onde $[h, e]=2 e,[h, f]=-2 f$ e $[e, f]=h$. Seja $W$ o $\mathbb{C}$-espaço vetorial com base $\left\{v_{n} \mid n \in \mathbb{Z}\right\}$ e defina

$$
h v_{n}=2 i v_{n}, e v_{n}=-\frac{(2 n+1)^{2}}{4} v_{n+1}, \text { e } f v_{n}=v_{n-1}
$$

para todo $i \in \mathbb{Z}$. Estas relações tornam $W$ um $\mathfrak{s l}_{2}(\mathbb{C})$-módulo, pois

$$
\begin{aligned}
(h e-e h) v_{n} & =h\left(-\frac{(2 n+1)^{2}}{4} v_{n+1}\right)-2 n e v_{n} \\
= & \left(\frac{-(2 n+1)^{2}(2 n+2)+2 n(2 n+1)^{2}}{4}\right) v_{n+1} \\
= & \left(\frac{-4 n^{2}-4 n-1}{2}\right) v_{n+1} \\
= & \frac{(2 n+1)^{2}}{2} v_{n+1}=2 e v_{n}=[h, e] v_{n}, \\
(h f-f h) v_{n} & =h v_{n-1}-2 n f v_{n} \\
& =(2(n-1)-2 n) v_{n-1} \\
& =-2 v_{n-1}=-2 f v_{n}=[h, f] v_{n}, \\
(e f-f e) v_{n}= & e v_{n-1}+\frac{(2 n+1)^{2}}{4} f v_{n+1} \\
= & \frac{-4 n^{2}+4 n-1+4 n^{2}+4 n+1}{4} v_{n} \\
= & 2 n v_{n}=h v_{n}=[e, f] v_{n} .
\end{aligned}
$$

Os pesos de $W$ são dados por $2 n, n \in \mathbb{Z}$, e o espaço de peso $2 n$ é o subespaço vetorial de $W$ gerado por $\left\{v_{n}\right\}$. $W$ é um $\mathfrak{s l}_{2}(\mathbb{C})$-módulo de peso simples, pois se $U\left(\mathfrak{s l}_{2}(C)\right) v_{v}=W$, para todo $v \in \mathbb{Z}$.

Se $A$ é uma álgebra afim qualquer e $m_{1}, m_{2}$ são dois ideais maximais distintos, então o espaço de peso $(2 n+2 m)$ de $W\left(m_{1}\right) \otimes W\left(m_{2}\right)$ contém o conjunto linearmente independente infinito $\left\{v_{n-l} \otimes v_{m+l} \mid l \in \mathbb{Z}\right\}$, para todo $n, m \in \mathbb{Z}$. Logo, $W\left(m_{1}\right) \otimes W\left(m_{2}\right)$ não é um $\mathfrak{s l}_{2}(\mathbb{C}) \otimes A$-módulo quasi-finito.

Exemplo 2.3.2. Suponha que $A=\mathbb{k}[t]$, então, como $\mathbb{k}$ é algebricamente fechado, os ideais maximais de $A$ são da forma

$$
\langle t-\alpha\rangle
$$

onde $\alpha \in \mathbb{k}$ e $\langle t-\alpha\rangle$ é o ideal de $A$ gerado por $t-\alpha$. Se $\alpha \in \mathbb{k}$, então

$$
t+\langle t-\alpha\rangle=t-(t-\alpha)+\langle t-\alpha\rangle=\alpha+\langle t-\alpha\rangle \in \mathbb{k}[t] /\langle t-\alpha\rangle,
$$

portanto $t^{k}(\langle t-\alpha\rangle)=\alpha^{k}$ para cada $k \in \mathbb{Z}_{\geq 0}$. Sendo assim, para todo $f \in \mathbb{k}[t]$, temos $f(\langle t-\alpha\rangle)=$ $f(\alpha)$. Logo, se $V(\underline{m}, \underline{W})$ é um módulo de avaliação, então existem $\alpha_{1}, \ldots, \alpha_{r} \in \mathbb{k}$ tais que

$$
(x \otimes f) v_{1} \otimes \cdots \otimes v_{r}=\sum_{i=1}^{r} f\left(\alpha_{i}\right)\left(v_{1} \otimes \cdots \otimes\left(x v_{i}\right) \otimes \cdots \otimes v_{r}\right),
$$

para todo $f \in \mathbb{k}[t], x \in \mathfrak{g}$ e $v_{1} \otimes \cdots \otimes v_{r} \in V(\underline{m}, \underline{W})$.

Exemplo 2.3.3. Suponha que $A=\mathbb{k}\left[t, t^{-1}\right]$. Utilizaremos um pouco da teoria de localização para mostrar o que são os módulos de avaliação de $A$, para mais informações veja o Capítulo 4 de [BT15] 
ou o Chapter 3 de [AM69]. Como $\mathbb{k}[t]$ é domínio de ideais principais, todo ideal primo é maximal. Além disso, $\mathbb{k}\left[t, t^{-1}\right]=S^{-1} \mathbb{k}[t]$, onde $S=\left\{t^{k} \mid k \in \mathbb{Z}_{\geq 0}\right\}$, portanto os ideais maximais de $\mathbb{k}\left[t, t^{-1}\right]$ são da forma $S^{-1} m$ tal que $m$ é um ideal maximal de $\mathbb{k}[t]$ e $t \notin m$. Logo, os ideais maximais de $\mathbb{k}\left[t, t^{-1}\right]$ são da forma

$$
\langle t-\alpha\rangle
$$

onde $\alpha \in \mathbb{k}, \alpha \neq 0$ e $\langle t-\alpha\rangle$ é o ideal de $\mathbb{k}\left[t, t^{-1}\right]$ gerado por $t-\alpha$. Como feito no Exemplo 2.3.2, $t^{k}(\langle t-\alpha\rangle)=\alpha^{k}$ para cada $k \in \mathbb{Z}_{\geq 0}$. Além disso,

$$
\langle t-\alpha\rangle=t^{-1}(t-\alpha)+\langle t-\alpha\rangle=1-\alpha t^{-1}+\langle t-\alpha\rangle \Longrightarrow t^{-1}+\langle t-\alpha\rangle=\alpha^{-1}+\langle t-\alpha\rangle .
$$

Sendo assim, $t^{k}(\langle t-\alpha\rangle)=\alpha^{k}$ para todo $k \in \mathbb{Z}$, implicando que, para todo $f \in \mathbb{k}[t], f(\langle t-\alpha\rangle)=f(\alpha)$. Logo, se $V(\underline{m}, \underline{W})$ é um módulo de avaliação, então existem $\alpha_{1}, \ldots, \alpha_{r} \in \mathbb{k}$ todos não nulos tais que

$$
(x \otimes f) v_{1} \otimes \cdots \otimes v_{r}=\sum_{i=1}^{r} f\left(\alpha_{i}\right)\left(v_{1} \otimes \cdots \otimes\left(x v_{i}\right) \otimes \cdots \otimes v_{r}\right),
$$

para todo $f \in \mathbb{k}[t], x \in \mathfrak{g}$ e $v_{1} \otimes \cdots \otimes v_{r} \in V(\underline{m}, \underline{W})$.

Exemplo 2.3.4. Seja $k>1$ e suponha $A=\mathbb{k}[t] /\left\langle t^{k}\right\rangle$. Pelo Teorema de Correspondência, todos os ideais de $A$ são da forma $I /\left\langle t^{k}\right\rangle$, onde $I$ é um ideal de $\mathbb{k}[t]$ que contém $\left\langle t^{k}\right\rangle$. Logo, $A$ possui um único ideal maximal e este é o ideal gerado por $t+\left\langle t^{k}\right\rangle$. Sendo assim, se $V(\underline{m}, \underline{W})$ é um $\mathcal{G}$-módulo de avaliação, então $V(\underline{m}, \underline{W})=W$, onde $W$ é um $\mathfrak{g}$-módulo e

$$
\left(x \otimes\left(f+\left\langle t^{k}\right\rangle\right)\right) w=f(0) x w,
$$

para todo $f \in \mathbb{k}[t], x \in \mathfrak{g}$ e $w \in W$.

Antes de avançarmos, recomendo o leitor à voltar à Proposição 1.3.22 e a Seção 1.3.2, onde provamos resultados relacionados ao produto tensorial de módulos.

Proposição 2.3.5. Se $W_{1}, \ldots, W_{r}$ são g-módulos simples e $m_{1}, \ldots, m_{r} \subset A$ são ideais maximais distintos entre si, então $V(\underline{M}, \underline{W})$ é um $\mathcal{G}$-módulo simples.

Demonstração. Utilizando indução e o Proposição 1.3 .22 , segue que $W_{1} \otimes \cdots \otimes W_{r}$ é um $\mathfrak{g}^{\oplus r}$-módulo simples. Seja $\varphi: \mathfrak{g}^{\oplus r} \rightarrow W_{1} \otimes \cdots \otimes W_{r}$ a representação associada.

Como os ideais maximais são distintos, pelo Teorema Chinês do Resto (Teorema 1.1.13), evm : $\mathfrak{g} \otimes A \rightarrow \mathfrak{g}^{\oplus r}$ é sobrejetor. Isto é, existem $a_{1}, \ldots a_{r} \in A$ tais que $a_{i}\left(m_{j}\right)=\delta_{i j}$ e, para todo $x \in \mathfrak{g}$,

$$
\left(x \oplus a_{i}\right)\left(w_{1} \otimes w_{r}\right)=w_{1} \otimes \cdots \otimes x w_{i} \otimes \ldots w_{r}, \quad \text { para todo } i=1, \ldots, r .
$$

Logo, $e v \underline{m} \circ \varphi$ é uma representação de $\mathfrak{g} \otimes A$ simples.

Teorema 2.3.6 ( [BLL15, Theorem 3.2]). Seja $\mathfrak{g}$ uma álgebra de Lie simples. Seja $V$ um módulo de Harish-Chandra sobre $\mathcal{G}$, com representação associada $\phi: \mathfrak{g} \otimes A \rightarrow$ End $V$. Suponha que exista $m_{1}, \ldots, m_{r} \in$ Specm $A$ e um morfismo de álgebras de Lie $\psi: \mathfrak{g}^{\oplus r} \rightarrow$ End $V$ tal que $\phi=\psi \circ$ evm. Então, $V$ é isomorfo a um módulo de avaliação de $\mathcal{G}$.

Demonstração. Como $m_{1}, \ldots, m_{r}$ são distintos, pelo Teorema Chinês do Resto (Teorema 1.1.13), existem

$$
a_{1}, \ldots, a_{r} \in A \text { tais que } a_{i}\left(m_{j}\right)=\delta_{i j}
$$

para todo $i, j=1, \ldots, r$. Denote por $\mathcal{H}$ a subálgebra de Lie de $\mathfrak{g} \otimes A$ abeliana de dimensão finita $\mathfrak{h} \otimes \operatorname{span}_{\mathfrak{k}}\left\{s_{1}, \ldots, s_{r}\right\}$. Seja $\lambda \in \Pi(V)$. Como $\mathcal{H}$ comuta com $\mathfrak{h} \otimes 1$ e $V_{\lambda}$ possui dimensão finita, então, pelo Teorema de Lie (Teorema 1.2.8), existe $v \in V_{\lambda}$ não nulo tal que $v$ é um autovetor para todo elemento em $\mathcal{H}$. 
Note que $\operatorname{evm}\left(\mathfrak{g} \otimes\left\langle a_{i}\right\rangle\right)=0 \oplus \cdots \oplus \mathfrak{g} \oplus \cdots \oplus 0$, onde $\mathfrak{g}$ é o $i$-ésimo somando no lado direito da igualdade. $\operatorname{Logo}, e v \underline{m}(\mathcal{H})=\mathfrak{h}^{\oplus r}$ é a álgebra de Cartan de $\mathfrak{g}^{\oplus r}$ e $v \in V$ é um autovetor para a ação de $\mathfrak{h}^{\oplus r}$ em $V_{\lambda}$. Então $U\left(\mathfrak{g}^{\oplus r}\right) v$ é um $\mathfrak{g}^{\oplus r}$ submódulo não nulo do módulo simples $V$. Logo, $V=U\left(\mathfrak{g}^{\oplus r}\right) v$ é um $\mathfrak{g}^{\oplus r}$-módulo de peso simples, com relação a subálgebra de Cartan $\mathfrak{h}^{\oplus r}$. Pelo Corolário 1.3.36, segue que $V \cong V_{1} \otimes \cdots \otimes V_{r}$, onde $V_{1}, \ldots, V_{r}$ são $\mathfrak{g}$-módulos de peso simples.

Seja $\mathcal{W}$ o conjunto de todas classes de isomorfismo de $(\mathfrak{g}, \mathfrak{h})$-módulos de peso simples. Para cada $W$ g-módulo de peso simples, denote $[W]$ como sendo a classe de isomorfismo de $W$ em $\mathcal{W}$. Em particular, denotaremos por $[\mathbb{k}]$ a classe do $\mathfrak{g}$-módulo trivial unidimensional.

Lema 2.3.7. Se $\rho: \mathcal{G} \rightarrow \operatorname{End}(V(\underline{M}, \underline{W}))$ é uma representação de avaliação que é um módulo de Harish-Chandra, então $\operatorname{ker}(\rho)=\mathfrak{g} \otimes \bigcap_{i=1}^{r} M_{i}$.

Demonstração. Se $r>1$ e $W_{i} \in[\mathbb{k}]$, para algum $i=1, \ldots, r$, então $V(\underline{m}, \underline{W})$ é isomorfo a

$$
V\left(\left(m_{1}, \ldots, m_{i-1}, m_{i+1}, \ldots, m_{r}\right),\left(W_{1}, \ldots, W_{i-1}, W_{i+1}, \ldots, W_{r}\right) .\right.
$$

Sendo assim, podemos supor $W_{i} \notin[\mathbb{k}]$ para todo $i=1, \ldots, r$.

Seja $\phi: \mathfrak{g}^{\oplus r} \rightarrow W_{1} \otimes \cdots \otimes W_{r}$ tal que $\rho=\phi \circ$ ev $\underline{m}, \underline{W}$. Se $x \otimes a \in \mathfrak{g} \otimes \bigcap_{i=1}^{r} m_{i}$, então $a\left(m_{i}\right)=0$, para cada $i=1, \ldots, r$. Logo, evm, $\underline{W}(x \otimes a)=(0, \ldots, 0)$ e, assim, $\rho(x \otimes a)=0$. Portanto, $\mathfrak{g} \otimes \bigcap_{i=1}^{r} m_{i} \subset \operatorname{ker}(\rho)$.

Provaremos que $\operatorname{ker}(\rho) \subset \mathfrak{g} \otimes \bigcap_{j=1}^{r} m_{j}$. Pela Proposição 2.1.11, $\rho=\mathfrak{g} \otimes I$, onde $I$ é um ideal de A. Seja

$$
\sum_{i=1}^{l} x_{i} \otimes a_{i} \in \operatorname{ker}(\rho)
$$

tal que $a_{1}, \ldots, a_{l} \in I$ e $\left\{x_{i}\right\}_{i=1}^{l} \subset \mathfrak{g}$ é um conjunto linearmente independente. Se $a_{i}\left(m_{j}\right)=0$ para todo $j \in\{1, \ldots, r\}$ e para todo $i \in\{1, \ldots, l\}$, então $a_{i} \in \bigcap_{j=1}^{l} m_{j}$ para cada $i=1, \ldots, l$. Suponha, sem perda de generalidade, que $a_{1} \notin m_{s}$ para algum $s \in\{1, \ldots, r\}$, isto é, $a_{1}\left(m_{s}\right) \neq 0$. Fixe tal $s \in\{1, \ldots, r\}$.

Seja $0 \neq w_{1} \otimes \cdots \otimes w_{r} \in V(\underline{m}, \underline{W})$, então

$$
\begin{aligned}
\left(\sum_{i=1}^{l} x_{i} \otimes a_{i}\right) w_{1} \otimes \cdots \otimes w_{r} & =\sum_{i=1}^{l} \sum_{j=1}^{r} a_{i}\left(m_{j}\right) w_{1} \otimes \cdots \otimes x_{i} w_{j} \otimes \cdots \otimes w_{r} \\
& =\sum_{j=1}^{r} w_{1} \otimes \cdots \otimes\left(\sum_{i=1}^{l} a_{i}\left(m_{j}\right) x_{i} w_{j}\right) \otimes \cdots \otimes w_{r} \\
& =0
\end{aligned}
$$

Como $w_{1} \otimes \cdots \otimes w_{r} \neq 0$, temos que $\sum_{i=1}^{l} a_{i}\left(m_{j}\right) x_{i} w_{j}=0$ para todo $j=1, \ldots, r$. Usando o mesmo argumento, se $u \in U(\mathfrak{g})$ é tal que $u w_{s} \neq 0$, então $\sum_{i=1}^{l}\left(a_{i}\left(m_{s}\right) x_{i}\right) u w_{s}=0$ também.

Como $\mathfrak{g}$ é simples, a representação adjunta torna $\mathfrak{g}$ um $\mathfrak{g}$-módulo simples de dimensão finita. Pelo Teorema 1.3.21, existe $u \in U(\mathfrak{g})$ tal que $\operatorname{ad}_{u}\left(x_{i}\right)=\delta_{1 i} x_{1}$ para todo $i=1, \ldots, l$. Logo,

$$
\begin{aligned}
0 & =u\left(\sum_{i=1}^{l} a_{i}\left(m_{s}\right) x_{i}\right) w_{s} \\
& =\left(\sum_{i=1}^{l} a_{i}\left(m_{s}\right) x_{i}\right) u w_{s}+\operatorname{ad}_{u}\left(\sum_{i=1}^{l} a_{i}\left(m_{s}\right) x_{s}\right) w_{s} \\
& =a_{1}\left(m_{s}\right) x_{1} w_{s}
\end{aligned}
$$


pelo argumento dado acima de que $\left(\sum_{i=1}^{l} a_{i}\left(m_{s}\right) x_{i}\right) u w_{s}=0$ se $u w_{s} \neq 0$. Daí teríamos que $a\left(m_{s}\right) x_{1} w_{s}=0$. Como $\left\{x_{1}, \ldots, x_{l}\right\} \subset \mathfrak{g}$ é linearmente independente, $x_{1}$ é não nulo. Como $W_{s}$ é simples e não está em $[\mathbb{k}]$, existe $w_{s} \in W_{s}$ tal que $x_{1} w_{s} \neq 0$. Sendo assim, $a_{1}\left(m_{s}\right) x_{1} w_{s}=0$ implica que $a_{1}\left(m_{s}\right)=0$, contradizendo a hipótese de que $a_{1}\left(m_{s}\right) \neq 0$. Logo, não existe $i \in\{1, \ldots, l\}$ tal que $a_{i}\left(m_{j}\right)$ para algum $j \in\{1, \ldots, r\}$, então $a_{i} \in \bigcap_{j=1}^{r} m_{j}$ para todo $i=1, \ldots, l$. Segue que ker $\rho \subset \mathfrak{g} \otimes \bigcap_{j=1}^{r} m_{j}$.

Teorema 2.3.8. Sejam $m_{1}, \ldots, m_{r}, n_{1}, \ldots, n_{s} \subset A, m_{i} \neq m_{j}, n_{i} \neq n_{j}$, se $i, \neq j$, ideais maximais de $A$ e $W_{1}, \ldots, W_{r}, U_{1}, \ldots, U_{s} \mathfrak{g}$ módulos de Harish-Chandra tais que $V(\underline{m}, \underline{W})$ e $V(\underline{n}, \underline{U})$ são módulos de Harish-Chandra isomorfos, onde $\underline{m}=\left(m_{1}, \ldots, m_{r}\right), \underline{n}=\left(n_{1}, \ldots, n_{s}\right), \underline{W}=$ $\left(W_{1}, \ldots, W_{r}\right), \underline{U}=\left(U_{1}, \ldots, U_{r}\right)$. Então $r=s$ e existe uma permutação $\sigma$ de $\{1, \ldots, r\}$ tal que $N_{i}=m_{\sigma(i)}$ e $U_{i} \cong W_{\sigma(i)}$.

Demonstração. Sejam $\alpha: \mathfrak{g} \otimes A \rightarrow V(\underline{m}, \underline{W})$ e $\beta: \mathfrak{g} \otimes A \rightarrow V(\underline{n}, \underline{U})$ as representações associadas e suponha que não sejam a representação trivial unidimensional. Logo, ker $\alpha=\operatorname{ker} \beta$ e, pelo Lema 2.3.7,

$$
\mathfrak{g} \otimes \bigcap_{i=1}^{r} m_{r}=\mathfrak{g} \otimes \bigcap_{i=1}^{s} n_{i} .
$$

Logo, $\bigcap_{i=1}^{r} m_{r}=\bigcap_{i=1}^{s} n_{i}$. Como $m_{i} \neq m_{j}$ e $n_{i} \neq n_{j}$, se $i \neq j$, então, pelo Teorema Chinês do Resto e Teorema dos Zeros de Hilbert, $r=s$ e existe uma permutação $\sigma$ de $\{1, \ldots, r\}$ tal que $n_{i}=m_{\sigma(i)}$.

Note que a aplicação linear $U_{1} \otimes \cdots \otimes U_{r} \rightarrow U_{\sigma(1)} \otimes \cdots \otimes U_{\sigma(r)}$ dada por $u_{1} \otimes \cdots \otimes u_{r} \mapsto$ $u_{\sigma(1)} \otimes \cdots \otimes u_{\sigma(r)}$ é um isomorfismo de $\mathfrak{g} \otimes A$-módulos. Logo, podemos supor, sem perda de generalidade, que $\sigma$ é a permutação identidade.

Sejam $a_{1}, \ldots, a_{r} \in A$ tais que $a_{i}\left(m_{j}\right)=\delta_{i j}$, então

$$
\alpha\left(h \otimes a_{i}\right) u_{1} \otimes \cdots \otimes u_{r}=u_{1} \otimes \cdots \otimes h u_{i} \otimes \cdots \otimes u_{r} .
$$

Seja $u=\left(u_{1} \otimes \cdots \otimes u_{r}\right) \in V(\underline{n}, \underline{U})$ não nulo. Seja $\varphi: V(\underline{n}, \underline{U}) \rightarrow V(\underline{m}, \underline{W})$ um isomorfismo de $\mathcal{G}$-módulos e $w=\sum_{j=1}^{l} w_{1}^{j} \otimes \cdots \otimes w_{r}^{j} \in V(\underline{m}, \underline{W})$ tal que $\varphi(u)=w$ e $w_{1}^{j} \otimes \cdots \otimes w_{r}^{j} \neq 0$ para todo $j=1, \ldots, l]$. Fixe $i \in\{1, \ldots, r\}$. Se $f \in U(\mathfrak{g})$, então, pelo Teorema de Poincaré-Birkhoff-Witt, $f$ é uma combinação linear da forma

$$
f=\sum_{\substack{\alpha_{1}, \ldots, \alpha_{p} \in \Phi \\ \beta_{1}, \ldots, \beta_{q} \in \Delta}} c_{\alpha_{1}, \ldots, \alpha_{p}}^{\beta_{1}, \ldots, \beta_{q}} x_{\alpha_{1}} \ldots x_{\alpha_{p}} h_{\beta_{1}} \ldots h_{\beta_{q}}
$$

onde $\Delta \subset \Phi$ é um sistema de raízes simples. Sendo assim, para cada $f \in U(\mathfrak{g})$, defina $\tilde{f} \in U(\mathcal{G})$ por

$$
\tilde{f}=\sum_{\substack{\alpha_{1}, \ldots, \alpha_{p} \in \Phi \\ \beta_{1}, \ldots, \beta_{q} \in \Delta}} c_{\alpha_{1}, \ldots, \alpha_{p}}^{\beta_{1}, \ldots, \beta_{q}}\left(x_{\alpha_{1}} \otimes a_{i}\right) \ldots\left(x_{\alpha_{p}} \otimes a_{i}\right)\left(h_{\beta_{1}} \otimes a_{i}\right) \ldots\left(h_{\beta_{q}} \otimes a_{i}\right) .
$$

Sem perda de generalidade, suponha que $\left\{w_{i}^{j}\right\}_{j=1}^{l}$ seja um conjunto linearmente independente em $W_{i}$. Pelo Teorema 1.3.21, existe $f_{i} \in U(\mathfrak{g})$ tal que $f_{i} w_{i}^{j}=\delta_{1 j} w_{1}^{j}$. Como $\varphi\left(\alpha\left(\tilde{f}_{i}\right) u\right)=\beta\left(\tilde{f}_{i}\right) w$, temos que

$$
\begin{aligned}
\varphi\left(u_{1} \otimes \cdots \otimes f_{i} u_{i} \otimes \cdots \otimes u_{r}\right) & =\varphi\left(\alpha\left(\tilde{f}_{i}\right) u\right) \\
& =\beta\left(\tilde{f}_{i}\right) w \\
& =\sum_{j=1}^{l} w_{1}^{j} \otimes \otimes \cdots \otimes f_{i} w_{i}^{j} \otimes \cdots \otimes w_{r}^{j} \\
& =w_{1}^{1} \otimes \otimes \cdots \otimes w_{i}^{1} \otimes \cdots \otimes w_{r}^{1} .
\end{aligned}
$$


Como $w_{1}^{1} \otimes \cdots \otimes w_{i}^{1} \otimes \cdots \otimes w_{r}^{1}$ não é nulo e $\varphi$ é isomorfismo, temos que $f_{i} u_{i}$ não é nulo. Como $W_{i}$ e $U_{i}$ são $\mathfrak{g}$-módulos simples, $W_{i}=U(\mathfrak{g}) w_{i}^{1}$ e $U_{i}=U(\mathfrak{g}) f_{i} u_{i}$. Além disso, as aplicações

$$
\begin{gathered}
g\left(u_{1} \otimes \cdots \otimes f_{i} u_{i} \otimes \cdots \otimes u_{r}\right) \mapsto \alpha(\tilde{g}) u_{1} \otimes \cdots \otimes f_{i} u_{i} \otimes \cdots \otimes u_{r}=u_{1} \otimes \cdots \otimes g f_{i} u_{i} \otimes \cdots \otimes u_{r} \\
g\left(w_{i}^{1} \otimes \cdots \otimes w_{r}^{1} \mapsto \beta(\tilde{g}) w_{i}^{1} \otimes \cdots \otimes w_{r}^{1}=w_{i}^{1} \otimes \cdots \otimes g w_{i}^{1} \otimes \cdots \otimes w_{r}^{1}\right.
\end{gathered}
$$

definem uma estrutura de $\mathfrak{g}$-módulo em $\mathbb{k} u_{1} \otimes \cdots \otimes U_{i} \otimes \cdots \otimes \mathbb{k} u_{r}$ e $\mathbb{k} w_{1}^{1} \otimes \cdots \otimes W_{i} \otimes \cdots \otimes \mathbb{k} w_{1}^{r}$, respectivamente. Portanto, temos que, como g-módulos, $U_{i} \cong \mathbb{k} u_{1} \otimes \cdots \otimes U_{i} \otimes \cdots \otimes \mathbb{k} u_{r}$ e $W_{i} \cong$ $\mathbb{k} w_{1}^{1} \otimes \cdots \otimes W_{i} \otimes \cdots \otimes \mathbb{k} w_{1}^{r}$. Como $\varphi$ define um isomorfismo de $\mathfrak{g}$-módulos entre $\mathbb{k} u_{1} \otimes \cdots \otimes U_{i} \otimes \cdots \otimes \mathbb{k} u_{r}$ e $\mathbb{k} w_{1}^{1} \otimes \cdots \otimes W_{i} \otimes \cdots \otimes \mathbb{k} w_{1}^{r}$, então a composição

$$
U_{i} \cong \mathbb{\rightrightarrows} \mathbb{k} u_{1} \otimes \cdots \otimes U_{i} \otimes \cdots \otimes \mathbb{k} u_{r} \stackrel{\varphi}{\rightarrow} \mathbb{k} w_{1}^{1} \otimes \cdots \otimes W_{i} \otimes \cdots \otimes \mathbb{k} w_{1}^{r} \stackrel{\cong}{\rightarrow} W_{i}
$$

define um isomorfismo de $\mathfrak{g}$-módulos entre $U_{i}$ e $W_{i}$.

Para cada $m_{1}, \ldots, m_{r} \subset A$ ideais maximais distintos de $A$ e $W_{1}, \ldots, W_{r} \mathfrak{g}$-módulos de peso simples, defina $\Phi_{\underline{m}, \underline{W}}:$ Specm $A \rightarrow \mathcal{W}$ por

$$
\Phi_{\underline{m}, \underline{W}}(n)= \begin{cases}{\left[W_{i}\right]} & \text { se } n=m_{i}, \\ {[\mathbb{k}]} & \text { caso contrário, }\end{cases}
$$

onde $\left[W_{i}\right]$ e [k] é classe de isomorfismo de $\mathfrak{g}$-módulos de $W_{i}$ e do $\mathfrak{g}$-módulo unidimensional trivial, respectivamente. Para cada função $\Phi:$ Specm $A \rightarrow \mathcal{W}$, definimos o suporte de $\Phi$ supp $\Phi$ por

$$
\text { supp } \Phi=\{m \in \operatorname{Specm} A \mid \Phi(m) \neq[k]\} .
$$

As funções $\Phi \underline{m}, \underline{W}$ definidas como acima sempre possuem suporte finito.

Para cada $m_{1}, \ldots, m_{r} \subset A$ ideais maximais distintos e $W_{1}, \ldots, W_{r} \mathfrak{g}$-módulos de peso simples, podemos associar $V(\underline{m}, \underline{W})$ a função $\Phi \underline{m}, \underline{W}$. A correspondência que associa o $\mathfrak{g}$-módulos de peso de avaliação simples $V(\underline{m}, \underline{W})$ às aplicações de suporte finito $\Phi_{\underline{m}, \underline{W}}$ é sobrejetor. Pelo Teorema 2.3.8, a aplicação sobrejetora $[V(\underline{m}, \underline{W})] \mapsto \Phi \underline{m}, \underline{W}$ das classes de isomorfismo de módulos de avaliação simples para funções Specm $A \rightarrow \mathcal{W}$ com suporte finito está bem definida e é injetora. Em outras palavras:

Teorema 2.3.9 ( [BLL15, Theorem 3.6]). Existe uma correspondência biunívoca entre as classes de isomorfismo de $\mathcal{G}$-módulos de avaliação de Harish-Chandra e as funções $\Phi: \operatorname{Specm} A \rightarrow \mathcal{W}$ de suporte finito. Explicitamente, a classe de isomorfismo de $V(\underline{m}, \underline{W})$ corresponde à função $\Phi_{\underline{m}, \underline{W}}$.

\subsection{Módulos Uniformemente Limitados Simples}

Pelo Teorema 2.2.12, todo $\mathcal{G}$-módulo de Harish-Chandra ou é uniformemente limitado ou é indução parabólica de um módulo uniformemente limitado sobre uma álgebra corrente. O objetivo desta seção é dar uma classificação para os $\mathcal{G}$-módulos de Harish-Chandra uniformemente limitados. Primeiramente, mostraremos quando módulos de avaliação são módulos de Harish-Chandra uniformemente limitados. Em seguida, investigaremos o núcleo da representação associada a um módulo de Harish-Chandra uniformemente limitado. Por fim, mostraremos que todo $\mathcal{G}$-módulo de Harish-Chandra uniformemente limitado é um módulo de avaliação.

Lema 2.4.1. Sejam $W_{1}$ e $W_{2} \mathfrak{g}$-módulos de peso simples uniformemente limitados de dimensão infinita. Se $\Phi_{1}^{i}=\Phi^{i}\left(W_{1}\right)$ e $\Phi_{2}^{i}=\Phi^{i}\left(W_{2}\right)$ são os conjuntos das raízes injetivas em $W_{1}$ e $W_{2}$, respectivamente, e são tais que $\Phi_{1}^{i} \cap \Phi_{2}^{i} \neq \emptyset$, então $W_{1} \otimes W_{2}$ não é um $\mathfrak{g}$-módulo uniformemente limitado.

Demonstração. Sejam $W_{1, \lambda} \subset W_{1}$ e $W_{2, \mu} \subset W_{2}$ dois subespaços de peso de $W_{1}$ e $W_{2}$, respectivamente. Suponha $\Phi_{1}^{i} \cap-\Phi_{2}^{i} \neq \emptyset$ e tome $\alpha \in \Phi_{1}^{i} \cap-\Phi_{2}^{i}$. Logo, $W_{1, \lambda+n \alpha} \neq 0$ e $W_{2, \mu-n \alpha} \neq 0$ para todo 
$n \in \mathbb{Z}_{\geq 0}$. Sendo assim, o subespaço de $W_{1} \otimes W_{2}$ de peso $\lambda+\mu$ contém o subespaço de dimensão infinita

$$
\sum_{n=0}^{\infty} W_{1, \lambda+n \alpha} \otimes W_{2, \mu-n \alpha}
$$

então $W_{1} \otimes W_{2}$ não é um módulo quasi-finito, portanto não é uniformemente limitado.

Suponha que $\Phi_{1}^{i} \cap-\Phi_{2}^{i} \neq \emptyset$ e fixe $\alpha \in \Phi_{1}^{i} \cap \Phi_{2}^{i}$, então $W_{1, \lambda+l \alpha} \neq 0$ e $W_{2, \mu+(n-l) \alpha} \neq 0$ para todo $n \in \mathbb{Z}_{\geq 0}$. Portanto o subespaço de $W_{1} \otimes W_{2}$ de peso $\lambda+\mu+n \alpha$ contém o subespaço que possui dimensão maior ou igual a $n+1$ dado pela soma

$$
\sum_{l=0}^{n} W_{1, \lambda+l \alpha} \otimes W_{2, \mu+(n-l) \alpha} .
$$

Como isto vale para todo $n \in Z_{>0}$, não existe um majorante para as dimensões do espaços de peso $W_{1} \otimes W_{2}$, então $W_{1} \otimes W_{2}$ não é uniformemente limitado.

Para uma base $\Delta \subset \Phi$ e um subconjunto $X \subset \Phi$, denotamos por $\Phi_{+}(\Delta)$ o conjunto das raízes positivas em relação à base $\Delta$ e por $X^{+}=X \cap \Phi_{+}(\Delta)$. Além disso, dizemos que $X \subset X^{\prime}$ é um ideal de $X \subset \Phi$, se para todo $\alpha \in X$ e $\beta \in X^{\prime}$ tal que $\alpha+\beta \in \Phi$, temos que $\alpha+\beta \in X$. Para uma raiz $\alpha \in \Phi$, denotaremos por $\langle\alpha\rangle(X)$ o menor ideal de $X \subset \Phi$ que contém $\alpha$. Em particular, se $\Delta$ é uma base de raízes simples em $\Phi$ e $\alpha \in \Delta$, então

$$
\langle-\alpha\rangle\left(\Phi_{-}(\Delta)\right)=\left\{-\alpha-\mu \mid \mu \in \Phi_{+}(\Delta) \cup\{0\} \text { tal que }-\alpha-\mu \in \Phi\right\},
$$

pois $-\alpha+\mu \in\langle-\alpha\rangle\left(\Phi_{-}(\Delta)\right)$ se, e somente se, $\mu \in \Phi_{-}(\Delta)$ ou $\mu=0$ é tal que $-\alpha+\mu \in \Phi$.

Lema 2.4.2. Seja $\alpha \in \Delta$, onde $\Delta$ é uma base de raízes simples em $\Phi$. Se $\beta \in \Phi \backslash \pm\langle-\alpha\rangle$, então existe $\gamma \in\langle-\alpha\rangle$ tal que $\beta+\gamma \in\langle-\alpha\rangle$.

Demonstração. Para todo $\lambda \in \sum_{\tau \in \Delta} \mathbb{Z} \tau$ no reticulado de raízes gerado por $\Phi$, escreva

$$
\lambda=\sum_{\tau \in \Delta} \eta_{\tau}^{\lambda} \tau
$$

a combinação $\mathbb{Z}$-linear de $\lambda$ em relação à $\Delta$. Em particular, se $\tau \in \Phi$, teremos que $\eta_{\tau}^{\gamma} \leq 0$ ou $\eta_{\beta}^{\gamma} \geq 0$ para todo $\tau \in \Delta$. Seja $\theta \in \Phi$ uma raiz de altura máxima - isto é, $\theta$ é um elemento de $\Phi$ tal que $\eta_{\tau}^{\theta} \geq 1$ para todo $\tau \in \Delta$ e $\sum_{\tau \in \Delta} \eta_{\tau}^{\theta} \geq \sum_{\tau \in \Delta} \eta_{\tau}^{\lambda}$ para todo $\lambda \in \Phi$. Como g é simples e $\left\{x_{\tau}, x_{-\tau} \mid \tau \in \Delta\right\}$ gera $\mathfrak{g}$ como álgebra de Lie, existem $x_{1}, \ldots, x_{l} \in\left\{x_{\tau}, x_{-\tau} \mid \tau \in \Delta\right\}$ tais que $\operatorname{ad}_{x_{l}} \ldots \operatorname{ad}_{x_{1}}\left(x_{\alpha}\right) \in \mathfrak{g}_{\theta}$. Como $\theta \in \Phi_{+}(\Delta)$, podemos supor que $x_{1}, \ldots, x_{l} \in\left\{x_{\tau} \mid \tau \in \Delta\right\}$. Sendo assim, se $\mu_{0}=\alpha$ e $\mu_{i} \in \Phi$ é tal que $\operatorname{ad}_{x_{i}} \ldots \operatorname{ad}_{x_{1}}\left(x_{\alpha}\right) \in \mathfrak{g}_{\mu_{i}}, i=1, \ldots, l$, teremos que

$$
\alpha=\mu_{0} \prec \mu_{1} \prec \cdots \prec \mu_{r}=\theta,
$$

onde $\prec$ é a ordem parcial que $\Delta$ induz em $\Phi$. Além disso, $\mu_{i}-\mu_{i-1} \in \Delta$ para todo $i=1, \ldots, l$, se definirmos $\mu_{-1}=0$.

Seja $\beta \in \Phi \backslash \pm\langle-\alpha\rangle\left(\Phi_{-}(\Delta)\right)$. Como a forma de Killing $\kappa(-,-): \mathfrak{h}^{*} \times \mathfrak{h}^{*} \rightarrow \mathbb{k}$ é não degenerada e toda rais da base $\Delta$ aparece em $\mu_{i}-\mu_{i-1}$ para algum $i \geq 0$, teremos que $\kappa\left(\beta, \mu_{s}\right) \neq 0$ para algum $0 \leq s \leq l$. Portanto, $\beta+\mu_{s}$ ou $\beta-\mu_{s}$ é uma raiz (veja Lema 1.2.29).

Seja $\alpha \in \Delta$. Utilizando um argumento parecido ao dado no primeiro parágrafo e que $-\alpha+\mu \in$ $\langle-\alpha\rangle\left(\Phi_{-}(\Delta)\right)$ se, e somente se, $\mu \in \Phi_{-}(\Delta)$ ou $\mu=0$ é tal que $-\alpha+\mu \in \Phi$, temos que

$$
\langle-\alpha\rangle\left(\Phi_{-}(\Delta)\right)=\left\{\lambda \in \Phi \mid \eta_{\alpha}^{\lambda}<0\right\} .
$$

Assim, $\eta_{\alpha}^{\beta}=0$, pois $\beta \notin \pm\langle-\alpha\rangle$. Além disso, $\eta_{\alpha}^{\beta+\mu_{s}}>0$ e $\eta_{\alpha}^{\beta-\mu_{s}}<0$, pois $\eta_{\alpha}^{\mu_{s}} \neq 0$. Se $\lambda \in \Phi$, toda os coeficientes não nulos $\eta_{\tau}^{\lambda}$ terão que ser apenas positivos ou apenas negativos. Portanto se $\beta+\mu_{s}$ é uma raiz, segue que $\beta+\mu_{s} \in \Phi_{+}(\Delta)$. Por outro lado, se $\beta-\mu_{s}$, teremos que $\beta-\mu_{s} \in \Phi_{-}(\Delta)$. 
Se $\beta+\mu_{s} \in \Phi_{+}(\Delta)$, então $-\beta-\mu_{s} \in \Phi_{-}(\Delta)$ e $\eta_{\alpha}^{-\beta-\mu_{s}}<0$, então $-\beta-\mu_{s} \in\langle-\alpha\rangle\left(\Phi_{-}(\Delta)\right)$. Assim, $-\beta-\mu_{s} \in\langle-\alpha\rangle\left(\Phi_{-}(\Delta)\right)$ é tal que $\beta+\left(-\beta-\mu_{s}\right)=-\mu_{s} \in\langle-\alpha\rangle\left(\Phi_{-}(\Delta)\right)$. Por outro lado, se $\beta-\mu_{s} \in \Phi_{-}(\Delta)$, então $-\mu_{s} \in\langle-\alpha\rangle\left(\Phi_{-}(\Delta)\right)$, implicando que $\beta-\mu_{s} \in\langle-\alpha\rangle\left(\Phi_{-}(\Delta)\right)$. Neste caso, $-\mu_{s} \in\langle-\alpha\rangle\left(\Phi_{-}(\Delta)\right)$ é tal que $\beta-\mu_{s} \in\langle\alpha\rangle$.

Proposição 2.4.3. Sejam $W_{1}$ e $W_{2}$ g-módulos de Harish-Chandra de dimensão infinita, então $W_{1} \otimes W_{2}$ não é um $\mathfrak{g}$-módulo uniformemente limitado.

Demonstração. Sejam $\Phi_{j}^{i}=\Phi^{i}\left(W_{j}\right)$ e $\Phi_{j}^{f}=\Phi^{f}\left(W_{j}\right)$ os conjuntos das raízes injetivas e localmente finitas para o $\mathfrak{g}$-módulo $W_{j}$, onde $j=1$, 2. Seja $P_{1}=\Phi_{1}^{f} \cap-\Phi_{1}^{i}$ e $P_{2}=\Phi_{2}^{f} \cap-\Phi_{2}^{i}$ os conjuntos parabólicos que estudamos anteriormente. Seja $\Delta \subset \Phi$ uma base de raízes simples tal que $\Delta \subset P_{1}$ (existe pois $P_{1}$ é parabólico e pela Proposição 1.2.32). Se $\Phi_{1}^{i}=\Phi$, então $\Phi_{1}^{i} \cap \Phi_{2}^{i}=\Phi \cap \Phi_{2}^{i}=\Phi_{2}^{i}$. Como $W_{2}$ possui dimensão infinita e é simples, pelo Teorema 2.2.12, $\Phi_{2}^{i} \neq \emptyset$. Pelo Lema 2.4.1, $W_{1} \otimes W_{2}$ não é uniformemente limitado.

Podemos supor que $\Phi_{1}^{i} \neq \Phi$. Como $W_{1}$ possui dimensão infinita, é simples e $\Phi_{1}^{i} \neq \Phi$, teremos que $W_{1}$ satisfaz a terceira possibilidade do Teorema 2.2.12, portanto $P_{1} \backslash-P_{1} \neq \emptyset$. Temos que existe $\alpha \in \Delta$ tal que $\alpha \in P_{1} \backslash-P_{1}$. Como $P_{1} \backslash-P_{1} \subset \Phi_{1}^{f}$, temos que $\alpha$ é uma raiz simples que é um elemento de $\Phi_{1}^{f}$.

Considere $\langle-\alpha\rangle=\langle-\alpha\rangle\left(\Phi_{-}(\Delta)\right)$ o ideal de $\Phi_{-}(\Delta)$ gerado por $-\alpha$. Pelo Lema 2.2.7, $P_{1} \backslash-P_{1}$ e $P_{1} \cap-P_{1}$ são fechados. Além disso, se $\tau \in P_{1} \backslash-P_{1}$ e $\nu \in \Phi_{+}(\Delta) \subset P_{1}$ são tais que $\tau+\nu \in \Phi$, então $\tau+\nu \in P \backslash-P_{1}$. Assim, $\Phi_{1}^{f} \backslash-\Phi_{1}^{f}=P_{1} \backslash-P_{1}$ é um ideal de $\Phi_{+}(\Delta)$. Logo, o ideal de $\Phi_{+}(\Delta)$ gerado por $\alpha$ está contido em $\Phi_{1}^{f} \backslash-\Phi_{1}^{f}$. Como $\Phi_{1}^{i} \backslash-\Phi_{1}^{i}=-\left(\Phi_{1}^{f} \backslash-\Phi_{1}^{f}\right)$, temos que $\langle-\alpha\rangle \subset \Phi_{1}^{i} \backslash-\Phi_{1}^{i}$.

Pelo Lema 2.4.1, podemos supor $\Phi_{2}^{i} \cap\left( \pm \Phi_{1}^{i}\right)=\emptyset$. Como $W_{2}$ possui dimensão infinita e é simples, pelo Teorema 2.2.12, $\Phi_{2}^{i} \neq \emptyset$. Seja $\beta \in \Phi_{2}^{i}$. Como $\Phi_{2}^{i} \cap\left( \pm \Phi_{1}^{i}\right)=\emptyset$ e $\langle-\alpha\rangle \subset \Phi_{1}^{i} \backslash-\Phi_{1}^{i}$, temos que $\beta \notin \pm\langle-\alpha\rangle$. Pelo Lema 2.4.2, existe $\gamma \in\langle-\alpha\rangle$ tal que $\beta+\gamma \in\langle-\alpha\rangle$.

Sejam $W_{1, \lambda} \subset W_{1}$ e $W_{2, \mu} \subset W_{2}$ dois subespaços de peso de $W_{1}$ e $W_{2}$, respectivamente. Para todo inteiro não negativo $n$, o espaço de peso $\lambda+\mu+n(\gamma+\beta)$ em $W_{1} \otimes W_{2}$ contém o subespaço

$$
\sum_{l=0}^{n} W_{1, \lambda+l \gamma+(n-l)(\gamma+\beta)} \otimes W_{2, \mu+l \beta} .
$$

Como $\gamma, \gamma+\beta \in \Phi_{1}^{i}$ e $\beta \in \Phi_{2}^{i}$, temos que cada subespao da soma acima é não nulo e a dimensão de $\left(W_{1} \otimes W_{2}\right)_{\lambda+\mu+n(\gamma+\beta)}$ será maior ou igual à $n+1$. Como isto vale para todo $n \in \mathbb{Z}_{\geq 0}$, não existe um majorante para as dimensões do espaços de peso $W_{1} \otimes W_{2}$, então $W_{1} \otimes W_{2}$ não é uniformemente limitado.

Espaços de peso de um $\mathfrak{g} \otimes A$-módulo são exatamente os espaços de peso da subálgebra de Lie $\mathfrak{g} \cong \mathfrak{g} \otimes 1 \subset \mathfrak{g} \otimes A$. Sendo assim, um $\mathcal{G}$-módulo de peso é uniformemente limitado se e somente se é uniformemente limitado quando visto como g-módulo. Como vimos, módulos de avaliação são produtos tensoriais de $\mathfrak{g}$-módulos. Portanto, a Proposição 2.4.3 nos permite determinar todos os módulos de avaliação uniformemente limitados.

Teorema 2.4.4 ([BLL15, Theorem 3.14]). Seja $V(\underline{m}, \underline{W})$ um $\mathfrak{g} \otimes A$-módulo de avaliação de HarishChandra, onde $\underline{W}=\left(W_{1}, \ldots, W_{r}\right)$. Então $V(\underline{m}, \underline{W})$ é uniformemente limitado se, e somente se, $W_{1}, \ldots, W_{r}$ são uniformemente limitados e no máximo um dos $W_{i}$ possui dimensão infinita.

Demonstração. Se $V(\underline{m}, \underline{W})$ é uniformemente limitado, então pela Proposição 2.4.3 segue que não mais que um dos $W_{i}$ possui dimensão infinita. Os espaços de peso de $V(\underline{m}, \underline{W})$ são da forma

$$
\sum_{\lambda=\lambda_{1}+\cdots+\lambda_{r}} W_{1}^{\lambda_{1}} \otimes \cdots \otimes W_{r}^{\lambda_{r}}
$$

onde $\lambda \in \mathfrak{h}^{*}$. Como é uniformemente limitado, existe $B \in \mathbb{Z}_{\geq 0}$ que majora a dimensão dos espaços de peso. Sendo assim, $B$ também majora a dimensão dos espaços de peso $W_{i}^{\lambda_{i}}, \lambda_{i} \in \mathfrak{h}^{*}$. Logo, $W_{i}$ é uniformemente limitado, para todo $i=1, \ldots, r$. 
Por outro lado, se $W_{1}, \ldots, W_{r}$ são uniformemente limitados tais que no máximo um possui dimensão infinita. Se todos possuem dimensão finita, então $V(\underline{m}, \underline{W})$ possui dimensão finita e, assim, é uniformemente limitado. Por outro lado, suponha que $V(\underline{m}, \underline{W})$, então algum $W_{i}$ possui dimensão infinita. Suponha, sem perda de generalidade, que $W_{1}$ possui dimensão infinita. Para $i=2, \ldots, r$, seja $N_{i}$ a quantidade de pesos que $W_{i}$ possui e $n_{i}$ a dimensão de $W_{i}$. Seja $N>0$ um inteiro que majora a dimensão dos espaços de peso de $W_{1}$. Seja $\lambda \in \Pi(V(\underline{m}, \underline{W}))$ e $T(\lambda)$ o conjunto dos pesos de $W_{1}$ tal que existem $\lambda_{i} \in \Pi\left(W_{i}\right), i=2, \ldots, r$, tais que $\lambda=\lambda_{1}+\lambda_{2}+\cdots+\lambda_{r}$. Logo, $T(\lambda)$ é finito e a cardinalidade do conjunto $\{T(\lambda): \lambda \in \Pi(V)\}$ é menor ou igual que $N_{2} N_{3} \cdots N_{r}$. Sendo assim,

$$
V(\underline{m}, \underline{W})_{\lambda} \subset\left(\sum_{\lambda_{1} \in T(\lambda)} W_{\lambda_{1}}\right) \otimes W_{2} \cdots \otimes W_{r}
$$

possui dimensão menor que $N N_{2} N_{3} \cdots N_{r} n_{2} \cdots n_{r}$, implicando que $V(\underline{m}, \underline{W})$ é uniformemente limitado.

Agora que mostramos quando um módulo de Harish-Chandra de avaliação é uniformemente limitado, demonstraremos que todo $\mathcal{G}$-módulo de Harish-Chandra uniformemente limitado é um módulo de avaliação.

Lema 2.4.5. Se $\alpha \in \Phi^{+}$e $t \in A$, então, para todo $r \geq 1$,

$$
\left(x_{\alpha} \otimes t\right)\left(x_{-\alpha} \otimes 1\right)^{r}=\left(x_{-\alpha} \otimes 1\right)^{r}\left(x_{\alpha} \otimes t\right)+r\left(x_{-\alpha} \otimes 1\right)^{r-1}\left(h_{\alpha} \otimes t\right)-2\left(\begin{array}{l}
r \\
2
\end{array}\right)\left(x_{-\alpha} \otimes 1\right)^{r-2}\left(x_{-\alpha} \otimes t\right)
$$

$e$

$$
\left(x_{-\alpha} \otimes t\right)\left(x_{\alpha} \otimes 1\right)^{r}=\left(x_{\alpha} \otimes 1\right)^{r}\left(x_{-\alpha} \otimes t\right)-r\left(x_{\alpha} \otimes 1\right)^{r-1}\left(h_{\alpha} \otimes t\right)-2\left(\begin{array}{l}
r \\
2
\end{array}\right)\left(x_{\alpha} \otimes 1\right)^{r-2}\left(x_{\alpha} \otimes t\right)
$$

Demonstração. Demonstraremos apenas a primeira igualdade, visto que a prova das ambas igualdades são análogas. Denote $e=x_{\alpha}, f=x_{-\alpha}$ e $h=h_{\alpha}$. Provaremos por indução em $r \geq 1$. Se $r=1$, então

$$
(e \otimes t)(f \otimes 1)=(f \otimes 1)(e \otimes t)+(h \otimes t) .
$$

Além disso, quando $r=2$,

$$
\begin{aligned}
(e \otimes t)(f \otimes 1)^{2} & =(f \otimes 1)(e \otimes t)(f \otimes 1)+(h \otimes t)(f \otimes 1) \\
& =(f \otimes 1)^{2}(e \otimes t)+(f \otimes 1)(h \otimes t)+(h \otimes t)(f \otimes 1) \\
& =(f \otimes 1)(e \otimes t)+2(f \otimes 1)(h \otimes t)-2(f \otimes t)
\end{aligned}
$$

Suponha $r>2$ e que provamos para $r-1$. Note que

$$
\begin{aligned}
(h \otimes t)(f \otimes 1)^{r-1} & =(f \otimes 1)^{r-1}(h \otimes t)-2(r-1)(f \otimes 1)^{r-2}(f \otimes t) \\
& =(f \otimes 1)^{r-1}(h \otimes t)-2\left(\begin{array}{c}
r-1 \\
1
\end{array}\right)(f \otimes 1)^{r-2}(f \otimes t),
\end{aligned}
$$

portanto

$$
\begin{aligned}
(e \otimes t)(f \otimes 1)^{r}= & (f \otimes 1)(e \otimes t)(f \otimes 1)^{r-1}+2(h \otimes t)(f \otimes 1)^{r-1} \\
= & (f \otimes 1)^{r}(e \otimes t)+(r-1)(f \otimes 1)^{r-1}(h \otimes t) \\
& -2\left(\begin{array}{c}
r-1 \\
2
\end{array}\right)(f \otimes 1)^{r-3}(f \otimes t)+2(h \otimes t)(f \otimes 1)^{r-1} \\
= & (f \otimes 1)^{r}(e \otimes t)+r(f \otimes 1)^{r-1}(h \otimes t) \\
& \left.-2\left(\left(\begin{array}{c}
r-1 \\
2
\end{array}\right)+\left(\begin{array}{c}
r-1 \\
1
\end{array}\right)\right)(f \otimes 1)^{r-1}\right)(f \otimes t)
\end{aligned}
$$




$$
=(f \otimes 1)^{r}(e \otimes t)+r(f \otimes 1)^{r-1}(h \otimes t)-2\left(\begin{array}{l}
r \\
2
\end{array}\right)(f \otimes 1)^{r-2}(f \otimes t)
$$

Proposição 2.4.6 ( [BLL15, Proposition 4.4]). Seja $V$ um $\mathcal{G}$-módulo uniformemente limitado, então $I(V)$ é um ideal radical de $A$.

Demonstração. Seja $\rho: \mathcal{G} \rightarrow$ End $V$ a representação associada. Suponha que $I=I(V)$ não é um ideal radical de $A$ e seja $R=A / I$, onde $I$ é ideal de $A$ tal que $\mathfrak{g} \otimes I=\operatorname{ker}(\rho)$. Pela Proposição 2.1.15, $R$ é uma álgebra de dimensão finita (portanto artiniana) e a representação induzida $\hat{\rho}: \mathfrak{g} \otimes R \rightarrow$ End $V$ é injetora. Sendo assim, o nilradical de $R \sqrt{(0)}=\sqrt{I} / I$ é um ideal nilpotente. Seja $m>1$ tal que $(\sqrt{I} / I)^{m-1} \neq 0$ e $(\sqrt{I} / I)^{m}=0$. Seja $m^{\prime}$ o menor inteiro positivo maior ou igual $m / 2$ e defina $n=(\sqrt{I} / I)^{m^{\prime}}$, então $n \neq 0$ e $n^{2}=0$.

Note que $\mathfrak{h} \otimes n$ é uma álgebra de Lie abeliana de dimensão finita que comuta com $\mathfrak{h} \otimes 1$. Sendo assim, $(\mathfrak{h} \otimes n) V_{\lambda} \subset V_{\lambda}$ e, pelo Teorema de Lie (Teorema 1.2.8), existe um vetor $v \in V_{\lambda}$ não nulo tal que $(\mathfrak{h} \otimes n) v \subset \mathbb{k} v$, para todo $\lambda \in \mathfrak{h}^{*}$. Seja $\lambda$ um peso de $V$ e $0 \neq v \in V_{\lambda}$ tal que $(\mathfrak{h} \otimes n) v \subset \mathbb{k} v$. Para $x \in \mathfrak{h} \otimes n$, denotaremos por $\lambda(x)$ o autovalor da ação de $x$ em $v$. Seja $\Delta$ uma base de $\Phi$ e denote por $\mathfrak{g}_{ \pm}=\bigoplus_{\alpha \in \Phi_{ \pm}(\Delta)} \mathfrak{g}_{\alpha}$ e $\Phi_{ \pm}=\Phi_{+}(\Delta)$.

Afirmação 1: $\mathfrak{g}_{+} \otimes n$ e $\mathfrak{g}_{-} \otimes n$ agem nilpotentemente em $v$.

Como $\mathfrak{g}_{+}=\bigoplus_{\alpha \in \Phi_{+}} \mathfrak{g}^{\alpha}$, basta mostrar que $\mathfrak{g}^{\alpha} \otimes n$ age nilpotentemente em $v$. Seja $\alpha \in \Phi_{+}$e $a \in n$. Tome $x=h_{\alpha} \otimes a, y=x_{\alpha} \otimes 1$ e $z=2 x_{\alpha} \otimes a$. Seja $n \geq 0$ tal que $z^{n} v \neq 0$. Provaremos por indução em $n$ que o conjunto $\left\{z^{n-r} y^{r} v \mid r=0, \ldots, n\right\}$ é linearmente independente.

Para isto, note que $[x, z] \in \mathfrak{g} \otimes n=0$, portanto $[z, x]=[z, y]=0$. Além disso, os vendo como elementos de $U(\mathcal{G})$, temos que $x y=y z+z$ e, por indução em $r \geq 1$,

$$
x y^{r}=y^{r} x+r y^{r-1} z .
$$

Suponha que $n=1$ e sejam $c_{0}, c_{1} \in \mathbb{k}$ tais que $c_{0} z v+c_{1} y v=0$, então

$$
0=x\left(c_{0} z v+c_{1} y v\right)=\left(c_{0} z v+c_{1} y\right) x v+c_{1} z v=c_{1} z v .
$$

Como $z v \neq 0$, segue que $c_{1}=0$ e assim $c_{0}=0$. Suponha que $n>1$ e que $\left\{z^{n-r} y^{r} v \mid r=0, \ldots, n-1\right\}$ é linearmente independente. Sejam $c_{0}, \ldots, c_{n} \in \mathbb{k}$ tais que $C=\sum_{r=0}^{n} c_{r} z^{n-r} y^{r} v=0$, então

$$
\begin{aligned}
0=x C & =\sum_{r=0}^{n} c_{r} z^{n-r}\left(y^{r} x+r y^{r-1} z\right) v \\
& =\lambda(x) C+\sum_{r=1}^{n} c_{r} z^{n-r+1} y^{r-1} v \\
& =\sum_{r=1}^{n} r c_{r} z^{n-r+1} y^{r-1} v .
\end{aligned}
$$

Aplicando $x$ novamente, temos que $\sum_{r=2}^{n} r(r-1) c_{r} z^{n-r+2} y^{r-2} v=0$ e, portanto, aplicando $x$ em $C l \in\{0, \ldots, n\}$ vezes temos que

$$
\sum_{r=l}^{n} r(r-1) \ldots(r-(l-1)) c_{r} z^{n-r+l} y^{r-l} v=0 .
$$

Sendo assim, se $l=n$, então $n ! c_{n} z^{n} v=0$ e, como $z^{n} v \neq 0$, segue que $c_{n}=0$. Logo,

$$
\sum_{r=0}^{n-1} c_{r} z^{n-r} y^{r} v=0
$$


Portanto, se supormos $l=n-1$, temos que $(n-1) ! c_{n-1} z^{n} v=0$, implicando que $c_{n-1}=0$. Por indução, segue que $c_{r}=0$, para $r=0, \ldots, n$. Portanto,

$$
\left\{z^{n-r} y^{r} v \mid r=0, \ldots, n\right\}
$$

é linearmente independente.

Agora voltemos para a prova da Afirmação 1. Suponha que $z^{n} v \neq 0$ para todo $n \geq 0$. Como $z=2 x_{\alpha} \otimes a=\left[h_{\alpha} \otimes a, x_{\alpha} \otimes 1\right]$, segue que $z^{n-r} y^{r} v \in V_{\lambda+n \alpha}$, para cada $r=0, \ldots, n$. Como vimos $\left\{z^{n-r} y^{r} v: r=0, \ldots, n\right\} \subset V_{\lambda+n \alpha}$ é um conjunto linearmente independente com $n+1$ elementos. Portanto as dimensões dos espaços de peso de $V$ não são limitadas, contradizendo a hipótese de que $V$ é uniformemente limitado. Utilizando o mesmo argumento, porém supondo $x=h_{\alpha} \otimes 1$, $y=x_{-\alpha} \otimes 1$ e $z=-2 x_{-\alpha} \otimes 1$, temos uma prova que $\mathfrak{g}_{-} \otimes n$ age nilpotentemente.

Afirmação 2: Existe um vetor de peso $0 \neq w \in V$ tal que $(\mathfrak{g} \otimes n) w=0$.

Pela Afirmação 1, todo elemento da álgebra de dimensão finita $\mathfrak{g}_{+} \otimes n$ age nilpotentemente em $v$. Como $\mathfrak{g}_{+} \otimes n$ é abeliana, temos que $U\left(\mathfrak{g}_{+} \otimes n\right) v$ é um $\left(\mathfrak{g}_{+} \otimes n\right)$-módulo de dimensão finita tal que $\mathfrak{n}_{+} \otimes n$ age por transformações nilpotentes. Pelo Teorema de Engel, existe $u \in U\left(n_{+} \otimes n\right) v$ não nulo tal que $\left(\mathfrak{g}_{+} \otimes n\right) u=0$. Como $U\left(\mathfrak{g}_{+} \otimes n\right) v \subset V$ é um espaço de peso, podemos supor que $u$ é um vetor de peso.

Além disso, pela Afirmação 1 , temos que $\mathfrak{g}_{-} \otimes n$ age nilpotentemente em $u$. Utilizando um argumento parecido ao parágrafo anterior, temos que existe um vetor de peso $w \in U\left(\mathfrak{g}_{-} \otimes n\right) u$ tal que $\left(\mathfrak{g}_{-} \otimes n\right) w=0$. Como $\mathfrak{g}_{+} \otimes n$ comuta com $\mathfrak{g}_{-} \otimes n$ (pois $n^{2}=0$ ), temos que $\left(\mathfrak{g}_{+} \otimes n\right) w=0$. Logo,

$$
\left(\left(\mathfrak{g}_{-} \oplus \mathfrak{g}_{+}\right) \otimes n\right) w=0 .
$$

Como $w \in U\left(\left(\mathfrak{g}_{-} \oplus \mathfrak{g}_{+}\right) \otimes n\right) v$ e $\mathfrak{h} \otimes n$ comuta com $U\left(\left(\mathfrak{g}_{-} \oplus \mathfrak{g}_{+}\right) \otimes n\right)$, temos que $w$ é um autovetor para todo elemento de $\mathfrak{h} \otimes n$.

Para terminar de provar a Afirmação 2, mostraremos que $(\mathfrak{h} \otimes n) w=0$. Sejam $\alpha \in \Phi^{+}$e $t \in N$. Seja $c \in \mathbb{k}$ tal que $\left(h_{\alpha} \otimes t\right) w=c w$. Demonstraremos que $c=0$.

Como os espaços de pesos de $V$ possuem dimensão finita, temos que $\left\{\left(x_{\alpha} \otimes 1\right)^{r}\left(x_{-\alpha} \otimes 1\right)^{r} w \mid\right.$ $r \geq 0\}$ é linearmente dependente. Sejam $c_{0}, \ldots, c_{l} \in \mathbb{k}$ e $c_{l} \neq 0$ tais que

$$
\sum_{r=0}^{l} c_{r}\left(x_{\alpha} \otimes 1\right)^{r}\left(x_{-\alpha} \otimes 1\right)^{r} w=0,
$$

Sabemos que $\left(x_{\alpha} \otimes t\right) w=\left(x_{-\alpha} \otimes t\right) w=0$ e, usando o Lema 2.4.5, teremos que

$$
\begin{aligned}
0 & =\left(x_{\alpha} \otimes t\right)^{l}\left(x_{-\alpha} \otimes t\right)^{l} \sum_{r=0}^{l} c_{r}\left(x_{\alpha} \otimes 1\right)^{r}\left(x_{-\alpha} \otimes 1\right)^{r} w \\
& =\left(x_{\alpha} \otimes t\right)^{l}\left(x_{-\alpha} \otimes t\right)^{l-1} \sum_{r=1}^{l} r c_{r}\left(x_{-\alpha} \otimes 1\right)^{r-1}\left(x_{-\alpha} \otimes 1\right)^{r}\left(h_{\alpha} \otimes t\right) w \\
& =\left(x_{\alpha} \otimes t\right)^{l-1}\left(x_{-\alpha} \otimes t\right)^{l-1} \sum_{r=1}^{l}-r^{2} c_{r}\left(x_{-\alpha} \otimes 1\right)^{r-1}\left(x_{-\alpha} \otimes 1\right)^{r}\left(h_{\alpha} \otimes t\right)^{2} w \\
& =-c^{2}\left(x_{\alpha} \otimes t\right)^{l-1}\left(x_{-\alpha} \otimes t\right)^{l-1} \sum_{r=1}^{l} r^{2} c_{r}\left(x_{-\alpha} \otimes 1\right)^{r-1}\left(x_{-\alpha} \otimes 1\right)^{r-1} w \\
& =(-1)^{2} c^{4}\left(x_{\alpha} \otimes t\right)^{l-2}\left(x_{-\alpha} \otimes t\right)^{l-2} \sum_{r=2}^{l} r^{2}(r-1)^{2} c_{r}\left(x_{-\alpha} \otimes 1\right)^{r-2}\left(x_{-\alpha} \otimes 1\right)^{r-2} w \\
& =(-1)^{l} c^{2 l} l !^{2} c_{l} w .
\end{aligned}
$$

Como $c_{l} \neq 0$ e $w \neq 0$, segue que $c=0$. Logo, $(\mathfrak{h} \otimes n) w=0$, implicando que $(\mathfrak{g} \otimes n) w=0$. 
Afirmação 3: I é um ideal radical.

Seja $W=\{w \in V \mid(\mathfrak{g} \otimes n) w=0\}$. Note que

$$
(y \otimes b)(x \otimes a) w=(x \otimes a)(y \otimes b) w+([x, y] \otimes b a) w=0,
$$

para todo $(x \otimes a) \in \mathfrak{g} \otimes R$ e $y \otimes b \in \mathfrak{g} \otimes n$, pois $b a \in n$, já que $n$ é um ideal. Sendo assim, junto com a Afirmação $2, W$ é um $\mathfrak{g} \otimes R$-submódulo não nulo de $V$. Como $V$ é simples, segue que $V=W$. Portanto, $0 \neq \mathfrak{g} \otimes n \subset$ ker $\hat{\rho}$, contradizendo o fato que $\hat{\rho}: \mathfrak{g} \otimes R \rightarrow \operatorname{End} V$ é injetora. Logo, $I$ é um ideal radical.

Teorema 2.4.7 ( [BLL15, Theorem 4.5]). Seja V um $\mathcal{G}$-módulo de Harish-Chandra uniformemente limitado, então $V$ é isomorfo a um módulo de avaliação.

Demonstração. Sejam $\rho: \mathcal{G} \rightarrow \operatorname{End} V$ a representação associada e $I=I(V)$ o ideal de A tal que ker $\rho=\mathfrak{g} \otimes I$. Considere a representação $\hat{\rho}:(\mathfrak{g} \otimes A) /(\mathfrak{g} \otimes I) \rightarrow \operatorname{End} V$ injetora tal que $\rho=\hat{\rho} \circ \pi$, onde $\pi: \mathfrak{g} \otimes A \rightarrow \mathfrak{g} \otimes A /$ ker $\rho$ é a projeção canônica. Pela Proposição 2.4.6, temos que $I$ é um ideal radical de $A$, logo existem ideais maximais distintos $m_{1}, \ldots, m_{r}$ de $A$ tais que $I=m_{1} \cap \cdots \cap m_{r}$. Pelo Teorema Chinês do Resto (Teorema 1.1.13),

$$
\mathfrak{g} \otimes A / \operatorname{ker} \rho \cong \mathfrak{g} \otimes(A / I) \cong \mathfrak{g} \otimes\left(A / \bigcap_{i=1}^{r} m_{i}\right) \cong \mathfrak{g} \otimes\left(A / m_{1} \oplus \cdots \oplus A / m_{r}\right)
$$

Como $A$ é finitamente gerada e $\mathbb{k}$ é algebricamente fechado, segue que $A / m_{i} \cong \mathbb{k}$ e este isomorfismo é dado por $a+m_{i} \mapsto a\left(m_{i}\right)$, para cada $i=1, \ldots, r$. Logo,

$$
\mathfrak{g} \otimes A / \operatorname{ker} \rho \cong \mathfrak{g}^{\oplus r} .
$$

Seja $\psi: \mathfrak{g} \otimes A / \operatorname{ker} \rho \rightarrow \mathfrak{g}^{\oplus r}$ o isomorfismo acima e $e v \underline{m}: \mathfrak{g} \otimes A \rightarrow \mathfrak{g}^{\oplus r}$, onde $\underline{m}=\left(m_{1}, \ldots, m_{r}\right)$. Note que $\mathrm{evm}=\psi \circ \pi$. Portanto, $\rho=\hat{\rho} \circ \psi^{-1} \circ \psi \circ \pi=\left(\hat{\rho} \circ \psi^{-1}\right) \circ \mathrm{evm}$. Pelo Teorema 2.3.6, segue que $V$ isomorfo à um módulo de avaliação.

\subsection{Módulos de Avaliação de Módulos de Indução Parabólica}

Seja $P \subset \Phi$ um subconjunto parabólico qualquer de $\Phi$ e considere a subálgebra de $\mathfrak{g}$

$$
\mathfrak{l}=\mathfrak{h} \oplus \sum_{\alpha \in P \cap-P} \mathfrak{g}_{\alpha}
$$

Defina $\mathcal{L}=\mathfrak{l} \otimes A$. Como $\mathfrak{l}$ é uma álgebra de Lie redutível, existe uma álgebra de Lie semissimples $\mathfrak{s}$ e um álgebra de Lie abeliana $\mathfrak{z}$ tal que a soma direta de álgebras de Lie $\mathfrak{l}=\mathfrak{s} \oplus \mathfrak{s}$. Pela Proposição 2.1.17, os $\mathcal{L}$-módulos uniformemente limitados simples $W$ são isomorfos ao produto tensorial

$$
W \cong U \otimes \mathbb{k} v_{\Lambda},
$$

onde $U$ é um $\mathfrak{s} \otimes A$-módulo de Harish-Chandra uniformemente limitado e $\Lambda$ é uma transformação linear tal que $(z \otimes a) v_{\Lambda}=\Lambda(z \otimes a)$ para todo $z \otimes a \in \mathfrak{z} \otimes A$.

Pela Proposição 2.1 .17 e Teorema 2.4.7, existem $\underline{m}=\left(m_{1}, \ldots, m_{q}\right)$ uma $q$-upla de ideais maximais distintos de $A$ e $\underline{W}=\left(W_{1}, \ldots, W_{q}\right)$ uma $q$-upla de l-módulos uniformemente limitados simples tais que

$$
W \cong W_{1}\left(m_{1}\right) \otimes \cdots \otimes W_{q}\left(m_{q}\right) \otimes \mathbb{k} v_{\Lambda}=V(\underline{m}, \underline{W}) \otimes \mathbb{k} v_{\Lambda} .
$$

Note que $\mathbb{k}_{\Lambda}$ pode não ser um módulo de avaliação sobre $\mathfrak{z} \otimes A$ e, portanto, poderia não se misturar com os outros elementos do produto tensorial. Se $\mathbb{k}_{\Lambda}$ é um módulo de avaliação, então existem ideais maximais $n_{1}, \ldots, n_{s}$ distintos de $A$ e funcionais lineares $\lambda_{1}, \ldots, \lambda_{s}$ tais que $\Lambda(h \otimes a)=$ 
$\sum_{i=1}^{s} a\left(n_{i}\right) \lambda_{i}(h)$. Se $m_{i}=n_{j}$, então, pela Proposição 1.3.22, $W_{i} \otimes \mathbb{k} v_{\lambda_{j}}$ é um $\mathfrak{s} \oplus \mathfrak{z}$-módulo simples e, portanto, $\left(W_{i} \otimes \mathbb{k} v_{\lambda_{j}}\right)\left(m_{i}\right)$ é um $\mathcal{L}$-módulo simples. Ou seja, se $\mathbb{k}_{\Lambda}$ é um $\mathfrak{z} \otimes A$-módulo de avaliação, então existem ideais maximais distintos $m_{1}, \ldots, m_{r}$ de $A$ e $W_{1}, \ldots, W_{r}$ l-módulos simples tais que

$$
W \cong W_{1}\left(m_{1}\right) \otimes \cdots \otimes W_{r}\left(m_{r}\right) .
$$

Suponha que $\mathbb{k} v_{\Lambda}$ seja um $\mathfrak{z} \otimes A$-módulo de avaliação. Como feito na Proposição 2.2.9, considere as subálgebras de $\mathfrak{g}$

$$
\begin{gathered}
\mathfrak{n}_{ \pm}=\sum_{ \pm \alpha \in P \backslash-P} \mathfrak{g}_{\alpha}, \\
\mathfrak{p}=\mathfrak{h} \oplus \sum_{\alpha \in P} \mathfrak{g}_{\alpha}=\mathfrak{l} \oplus \mathfrak{n}_{+}
\end{gathered}
$$

e as subálgebras de $\mathcal{G}$ dadas por $\mathcal{N}_{ \pm}=\mathfrak{n}_{ \pm} \otimes A$ e $\mathcal{P}=\mathfrak{p} \otimes A$. Seja $\Delta$ o homomorfismo de álgebras associativas com unidade

$$
\Delta: U(\mathcal{G}) \rightarrow U(\mathcal{G})^{\otimes r}
$$

definido por

$$
\Delta(x)=\sum_{i=1}^{r} x_{i}
$$

onde $x_{i}$ é igual ao produto tensorial $1 \otimes \cdots \otimes x \otimes \cdots \otimes 1$ que possui $x$ na $i$-ésima posição e 1 nas demais. Pela Proposição 2.2.10, o módulo de Verma generalizado $M_{\mathcal{P}}(W)$ é livre quando visto como um $U\left(\mathcal{N}_{-}\right)$-módulo. Note que $U(\mathcal{G})^{\otimes r}$ é um $U(\mathcal{G})$-módulo com a ação dada por

$$
x\left(u_{1} \otimes \cdots \otimes \ldots u_{r}\right)=\Delta(x)\left(u_{1} \otimes \cdots \otimes u_{r}\right)=\sum_{i=1}^{r} u_{1} \otimes \cdots \otimes x u_{i} \otimes \cdots \otimes u_{r},
$$

onde $x \in U(\mathcal{G})$ e $u_{1} \otimes \cdots \otimes u_{r} \in U(\mathcal{G})^{\otimes r}$.

Lema 2.5.1. A aplicação

$$
\begin{aligned}
\delta: M_{\mathcal{P}}(W) & \rightarrow \bigotimes_{i=1}^{r} M_{\mathcal{P}}\left(W_{i}\left(m_{i}\right)\right) \\
u \otimes_{U(\mathcal{P})}\left(w_{1} \otimes \cdots \otimes w_{r}\right) & \mapsto \Delta(u)\left(\hat{w}_{1} \otimes \cdots \otimes \hat{w}_{r}\right)
\end{aligned}
$$

é um homomorfismo de $\mathcal{G}$-módulos, onde $u \in \mathcal{G}, \hat{w}_{i}=1 \otimes_{U(\mathcal{P})} w_{i} \in M_{\mathcal{P}}\left(W_{i}\left(m_{i}\right)\right)$ e $\left(u_{1} \otimes \cdots \otimes\right.$ $\left.u_{r}\right)\left(\hat{w}_{1} \otimes \cdots \otimes \hat{w}_{r}\right)$ é definido como $u_{1} \hat{w}_{1} \otimes \cdots \otimes u_{r} \hat{w}_{r}$ para cada $u_{i} \in U(\mathcal{G})$ e $w_{i} \in W_{i}\left(m_{i}\right)$.

Demonstração. Precisamos mostrar que $\delta$ está bem definida. Seja $w_{1} \otimes \cdots \otimes w_{r} \in \bigotimes_{i=1}^{r} W_{i}\left(m_{i}\right)$ não nulo, então $W=U(\mathcal{P}) w_{1} \otimes \cdots \otimes w_{r}$, pois $W$ é um $U(\mathcal{L})$-módulo simples. Portanto, para mostrar que $\delta$ está bem definida, basta mostrar que se $w_{1} \otimes \cdots \otimes w_{r}=x\left(w_{1} \otimes \cdots \otimes w_{r}\right)$, para $x \in U(\mathcal{P})$, então

$$
\delta\left(u \otimes_{U(\mathcal{P})}\left(w_{1} \otimes \cdots \otimes w_{r}\right)\right)=\delta\left(u \otimes_{U(\mathcal{P})} x\left(w_{1} \otimes \cdots \otimes w_{r}\right)\right) .
$$

Note que $x\left(w_{1} \otimes \cdots \otimes w_{r}\right)=\Delta(x)\left(w_{1} \otimes \cdots \otimes w_{r}\right)$ e $x\left(\hat{w}_{1} \otimes \cdots \otimes \hat{w}_{r}\right)=\Delta(x)\left(\hat{w}_{1} \otimes \cdots \otimes \hat{w}_{r}\right)$, portanto

$$
\begin{aligned}
\delta\left(u \otimes_{U(\mathcal{P})} x\left(w_{1} \otimes \cdots \otimes w_{r}\right)\right) & =\delta\left((u x) \otimes_{U(\mathcal{P})}\left(w_{1} \otimes \cdots \otimes w_{r}\right)\right) \\
& =\Delta(u x)\left(\hat{w}_{1} \otimes \cdots \otimes \hat{w}_{r}\right) \\
& =\Delta(u) \Delta(x)\left(\hat{w}_{1} \otimes \cdots \otimes \hat{w}_{r}\right) \\
& =\Delta(u) \delta\left(x \otimes_{U(\mathcal{P})}\left(w_{1} \otimes \cdots \otimes w_{r}\right)\right) \\
& =\Delta(u) \delta\left(1 \otimes_{U(\mathcal{P})} x\left(w_{1} \otimes \cdots \otimes w_{r}\right)\right) \\
& =\Delta(u) \Delta(1)\left(\hat{w}_{1} \otimes \cdots \otimes \hat{w}_{r}\right) \\
& =\Delta(u)\left(\hat{w}_{1} \otimes \cdots \otimes \hat{w}_{r}\right)
\end{aligned}
$$




$$
=\delta\left(u \otimes_{U(\mathcal{P})}\left(w_{1} \otimes \cdots \otimes w_{r}\right)\right)
$$

Sendo assim, $\delta$ está bem definido e, pela linearidade de $\Delta$ e pelas propriedades do produto tensorial, $\delta$ é uma aplicação linear. Por outro lado, para todo $x \in U(\mathcal{G})$ e $u \otimes_{U(\mathcal{P})}\left(w_{1} \otimes \cdots \otimes w_{r}\right) \in M_{\mathcal{P}}(W)$,

$$
\begin{aligned}
\delta\left(x\left(u \otimes_{U(\mathcal{P})}\left(w_{1} \otimes \cdots \otimes w_{r}\right)\right)\right) & =\delta\left(x u \otimes_{U(\mathcal{P})}\left(w_{1} \otimes \cdots \otimes w_{r}\right)\right) \\
& =\Delta(x u)\left(\hat{w}_{1} \otimes \cdots \otimes \hat{w}_{r}\right) \\
& =\Delta(x) \Delta(u)\left(\hat{w}_{1} \otimes \cdots \otimes \hat{w}_{r}\right) \\
& =x \delta\left(u \otimes_{U(\mathcal{P})}\left(w_{1} \otimes \cdots \otimes w_{r}\right)\right)
\end{aligned}
$$

Portanto $\delta$ é um homomorfismo de $U(\mathcal{G})$-módulos.

Visto que $W_{i}$ é um l-módulo simples e $\mathfrak{p}=\mathfrak{n}_{+} \oplus \mathfrak{l}$ é uma subálgebra parabólica de $\mathfrak{g}$, podemos construir o módulo de Verma generalizado

$$
M_{\mathfrak{p}}\left(W_{i}\right)=U(\mathfrak{g}) \otimes_{U(\mathfrak{p})} W_{i}
$$

Com este $\mathfrak{g}$-módulo, podemos construir o $\mathcal{G}$-módulo de avaliação $M_{\mathfrak{p}}\left(W_{i}\right)\left(m_{i}\right)$ utilizando o ideal maximal $m_{i}$ de $A$, onde $(x \otimes a) w=a\left(m_{i}\right) x w$, onde $x \otimes a \in \mathcal{G}$ e $w \in M_{\mathfrak{p}}\left(W_{i}\right)$. Para cada $u \in U(\mathcal{G})$, definimos $u\left(m_{i}\right)$ como sendo a imagem pelo homomorfismo de álgebras associativas com unidade $U(\mathcal{G}) \rightarrow U(\mathfrak{g})$ definido pela extensão da aplicação de avaliação $x \otimes a \mapsto a\left(m_{i}\right) x$, para cada $x \otimes a \in \mathcal{G}$.

Lema 2.5.2. A aplicação

$$
\begin{aligned}
\mathrm{ev}_{i}: M_{\mathcal{P}}\left(W_{i}\left(m_{i}\right)\right) & \rightarrow M_{\mathfrak{p}}\left(W_{i}\right)\left(m_{i}\right) \\
u \otimes_{U(\mathcal{P})} w_{i} & \mapsto u\left(m_{i}\right) \otimes_{U(\mathfrak{p})} w_{i}
\end{aligned}
$$

é um homomorfismo de $\mathcal{G}$-módulos.

Demonstração. Como $u \mapsto u\left(m_{i}\right)$ é uma transformação linear, temos que $e v_{i}$ também está bem definida e é linear. Sabemos que $U(\mathfrak{g})$ é um módulo sobre $U(\mathcal{G})$ com a ação dada por $x u=x\left(m_{i}\right) y$, onde $x \in U(\mathcal{G})$ e $u \in U(\mathfrak{g})$, portanto

$$
\begin{aligned}
\mathrm{ev}_{i}\left(x\left(u \otimes_{U(\mathcal{P})} w_{i}\right)\right) & =\mathrm{ev}_{i}\left((x u) \otimes_{U(\mathcal{P})} w_{i}\right) \\
& =(x u)\left(m_{i}\right) \otimes_{U(\mathfrak{p})} w_{i} \\
& =\left(x\left(m_{i}\right) u\left(m_{i}\right)\right) \otimes_{U(\mathfrak{p})} w_{i} \\
& =x\left(u\left(m_{i}\right) \otimes_{U(\mathfrak{p})} w_{i}\right) \\
& =x \operatorname{ev}_{i}\left(u \otimes_{U(\mathfrak{p})} w_{i}\right),
\end{aligned}
$$

para todo $x, u \in U(\mathcal{G})$ e $w_{i} \in W$. Logo, ev $i$ é homomorfismo de $\mathcal{G}$-módulos.

Lema 2.5.3. A aplicação

$$
\begin{aligned}
\mathrm{ev}: & \bigotimes_{i=1}^{r} M_{\mathcal{P}}\left(W_{i}\left(m_{i}\right)\right) \rightarrow \bigotimes_{i=1}^{r} M_{\mathfrak{p}}\left(W_{i}\right)\left(m_{i}\right) \\
v_{1} \otimes \cdots \otimes v_{r} & \mapsto \operatorname{ev}_{1}\left(v_{1}\right) \otimes \cdots \otimes \mathrm{ev}_{r}\left(v_{r}\right)
\end{aligned}
$$

é um homomorfismo de $\mathcal{G}$-módulos.

Demonstração. Como $\mathrm{ev}_{1}, \ldots, \mathrm{ev}_{r}$ são transformações lineares e a aplicação

$$
\bigoplus_{i=1}^{r} M_{\mathcal{P}}\left(W_{i}\left(m_{i}\right)\right) \rightarrow \bigoplus_{i=1}^{r} M_{\mathfrak{p}}\left(W_{i}\right)\left(m_{i}\right)
$$




$$
\left(v_{1}, \ldots, v_{r}\right) \mapsto\left(\operatorname{ev}_{1}\left(v_{1}\right), \ldots, \mathrm{ev}_{r}\left(v_{r}\right)\right)
$$

é r-linear, então ev é uma transformação linear bem definida. Além disso,

$$
\begin{aligned}
\mathrm{ev}\left(x\left(v_{1} \otimes \cdots \otimes v_{r}\right)\right) & =\sum_{i=1}^{r} \operatorname{ev}\left(v_{1} \otimes \cdots \otimes x v_{i} \otimes \cdots \otimes v_{r}\right) \\
& =\sum_{i=1}^{r} \operatorname{ev}_{1}\left(v_{1}\right) \otimes \cdots \otimes \mathrm{ev}_{r}\left(x v_{i}\right) \otimes \cdots \otimes \mathrm{ev}_{r}\left(v_{r}\right) \\
& =\sum_{i=1}^{r} \operatorname{ev}_{1}\left(v_{1}\right) \otimes \cdots \otimes x\left(m_{i}\right) \mathrm{ev}_{r}\left(v_{i}\right) \otimes \cdots \otimes \mathrm{ev}_{r}\left(v_{r}\right) \\
& =x\left(\operatorname{ev}_{1}\left(v_{1}\right) \otimes \cdots \otimes \mathrm{ev}_{r}\left(v_{r}\right)\right) \\
& =x \operatorname{ev}\left(v_{1} \otimes \cdots \otimes v_{r}\right),
\end{aligned}
$$

para todo $x \in U(\mathcal{G})$ e $v_{1} \otimes \cdots \otimes v_{r} \in \bigotimes_{i=1}^{r} M_{\mathcal{P}}\left(W_{i}\left(m_{i}\right)\right)$.

Para cada $i=1, \ldots, r$, considere a aplicação canônica $\pi_{i}: M_{\mathfrak{p}}\left(W_{i}\right)\left(m_{i}\right) \rightarrow L_{\mathfrak{p}}\left(W_{i}\right)\left(m_{i}\right)$. É possível mostrar que $\pi_{1}, \ldots, \pi_{r}$ são homomorfismos de $\mathcal{G}$-módulos, pois são homomorfismos de $\mathfrak{g}$ módulos e $x v_{i}=x\left(m_{i}\right) v_{i}$ para todo $x \in U(\mathcal{G})$, onde $x\left(m_{i}\right) \in \mathfrak{g}$. Utilizando uma prova semelhante a dada no Lema anterior, é possível mostrar o seguinte lema.

Lema 2.5.4. A aplicação

$$
\begin{aligned}
& \pi: \bigotimes_{i=1}^{r} M_{\mathfrak{p}}\left(W_{i}\right)\left(m_{i}\right) \rightarrow \bigotimes_{i=1}^{r} L_{\mathfrak{p}}\left(W_{i}\right)\left(m_{i}\right) \\
& m_{1} \otimes \cdots \otimes m_{r} \mapsto \pi_{1}\left(m_{1}\right) \otimes \cdots \otimes \pi_{r}\left(m_{r}\right)
\end{aligned}
$$

é um homomorfismo de $\mathcal{G}$-módulos.

Com estes lemas, estamos aptos pra provar o primeiro resultado desta seção:

Proposição 2.5.5 ( [Lau18, Proposition 4.1]). Seja V um $\mathcal{G}$-módulo de Harish-Chandra, então existe um conjunto parabólico $P \subset \Phi$ e um $\mathcal{L}=\mathfrak{l} \otimes A$-módulo simples uniformemente limitado $W$ tal que $V \cong L_{\mathcal{P}}(W)$, onde $\mathfrak{l}=\mathfrak{h} \oplus \sum_{\alpha \in P \cap-P} \mathfrak{g}_{\alpha}$. Seja $\mathfrak{z}$ o centro de $\mathfrak{l}$. Se $W$ é um $\mathfrak{z} \otimes A$ módulo de avaliação, então existe uma família finita $W_{1}, \ldots, W_{r}$ de módulos de Harish-Chandra uniformemente limitados sobre $\mathfrak{l}=\mathfrak{h} \oplus \sum_{\alpha \in P \cap-P} \mathfrak{g}_{\alpha}$ e ideais maximais $m_{1}, \ldots, m_{r}$ de $A$ distintos tais que $W_{1} \otimes \cdots \otimes W_{r}$ é um $\mathfrak{l}$-módulo uniformemente limitado e

$$
V \cong \bigotimes_{i=1}^{r} L_{\mathfrak{p}}\left(W_{i}\right)\left(m_{i}\right)
$$

Demonstração. Pela Proposição 2.2.10 e pelo Teorema 2.4.7, já temos que $V \cong L_{\mathcal{P}}(W)$, onde $W \cong W_{1}\left(m_{1}\right) \otimes \cdots \otimes W_{r}\left(m_{r}\right) \otimes \mathbb{k} v_{\Lambda}$ é um $\mathcal{L}$-módulo simples uniformemente limitado. Como a ação de $\mathfrak{z} \otimes A$ em $W$ é dada por $\Lambda$, temos que $(\mathfrak{z} \otimes A) W=0$ se, e somente se, $\Lambda=0$. Basta mostrar que $V \cong \bigotimes_{i=1}^{r} L_{\mathfrak{p}}\left(W_{i}\right)\left(m_{i}\right)$. Considere os homomorfismo de $\mathcal{G}$-módulos não nulos $\delta$, ev e $\pi$ construídos nos lemas anteriores, então

$$
\pi \circ \mathrm{ev} \circ \delta: M_{\mathcal{P}}(W) \rightarrow \bigotimes_{i=1}^{r} L_{\mathfrak{p}}\left(W_{i}\right)\left(m_{i}\right)
$$

é um homomorfismo de $\mathcal{G}$-módulos não nulo. Pela Proposição 2.3.5, o $\mathcal{G}$-módulo de avaliação $\bigotimes_{i=1}^{r} L_{\mathfrak{p}}\left(W_{i}\right)\left(m_{i}\right)$ é simples, portanto $\pi \circ$ ev $\circ \delta$ é sobrejetor. É possível mostrar que $\operatorname{ker}(\pi \circ \mathrm{ev} \circ \delta) \subset$ 
$U\left(\mathcal{N}_{-}\right) \mathcal{N}_{-} W$ e, como $\bigotimes_{i=1}^{r} L_{\mathfrak{p}}\left(W_{i}\right)\left(m_{i}\right)$ é simples, $\operatorname{ker}(\pi \circ$ evo $\delta)$ é maximal. Pela Proposição 2.2.10,

$$
V \cong L_{\mathcal{P}}(W) \cong \bigotimes_{i=1}^{r} L_{\mathfrak{p}}\left(W_{i}\right)\left(m_{i}\right)
$$

Por fim, iremos mostrar quando o módulo de avaliação de g-módulos de indução parabólica são $\mathcal{G}$-módulos de Harish-Chandra.

Proposição 2.5.6 ( [Lau18, Proposition 4.2]). Seja $P \subset \Phi$ um subconjunto parabólico e $\mathfrak{l}=$ $\mathfrak{h} \oplus \sum_{\alpha \in P \cap-P} \mathfrak{g}_{\alpha}$. Sejam $W_{1}, \ldots, W_{r} \mathfrak{l}$-módulos de Harish-Chandra tais que $W_{1} \otimes \cdots \otimes W_{r}$ é um $\mathfrak{l}$-módulo quasi-finito, então $\bigotimes_{i=1}^{r} L_{\mathfrak{p}}\left(W_{i}\right)\left(m_{i}\right)$ é um $\mathcal{G}$-módulo de Harish-Chandra para toda família de ideais maximais distintos $m_{1}, \ldots, m_{r}$ de $A$.

Demonstração. Sejam $m_{1}, \ldots, m_{r}$ ideais distintos de $A$ e $\mathfrak{p}=\mathfrak{h} \oplus \sum_{\alpha \in P} \mathfrak{g}_{\alpha}$. Denote por $W=$ $W_{1} \otimes \cdots \otimes W_{r}, D \subset \mathfrak{h}^{*}$ o conjunto de pesos de $W, \mathcal{Q}_{0} \subset \mathcal{Q}$ o reticulado de raízes gerado por $P \cap-P$ e $C=\operatorname{span}_{\mathbb{Z}_{>0}}((-P) \backslash P)$ o conjunto de pesos de $U\left(\mathfrak{n}_{-}\right)$visto como $\mathfrak{g}$-módulo. Note que $D \subset \lambda+\mathcal{Q}_{0}$ se $\lambda \in D$. Pela Proposição 2.3.5, o $\mathcal{G}$-módulo de avaliação $V=\bigotimes_{i=1}^{r} L_{\mathfrak{p}}\left(W_{i}\right)\left(m_{i}\right)$ é simples.

Falta mostrar que

$$
\bigotimes_{i=1}^{r} L_{\mathfrak{p}}\left(W_{i}\right)\left(m_{i}\right)
$$

é um $\mathcal{G}$-módulo quasi-finito. Para isto, basta mostrar que o $\mathfrak{g} \cong \mathfrak{g} \otimes 1$-módulo

$$
L_{\mathfrak{p}}\left(W_{1}\right) \otimes \cdots \otimes L_{\mathfrak{p}}\left(W_{r}\right)
$$

é um módulo quasi-finito. Fixe $\lambda \in D$. Os pesos de

$$
\bigotimes_{i=1}^{r} M_{\mathfrak{p}}\left(W_{i}\right)
$$

são da forma $\lambda+c+\mu$, onde $c \in C$ e $\mu \in \mathcal{Q}_{0}$. Além disso, cada $M_{\mathfrak{p}}\left(W_{1}\right), \ldots, M_{\mathfrak{p}}\left(W_{r}\right)$ é um $\mathfrak{g}$-módulo quasi-finito. Note que $\mathcal{Q}_{0} \cap C=0$. Seja $\gamma \in \mathfrak{h}^{*}$ tal que existe $c \in C$ e $\mu \in \mathcal{Q}_{0}$ tal que $\gamma=\lambda+c+\mu$. Seja $\left\{\alpha_{1}, \ldots, \alpha_{q}\right\} \subset \Phi$ uma base de raízes simples que está contida em $P$ tal que $\left\{\alpha_{1}, \ldots, \alpha_{l}\right\}$ é uma base do sistema de raízes $P \cap-P$, então

$$
\mu=\sum_{i=1}^{l} k_{i} \alpha_{i} \quad \text { e } \quad c=\sum_{i=1}^{r} k_{i}^{\prime} \alpha_{i}
$$

onde $k_{1}, \ldots, k_{l} \in \mathbb{Z}, k_{1}^{\prime}, \ldots, k_{q}^{\prime} \in \mathbb{Z}_{\leq 0}$ e $\sum_{i=1}^{r} k_{i}^{\prime} \alpha_{i} \in \mathcal{Q}_{0}$ é não nulo somente se $\sum_{i=l+1}^{r} k_{i}^{\prime} \alpha_{i} \neq 0$. Se $c=0$, então $\mu$ é o único elemento de $\mathcal{Q}_{0}$ tal que $\gamma=\lambda+c+\mu$. Se $c \neq 0$, então existe $l+1 \leq i \leq q$ tal que $k_{i}^{\prime} \neq 0$. Assim, se $\left(c^{\prime}, \mu^{\prime}\right) \in C \times \mathcal{Q}_{0}$ é um par tal que $\gamma=\lambda+c^{\prime}+\mu^{\prime}$, então existe $\mu^{\prime \prime} \in \mathcal{Q}_{0}$ tal que

$$
c^{\prime}=\mu^{\prime \prime}+\sum_{i=l+1}^{q} k_{i}^{\prime} \alpha_{i} \quad \text { e } \quad \mu^{\prime}+\mu^{\prime \prime}=\sum_{i=1}^{l}\left(k_{i}+k_{i}^{\prime}\right) \alpha_{i} .
$$

Portanto, para todo peso $\gamma$ de $\bigotimes_{i=1}^{r} M_{\mathfrak{p}}\left(W_{i}\right)$ existe uma quantidade finita de pares $(c, \mu) \in C \times \mathcal{Q}_{0}$ com $c+\mu+\lambda=\gamma$. Similarmente, para cada $\mu \in D$, existe uma quantidade finita de escolhas $\mu_{1}, \ldots, \mu_{r}$ entre os pesos de $W_{1}, \ldots, W_{r}$, respectivamente, tais que $\mu=\mu_{1}+\cdots+\mu_{r}$, pois $W_{1} \otimes$ $\cdots \otimes W_{r}$ é um l-módulo quasi-finito.

Logo, se $\lambda+c+\mu$ é um peso de $\bigotimes_{i=1}^{r} M_{\mathfrak{p}}\left(W_{i}\right)$, então o espaço de peso correspondente será uma soma finita de espaços de dimensão finita e, portanto, possui dimensão finita. Concluímos que $\bigotimes_{i=1}^{r} M_{\mathfrak{p}}\left(W_{i}\right)$ é um $\mathfrak{g}$-módulo quasi-finito, implicando que $L_{\mathfrak{p}}\left(W_{1}\right) \otimes \cdots \otimes L_{\mathfrak{p}}\left(W_{r}\right)$ é um $\mathfrak{g}$-módulo quasi-finito também. 


\subsection{Módulos de Peso Máximo}

Seja $\Delta$ uma base do sistema de raízes $\Phi$ de $\mathfrak{g}$. Lembre que $\mathfrak{g}$ admite uma decomposição triangular

$$
\mathfrak{g}=\mathfrak{g}_{-} \oplus \mathfrak{h} \oplus \mathfrak{g}_{+}
$$

Consideraremos as subálgebras de $\mathcal{G}$ dadas por $\mathcal{G}_{-}=\mathfrak{g}_{-} \otimes A, \mathcal{H}=\mathfrak{h} \oplus A$ e $\mathcal{G}_{+}=\mathfrak{g}_{+} \otimes A$, então

$$
\mathcal{G}=\mathcal{G}_{-} \oplus \mathcal{H} \oplus \mathcal{G}_{+}
$$

Denote por $\mathcal{Q}=\mathbb{Z} \Phi$ o reticulado de raízes gerado por $\Phi$ em $\mathfrak{h}^{*}$ e

$$
\mathcal{Q}_{ \pm}=\left\{ \pm \sum_{\alpha \in \Phi_{+}} c_{\alpha} \alpha \mid c_{\alpha} \in \mathbb{Z}, c_{\alpha} \geq 0\right\} \text {. }
$$

Lembre que $U\left(\mathcal{G}_{ \pm}\right)$é um hy-módulos de peso e seus pesos são subconjuntos de $\mathcal{Q}_{ \pm}$.

Definição 2.6.1. Sejam $\Lambda \in \mathcal{H}^{*}$. Dizemos que um $\mathcal{G}$-módulo $V$ é um módulo de peso máximo se existe um vetor $v \in V$ tal que

1. $U(\mathcal{G}) v=V$,

2. $\mathcal{G}_{+} v=0$,

3. existe um funcional linear $\Lambda \in \mathcal{H}^{*}$ tal que $h v=\Lambda(h) v$, para todo $h \in \mathcal{H}$.

Neste caso, dizemos que $\Lambda$ é um peso máximo de $V, v$ é um vetor de peso máximo.

O objetivo desta definição é criar algo semelhante ao definido para álgebras de Lie semissimples na Seção 1.4.

Observação 2.6.2. Seja $V$ é um $\mathcal{G}$-módulo de peso máximo $\Lambda \in \mathcal{H}$ e vetor de peso máximo $v$, então

$$
\begin{aligned}
V=U(\mathcal{G}) v & =U\left(\mathcal{G}_{-}\right) U(\mathcal{H}) U\left(\mathcal{G}_{+}\right) v \\
& =U\left(\mathcal{G}_{-}\right) U(\mathcal{H}) v \\
& =U\left(\mathcal{G}_{-}\right) v
\end{aligned}
$$

Além disso, se $\mu \in \mathcal{Q}_{+}, h \in \mathfrak{h}, a \in A$ e $u \in U\left(\mathcal{G}_{-}\right)_{-\mu}$, então

$$
\begin{aligned}
(h \otimes 1) u v & =u(h \otimes 1) v+\operatorname{ad}_{h \otimes 1}(u) v_{\alpha} \\
& =(\tilde{\Lambda}-\mu)(h) u v,
\end{aligned}
$$

onde $\tilde{\Lambda}(h)=\Lambda(h \otimes 1)$. Logo, $V$ é um $\mathcal{G}$-módulo de peso (isto é, admite uma decomposição em espaços de peso sobre $\mathfrak{h} \otimes 1 \cong \mathfrak{h}$ ), onde os pesos de $V$ formam um subconjunto de

$$
\left\{\tilde{\Lambda}-\mu \mid \mu \in \mathcal{Q}_{+}\right\} \subset \mathfrak{h}^{*}
$$

Além disso, o espaço de peso $V_{\tilde{\Lambda}-\mu}=U\left(\mathcal{G}_{-}\right)_{\mu} v$, para todo $\mu \in \mathcal{Q}_{+}$. Como a dimensão de $A$ pode ser infinita, então $V$ pode não ter ser um $\mathcal{G}$-módulo quasi-finito.

Seja $\Lambda \in \mathcal{H}^{*}$, então um $\mathcal{G}$-módulo $M(\Lambda)$ de peso máximo $\Lambda$ e vetor de peso máximo $v_{\Lambda}$ é chamado módulo de Verma (associado a $\Lambda$ ), se para todo $\mathcal{G}$-módulo $V$ de peso máximo $\Lambda$ com vetor de peso máximo $v$ existe um homomorfismo de $\mathcal{G}$-módulos $\psi: M(\Lambda) \rightarrow V$ tal que $\psi\left(v_{\Lambda}\right)=v$.

Utilizando argumentos parecidos aos dados na subseção de módulos de Verma para uma álgebra de Lie semissimples, podemos provar a existência e unicidade de um módulo de Verma com peso 
máximo $\Lambda \in \mathcal{H}^{*}$. Considere o $\mathcal{H} \oplus \mathcal{G}_{+}$-módulo unidimensional $\mathbb{k}_{\Lambda}$ dado por $(h+x) v_{\Lambda}=\Lambda(h) v_{\Lambda}$, para todo $h \in \mathcal{H}$ e $x \in \mathcal{G}_{+}$. Utilizando a notação criada neste capítulo, tome $P=\Phi_{+}$e $\mathcal{P}=\mathcal{H} \oplus \mathcal{G}_{+}$, então

$$
M(\Lambda) \cong M_{\mathcal{P}}\left(\mathbb{k} v_{\Lambda}\right)=U(\mathcal{G}) \otimes_{U(\mathcal{P})} \mathbb{k} v_{\Lambda} .
$$

Aplicando a Proposição 2.2.10 e utilizando a definição dada para módulo de Verma associado à $\Lambda$, temos a seguinte proposição.

Proposição 2.6.3. Seja $\Lambda \in \mathcal{H}^{*}$, então

(i) Como espaços vetoriais,

$$
M(\Lambda) \cong\left(U\left(\mathcal{G}_{-}\right) \mathcal{G}_{-} \otimes \mathbb{k} v_{\Lambda}\right) \oplus \mathbb{k} v_{\Lambda} .
$$

(ii) $M(\Lambda)$ possui um único submódulo próprio maximal $N(\Lambda)$, Em particular, o quociente $L(\Lambda)=$ $M(\Lambda) / N(\Lambda)$ é um $\mathcal{G}$-módulo simples.

(iii) $S e V$ é um $\mathcal{G}$-módulo de peso máximo $\Lambda$, então $V$ possui um único submódulo próprio maximal $N$ e $V / N \cong L(\Lambda)$. Em particular, existe um único $\mathcal{G}$-módulo de peso máximo $\Lambda$ simples.

Observação 2.6.4. A Definição 2.6 .1 poderia ser dada trocando o item 3 por "existe $\lambda \in \mathfrak{h}^{*}$ tal que $(h \otimes 1) v=\lambda(h) v$ e $V_{\lambda}=\{w \in V \mid(h \otimes 1) w=\lambda(h) w\}$ é unidimensional". Neste caso, pelo Teorema de Lie (Teorema 1.2.8), existe $v \in V_{\lambda}$ tal que $U(\mathcal{H}) v=V_{\lambda}$. Como $V_{\lambda}$ é unidimensional, temos que $U(\mathcal{H}) v=\mathbb{k} v$, implicando que existe $\Lambda \in \mathcal{H}^{*}$ tal que $\Lambda(h \otimes 1)=\lambda(h)$ para todo $h \in \mathfrak{h}$ e $(h \otimes a) v=\Lambda(h \otimes a) v$ para todo $h \otimes a \in \mathcal{H}$. Se supuséssemos que $V_{\lambda}$ possui dimensão finita e não necessariamente unidimensional, teríamos, pelo Teorema de Lie, que existe $v \in V_{\lambda}$ não nulo tal que $\mathcal{H} v \subset \mathbb{k} v$. Como $\mathcal{H}$ é uma álgebra de Lie abeliana, a ação de $\mathfrak{h} \otimes 1 \mathrm{em} V_{\lambda}$ é diagonalizável e $U(\mathcal{H}) v=V_{\lambda}$, temos que a ação de $\mathcal{H}$ em $V_{\lambda}$ é diagonalizável se, e somente se, $V_{\lambda}$ possui dimensão 1.

Como foi comentado, se $\Lambda \in \mathcal{H}^{*}$ é qualquer, então $L(\Lambda)$ pode não ser um $\mathcal{G}$-módulo de HarishChandra. Mostraremos uma condição necessária e suficiente para que $L(\Lambda)$ seja um $\mathcal{G}$-módulo de Harish-Chandra e iremos utilizá-la para encontrar um módulo de peso máximo simples que não é um módulo de de Harish-Chandra. Este critério foi baseado ao apresentado em [ERB17] para álgebras correntes de álgebras de Kac-Moody.

Lema 2.6.5. Sejam I um ideal de $A$ e $\Lambda \in \mathcal{H}^{*}$, então $(\mathfrak{g} \otimes I) L(\Lambda)=0$ sse, e somente se, $\Lambda(\mathfrak{h} \otimes I)=$ 0 .

Demonstração. Suponha que $(\mathfrak{g} \otimes I) L(\Lambda)=0$, então, como $(\mathfrak{g} \otimes I) L(\Lambda)=0$ e $(\mathfrak{h} \otimes I) v_{\Lambda}=\Lambda(\mathfrak{h} \otimes I) v_{\Lambda}$, temos que $\Lambda(\mathfrak{h} \otimes I)=0$.

Suponha que $\Lambda(\mathfrak{h} \otimes I)=0$. Seja $\alpha \in \Phi^{+}$, a altura de $\alpha$ é o número inteiro não negativo $\sum_{\alpha \in \Delta} k_{\alpha}$, onde $k_{\alpha}, \alpha \in \Delta$, são inteiros não negativos tais que $\alpha=\sum_{\alpha \in \Delta} k_{\alpha} x_{\alpha}$. Temos que $\alpha \in \Delta$ se, e somente se, a altura de $\alpha$ é 1 . Provaremos, por indução na altura de $\alpha$, que $\left(x_{-\alpha} \otimes I\right) v=0$. Suponha que $\alpha \in \Delta$. Se $\left(x_{\beta} \otimes A\right)\left(x_{-\alpha} \otimes I\right) v_{\Lambda}=0$ para todo $\beta \in \Delta$, então $\left(x_{-\alpha} \otimes I\right) v_{\Lambda}$ gera um submódulo próprio do módulo simples $L(\Lambda)$, implicando que $\left(x_{-\alpha} \otimes I\right) v_{\Lambda}=0$. Portanto, basta mostrar que $\left(x_{\beta} \otimes A\right)\left(x_{-\alpha} \otimes I\right) v_{\Lambda}=0$ para todo $\beta \in \Delta$. Seja $\beta \in \Delta$. Se $\beta \neq \alpha$, então $\left[x_{\beta}, x_{-\alpha}\right]=0$, pois $\beta-\alpha$ não é uma raiz. Como $\left(x_{\beta} \otimes A\right) v_{\Lambda}=0$, então

$$
\begin{aligned}
\left(x_{\beta} \otimes A\right)\left(x_{-\alpha} \otimes I\right) & =\left(x_{-\alpha} \otimes I\right)\left(x_{\beta} \otimes A+\left[x_{\beta}, x_{-\alpha}\right] \otimes I\right) v_{\Lambda} \\
& =\left(x_{-\alpha} \otimes I\right)\left(x_{\beta} \otimes A\right) v_{\Lambda} \\
& =0 .
\end{aligned}
$$

Logo, $\left(x_{-\alpha} \otimes I\right) v_{\Lambda}=0$.

Suponha que a altura de $\alpha$ é maior que 1 . Se $\beta \in \Delta$, então $\left[x_{\beta}, x_{-\alpha}\right] \in \mathfrak{g}_{\beta-\alpha}$. Ou $\alpha-\beta$ é uma raiz positiva com altura menor que $\alpha$, ou $\alpha-\beta$ não é uma raiz. Em ambos os casos, $\left(\left[x_{\beta}, x_{-\alpha}\right] \otimes I\right) v_{\Lambda}=0$, por indução. Logo, como $v_{\Lambda}$ é um vetor de peso máximo,

$$
\left(x_{\beta} \otimes A\right)\left(x_{-\alpha} \otimes I\right) v_{\Lambda}=\left(x_{-\alpha} \otimes I\right)\left(x_{\beta} \otimes A+\left[x_{\beta}, x_{-\alpha}\right] \otimes I\right) v_{\Lambda}
$$




$$
\begin{aligned}
& =\left(x_{-\alpha} \otimes I\right)\left(x_{\beta} \otimes A\right) v_{\Lambda} \\
& =0
\end{aligned}
$$

Temos que $\left(\mathfrak{g}_{+} \otimes I\right) v_{\Lambda}=0$, por definição de vetor de peso máximo, $(\mathfrak{h} \otimes I) v_{\Lambda}=0$, por hipótese, e provamos que $\left(\mathfrak{g}_{-} \otimes I\right) v_{\Lambda}=0$. Logo, $(\mathfrak{g} \otimes I) v_{\Lambda}=0$. Considere o subespaço

$$
W=\{w \in L(\Lambda) \mid(\mathfrak{g} \otimes I) w=0\} .
$$

Portanto, $W$ é não nulo, pois $v_{\Lambda} \in W$. Além disso, $W$ é um submódulo de $L(\Lambda)$, pois $I$ é um ideal de $A$. Como $L(\Lambda)$ é simples, temos que $W=L(\Lambda)$. Logo, $(\mathfrak{g} \otimes I) L(\Lambda)=0$.

Proposição 2.6.6. Seja $\Lambda \in \mathcal{H}^{*}$, então $L(\Lambda)$ é um $\mathcal{G}$-módulo de Harish-Chandra se, e somente se, existe um ideal $I$ de $A$ tal que $\Lambda(\mathfrak{h} \otimes I)=0$ e A/I possui dimensão finita.

Demonstração. Seja $\rho: \mathcal{G} \rightarrow$ End $L(\Lambda)$ a representação associada.

Seja $I$ o ideal de $A$ tal que $\operatorname{ker}(\rho)=\mathfrak{g} \otimes I$. Se $L(\Lambda)$ é um $\mathcal{G}$-módulo de Harish-Chandra, então, pela Proposição 2.1.15, $A / I$ possui dimensão finita. Como $(\mathfrak{g} \otimes I) L(\Lambda)=0$ e $(\mathfrak{h} \otimes I) v_{\Lambda}=\Lambda(\mathfrak{h} \otimes I) v_{\Lambda}$, temos que $\Lambda(\mathfrak{h} \otimes I)=0$.

Seja $I$ um ideal de $A$ tal que $\Lambda(\mathfrak{h} \otimes I)=0$ e $A / I$ possua dimensão finita. Pelo Lema 2.6.5, $(\mathfrak{g} \otimes I) L(\Lambda)=0$. Logo, $\mathfrak{g} \otimes I \subset$ ker $\rho$, portanto existe um único homomorfismo de álgebras de Lie $\tilde{\rho}: \mathfrak{g} \otimes A / I \rightarrow \mathfrak{g l}(L(\Lambda))$ tal que $\tilde{\rho}(a+I)=\rho(a)$, para todo $a \in A$. Note que este homomorfismo preserva os espaços de peso em relação à $\mathfrak{h} \otimes 1$. Sendo assim,

$$
L(\Lambda)=\rho(U(\mathfrak{g} \otimes A)) v_{\Lambda}=\tilde{\rho}(U(\mathfrak{g} \otimes A / I)) v_{\Lambda}=\tilde{\rho}\left(U\left(\mathfrak{g}_{-} \otimes A / I\right)\right) v_{\Lambda} .
$$

Como $\mathfrak{g}_{-} \otimes A / I$ possui dimensão finita, pelo Teorema de Poincaré-Birkhoff-Witt, temos que os espaços de peso de $U\left(\mathfrak{g}_{-} \otimes A / I\right)$ possuem dimensão finita, quando visto como $\mathfrak{h} \otimes 1$-módulo. Logo, os espaços de peso de $L(\Lambda)$ também possuem dimensão finita.

Exemplo 2.6.7 (Um módulo de peso máximo simples que não é um módulo de Harish-Chandra). Seja $\mathfrak{g}=\mathfrak{s l}_{2}$ e $A=\mathbb{C}[t]$. Seja $e, f, h$ a base canônica de $\mathfrak{s l}_{2}$. Defina $\Lambda: \mathbb{C} h \otimes A \rightarrow \mathbb{C}$ por $\Lambda\left(h \otimes t^{k}\right)=k$, então $\operatorname{ker} \Lambda=\mathbb{C} h \otimes J$, onde

$$
J=\left\{\sum_{k=0}^{l} a_{k} t^{k} \in \mathbb{C}[t] \mid \sum_{k=1}^{l} k a_{k}=0, \quad \text { where } l \geq 0\right\} .
$$

Note que $J$ não contém um ideal não nulo de $\mathbb{C}[t]$ : se $J$ contém um ideal não nulo $I$ de $A$, então existe $p(t) \in \mathbb{C}[t]$ tal que $I=\langle p(t)\rangle$, porém existe $s>0$ tal que $t^{s} p(t) \notin J$, implicando que $I$ não é um ideal de $A$. Pela Proposição 2.6.6, $L(\Lambda)$ não é um módulo de Harish-Chandra. Explicitamente, $L(\Lambda)_{\left.\Lambda\right|_{\mathfrak{h} \otimes \mathbb{C}-2}}=\mathbb{C} f \otimes \mathbb{C}[t] v_{\Lambda}$ possui dimensão infinita.

Vimos que $\Phi_{+}$é um conjunto parabólico em $\Phi$. Logo, podemos utilizar a Proposição 2.5.6 para criar $\mathcal{G}$-módulos de peso máximo dados por módulos de avaliação criados a partir de g-módulos de peso máximo e ideais maximais distintos de $A$. Explicitamente, temos a seguinte proposição.

Proposição 2.6.8. Sejam $\lambda_{1}, \ldots, \lambda_{r} \in \mathfrak{h}^{*}, m_{1}, \ldots, m_{r}$ ideais maximais distintos de $A$ e defina $\Lambda \in$ $\mathcal{H}^{*}$ por $\Lambda(h \otimes a)=\sum_{i=1}^{r} a\left(m_{i}\right) \lambda_{i}(h)$, para todo $h \otimes a \in \mathcal{H}$. O $\mathcal{G}$-módulo de avaliação $\bigotimes_{i=1}^{r} L\left(\lambda_{i}\right)\left(m_{i}\right)$ é um $\mathcal{G}$-módulo de Harish-Chandra e $L(\Lambda) \cong \bigotimes_{i=1}^{r} L\left(\lambda_{i}\right)\left(m_{i}\right)$.

Demonstração. Seja $I=\bigcap_{i=1}^{r} m_{r}$. Pelo Teorema dos Zeros de Hilbert e o Teorema Chinês do Resto, $A / I$ possui dimensão finita. Como $\Lambda(A \otimes I)=0$, pela Proposição 2.6.6, $L(\Lambda)$ é um $\mathcal{G}$-módulo de Harish-Chandra.

Sejam $P=\Phi_{+}, \mathfrak{l}=\mathfrak{h}$ e $W_{i}=\mathbb{k} v_{\lambda_{i}}$ o $\mathfrak{h}$-módulo unidimensional tal que $h v_{\lambda_{i}}=\lambda_{i}(h)$, para todo $h \in \mathfrak{h}$. Pela Proposição 2.5.6, $W_{1} \otimes \cdots \otimes W_{r}$ também é unidimensional e

$$
V=\bigotimes_{i=1}^{r} L_{\mathfrak{h} \oplus \mathfrak{g}_{+}}\left(\mathbb{k} v_{\lambda_{i}}\right)\left(m_{i}\right)=\bigotimes_{i=1}^{r} L\left(\lambda_{i}\right)\left(m_{i}\right)
$$


é um $\mathcal{G}$-módulo de Harish-Chandra. Como $V$ é simples, $U(\mathcal{G}) v=V$, onde $v=v_{\lambda_{1}} \otimes \cdots \otimes v_{\lambda_{r}} \in V$. Basta mostrar que $v$ é um vetor de peso máximo $\Lambda$.

Por fim,

$$
(h \otimes a) v=\sum_{i=1}^{r} a\left(m_{i}\right) v_{\lambda_{1}} \otimes \cdots \otimes h v_{\lambda_{i}} \otimes \cdots \otimes v_{\lambda_{r}}=\left(\sum_{i=1}^{r} a\left(m_{i}\right) \lambda_{i}(h)\right) v_{\lambda_{1}} \otimes \cdots \otimes v_{\lambda_{r}},
$$

então $\Lambda \in \mathcal{H}$ é tal que $h v=\Lambda(h) v$, para todo $h \in \mathcal{H}$. Logo, $V$ é um $\mathcal{G}$-módulo simples de peso máximo $\Lambda$, portanto $V \cong L(\Lambda)$.

Corolário 2.6.9. Seja $V$ um $\mathcal{G}$-módulo simples de dimensão finita, então existe $\Lambda \in \mathcal{H}^{*}$ tal que $V \cong L(\Lambda)$, onde $\Lambda \in \mathcal{H}^{*}$ é tal que $\Lambda\left(h_{\alpha} \otimes 1\right) \in \mathbb{Z}$ e $\Lambda\left(h_{\alpha} \otimes 1\right) \geq 0$ para todo $\alpha \in \Delta$. Além disso, existem ideais maximais de $m_{1}, \ldots, m_{r}$ de $A$ e $\lambda_{1}, \ldots, \lambda_{r} \in \mathfrak{h}^{*}$ tais que $\Lambda(h \otimes a)=\sum_{i=1}^{r} a\left(m_{i}\right)$ e $V \cong \bigotimes_{i=1}^{r} L\left(\lambda_{i}\right)\left(m_{i}\right)$.

Demonstração. Temos que $V$ é um $\mathfrak{g} \cong \mathfrak{g} \otimes 1$-módulo de dimensão finita. Pelo Teorema 1.4.1 e Proposição 1.4.3, $V$ é admite decomposição em espaços de peso sobre $\mathfrak{h} \cong \mathfrak{h} \otimes 1$, pois todos os $\mathfrak{g}$ módulos de peso máximo são módulos de peso sobre g. Logo, $V$ é um $\mathcal{G}$-módulo de Harish-Chandra. Pelo Teorema 2.4.7, existem ideais maximais $m_{1}, \ldots, m_{r}$ de $\mathrm{A}$ e $\mathfrak{g}$-módulos simples $W_{1}, \ldots, W_{r}$ tais que

$$
V \cong \bigotimes_{i=1}^{r} W_{i}\left(m_{i}\right)
$$

Como $V$ possui dimensão finita, então $W_{1}, \ldots, W_{r}$ possuem dimensão finita. Pelo Proposição 1.4.3, existem $\lambda_{1}, \ldots, \lambda_{r} \in \mathfrak{h}^{*}$, vetores não nulo de peso $w_{i} \in W_{i}, i=1, \ldots, r$, tais que $h w_{i}=\lambda_{i}(h) w_{i}$, $U\left(\mathfrak{g}_{+}\right) w_{i}=0$ e $U(\mathfrak{g}) v=W$. Isto é, $W_{i}$ é isomorfo ao $\mathfrak{g}$-módulo $L\left(\lambda_{i}\right)$ de peso máximo $\lambda_{i}$. Pelo Teorema 1.4.14, $\lambda_{i}\left(h_{\alpha}\right)$ é um número inteiro não negativo para todo $\alpha \in \Delta$. Seja $\Lambda \in \mathcal{H}^{*}$ dado por $\Lambda(h \otimes a)=\sum_{i}^{r} \lambda_{i}(h) a\left(m_{i}\right)$, então $\Lambda\left(h_{\alpha} \otimes 1\right)=\sum_{i=1}^{r} \lambda_{i}\left(h_{\alpha}\right) \in \mathbb{Z}$ é não negativo para todo $\alpha \in \Delta$ e, pela Proposição 2.6.8,

$$
V \cong \bigotimes_{i=1}^{r} W_{i}\left(m_{i}\right) \cong L(\Lambda)
$$

Na seção anterior, ficamos tentados a mostrar que todo módulo de Harish-Chandra é um módulo de avaliação. Finalizamos este capítulo com um exemplo de que este não é o caso em geral.

Exemplo 2.6.10 (Um módulo de Harish-Chandra que não é um módulo de avaliação). Seja $\mathfrak{g}=\mathfrak{s} \mathfrak{l}_{2}$ e $A=\mathbb{C}[t] /\left(t^{2}\right)$. Seja $e, f, g$ a base canônica de $\mathfrak{s l}_{2}$. Defina $\Lambda: \mathbb{C} h \otimes A \rightarrow \mathbb{C}$ por

$$
\Lambda\left(h \otimes\left(1+\left(t^{2}\right)\right)\right)=\Lambda\left(h \otimes\left(t+\left(t^{2}\right)\right)\right)=1 .
$$

Como $A$ possui dimensão finita, pela Proposição 2.6.6, $L(\Lambda)$ é um módulo de Harish-Chandra. Note que $A$ possui um único ideal maximal e este é o gerado por $t+\left(t^{2}\right)$, portanto todos os módulos de avaliação de $\mathfrak{s l}_{2} \otimes \mathbb{C}[t] /\left(t^{2}\right)$ são da forma $W(t)$, onde $W$ é um $\mathfrak{s l}_{2}$-módulo simples e $\left(x \otimes\left(p(t)+\left(t^{2}\right)\right)\right)=p(0) x v$, para todo $p \in \mathbb{C}[t]$ e $x \in \mathfrak{s l}_{2}$.

Porém, $\left(h \otimes\left(t+\left(t^{2}\right)\right)\right) v_{\Lambda}=\Lambda\left(h \otimes\left(t+\left(t^{2}\right)\right)\right) v_{\Lambda}=v_{\Lambda} \neq 0$, implicando que $L(\Lambda)$ não é um módulo de avaliação. 


\section{Capítulo 3}

\section{Variedades para Álgebra de Takiff Generalizada}

Um famoso teorema de Kostant (veja [Kos63, Theorem 21]) diz que a álgebra universal envolvente de uma álgebra de Lie simples complexa é livre como um módulo sobre seu centro. Como vimos na Seção 1.5, em [FO05], Futorny e Ovsienko construíram uma técnica para provar que uma álgebra especial filtrada $U$ sobre um corpo algebricamente fechado de característica zero é livre como um módulo sobre uma subálgebra comutativa $\Lambda$. Uma álgebra filtrada é chamada de especial filtrada se a álgebra graduada associada é uma álgebra intersecção completa. A técnica consiste em tomar geradores $g_{1}, \ldots, g_{t}$ algebricamente independentes de $\Lambda$ e provar que a imagem deles $\bar{g}_{1}, \ldots, \bar{g}_{t}$ na álgebra graduada $\bar{U}$, associada à filtração de $U$, formam uma sequência regular. Isso é equivalente a mostrar que as componentes irredutíveis de $V\left(\bar{g}_{1}, \ldots, \bar{g}_{t}\right)$ possuem a mesma dimensão $n-t$, onde $n$ é a dimensão de Krull de $\bar{U}$. Dado que $\bar{g}_{1}, \ldots, \bar{g}_{t}$ formam uma sequência regular em $\bar{U}$, temos, pelo teorema principal de [FO05], que $U$ é livre como um módulo à direita (à esquerda) sobre $\Lambda=\mathbb{k}\left[g_{1}, \ldots, g_{t}\right]$. Além disso, por [FO05, Proposition 3.4], para todo ideal maximal $\nu$ de $\Lambda=\mathbb{k}\left[g_{1}, \ldots, g_{t}\right]$, existe um $U$-módulo $M_{\nu}$ irredutível e gerado por $m \in M_{\nu}$ tal que $g m=\chi_{\nu}(g) m$ para todo $g \in \Lambda$, onde $\chi_{\nu}: \Lambda \rightarrow \mathbb{C}$ é o caráter associado à $\nu$.

Pelo Teorema de Poincaré-Birkhoff-Witt, a álgebra universal envolvente de uma álgebra de Lie de dimensão finita é especial filtrada. Aplicaremos a técnica acima em $U=U\left(\mathfrak{g}_{m}(n)\right)$, onde $\mathfrak{g}_{m}(n)=\mathfrak{g l}_{n}(\mathbb{C}) \otimes \mathbb{C}[t] /\left\langle t^{m}\right\rangle$ é a álgebra corrente formada pelo produto tensorial de $\mathfrak{g l}_{n}(\mathbb{C})$ e a álgebra polinomial truncada $\mathbb{C}[t] /\left\langle t^{m}\right\rangle$. Esta álgebra de Lie também é conhecida como álgebra de Takiff generalizada. Consideraremos $\Lambda$ como sendo o centro de $U\left(\mathfrak{g}_{m}(n)\right)$, mas também consideraremos outras subálgebras comutativas como a subálgebra de Gelfand-Tsetlin e a subálgebra de Bethe. Neste capítulo, utilizaremos o Teorema 1.5.29, em conjunto da Proposição 1.5.20 e Lema 1.5.24, para provar que $U\left(\mathfrak{g}_{m}(n)\right)$ é livre como um módulo sobre algumas subálgebras comutativas $\Lambda$. Consideraremos inicialmente $\Lambda$ como sendo o centro de $U\left(\mathfrak{g}_{m}(n)\right)$. Apesar do resultado já ter sido provado, como vimos no Exemplo 1.5.31, vamos, além de construir os geradores algebricamente independentes do centro, mostrar a regularidade destes geradores forma explícita. Também consideraremos a subálgebra de Gelfand-Tsetlin e a subálgebra de Bethe.

Na Seção 3.1, vamos seguir o artigo [Mol97] para construir geradores algebricamende independentes do centro de $U\left(\mathfrak{g}_{m}(n)\right)$. Para isto, precisaremos de alguns resultados relacionados à álgebra Yangian de $\mathfrak{g l}_{n}(\mathbb{C})$ e a álgebra Yangian de level $m$ de $\mathfrak{g l}_{n}(\mathbb{C})$. Em seguida, na Seção 3.2, nosso principal objetivo é mostrar que imagem destes geradores em $\bar{U}\left(\mathfrak{g}_{m}(n)\right)$ são regulares para poder aplicar o teorema principal de [FO05]. O leitor verá que este problema pode ser muito complexo e, por isso, provaremos apenas para $m \leq 2$ ou $n \leq 3$.

Depois disto, consideraremos outra subálgebra comutativa de $U\left(\mathfrak{g}_{m}(n)\right)$, chamada de subálgebra de Gelfand-Tsetlin. Dado $m \geq 1$, existe uma forma natural de definir a cadeia de álgebras de Lie

$$
\mathfrak{g}_{m}(1) \subset \mathfrak{g}_{m}(2) \subset \cdots \subset \mathfrak{g}_{m}(n)
$$


Isto induz uma cadeia nas álgebras universais envolventes

$$
U\left(\mathfrak{g}_{m}(1)\right) \subset U\left(\mathfrak{g}_{m}(2)\right) \subset \cdots \subset U\left(\mathfrak{g}_{m}(n)\right) .
$$

Se $\mathcal{Z}_{m}(n)$ é o centro de $U\left(\mathfrak{g}_{m}(i)\right)$, então a subálgebra $\Gamma_{m}(n)$ de $U\left(\mathfrak{g}_{m}(n)\right)$ gerada por $\mathcal{Z}_{m}(1), \ldots$, $\mathcal{Z}_{m}(n)$ é uma álgebra comutativa chamada de subálgebra de Gelfand-Tsetlin de $U\left(\mathfrak{g}_{m}(n)\right)$. Na Seção 3.3, mostraremos que $\mathcal{V} \mathcal{G} \mathcal{T}_{m}(n)=V\left(\overline{\Gamma_{m}(n)}\right)$ é equidimensional de dimensão $m(n-1) / 2$ se e somente se $n=1$ ou $n=2$, se $m>1$. Isto implicará que $U\left(\mathfrak{g}_{m}(2)\right)$ é livre como um módulo sobre $\Gamma_{m}(2)$. Por fim, consideraremos na Seção 3.4 a subálgebra de $U\left(\mathfrak{g}_{m}(n)\right)$ conhecida como subálgebra de Bethe. Infelizmente conseguimos resultados relevantes apenas quando $n=2$, porém discutimos e exibimos o problema para $U\left(\mathfrak{g}_{m}(3)\right)$.

Os resultados obtidos neste capítulo desta dissertação foram desenvolvidos com a colaboração do Prof. Dr. Germán Benitez Monsalve e foram aceitos para publicação na São Paulo Journal of Mathematical Sciences, doi: 10.1007/s40863-020-00204-1.

Neste capítulo, denotaremos por $\mathbb{C}$ o corpo dos números complexos. Todas as estruturas algébricas, transformações lineares, espaços vetoriais e conjuntos algébricos serão considerados sobre $\mathbb{C}$.

\subsection{Geradores Algebricamente Independentes do Centro}

Seja $\mathfrak{g l}_{n}(\mathbb{C})[t]=\mathfrak{g l}_{n}(\mathbb{C}) \otimes \mathbb{C}[t]$ a álgebra corrente polinomial associada à $\mathfrak{g l}_{n}(\mathbb{C})$. Considere o ideal $I_{m}=\mathfrak{g l}_{n}(\mathbb{C}) \otimes \sum_{k \geq m} t^{k}$ de $\mathfrak{g l}_{n}(\mathbb{C})[t]$ e a álgebra quociente $\mathfrak{g}_{m}=\mathfrak{g}_{m}(n)=\mathfrak{g l}_{n}(\mathbb{C})[t] / I_{m}$. Um dos principais resultados de [RT92] foi a construção de geradores algebricamente independentes da álgebra $I\left(\mathfrak{a}_{m}\right)$ dos $\mathfrak{a}_{m}$-invariantes em $S\left(\mathfrak{a}_{m}\right)$, onde $\mathfrak{a}_{m}=\mathfrak{a} \otimes \mathbb{C}[t] /\left\langle t^{m}\right\rangle$ e $\mathfrak{a}$ é uma álgebra de Lie semissimples. Nesta seção, faremos o mesmo para o centro de $U\left(\mathfrak{g}_{m}(n)\right)$, que é isomorfo, como $\mathfrak{g}_{m^{-}}$ módulo, à álgebra de $\mathfrak{g}_{m}$-invariantes em $S\left(\mathfrak{g}_{m}(n)\right)$ (veja Corolário 1.3.18). Faremos isto seguindo o artigo [Mol97].

Seja $\left\{E_{i j} \mid i, j=1, \ldots, n\right\}$ a base canônica de $\mathfrak{g l}_{n}$ consistindo de matrizes elementares, portanto

$$
\left\{E_{i j}^{(k)}=E_{i j} \otimes t^{k} \mid i, j=1, \ldots, n, k=0, \ldots, m-1\right\}
$$

é uma base de $\mathfrak{g}_{m}(n)$. Considere os polinômios com coeficientes em $U\left(\mathfrak{g}_{m}(n)\right)$ e variável $u$ dados por

$$
E_{i j}(u)=\delta_{i j} u^{m}+\left(E_{i j}^{(0)}-m(j-1) \delta_{i j}\right) u^{m-1}+E_{i j}^{(1)} u^{m-2}+E_{i j}^{(2)} u^{m-3}+\cdots+E_{i j}^{(m-1)},
$$

onde $i, j=1, \ldots, n$.

Seja $\mathfrak{S}_{n}$ o grupo de permutações de $n$ elementos e defina

$$
\operatorname{det} E(u)=\sum_{\sigma \in \mathfrak{S}_{n}} \operatorname{sgn}(\sigma) E_{\sigma(1) 1}(u) E_{\sigma(2) 2}(u) \ldots E_{\sigma(n) n}(u),
$$

chamado de determinante de coluna da matriz não comutativa $\left(E_{i j}(u)\right)=\left(E_{i j}(u)\right)_{i, j=1}^{n}$. Note que $\operatorname{det} E_{i j}(u)$ pode ser visto como um polinômio na variável $u$ e com coeficientes em $U\left(\mathfrak{g}_{m}(n)\right)$. Para $k=1, \ldots, m n$, sejam $\tilde{\zeta}_{k} \in U\left(\mathfrak{g}_{m}\right)$ tais que

$$
\operatorname{det} E(u)=u^{m n}+\sum_{k=1}^{m n} \tilde{\zeta}_{k} u^{m n-k} .
$$

Defina deg' $E_{i j}^{(p)}=p$ e tome $\zeta_{k}$ como sendo a componente de $\tilde{\zeta}_{k}$ com maior "grau" em relação a deg'. Podemos escrever $\zeta_{k}$ explicitamente. Para isto, defina

$$
F_{i j}^{(r)}=E_{i j}^{(r-1)}, 1<r \leq m \text { e } F_{i j}^{(1)}=E_{i j}^{(0)}-2(j-1) \delta_{i j}
$$


Além disso, para cada $k \in\{1, \ldots, m n\}$, sejam $s \in\{1, \ldots, n\}$ e $r \in\{1, \ldots, m n\}$ tais que $k=$ $m(s-1)+r$, então

$$
\zeta_{k}=\sum_{\substack{i_{1}<\cdots<i_{s} \\ j_{1}+\cdots+j_{s}=k}} \sum_{\sigma \in \mathfrak{S}_{s}} \operatorname{sgn}(\sigma) F_{i_{\sigma(1)} i_{1}}^{\left(j_{1}\right)} \cdots F_{i_{\sigma(s)} i_{s}}^{\left(j_{s}\right)}
$$

Exemplo 3.1.1. Vamos supor $n=2$ e $m=2$. Neste caso,

$$
\begin{aligned}
\operatorname{det} E(u)= & E_{11}(u) E_{22}(u)-E_{21}(u) E_{12}(u) \\
= & \left(u^{2}+E_{11}^{(0)} u+E_{11}^{(1)}\right)\left(u^{2}+\left(E_{22}^{(0)}-2\right) u+E_{22}^{(1)}\right)-\left(E_{21}^{(0)} u+E_{21}^{(1)}\right)\left(E_{12}^{(0)} u+E_{12}^{(1)}\right) \\
= & u^{4}+\left(E_{11}^{(0)}+E_{22}^{(0)}-2\right) u^{3}+\left(E_{11}^{(0)}\left(E_{22}^{(0)}-2\right)+E_{11}^{(1)}+E_{22}^{(1)}-E_{21}^{(0)} E_{12}^{(0)}\right) u^{2} \\
& +\left(E_{11}^{(0)} E_{22}^{(1)}+E_{11}^{(1)}\left(E_{22}^{(0)}-2\right)-E_{21}^{(0)} E_{12}^{(1)}-E_{21}^{(1)} E_{12}^{(0)}\right) u+E_{11}^{(1)} E_{22}^{(1)}-E_{21}^{(1)}+E_{12}^{(1)}
\end{aligned}
$$

Portanto, utilizando a notação fixada acima, temos que $\zeta_{1}=F_{11}^{(1)}+F_{22}^{(1)}, \zeta_{2}=F_{11}^{(2)}+F_{22}^{(2)}$,

$$
\begin{gathered}
\zeta_{3}=F_{11}^{(0)} F_{22}^{(1)}+F_{11}^{(1)} F_{22}^{(0)}-F_{21}^{(0)} F_{12}^{(1)}-F_{21}^{(1)} F_{12}^{(0)}, \\
\zeta_{4}=F_{11}^{(1)} F_{22}^{(1)}-F_{21}^{(1)}+F_{12}^{(1)} .
\end{gathered}
$$

O objetivo desta seção é provar parte do principal teorema em [Mol97]:

Teorema 3.1.2. Todos os elementos $\zeta_{k}, k=1, \ldots, m n$, pertencem ao centro $\left.\mathcal{Z}_{m}(n)\right)$ da álgebra $U\left(\mathfrak{g}_{m}(n)\right.$. Além disso, a família $\left\{\zeta_{1}, \zeta_{2}, \ldots, \zeta_{m n}\right\}$ é algebricamente independente e gera $\mathcal{Z}_{m}(n)$.

Para isto, consideraremos a álgebra Yangian da álgebra de Lie $\mathfrak{g l}_{n}(\mathbb{C}$ ) (veja Chapter 1 de [Mol07]), denotada por $Y(n)=Y\left(\mathfrak{g l}_{n}(\mathbb{C})\right)$. Esta álgebra pode ser definida como a álgebra associativa gerada por $t_{i j}^{(1)}, \ldots, t_{i j}^{(m)}, i, j=1, \ldots, n$, sujeita as relações

$$
\left[t_{i j}^{(r)}, t_{i j}^{(s)}\right]=\sum_{a=1}^{\min (r, s)}\left(t_{k j}^{(a-1)} t_{i l}^{(r+s-a)}-t_{k j}^{(r+s-a)} t_{i l}^{(a-1)}\right),
$$

onde $t_{i j}^{(0)}=\delta_{i j}, r, s=1,2, \ldots$ e $1 \leq i, j, k, l \leq n$.

Também consideraremos a álgebra $Y_{m}(n)$ como sendo o quociente de $Y(n)$ pelo ideal $J_{m}$ gerado por todo os elementos $t_{i j}^{(r)}$ com $1 \leq i, j \leq n$ e $r>m$. Esta álgebra é conhecida como Yangian de level $m$ da álgebra de Lie $\mathfrak{g l}_{n}(\mathbb{C})$. Denotaremos por $\tau_{i j}^{(r)}$ a imagem da projeção canônica de $t_{i j}^{(r)}$ em $Y_{m}(n)=Y(n) / J_{m}$, onde $i, j=1, \ldots, n$ e $r>m$. Sendo assim, estes elementos satisfazem as mesmas relações dadas acima. Equivalentemente, $Y_{m}(n)$ pode ser definida pela álgebra gerada por $\tau_{i j}^{(1)}, \ldots, \tau_{i j}^{(m)}, i, j=1, \ldots, n$, sujeita as relações

$$
\left[\tau_{i j}(u), \tau_{k l}(v)\right]=\frac{1}{u-v}\left(\tau_{k j}(u) \tau_{i l}(v)-\tau_{k j}(v) \tau_{i l}(u)\right),
$$

onde $u, v$ são variáveis formais e

$$
\tau_{i j}(u)=\delta_{i j} u^{m}+\sum_{r=1}^{m} \tau_{i j}^{(r)} u^{m-r} \in Y_{m}(n)[u] .
$$

A álgebra Yangian $Y(n)$ pode ser vista como uma deformação da álgebra $U\left(\mathfrak{g l}_{n}[t]\right)$ e, inicialmente, ela foi estudada por causa de sua relação com as soluções da equação de Yang-Baxter quântica. O termo Yangian foi introduzido por Drinfeld no artigo [Dri85], mas a álgebra $Y(n)$ já havia aparecido anteriormente. Neste mesmo artigo, outras álgebras foram definidas e junto de um outro artigo de Jimbo [Jim85], o começo do estudo dos grupos quânticos foi iniciado. A deformação 
que comentamos anteriormente é considerada dentro da categoria das álgebras de Hopf e pode ser feita para álgebras de Lie simples em geral, mas não trataremos destes assuntos neste texto. Caso o leitor esteja interessando por mais informações, veja [Mol07].

Utilizaremos a álgebra $Y_{m}(n)$ e $Y(n)$, pois o centro delas são conhecidos (veja Section 1.7 de [Mol07]), estes podem ser descritos por certos coeficientes de um polinômio chamado determinante quântico. Mostraremos que $Y_{m}(n)$ admite uma filtração cuja álgebra graduada associada é isomorfa a $U\left(\mathfrak{g}_{m}(n)\right)$. Utilizando este isomorfismo, será possível mostrar que a imagem graduada dos elementos geradores do centro de $Y_{m}(n)$ são exatamente os elementos $\zeta_{1}, \ldots, \zeta_{m n}$ construídos acima. Para não estender muito esta seção, utilizaremos resultados relacionados à álgebra Yangian $Y(n)$. Estes resultados podem ser encontrados no livro [Mol07] e disporemos dele como fonte de referência. Começaremos provando uma versão análoga do Teorema de Poincaré-Birkhoff-Witt para a álgebra $Y_{m}(n)$.

Teorema 3.1.3. Aplicando a ordem $\tau_{i j}^{(r)} \leq \tau_{k l}^{(s)}$ se $(r, i, j) \leq(s, k, l)$ na ordem lexicográfica nos geradores de $Y_{m}(n)$, então os monômios

$$
\tau_{i_{1} j_{1}}^{\left(r_{1}\right)} \cdots \tau_{i_{q} j_{q}}^{\left(r_{q}\right)}, \quad \tau_{i_{1} j_{1}}^{\left(r_{1}\right)} \leq \cdots \leq \tau_{i_{q} j_{q}}^{\left(r_{q}\right)}
$$

formam uma base de $Y_{m}(n)$.

Demonstração. Lembre que $Y_{m}(n)=Y(n) / J_{m}$. Portanto, podemos considerar a mesma ordem nos geradores $t_{i j}^{(r)}$ e mostrar o teorema para a ordem induzida em $\tau_{i j}^{(r)}$. Considere a ordem $t_{i j}^{(r)} \leq t_{k l}^{(s)}$ se $(r, i, j) \leq(s, k, l)$ na ordem lexicográfica. Pelo Teorema de Poincaré-Birkhoff-Witt para a álgebra Yangian $Y(n)$ (veja Theorem 1.4.1 de [Mol07]), os polinômios ordenados

$$
t_{i_{1} j_{1}}^{\left(r_{1}\right)} \cdots t_{i_{q} j_{q}}^{\left(r_{q}\right)} \quad t_{i_{1} j_{1}}^{\left(r_{1}\right)} \leq \cdots \leq t_{i_{q} j_{q}}^{\left(r_{q}\right)}
$$

formam uma base de $Y(n)$. Temos que cada elemento de $J_{m}$ é uma combinação linear de monômios da forma (3.3). Mostraremos que cada monômio nesta representação é tal que $r_{q}>m$. Como espaço vetorial, $J_{m}$ é gerado por monômios da forma

$$
t_{k_{1} l_{1}}^{\left(s_{1}\right)} \cdots t_{k_{p} l_{p}}^{\left(s_{p}\right)}, \quad p \geq 1
$$

onde pelo menos um dos índices $s_{1}, \ldots, s_{p}$ é maior que $m$.

Em particular, se $p=2$, utilizando as relações (3.1), temos que, para cada par $t_{i j}^{(r)} \geq t_{k l}^{(s)}$,

$$
\begin{aligned}
t_{i j}^{(r)} t_{k l}^{(s)} & =t_{i j}^{(s)} t_{k l}^{(r)}+\left[t_{k l}^{(r)}, t_{i j}^{(s)}\right] \\
& =t_{i j}^{(s)} t_{k l}^{(r)}+\sum_{a=1}^{s}\left(t_{k j}^{(a-1)} t_{i l}^{(r+s-a)}-t_{k j}^{(r+s-a)} t_{i l}^{(a-1)}\right)
\end{aligned}
$$

Utilizando indução em $r+s$ e supondo $r>m$, temos que

$$
t_{i j}^{(r)} t_{k l}^{(s)}=t_{i j}^{(s)} t_{k l}^{(r)}+\text { combinação linear de } t_{a b}^{(p)} t_{c d}^{(q)} \operatorname{com} t_{a b}^{(p)}<t_{c d}^{(q)}, p+q<r+s \text { e } q>m .
$$

Suponha que provamos o resultado para $p>2$. Fixe $t_{k_{1} l_{1}}^{\left(s_{1}\right)} \cdots t_{k_{p} l_{p}}^{\left(s_{p}\right)} \in J_{m}$. Seja $i \in\{1, \ldots, p\}$ tal que $t_{k_{i} l_{i}}^{\left(s_{i}\right)} \geq t_{k_{j} l_{j}}^{\left(s_{j}\right)}$, para todo $j=1, \ldots, p$. Note que $s_{i}>m$. Utilizando um argumento estendido ao dado no paragrafo anterior, podemos comutar $t_{k_{i} l_{i}}^{\left(s_{i}\right)}$ com os outros geradores e movê-lo para a extrema direita do monômio. Assim, teremos que $t_{k_{1} l_{1}}^{\left(s_{1}\right)} \cdots t_{k_{p} l_{p}}^{\left(s_{p}\right)}$ é igual à

$$
\begin{array}{r}
t_{k_{1} l_{1}}^{\left(s_{1}\right)} \cdots t_{k_{i-1} l_{i-1}}^{\left(s_{i-1}\right)} t_{k_{i+1} l_{i+1}}^{\left(s_{i+1}\right)} \cdots t_{k_{p} l_{p}}^{\left(s_{p}\right)} t_{k_{i} l_{i}}^{\left(s_{i}\right)}+ \\
\text { combinação linear de } t_{a_{1} b_{1}}^{\left(c_{1}\right)} \cdots t_{a_{p} b_{p}}^{\left(c_{p}\right)} \\
\operatorname{com~} c_{1}+\cdots+c_{p}<s_{1}+\cdots+s_{p},
\end{array}
$$


Repetindo este processo, temos que $t_{k_{1} l_{1}}^{\left(s_{1}\right)} \cdots t_{k_{p} l_{p}}^{\left(s_{p}\right)}$ é igual a $t_{k_{\sigma(1)} l_{\sigma(1)}}^{\left(s_{\sigma(1)}\right)} \cdots t_{k_{\sigma(p)} l_{\sigma(p)}}^{\left(s_{\sigma(p)}\right)}$ mais uma combinação linear de $t_{a_{1} b_{1}}^{\left(c_{1}\right)} \cdots t_{a_{p} b_{p}}^{\left(c_{p}\right)}$ com $c_{1}+\cdots+c_{p}<s_{1}+\cdots+s_{p}$, onde $t_{k_{\sigma(1)} l_{\sigma(1)}}^{\left(s_{\sigma(1)}\right)} \leq \cdots \leq t_{k_{\sigma(p)} l_{\sigma(p)}}^{\left(s_{\sigma(p)}\right)}$, $\sigma \in \mathfrak{S}_{p}, \sigma(p)=i$. Utilizando indução em $s_{1}+\cdots+s_{n}$, temos que $t_{k_{1} l_{1}}^{\left(s_{1}\right)} \cdots t_{k_{p} l_{p}}^{\left(s_{p}\right)}$ é combinação linear de monômios da forma (3.3) tal que $r_{q}>m$. Como $Y_{m}(n)=Y(n) / J_{m}$, os monômios

$$
\tau_{i_{1} j_{1}}^{\left(r_{1}\right)} \cdots \tau_{i_{q} j_{q}}^{\left(r_{q}\right)}, \quad \tau_{i_{1} j_{1}}^{\left(r_{1}\right)} \leq \cdots \leq \tau_{i_{q} j_{q}}^{\left(r_{q}\right)}, \quad r_{q} \leq m
$$

formam uma base de $Y_{m}(n)$.

Podemos definir uma filtração em $Y_{m}(n)$ definido

$$
\operatorname{deg} \tau_{i j}^{(r)}=r-1
$$

Denote por $\bar{Y}_{m}(n)$ a álgebra graduada associada a esta filtração.

Corolário 3.1.4. A álgebra $\bar{Y}_{m}(n)$ é isomorfa à $U\left(\mathfrak{g}_{m}(n)\right)$.

Demonstração. Denote por $\bar{\tau}_{i j}^{(r)}$ a imagem de $\tau_{i j}^{(r)}$ no espaço homogêneo de grau $r-1$ em $\bar{Y}_{m}(n)$. Comparando o grau dos elementos em (3.1), temos que

$$
\left[\tau_{i j}^{(r)}, \tau_{i j}^{(s)}\right]=\delta_{k j} \tau_{i l}^{(r+s-1)}-\delta_{i l} \tau_{k j}^{(r+s-1)},
$$

definindo $\tau_{i j}^{(r)}=0$, para $r>m$. Considere a aplicação linear induzida por

$$
\begin{aligned}
\varphi: U\left(\mathfrak{g}_{m}(n)\right) & \longrightarrow \bar{Y}_{m}(n) \\
E_{i j}^{(r-1)} & \longmapsto \tau_{i j}^{(r)},
\end{aligned}
$$

então

$$
\begin{aligned}
\varphi\left(\left[E_{i j}^{(r-1)}, E_{k l}^{(s-1)}\right]\right) & =\varphi\left(\left[E_{i j}, E_{k l}\right] \otimes x^{r+s-2}\right) \\
& =\delta_{k l} \varphi\left(E_{i l}^{(r+s-2)}\right)-\delta_{i l} \varphi\left(E_{k j}^{(r+s-2)}\right) \\
& =\delta_{k j} \tau_{i l}^{(r+s-1)}-\delta_{i l} \tau_{k j}^{(r+s-1)} \\
& =\left[\tau_{i j}^{(r)}, \tau_{i j}^{(s)}\right] \\
& =\left[\varphi\left(E_{i j}^{(r-1)}\right), \varphi\left(E_{k l}^{(s-1)}\right)\right] .
\end{aligned}
$$

Portanto, $\varphi$ induz um homomorfismos de álgebras associativas. Considere a ordem na base canônica de $\mathfrak{g}_{m}(n) E_{i j}^{(r)} \leq E_{k l}^{(s)}$ se $(l, i, j) \leq(s, k, l)$ na ordem lexicográfica. Pelo Teorema de Poincaré-Birkhoff-Witt para a álgebra $U\left(\mathfrak{g}_{m}(n)\right)$ temos que os monômios

$$
E_{i_{1} j_{1}}^{\left(r_{1}\right)} \cdots E_{i_{q} j_{q}}^{r_{q}}, \quad E_{i_{1} j_{1}}^{\left(r_{1}\right)} \leq \cdots \leq E_{i_{q} j_{q}}^{r_{q}}
$$

formam uma base de $U\left(\mathfrak{g}_{m}(n)\right)$. Sendo assim, pelo Teorema 3.1.3, $\varphi$ é um homomorfismo de álgebras que leva uma base de $U\left(\mathfrak{g}_{m}(n)\right)$ para uma base de $\bar{Y}_{m}(n)$, implicando que $\varphi$ é um isomorfismo de álgebras associativas.

Lema 3.1.5. Os elementos $\zeta_{1}, \ldots, \zeta_{m n}$ pertencem ao centro de $U\left(\mathfrak{g}_{m}(n)\right)$. 
Demonstração. Como fizemos com a base de $U\left(\mathfrak{g}_{m}(n)\right)$, considere a matriz $\mathcal{T}(u)=\left(\tau_{i j}^{(u)}\right)_{i, j=1}^{n} \operatorname{com}$ coeficientes em $Y_{m}(n)[u]$. O polinômio

$$
\text { qdet } \mathcal{T}(u)=\sum_{\sigma \in \mathfrak{S}_{n}} \operatorname{sgn}(p) \tau_{\sigma(1) 1}(u) \tau_{\sigma(2) 2}(u+1) \ldots \tau_{\sigma(n) n}(u-n+1)
$$

é chamado de determinante quântico da matriz $\mathcal{T}(u)$ (veja Section 1.6 de [Mol07]).

Sejam $d_{1}, \ldots, d_{k} \in Y_{m}(n)$ tais que

$$
\text { qdet } \mathcal{T}(u)=u^{m n}+\sum_{k=1}^{m n} d_{k} u^{m n-k},
$$

então os elementos $d_{1}, \ldots, d_{m n}$ pertencem ao centro de $Y_{m}(n)$. Isto é consequência do fato de que tais coeficientes da versão do determinante quântico para o Yangian $Y(n)$ pertencem ao centro dele (veja Theorem 1.7.5 de [Mol07]). Sendo assim, as imagens $\bar{d}_{1}, \ldots, \bar{d}_{m n}$ também pertencem ao centro de $\bar{Y}_{m}(n)$.

Demonstraremos que a imagem de $\zeta_{k}$ no isomorfismo construído no Corolário 3.1.4 é $\bar{d}_{k}$. Para isto, basta ver que a imagem do polinômio

$$
\tau_{i j}(u-j+1)=\delta_{i j}(u+j-1)^{m}+\sum_{k=1}^{m} \tau_{i j}^{(k)}(u-j+1)^{m-k}
$$

em $\bar{Y}_{m}(n)[u]$ é

$$
\delta_{i j} u^{m}+\left(\bar{\tau}_{i j}^{(1)}-m(j-1) \delta_{i j}\right) u^{m-1}+\sum_{k=2}^{m} \tau_{i j}^{(k)} u^{m-k} .
$$

Utilizando o isomorfismo construído no Corolário 3.1.4, temos que este corresponde à imagem de $E_{i j}(u)$. Logo, a imagem de $\zeta_{k}$ no isomorfismo construído no Corolário 3.1.4 é $\bar{d}_{k}$.

Lema 3.1.6. Os elementos $\zeta_{1}, \ldots, \zeta_{m n} \in U\left(\mathfrak{g}_{m}(n)\right)$ são algebricamente independentes.

Demonstração. Basta mostrar que a imagem graduada $\bar{\zeta}_{1}, \ldots, \bar{\zeta}_{m n} \in \bar{U}\left(\mathfrak{g}_{m}(n)\right)$ de $\zeta_{1}, \ldots, \zeta_{m n}$ é algebricamente independente. Como cada $\bar{\zeta}_{k}$ pode ser visto como uma função polinomial em $\mathbb{C}^{m n^{2}}$ com variáveis

$$
\left\{\bar{F}_{i j}^{(l)} \mid l=1, \ldots, m ; i, j=1, \ldots n\right\}
$$

pelo critério do Jacobiano para independência algébrica (veja [ER93, Theorem 2.3]), é suficiente mostrar que o conjunto das suas diferenciais

$$
\left\{d\left(\bar{\zeta}_{k}\right): \mathbb{C}^{m n^{2}} \rightarrow\left(\mathbb{C}^{m n}\right)^{*} \mid k=1, \ldots, m n\right\}
$$

é linearmente independente. Considere a base

$$
B=\left\{\mathbf{e}_{i j}^{(l)} \mid l=1, \ldots, m ; i, j=1, \ldots n\right\}
$$

de $\mathbb{C}^{m n^{2}}$, sua base dual $B^{*}=\left\{f_{i j}^{(l)} \mid l=1, \ldots, m ; i, j=1, \ldots n\right\} \subset\left(\mathbb{C}^{m n^{2}}\right)^{*}$ e o ponto $v \in \mathbb{C}^{m n^{2}}$, dado por

$$
f_{i j}^{(l)}(v)= \begin{cases}1 & \text { se } i=2, \ldots, n-1, j=i-1, l=n \\ 0 & \text { caso contrário. }\end{cases}
$$

Mostraremos que o conjunto $\left\{\eta_{k} \mid k=1, \ldots, m n\right\}$ é linearmente independente, onde

$$
\eta_{k}=d\left(\bar{\zeta}_{k}\right)(v) \in\left(\mathbb{C}^{m\left(n^{2}-n\right)}\right)^{*} .
$$


Pela definição de $\bar{\zeta}_{k}$, deduzimos que para $k=1, \ldots, m$

$$
\eta_{k}=\sum_{i=1}^{n} f_{i i}^{(k)}
$$

Além disso, se $s \in\{1, \ldots, m\}$ e $r \in\{1, \ldots, m\}$ são tais que $k=m(s-1)+r$, então, para $k=m+1, m+2, \ldots, m n$,

$$
\eta_{k}=\sum_{i=1}^{n-s+1} f_{i, i+s-1}^{(r)}
$$

Como

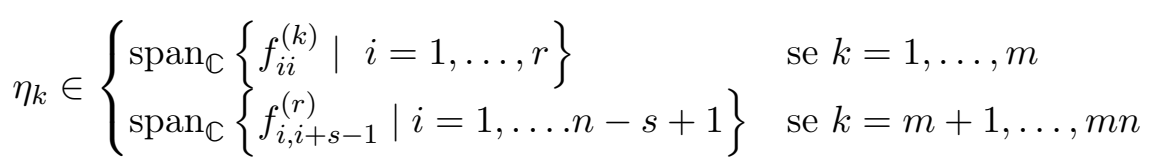

e esses espaços possuem intersecção nula, temos que $\eta_{1}, \ldots, \eta_{m n}$ é linearmente independente. Logo, $\left\{d\left(\bar{\zeta}_{k}\right) \mid k=1, \ldots, m n\right\}$ é linearmente independente.

Lema 3.1.7. Os elementos $\zeta_{1}, \ldots, \zeta_{m n} \in U\left(\mathfrak{g}_{m}(n)\right)$ geram o centro de $U\left(\mathfrak{g}_{m}(n)\right)$.

Demonstração. Pelo Teorema de Poincaré-Birkhoff-Witt, a álgebra $U\left(\mathfrak{g}_{m}(n)\right)$ é uma álgebra filtrada, tomando $\operatorname{deg} E_{i j}^{(l)}=1$, cuja álgebra graduada associada é isomorfa à álgebra simétrica $S\left(\mathfrak{g}_{m}(n)\right)$. A representação adjunta torna $S\left(\mathfrak{g}_{m}(n)\right)$ um $\mathfrak{g}_{m}(n)$-módulo. Considere a álgebra $I\left(\mathfrak{g}_{m}(n)\right)$ de $\mathfrak{g}_{m}(n)$-invariantes de $S\left(\mathfrak{g}_{m}(n)\right)$, isto é,

$$
I\left(\mathfrak{g}_{m}(n)\right)=\left\{f \in S\left(\mathfrak{g}_{m}(n)\right) \mid x \cdot f=0 \text { para todo } x \in \mathfrak{g}\right\},
$$

onde $x \cdot f$ é a ação induzida pela representação adjunta. Pelo Corolário 1.3.18, o isomorfismo entre $\bar{U}\left(\mathfrak{g}_{m}(n)\right)$ e $S\left(\mathfrak{g}_{m}(n)\right)$ define um isomorfismo entre $I\left(\mathfrak{g}_{m}(n)\right)$ e $\overline{Z_{m}}$, onde $Z_{m}$ é o centro de $U\left(\mathfrak{g}_{m}(n)\right)$.

Para cada $k=1, \ldots, m n$, denote por $\bar{\zeta}_{k}$ a imagem de $\zeta_{k}$ em $\bar{U}\left(\mathfrak{g}_{m}(n)\right)$. Pelo Théorème 4.5 de [RT92], $I\left(\mathfrak{g}_{m}(n)\right)$ admite geradores $P_{r s}, 1 \leq r \leq m, 1 \leq s \leq n$, algebricamente independentes e homogêneos, onde $P_{r s}$ possui grau $s$. Como $\bar{\zeta}_{1}, \ldots, \bar{\zeta}_{m n}$ são algebricamente independentes e homogêneos, pelo isomorfismo entre $I\left(\mathfrak{g}_{m}(n)\right)$ e $\bar{Z}_{m}$, basta mostrar que $\bar{\zeta}_{1}, \ldots, \bar{\zeta}_{m n}$ possuem o mesmo grau que os geradores $P_{r s}, r=1, \ldots, m, s=1, \ldots, n$. Vendo a definição de $\bar{\zeta}_{k}$, fica claro que $\bar{\zeta}_{k}$ possui grau $s$, onde $k=m(s-1)+r$, tal que $s=1, \ldots, n$ e $r=1, \ldots, m$.

Sendo assim, pelo Lema 3.1.5, Lema 3.1.6 e Lema 3.1.7, temos a prova do Teorema 3.1.2.

\subsection{Teorema de Kostant}

Pelo Teorema de Poincaré-Birkhoff-Witt, a álgebra graduada $\bar{U}\left(\mathfrak{g}_{m}(n)\right)$ é a álgebra polinomial nas variáveis $\bar{E}_{i j}^{(k)}$, o que implica que $U\left(\mathfrak{g}_{m}(n)\right)$ é uma álgebra especial filtrada. Sejam $\bar{\zeta}_{1}, \ldots, \bar{\zeta}_{m n}$ a imagem graduada de $\zeta_{1}, \ldots, \zeta_{m n}$, respectivamente, então

$$
\bar{\zeta}_{k}=\sum_{\substack{i_{1}<\cdots<i_{s} \\ j_{1}+\cdots+j_{s}=k}} \sum_{\sigma \in \mathfrak{S}_{s}} \operatorname{sgn}(\sigma) \bar{F}_{i_{\sigma(1)} i_{1}}^{\left(j_{1}\right)} \cdots \bar{F}_{i_{\sigma(s)} i_{s}}^{\left(j_{s}\right)},
$$

onde $k=m(s-1)+r$, onde $s \in\{1, \ldots, n\}$ e $r \in\{1, \ldots, m\}$. Apesar de estarmos escrevendo $\bar{\zeta}_{1}, \ldots, \bar{\zeta}_{m n}$ em termos de $\bar{F}_{i j}^{l}$, eles são elementos da álgebra polinomial

$$
\mathbb{C}\left[\bar{E}_{i j}^{(k)} \mid i, j=1, \ldots, n, k=0, \ldots, m-1\right],
$$

pois $\bar{F}_{i j}^{(l)}=\bar{E}_{i j}^{(l-1)}$. 
Ressaltamos, novamente, que a maior parte da notação e resultados que utilizaremos está compilado na Seção 1.5. Recomendamos que o leitor reveja os resultados, definições e notações dessa seção. Em especial, Proposição 1.5.20, Lema 1.5.24, Notação 1.5.25 e Teorema 1.5.29.

O lema a seguir é análogo ao Teoreoma de Kostant para $\mathfrak{g}_{m}(1)$ e $\mathfrak{g}_{1}(n)$, onde $m, n \geq 1$. Além disso, a demonstração do lema é um bom exemplo de como será nossa estratégia para provar que $U\left(\mathfrak{g}_{m}(n)\right)$ é livre como módulo sobre alguma subálgebra comutativa dada, como o seu próprio centro, a subálgebra de Gelfand-Tsetlin e a subálgebra de Bethe.

Lema 3.2.1. As álgebras $U\left(\mathfrak{g}_{m}(1)\right)$ e $U\left(\mathfrak{g}_{1}(n)\right)$ são livres como módulos sobre seus centros, onde $m, n \geq 1$.

Demonstração. Começaremos considerando $\mathfrak{g}_{m}(1)$. Como $\bar{\zeta}_{i}=\bar{F}_{11}^{(i)}$ para todo $i=1, \ldots, m$, segue da Proposição 1.5.20 que $\bar{\zeta}_{1}, \ldots, \bar{\zeta}_{m}$ é uma sequência regular em $\mathbb{C}\left[\bar{F}_{11}^{(1)}, \ldots, \bar{F}_{11}^{(m)}\right]$, pois

$$
V\left(\bar{\zeta}_{1}, \ldots, \bar{\zeta}_{m}\right)=V\left(\bar{F}_{11}^{(1)}, \ldots, \bar{F}_{11}^{(m)}\right)=\{0\} .
$$

Logo, $U\left(\mathfrak{g}_{m}(1)\right)$ é livre como um módulo sobre seu centro pelo Teorema 1.5.29.

Agora, considere $\mathfrak{g}_{1}(n)$. Queremos provar que $\bar{\zeta}_{1}, \ldots, \bar{\zeta}_{n}$ é uma sequência regular em

$$
\mathbb{C}\left[\bar{F}_{i j}^{(l)} \mid i, j=1, \ldots, n\right] .
$$

Pela Proposição 1.5.20, basta mostrar que $V\left(\bar{\zeta}_{1}, \ldots, \bar{\zeta}_{n}\right) \subset \mathbb{C}^{n^{2}}$ é equidimensional de dimensão $n^{2}-n$. Segue da Proposição 1.5.20 que é suficiente mostrar que o conjunto algébrico

$$
V\left(\left\{\bar{\zeta}_{i}\right\}_{i=1}^{n} \cup \mathbf{X}\right) \subset \mathbb{C}^{n^{2}} \operatorname{com} \mathbf{X}=\left\{\bar{F}_{i j}^{(1)} \mid i, j=1, \ldots, n ; i \neq j\right\}
$$

é equidimensional de dimensão zero. Pelo Lema 1.5.24, isto é equivalente mostrar que

$$
V\left(\bar{\zeta}_{1}^{\mathbf{X}}, \ldots, \bar{\zeta}_{n}^{\mathbf{X}}\right) \subset \mathbb{C}^{n^{2}-\left(n^{2}-n\right)}=\mathbb{C}^{n}
$$

é equidimensional de dimensão zero (veja Notação 1.5.25). Como

$$
\bar{\zeta}_{k}^{\mathbf{X}}=\sum_{i_{1}<\cdots<i_{k}} \bar{F}_{i_{1} i_{1}}^{(1)} \ldots \bar{F}_{i_{k} i_{k}}^{(1)}
$$

é o polinômio elementar simétrico de grau $k$ nas $n$ variáveis $\bar{F}_{11}^{(1)}, \ldots, \bar{F}_{n n}^{(1)}$, segue, pela Proposição 1.5.26, que

$$
V\left(\bar{\zeta}_{1}^{\mathbf{X}}, \ldots, \bar{\zeta}_{n}^{\mathbf{X}}\right)=\{0\}
$$

é uma variedade algébrica de dimensão zero. Logo, $\bar{\zeta}_{1}, \ldots, \bar{\zeta}_{n}$ é uma sequência regular em $\mathbb{C}\left[\bar{F}_{i j} \mid\right.$ $i, j=1, \ldots, n]$ e, pelo Teorema 1.5.29, temos que $U\left(\mathfrak{g}_{m}(n)\right)$ é livre como um módulo sobre seu centro.

Se $\mathfrak{a}$ é uma álgebra de Lie simples de dimensão finita sobre $\mathbb{C}$, a álgebra $\hat{\mathfrak{a}}=\mathfrak{a} \otimes \mathbb{C}[t] /\left\langle t^{2}\right\rangle$ é chamada de álgebra de Takiff (veja [Geo94b], [Geo94a]). Foi provado em [Geo94b] que $U(\hat{\mathfrak{a}})$ é livre como um módulo sobre seu centro. Isto é equivalente à mostrar que $U\left(\mathfrak{g}_{2}(n)\right)$ é livre como módulo sobre seu centro, no caso particular de $\mathfrak{g}=\mathfrak{s l}_{n}(\mathbb{C})$. Provaremos de forma diferente este resultado, utilizando a mesma técnica da prova anterior. Para isto, precisaremos da seguinte proposição.

Proposição 3.2.2 ( [Ben20, Theorem 3.6]). O conjunto algébrico

$$
V\left(\sigma_{n 2}, \sigma_{n 3}, \ldots, \sigma_{n n}\right) \subset \mathbb{C}^{\frac{(n+2)(n-1)}{2}}
$$


é equidimensional de dimensão $n(n-1) / 2$, onde

$$
\sigma_{n s}=\sum_{n>t_{1}>\cdots>t_{s-1} \geq 1} X_{n t_{1}} X_{t_{1} t_{2}} \cdots X_{t_{s-2} t_{s-1}} X_{t_{s-1} n}, \quad s=2,3, \ldots, n
$$

Teorema 3.2.3. A álgebra $U\left(\mathfrak{g}_{2}(n)\right)$ é livre como um módulo sobre seu centro.

Demonstração. Primeiramente, mostraremos que

$$
\bar{\zeta}_{1}, \ldots, \bar{\zeta}_{2 n}
$$

é uma sequência regular em $\bar{U}\left(\mathfrak{g}_{2}(n)\right)$. Pela Proposição 1.5.20, é suficiente mostrar que o conjunto algébrico

$$
\mathcal{V}:=V\left(\left\{\bar{F}_{i j}^{(1)}\right\}_{\substack{1 \leq i \leq n-1 \\ 1 \leq j \leq n}} \cup\left\{\bar{F}_{i j}^{(2)}\right\}_{1 \leq i<j \leq n} \cup\left\{\bar{\zeta}_{s}\right\}_{s=1}^{2 n}\right) \subset \mathbb{C}^{2 n^{2}}
$$

é equidimensional de dimensão $n(n-1) / 2$. Tome $\mathbf{X}=\left\{\bar{F}_{i i}^{(1)}\right\}_{i=1}^{n-1}$ e $\mathbf{Y}=\left\{\bar{F}_{i j}^{(2)}\right\}_{1 \leq i<j \leq n}$, então

$$
\bar{\zeta}_{1}^{\mathbf{X}}=\bar{F}_{n n}^{(1)} \quad \text { e } \quad \bar{\zeta}_{2 t}^{\mathbf{Y}}=\sum_{i_{1}<\cdots<i_{t}} \bar{F}_{i_{1} i_{1}}^{(2)} \cdots \bar{F}_{i_{t} i_{t}}^{(2)} .
$$

Sendo assim, $\bar{\zeta}_{2 t}^{\mathbf{Y}}$ é o polinômio simétrico de grau $t$ nas $n$ variáveis $\bar{F}_{11}^{(2)}, \ldots, \bar{F}_{n n}^{(2)}$, para cada $t=$ $1, \ldots, n$. Isto implica que $\mathcal{V}=V\left(\mathbf{A} \cup\left\{\bar{\zeta}_{2 s-1}\right\}_{s=2}^{n}\right)$, onde

$$
\mathbf{A}:=\left\{\bar{F}_{i j}^{(1)}\right\}_{\substack{1 \leq i \leq n-1 \\ 1 \leq j \leq n}} \cup\left\{\bar{F}_{n n}^{(1)}\right\} \cup\left\{\bar{F}_{i j}^{(2)}\right\}_{1 \leq i \leq j \leq n} .
$$

Considere os polinômios $\Lambda_{s}^{n}:=(-1)^{(s+1)} \bar{\zeta}_{2 s-1}^{\mathbf{A}}$ para $s=2,3, \ldots, n$. Pela definição de $\bar{\zeta}_{2 s-1}$, cada polinômio $\Lambda_{s}^{n}$ é a soma de todos os monômios da forma $\bar{F}_{i_{2} i_{1}}^{(2)} \bar{F}_{i_{3} i_{2}}^{(2)} \cdots \bar{F}_{n i_{s-1}}^{(2)} \bar{F}_{i_{1} n}^{(1)} \operatorname{com} i_{1}<$ $\cdots<i_{s-1}<n$. Portanto,

$$
\Lambda_{s}^{n}=\sum_{i_{1}<\cdots<i_{s-1}<n} \bar{F}_{i_{2} i_{1}}^{(2)} \bar{F}_{i_{3} i_{2}}^{(2)} \cdots \bar{F}_{n i_{s-1}}^{(2)} \bar{F}_{i_{1} n}^{(1)}=\sum_{n>t_{1}>\cdots>t_{s-1} \geq 1} \bar{F}_{n t_{1}}^{(2)} \bar{F}_{t_{1} t_{2}}^{(2)} \cdots \bar{F}_{t_{s-2} t_{s-1}}^{(2)} \bar{F}_{t_{s-1} n}^{(1)} .
$$

Pelo Lema 1.5.24, só precisamos mostrar que $V\left(\Lambda_{2}^{n}, \ldots, \Lambda_{n}^{n}\right) \subset \mathbb{C}^{\frac{(n+2)(n-1)}{2}}$ é equidimensional de dimensão $n(n-1) / 2$, mas isto é uma consequência da mudança de variável $X_{i j} \longleftrightarrow \bar{F}_{i j}^{(2)}$ (onde $j \neq n)$ e $X_{i n} \longleftrightarrow \bar{F}_{i n}^{(1)}$ e a Proposição 3.2.2.

Como $\mathcal{V}$ é equidimensional de dimensão $n(n-1) / 2$, segue da Proposição 1.5.20 que $\bar{\zeta}_{1}, \bar{\zeta}_{2}, \ldots, \bar{\zeta}_{2 n}$ é uma sequência regular em $\bar{U}\left(\mathfrak{g}_{2}(n)\right)$. Pelo Teorema 1.5.29, $U\left(\mathfrak{g}_{2}(n)\right)$ é livre como um módulo sobre seu centro.

Lema 3.2.4. Suponha $n=2$. Para $m>1, V\left(\bar{\zeta}_{m+1}^{\boldsymbol{X}}, \ldots, \bar{\zeta}_{2 m}^{\boldsymbol{X}}\right) \subset \mathbb{C}^{2 m}$ é equidimensional de dimensão $m$, onde $\boldsymbol{X}=\left\{\bar{F}_{i i}^{(l)} \mid i=1,2 ; l=1, \ldots, m\right\}$.

Demonstração. Provaremos utilizando indução em $m$ que $V_{m}=V\left(\bar{\zeta}_{m+1}^{\mathbf{X}}, \ldots, \bar{\zeta}_{2 m}^{\mathbf{X}}\right)$ é equidimensional de dimensão $m$. Se $m=2$, então $\bar{\zeta}_{4}^{\mathbf{X}}=\bar{F}_{21}^{(2)} \bar{F}_{12}^{(2)}$ e $\bar{\zeta}_{3}^{\mathbf{X}}=\bar{F}_{21}^{(1)} \bar{F}_{12}^{(2)}+\bar{F}_{21}^{(2)} \bar{F}_{12}^{(1)}$. Logo,

$$
\begin{aligned}
V\left(\bar{\zeta}_{4}^{\mathbf{X}}, \bar{\zeta}_{3}^{\mathbf{X}}\right) & =V\left(\bar{F}_{21}^{(2)} \bar{F}_{12}^{(2)}, \bar{F}_{21}^{(1)} \bar{F}_{12}^{(2)}+\bar{F}_{21}^{(2)} \bar{F}_{12}^{(1)}\right) \\
& =V\left(\bar{F}_{21}^{(2)}, \bar{F}_{21}^{(2)} \bar{F}_{12}^{(1)}\right) \cup V\left(\bar{F}_{12}^{(2)}, \bar{F}_{21}^{(1)} \bar{F}_{12}^{(2)}\right) \\
& =V\left(\bar{F}_{21}^{(2)}, \bar{F}_{21}^{(2)}\right) \cup V\left(\bar{F}_{21}^{(2)}, \bar{F}_{12}^{(1)}\right) \cup V\left(\bar{F}_{12}^{(2)}, \bar{F}_{21}^{(1)}\right) \cup V\left(\bar{F}_{12}^{(2)}, \bar{F}_{12}^{(2)}\right)
\end{aligned}
$$


e $V\left(\bar{\zeta}_{3}^{\mathbf{X}}, \bar{\zeta}_{4}^{\mathbf{X}}\right)$ é equidimensional de dimensão $m=2$.

Suponha que para algum $m>2$ o conjunto algébrico $V_{m-1}$ é equidimensional de dimensão $m-1$. Demonstraremos que $V_{m}$ é equidimensional de dimensão $m$. Para isto, denote os geradores $\bar{\zeta}_{m}^{\mathbf{X}}, \ldots, \bar{\zeta}_{2 m-2}^{\mathbf{X}}$ de $V_{m-1}$ por $g_{m}, \ldots, g_{2 m-2}$, respectivamente, e os geradores $\bar{\zeta}_{m+1}^{\mathbf{X}}, \ldots, \bar{\zeta}_{2 m}^{\mathbf{X}}$ de $V_{m}$ por $h_{m+1}, \ldots, h_{2 m}$, respectivamente. Sejam $\mathbf{A}=\left\{\bar{F}_{21}^{(m)}\right\} \cup \mathbf{X}$ e $\mathbf{B}=\left\{\bar{F}_{12}^{(m)}\right\} \cup \mathbf{X}$, então

$$
\begin{aligned}
V\left(h_{m+1}, \ldots, h_{2 m}\right) & =V\left(h_{m+1}, \ldots, h_{2 m-1}, \bar{F}_{21}^{(m)} \bar{F}_{12}^{(m)}\right) \\
& =V\left(\bar{F}_{21}^{m}, h_{m+1}, \ldots, h_{2 m-1}\right) \cup V\left(\bar{F}_{12}^{m}, h_{m+1}, \ldots, h_{m-1}\right) \\
& =V\left(\bar{F}_{21}^{m}, h_{m+1}^{\mathbf{A}}, \ldots, h_{2 m-1}^{\mathbf{A}}\right) \cup V\left(\bar{F}_{12}^{m}, h_{m+1}^{\mathbf{B}}, \ldots, h_{2 m-1}^{\mathbf{B}}\right) .
\end{aligned}
$$

Temos que $h_{k}^{\mathbf{A}}$ é obtido de $g_{k-1}$ utilizando a mudança de variáveis dada por $\bar{F}_{21}^{(l)} \longleftrightarrow \bar{F}_{21}^{(l)}$ e $\bar{F}_{12}^{(l)} \longleftrightarrow \bar{F}_{12}^{(l-1)}$, para $k=m+1, \ldots, 2 m-1$. Além disso, $h_{k}^{\mathbf{B}}$ também é obtido de $g_{k-1}$ fazendo a mudança de variáveis dada por $\bar{F}_{21}^{(l)} \longleftrightarrow \bar{F}_{21}^{(l-1)}$ e $\bar{F}_{12}^{(l)} \longleftrightarrow \bar{F}_{12}^{(l)}$, para $k=m+1, \ldots, 2 m-1$. Logo, $V\left(\bar{F}_{21}^{m}, h_{m+1}^{\mathbf{A}}, \ldots, h_{2 m-1}^{\mathbf{A}}\right)$ e $V\left(\bar{F}_{12}^{m}, h_{m+1}^{\mathbf{B}}, \ldots, h_{m-1}^{\mathbf{B}}\right)$ são equidimensionais de dimensão $1+$ $(m-1)=m$. Consequentemente, $V_{m}=V\left(\bar{\zeta}_{2 m}^{\mathbf{X}}, \bar{\zeta}_{2 m-1}^{\mathbf{X}}, \ldots, \bar{\zeta}_{m+1}^{\mathbf{X}}\right)$ é equidimensional de dimensão $m$.

Proposição 3.2.5. $U\left(\mathfrak{g}_{m}(2)\right)$ é livre como um módulo sobre seu centro para todo $m \geq 1$.

Demonstração. Suponha $n=2$ e $m \geq 1$. Pelo Lema 1.5.24, a sequência

$$
\bar{F}_{22}^{(1)}, \bar{F}_{22}^{(2)}, \ldots, \bar{F}_{22}^{(m)}, \bar{\zeta}_{1}, \bar{\zeta}_{2}, \ldots, \bar{\zeta}_{2 m}
$$

é regular se, e somente se, $\bar{\zeta}_{1}^{\mathbf{Y}}, \ldots, \bar{\zeta}_{2 m}^{\mathbf{Y}}$ é regular, onde $\mathbf{Y}=\left\{\bar{F}_{22}^{(1)}, \bar{F}_{22}^{(2)}, \ldots, \bar{F}_{22}^{(m)}\right\}$. Como $\bar{\zeta}_{k}^{\mathbf{Y}}=\bar{F}_{11}^{(k)}$ para todo $k=1, \ldots, m$, temos que a sequência $\bar{\zeta}_{1}^{\mathbf{Y}}, \ldots, \bar{\zeta}_{2 m}^{\mathbf{Y}}$ é regular se, e somente se, $\bar{\zeta}_{m+1}^{\mathbf{X}}, \ldots, \bar{\zeta}_{2 m}^{\mathbf{X}}$, onde $\mathbf{X}=\left\{\bar{F}_{i i}^{(l)} \mid i=1,2 ; l=1, \ldots, m\right\}$. Pelo Lema 3.2.4,

$$
V\left(\bar{\zeta}_{2 m}^{\mathbf{X}}, \ldots, \bar{\zeta}_{m+1}^{\mathbf{X}}\right) \subset \mathbb{C}^{2 m}
$$

é equidimensional de dimensão $m$. Logo, pela Proposição $1.5 .20, \bar{\zeta}_{m+1}^{\mathbf{X}}, \ldots, \bar{\zeta}_{2 m}^{\mathbf{X}}$ é regular, implicando que a sequência (3.5) é regular. Como qualquer subsequência de uma sequência regular é regular, então $\bar{\zeta}_{1}, \bar{\zeta}_{2}, \ldots, \bar{\zeta}_{2 m}$ é regular. Aplicando o Teorema 1.5 .29 , temos que $U\left(\mathfrak{g}_{m}(2)\right)$ é livre como um módulo sobre seu centro.

\subsubsection{Teorema de Kostant para $U\left(\mathfrak{g}_{m}(3)\right)$}

Até agora, provamos que $U\left(\mathfrak{g}_{m}(n)\right)$ é livre como um módulo sobre seu centro, se $n \leq 2$ ou $m \leq 2$. Provar o caso geral utilizando a mesma técnica feita até agora se mostrou muito complexo para darmos continuidade, porém utilizando-a mostraremos que $U\left(\mathfrak{g}_{m}(3)\right)$ é livre sobre seu centro. Tentativas para generalizar a demonstração dada foram feitas, porém sem sucesso.

Antes de mais nada, para facilitar o processo de indução, mudaremos as variáveis. Para cada $m$, seja $\xi_{k, m}$ o polinômio em $\mathbb{k}\left[X_{i j}^{(i)}, i, j=1,2,3 ; l=0, \ldots, m-1\right]$ obtido de $\bar{\zeta}_{k}$ fazendo a mudança de variável $\bar{F}_{i j}^{(l)} \leftrightarrow X_{i j}^{(m-l)}$. Isto é,

$$
\xi_{k, m}=\sum_{\substack{i_{1}<\cdots<i_{s} \\ j_{1}+\cdots+j_{s}=k}} \sum_{\sigma \in \mathfrak{S}_{s}} \operatorname{sgn}(\sigma) X_{i_{\sigma(1)}\left(m-j_{1}\right)}^{i_{1}} \cdots X_{i_{\sigma(s)} i_{s}}^{\left(m-j_{s}\right)}
$$

Assim, $\xi_{s(m-1)+r, m}=\xi_{s(m-2)+r, m-1}$, para todo $s=1,2,3, r=1, \ldots, m$. 
Considere a sequência

$$
\left\{X_{22}^{(i)}\right\}_{i=1}^{m-1} \cup\left\{X_{33}^{(i)}\right\}_{i=1}^{m-1} \cup\left\{X_{12}^{(0)}, X_{13}^{(0)}, X_{23}^{(0)} X_{21}^{(m-1)}, X_{31}^{(m-1)}, X_{32}^{(m-1)}\right\} \cup\left\{\xi_{i, m}\right\}_{i=1}^{3 m}
$$

então $\xi_{i, m}^{\mathbf{A}}=X_{11}^{(i)}$, para $i=1, \ldots, m-1$, e $\xi_{s m, m}^{\mathbf{A}}$ é o polinômio simétrico de grau $s$ nas 3 variáveis $X_{11}^{(0)}, X_{22}^{(0)}, X_{33}^{(0)}$, para $s=1,2,3$, onde

$$
\mathbf{A}=\left\{X_{22}^{(1)}, X_{22}^{(2)}, \ldots, X_{22}^{(m-1)}, X_{33}^{(1)}, X_{33}^{(2)}, \ldots, X_{33}^{(m-1)}, X_{21}^{(0)}, X_{31}^{(0)}, X_{32}^{(0)}, X_{12}^{(m-1)}, X_{13}^{(m-1)} \cdot\right\}
$$

Sendo assim, para provar a regularidade da sequência (3.6), basta provar a regularidade da sequência

$$
\xi_{m+1, m}^{\mathbf{B}}, \xi_{m+2, m}^{\mathbf{B}}, \ldots, \xi_{2 m-1, m}^{\mathbf{B}}, \xi_{2 m+1, m}^{\mathbf{B}}, \xi_{2 m+2, m}^{\mathbf{B}}, \ldots, \xi_{3 m-1, m}^{\mathbf{B}},
$$

onde

$$
\mathbf{B}=\mathbf{A} \cup\left\{X_{11}^{(i)}\right\}_{i=1}^{m-1} \cup\left\{X_{11}^{(0)}, X_{22}^{(0)}, X_{33}^{(0)}\right\}
$$

Para provar que a sequência (3.7) é regular precisaremos de algumas definições e notações. Denote por $\sigma_{k}=-\xi_{2 m-k, m}^{\mathbf{B}}$ e $\tau_{k}=\xi_{3 m-k, m}^{\mathbf{B}}, k=1, \ldots, m-1$. Seja

$$
\lambda_{k}=\sum_{l=0}^{k-1} X_{21}^{(k-l-1)} X_{12}^{(l+1)} \text { e } \quad \eta_{k}=\sum_{l=0}^{k-1} X_{31}^{(k-l-1)} X_{13}^{(l+1)}+X_{32}^{(k-l-1)} X_{23}^{(l+1)},
$$

então $\sigma_{k}=\eta_{k}+\lambda_{k}$, para $k=1, \ldots, m-1$.

Como faremos mudanças de variáveis em $\tau_{k}, \sigma_{k}, \eta_{k}$ e $\lambda_{k}$, precisaremos determinar uma forma de fazer isto compactamente, para que a prova não seja desnecessariamente longa. Também tomaremos $p=m-1$. Para um conjunto $\mathbf{X}$ de variáveis, denote $\mathbb{C}[\mathbf{X}]$ como a álgebra polinomial nas variáveis em X. Seja

$$
\mathbf{X}_{p}=\bigcup_{i=1}^{2} \bigcup_{j=i+1}^{3}\left(\left\{X_{i j}^{(l)}\right\}_{l=1}^{p} \cup\left\{X_{j i}^{(l)}\right\}_{l=0}^{p-1}\right)
$$

então $\tau_{k}, \sigma_{k}, \eta_{k}, \lambda_{k} \in \mathbb{C}\left[\mathbf{X}_{p}\right], k=1, \ldots, p$, serão iguais mesmo quando vistos como elementos de $\mathbb{C}\left[\mathbf{X}_{p+1}\right]$. Porém, se provarmos que $V(A) \subset \mathbb{C}^{6 p}$ é equidimensional de dimensão $l$, então teremos que $V(A) \subset \mathbb{C}^{6(p+1)}$ é equidimensional de dimensão $l+6$, onde $A \subset \mathbb{C}\left[\mathbf{X}_{p}\right]$. Além disso, $\tau_{1}=$ $X_{21}^{(0)} X_{32}^{(0)} X_{13}^{(1)}$ e

$$
\tau_{k}=\sum_{j_{1}+j_{2}+j_{3}=k-1} X_{21}^{\left(j_{1}\right)} X_{32}^{\left(j_{2}\right)} X_{13}^{\left(j_{3}+1\right)}+\sum_{j_{1}+j_{2}+j_{3}=k-2} X_{31}^{\left(j_{1}\right)} X_{12}^{\left(j_{2}+1\right)} X_{23}^{\left(j_{3}+1\right)}
$$

onde $j_{1}, j_{2}, j_{3} \geq 0$ e $2 \leq k<m$.

Seja

$$
M=\left[\begin{array}{ccc}
* & X_{i_{4} j_{4}}^{\left(l_{4}\right)} & X_{i_{5} j_{5}}^{\left(l_{5}\right)} \\
X_{i_{1} j_{1}}^{\left(l_{1}\right)} & * & X_{i_{6} j_{6}}^{\left(l_{6}\right)} \\
X_{i_{2} j_{2}}^{\left(l_{2}\right)} & X_{i_{3} j_{3}}^{\left(l_{3}\right)} & *
\end{array}\right]
$$

denotaremos por $\tau_{i}(M)$ (respectivamente, $\eta_{i}(M), \lambda_{i}(M), \sigma_{i}(M)$ ) o polinômio obtido de $\tau_{i}$ (respectivamente, $\left.\eta_{i}, \lambda_{i}, \sigma_{i}\right)$ utilizando a mudança de variável

$$
\begin{gathered}
X_{21}^{(l)} \mapsto X_{i_{1} j_{1}}^{\left(l_{1}+l\right),}, X_{31}^{(l)} \mapsto X_{i_{2} j_{2}}^{\left(l_{2}+l\right)}, \quad X_{32}^{(l)} \mapsto X_{i_{3} j_{3}}^{\left(l_{3}+l\right)}, \\
X_{12}^{(l+1)} \mapsto X_{i_{4} j_{4}}^{\left(l_{4}+l\right)}, \quad X_{13}^{(l+1)} \mapsto X_{i_{5} j_{5}}^{\left(l_{5}+l\right)}, \quad X_{23}^{(l+1)} \mapsto X_{i_{6} j_{6}}^{\left(l_{6}+l\right)},
\end{gathered}
$$


$l=0,1, \ldots, p-1$. Sendo assim, se

$$
N=\left[\begin{array}{ccc}
* & X_{12}^{(1)} & X_{13}^{(1)} \\
X_{21}^{(0)} & * & X_{23}^{(1)} \\
X_{31}^{(0)} & X_{32}^{(0)} & *
\end{array}\right]
$$

então $\tau_{i}=\tau_{i}(N)$.

Exemplo 3.2.6. Se

$$
A=\left[\begin{array}{ccc}
* & X_{21}^{(1)} & X_{31}^{(1)} \\
X_{12}^{(0)} & * & X_{32}^{(1)} \\
X_{13}^{(0)} & X_{23}^{(0)} & *
\end{array}\right],
$$

então $\tau_{1}(A)=X_{31}^{(1)} X_{12}^{(0)} X_{23}^{(0)}$ e, se $2 \leq k<p-1$,

$$
\tau_{k}(A)=\sum_{j_{1}+j_{2}+j_{3}=k-2} X_{21}^{\left(j_{1}+1\right)} X_{32}^{\left(j_{2}+1\right)} X_{13}^{\left(j_{3}\right)}+\sum_{j_{1}+j_{2}+j_{3}=k-1} X_{31}^{\left(j_{1}+1\right)} X_{12}^{\left(j_{2}\right)} X_{23}^{\left(j_{3}\right)}
$$

e, se $1 \leq l<m$

$$
\eta_{l}(A)=\sum_{j_{1}+j+2=l-1} X_{31}^{\left(j_{1}+1\right)} X_{13}^{\left(j_{2}\right)}+X_{32}^{\left(j_{1}+1\right)} X_{23}^{\left(j_{2}\right)}
$$

Lema 3.2.7. Para todo $p \geq 1, X_{32}^{(0)} X_{13}^{(1)}, \eta_{1}, \ldots, \eta_{p} \in \mathbb{C}\left[X_{p}\right]$ é regular. Em particular, $\eta_{1}, \ldots, \eta_{p}$ é regular.

Demonstração. Basta mostrar que $X_{32}^{(0)}, \eta_{1}, \ldots, \eta_{p}$ e $X_{13}^{(1)}, \eta_{1}, \ldots, \eta_{p}$ são regulares. Provaremos que $X_{32}^{(0)}, \eta_{1}, \ldots, \eta_{p}$ é regular. A prova para a outra sequência é análoga. Note que

$$
X_{32}^{(0)} X_{13}^{(1)}, \eta_{1}, \ldots, \eta_{p}
$$

dependem apenas das variáveis $X_{32}^{(l)}, X_{23}^{(l+1)}, X_{31}^{(l)}, X_{13}^{(l+1)}, l=0, \ldots, p-1$, por isso iremos considerálos como elementos em $\mathbb{C}\left[\mathbf{X}_{p} \backslash\left\{X_{21}^{(l)}, X_{12}^{(l+1)} \mid l=0, \ldots, p-1\right\}\right]$.

Considere a sequência maior dada por

$$
X_{32}^{(0)}, X_{32}^{(1)}, \ldots, X_{32}^{(p-1)}, X_{23}^{(1)}, X_{23}^{(2)}, \ldots, X_{23}^{(p)}, \eta_{1}, \ldots, \eta_{p}
$$

então $\eta_{k}^{\mathbf{X}}=\sum_{i=1}^{k} X_{31}^{(k-i)} X_{13}^{(i)}, k=1, \ldots, p$, onde

$$
\mathbf{X}=\left\{X_{32}^{(0)}, X_{32}^{(1)}, \ldots, X_{32}^{(p-1)}, X_{23}^{(1)}, X_{23}^{(2)} \ldots, X_{23}^{(p)}\right\}
$$

Note que, ao fazer a mudança de variável $X_{31}^{(l)} \mapsto \bar{F}_{21}^{(p-l)}$ e $X_{13}^{(l+1)} \mapsto \bar{F}_{12}^{(p-l)}, l=0, \ldots, p-1$ em $\eta_{k}^{\mathbf{X}}$, obtemos $m$ polinômios de $\bar{U}\left(\mathfrak{g}_{m}(2)\right)$ utilizados no Lema 3.2.4, supondo $m=p$. Logo,

$$
V\left(\eta_{1}^{\mathbf{X}}, \ldots, \eta_{p}^{\mathbf{X}}\right) \subset \mathbb{C}^{2 p}
$$

é equidimensional de dimensão $p$. Pela Proposição 1.5.20, $\eta_{1}^{\mathbf{X}}, \ldots, \eta_{p}^{\mathbf{X}}$ é regular. Pelo Lema 1.5.24, a sequência (3.8) é regular também. Pela Proposição 1.5.20, qualquer subsequência de (3.8) também será regular, temos que $X_{32}^{(0)}, \eta_{1}, \ldots, \eta_{p}$ e $\eta_{1}, \ldots, \eta_{p}$ são regulares.

Exemplo 3.2.8. Se $p=3$, então $\eta_{1}=X_{31}^{(0)} X_{13}^{(1)}+X_{32}^{(0)} X_{23}^{(1)}$,

$$
\begin{gathered}
\eta_{2}=X_{31}^{(1)} X_{13}^{(1)}+X_{31}^{(0)} X_{13}^{(2)}+X_{32}^{(1)} X_{23}^{(1)}+X_{32}^{(0)} X_{23}^{(2)} \\
\eta_{3}=X_{31}^{(2)} X_{13}^{(1)}+X_{31}^{(1)} X_{13}^{(2)}+X_{31}^{(0)} X_{13}^{(3)}+X_{32}^{(2)} X_{23}^{(1)}+X_{32}^{(1)} X_{23}^{(2)}+X_{32}^{(0)} X_{23}^{(3)} .
\end{gathered}
$$


Assim, se $\mathbf{X}=\left\{X_{32}^{(0)}, X_{32}^{(1)}, X_{32}^{(2)}, X_{23}^{(1)}, X_{23}^{(2)}\right\}$, então $\eta_{1}^{\mathbf{X}}=X_{31}^{(0)} X_{13}^{(1)}, \eta_{2}^{\mathbf{X}}=X_{31}^{(1)} X_{13}^{(1)}+X_{31}^{(0)} X_{13}^{(2)}$ e

$$
\eta_{3}^{\mathbf{X}}=X_{31}^{(2)} X_{13}^{(1)}+X_{31}^{(1)} X_{13}^{(2)}+X_{31}^{(0)} X_{13}^{(3)} .
$$

Fazendo a mudança de variável mostrada na prova, temos os mesmos elementos do Lema 3.2.4, para $m=p=3$.

Lema 3.2.9. Para todo $p \geq 1, V\left(\tau_{1}, \ldots, \tau_{p}, \eta_{1}, \ldots, \eta_{p}\right) \subset \mathbb{C}^{6 p}$ é equidimensional de dimensão $4 p$. Isto é, $\tau_{1}, \ldots, \tau_{p}, \eta_{1}, \ldots, \eta_{p} \in \mathbb{C}\left[\mathbf{X}_{p}\right]$ é regular.

Demonstração. Suponha $p=1$, então

$$
\begin{aligned}
V\left(\tau_{1}, \eta_{1}\right) & =V\left(X_{21}^{(0)} X_{32}^{(0)} X_{13}^{(1)}, X_{32}^{(0)} X_{23}^{(1)}+X_{31}^{(0)} X_{13}^{(1)}\right) \\
& =V\left(X_{21}^{(0)}, X_{32}^{(0)} X_{23}^{(1)}+X_{31}^{(0)} X_{13}^{(1)}\right) \cup V\left(X_{32}^{(0)}, X_{31}^{(0)} X_{13}^{(1)}\right) \cup V\left(X_{13}^{(1)}, X_{32}^{(0)} X_{23}^{(1)}\right) \\
& =V\left(X_{21}^{(0)}, X_{32}^{(0)} X_{23}^{(1)}+X_{31}^{(0)} X_{13}^{(1)}\right) \cup V\left(X_{32}^{(0)}, X_{31}^{(0)}\right) \cup V\left(X_{32}^{(0)}, X_{13}^{(1)}\right) \cup V\left(X_{13}^{(1)}, X_{23}^{(1)}\right) .
\end{aligned}
$$

Logo, $V\left(\tau_{1}, \eta_{1}\right)$ é equidimensional de dimensão 4 e $\tau_{1}, \eta_{1}$ é regular.

Suponha $p>1$ e que provamos, por indução, que

$$
\tau_{1}, \ldots, \tau_{p-1}, \eta_{1}, \ldots, \eta_{p-1} \in \mathbb{C}\left[\mathbf{X}_{p-1}\right]
$$

é regular, portanto

$$
\tau_{1}, \ldots, \tau_{p-1}, \eta_{1}, \ldots, \eta_{p-1} \in \mathbb{C}\left[\mathbf{X}_{p}\right]
$$

é regular também. Provaremos que $\tau_{1}, \ldots, \tau_{p}, \eta_{1}, \ldots, \eta_{p}$ é regular. Temos

$$
\begin{aligned}
& V\left(\tau_{1}, \tau_{2}, \ldots, \tau_{p}, \eta_{1}, \eta_{2}, \ldots \eta_{p}\right) \\
= & V\left(X_{21}^{(0)} X_{32}^{(0)} X_{13}^{(1)}, X_{32}^{(0)} X_{23}^{(1)}+X_{31}^{(0)} X_{13}^{(1)}, \tau_{2}, \ldots, \tau_{p}, \eta_{2}, \ldots, \eta_{p}\right) \\
= & V\left(X_{21}^{(0)}, \tau_{2}, \ldots, \tau_{p}, \eta_{1}, \eta_{2}, \ldots, \eta_{p}\right) \cup V\left(X_{32}^{(0)}, X_{31}^{(0)}, \tau_{2}, \ldots, \tau_{p}, \eta_{2}, \ldots, \eta_{p}\right) \\
& \cup V\left(X_{32}^{(0)}, X_{13}^{(1)}, \tau_{2}, \ldots, \tau_{p}, \eta_{2}, \ldots, \eta_{p}\right) \cup V\left(X_{13}^{(1)}, X_{23}^{(1)}, \tau_{2}, \ldots, \tau_{p}, \eta_{2}, \ldots, \eta_{p}\right)
\end{aligned}
$$

Sejam $\mathbf{A}_{\mathbf{1}}=\left\{X_{32}^{(0)}, X_{31}^{(0)}\right\}, \mathbf{A}_{\mathbf{2}}=\left\{X_{32}^{(0)}, X_{13}^{(1)}\right\}, \mathbf{A}_{\mathbf{3}}=\left\{X_{13}^{(1)}, X_{23}^{(1)}\right\}$,

$$
M_{1}=\left[\begin{array}{ccc}
* & X_{12}^{(1)} & X_{13}^{(1)} \\
X_{21}^{(0)} & * & X_{23}^{(1)} \\
X_{31}^{(1)} & X_{32}^{(1)} & *
\end{array}\right], M_{2}=\left[\begin{array}{ccc}
* & X_{21}^{(0)} & X_{31}^{(0)} \\
X_{12}^{(1)} & * & X_{32}^{(1)} \\
X_{13}^{(2)} & X_{23}^{(1)} & *
\end{array}\right], M_{3}=\left[\begin{array}{ccc}
* & X_{12}^{(1)} & X_{13}^{(2)} \\
X_{21}^{(0)} & * & X_{23}^{(2)} \\
X_{31}^{(0)} & X_{32}^{(0)} & *
\end{array}\right]
$$

então $\tau_{i}\left(M_{j}\right)=\tau_{i+1}^{\mathbf{A}_{\mathbf{j}}}$ e $\eta_{i}\left(M_{j}\right)=\eta_{i+1}^{\mathbf{A}_{\mathbf{j}}}$, onde $i=1, \ldots, p-1$ e $j=1,2,3$. Logo,

$$
\begin{gathered}
V\left(X_{32}^{(0)}, X_{31}^{(0)}, \tau_{2}, \ldots, \tau_{p}, \eta_{2}, \ldots, \eta_{p}\right), V\left(X_{32}^{(0)}, X_{13}^{(1)}, \tau_{2}, \ldots, \tau_{p}, \eta_{2}, \ldots, \eta_{p}\right) \\
\operatorname{e~} V\left(X_{13}^{(1)}, X_{23}^{(1)}, \tau_{2}, \ldots, \tau_{p}, \eta_{2}, \ldots, \eta_{p}\right)
\end{gathered}
$$

são equidimensionais de dimensão $4(p-1)-2+6=4 p$.

Precisamos mostrar agora que $V\left(X_{21}^{(0)}, \tau_{2}, \ldots, \tau_{p}, \eta_{1}, \eta_{2}, \ldots, \eta_{p}\right)$ é equidimensional de dimensão $4 p$ também. Para isto, construiremos uma sequência de conjuntos algébricos. Seja $V_{1}=$ $V\left(X_{21}^{(0)}, \tau_{2}, \ldots, \tau_{p}, \eta_{1}, \eta_{2}, \ldots, \eta_{p}\right) \cap V\left(X_{12}^{(1)}\right)$, então

$$
V_{1}=V\left(X_{21}^{(0)}, X_{12}^{(1)}, \tau_{2}, \ldots, \tau_{p}, \eta_{1}, \eta_{2}, \ldots, \eta_{p}\right)
$$




$$
\begin{aligned}
= & V\left(X_{21}^{(0)}, X_{12}^{(1)}, X_{21}^{(1)}, \tau_{3}, \ldots, \tau_{p}, \eta_{1}, \eta_{2}, \ldots, \eta_{p}\right) \\
& \cup V\left(X_{21}^{(0)}, X_{12}^{(1)}, X_{32}^{(0)}, X_{31}^{(0)}, \tau_{3}, \ldots, \tau_{p}, \eta_{2}, \ldots, \eta_{p}\right) \\
& \cup V\left(X_{21}^{(0)}, X_{12}^{(1)}, X_{32}^{(0)}, X_{13}^{(1)}, \tau_{3}, \ldots, \tau_{p}, \eta_{2}, \ldots, \eta_{p}\right) \\
& \cup V\left(X_{21}^{(0)}, X_{12}^{(1)}, X_{13}^{(1)}, X_{23}^{(1)}, \tau_{3}, \ldots, \tau_{p}, \eta_{2}, \ldots, \eta_{p}\right) .
\end{aligned}
$$

Defina $V_{2}=V\left(X_{21}^{(0)}, X_{12}^{(1)}, X_{21}^{(1)}, \tau_{3}, \ldots, \tau_{p}, \eta_{1}, \eta_{2}, \ldots, \eta_{p}\right) \cap V\left(X_{12}^{(2)}\right)$, então também temos que

$$
\begin{aligned}
V_{2}= & V\left(X_{21}^{(0)}, X_{12}^{(1)}, X_{21}^{(1)}, X_{12}^{(2)}, X_{21}^{(2)}, \tau_{4}, \ldots, \tau_{p}, \eta_{1}, \eta_{2}, \ldots, \eta_{p}\right) \\
& \cup V\left(X_{21}^{(0)}, X_{12}^{(1)}, X_{21}^{(1)}, X_{12}^{(2)}, X_{32}^{(0)}, X_{31}^{(0)}, \tau_{4}, \ldots, \tau_{p}, \eta_{2}, \ldots, \eta_{p}\right) \\
& \cup V\left(X_{21}^{(0)}, X_{12}^{(1)}, X_{21}^{(1)}, X_{12}^{(2)}, X_{32}^{(0)}, X_{13}^{(1)}, \tau_{4}, \ldots, \tau_{p}, \eta_{2}, \ldots, \eta_{p}\right) \\
& \cup V\left(X_{21}^{(0)}, X_{12}^{(1)}, X_{21}^{(1)}, X_{12}^{(2)}, X_{13}^{(1)}, X_{23}^{(1)}, \tau_{4}, \ldots, \tau_{p}, \eta_{2}, \ldots, \eta_{p}\right) .
\end{aligned}
$$

Por recorrência, para cada $k=1, \ldots, p-2$,

$$
\begin{aligned}
V_{k}= & V\left(X_{21}^{(0)}, X_{21}^{(1)}, \ldots, X_{21}^{(k-1)}, X_{12}^{(1)}, X_{12}^{(2)}, \ldots, X_{12}^{(k)}, \tau_{k+1}, \ldots, \tau_{p}, \eta_{1}, \ldots, \eta_{p}\right) \\
= & V\left(X_{21}^{(0)}, X_{21}^{(1)}, \ldots, X_{21}^{(k)}, X_{12}^{(1)}, X_{12}^{(2)}, \ldots, X_{12}^{(k)}, \tau_{k+2}, \ldots, \tau_{p}, \eta_{1}, \eta_{2}, \ldots, \eta_{p}\right) \\
& \cup V\left(X_{21}^{(0)}, X_{21}^{(1)}, \ldots, X_{21}^{(k-1)}, X_{12}^{(1)}, X_{12}^{(2)}, \ldots, X_{12}^{(k)}, X_{32}^{(0)}, X_{31}^{(0)}, \tau_{k+2}, \ldots, \tau_{p}, \eta_{2}, \ldots, \eta_{p}\right) \\
& \cup V\left(X_{21}^{(0)}, X_{21}^{(1)}, \ldots, X_{21}^{(k-1)}, X_{12}^{(1)}, X_{12}^{(2)}, \ldots, X_{12}^{(k)}, X_{32}^{(0)}, X_{13}^{(1)}, \tau_{k+2}, \ldots, \tau_{p}, \eta_{2}, \ldots, \eta_{p}\right) \\
& \cup V\left(X_{21}^{(0)}, X_{21}^{(1)}, \ldots, X_{21}^{(k-1)}, X_{12}^{(1)}, X_{12}^{(2)}, \ldots, X_{12}^{(k)}, X_{13}^{(1)}, X_{23}^{(1)}, \tau_{k+2}, \ldots, \tau_{p}, \eta_{2}, \ldots, \eta_{p}\right)
\end{aligned}
$$

$\mathrm{e}$

$$
V_{p-1}=V\left(X_{21}^{(0)}, X_{21}^{(1)}, \ldots, X_{21}^{(p-2)}, X_{12}^{(1)}, X_{12}^{(2)}, \ldots, X_{12}^{(p-1)}, \tau_{p}, \eta_{1}, \ldots, \eta_{p}\right) .
$$

Para cada $k=1, \ldots, p-2$,

$$
\begin{gathered}
\mathbf{A}_{\mathbf{k} \mathbf{1}}=\left\{X_{21}^{(0)}, X_{21}^{(1)}, \ldots, X_{21}^{(k-1)}, X_{12}^{(1)}, X_{12}^{(2)}, \ldots, X_{12}^{(k)}, X_{32}^{(0)}, X_{31}^{(0)}\right\}, \\
\mathbf{A}_{\mathbf{k} \mathbf{2}}=\left\{X_{21}^{(0)}, X_{21}^{(1)}, \ldots, X_{21}^{(k-1)}, X_{12}^{(1)}, X_{12}^{(2)}, \ldots, X_{12}^{(k)}, X_{32}^{(0)}, X_{13}^{(1)}\right\}, \\
\mathbf{A}_{\mathbf{k} \mathbf{3}}=\left\{X_{21}^{(0)}, X_{21}^{(1)}, \ldots, X_{21}^{(k-1)}, X_{12}^{(1)}, X_{12}^{(2)}, \ldots, X_{12}^{(k)}, X_{13}^{(1)}, X_{23}^{(1)}\right\}, \\
M_{k 1}=\left[\begin{array}{ccc}
* & X_{12}^{(k+1)} & X_{13}^{(1)} \\
X_{21}^{(k)} & * & X_{23}^{(1)} \\
X_{31}^{(1)} & X_{32}^{(1)} & *
\end{array}\right], M_{k 2}=\left[\begin{array}{ccc}
* & X_{21}^{(k)} & X_{31}^{(0)} \\
X_{12}^{(k+1)} & * & X_{32}^{(1)} \\
X_{13}^{(2)} & X_{23}^{(1)} & *
\end{array}\right], M_{k 3}=\left[\begin{array}{ccc}
* & X_{12}^{(k+1)} & X_{13}^{(2)} \\
X_{21}^{(k)} & * & X_{23}^{(2)} \\
X_{31}^{(0)} & X_{32}^{(0)} & *
\end{array}\right] .
\end{gathered}
$$

Então, se $k=1, \ldots, p-2, \tau_{i}\left(M_{k j}\right)=\tau_{k+i+1}^{\mathbf{A}_{\mathbf{k j}}}$ e $\eta_{l}\left(M_{k j}\right)=\eta_{l+1}^{\mathbf{A}_{\mathbf{k j}}}$, para todo $i=1, \ldots, p-k, j=1,2,3$ e $l=1, \ldots, p-1$. Logo, para cada $k=1,2, \ldots, p-2$,

$$
\tau_{k+2}^{\mathbf{A}_{\mathbf{k j}}}, \ldots, \tau_{p}^{\mathbf{A}_{\mathbf{k j}}}, \eta_{2}^{\mathbf{A}_{\mathbf{k j}}}, \ldots, \eta_{p}^{\mathbf{A}_{\mathbf{k j}}}
$$

é uma sequência regular (pois é subsequência de uma sequência regular), para cada $j=1,2,3$, implicando que

$$
V\left(X_{21}^{(0)}, X_{21}^{(1)}, \ldots, X_{21}^{(k-1)}, X_{12}^{(1)}, X_{12}^{(2)}, \ldots, X_{12}^{(k)}, X_{32}^{(0)}, X_{31}^{(0)}, \tau_{k+2}, \ldots, \tau_{p}, \eta_{2}, \ldots, \eta_{p}\right),
$$




$$
V\left(X_{21}^{(0)}, X_{21}^{(1)}, \ldots, X_{21}^{(k-1)}, X_{12}^{(1)}, X_{12}^{(2)}, \ldots, X_{12}^{(k)}, X_{32}^{(0)}, X_{13}^{(1)}, \tau_{k+2}, \ldots, \tau_{p}, \eta_{2}, \ldots, \eta_{p}\right)
$$

e

$$
V\left(X_{21}^{(0)}, X_{21}^{(1)}, \ldots, X_{21}^{(k-1)}, X_{12}^{(1)}, X_{12}^{(2)}, \ldots, X_{12}^{(k)}, X_{13}^{(1)}, X_{23}^{(1)}, \tau_{k+2}, \ldots, \tau_{p}, \eta_{2}, \ldots, \eta_{p}\right)
$$

são equidimensionais de dimensão $4 p-k$.

Sendo assim, para mostrar que $V_{k}$ é equidimensional de dimensão $4 p-k$, basta mostrar que $V_{k+1}$ é equidimensional de dimensão $4 p-k-1$, para cada $k=1, \ldots, p-2$. Portanto, basta mostrarmos que $V_{p-1}$ é equidimensional de dimensão $3 p+1$. Temos que

$$
\begin{aligned}
V_{p-1}= & V\left(X_{21}^{(0)}, X_{21}^{(1)}, \ldots, X_{21}^{(p-2)}, X_{12}^{(1)}, X_{12}^{(2)}, \ldots, X_{12}^{(p-1)}, \tau_{p}, \eta_{1}, \eta_{2}, \ldots, \eta_{p}\right) \\
= & V\left(X_{21}^{(0)}, X_{21}^{(1)}, \ldots, X_{21}^{(p-2)}, X_{12}^{(1)}, X_{12}^{(2)}, \ldots, X_{12}^{(p-1)}, X_{21}^{(p-1)} X_{32}^{(0)} X_{13}^{(1)}, \eta_{1}, \eta_{2}, \ldots, \eta_{p}\right) \\
= & V\left(X_{21}^{(0)}, X_{21}^{(1)}, \ldots, X_{21}^{(p-2)}, X_{21}^{(p-1)}, X_{12}^{(1)}, X_{12}^{(2)}, \ldots, X_{12}^{(p-1)}, \eta_{1}, \eta_{2}, \ldots, \eta_{p}\right) \\
& \cup V\left(X_{21}^{(0)}, X_{21}^{(1)}, \ldots, X_{21}^{(p-2)}, X_{12}^{(1)}, X_{12}^{(2)}, \ldots, X_{12}^{(p-1)}, X_{32}^{(0)} X_{13}^{(1)}, \eta_{1}, \eta_{2}, \ldots, \eta_{p}\right) .
\end{aligned}
$$

Pelo Lema 3.2.7, ambas variedades são equidimensionais de dimensão $3 p+1$. Portanto, $V_{p-1}$ é equidimensional de dimensão $3 p+1$, implicando que $V_{1}$ é equidimensional de dimensão $4 p-1$, pelo argumento dado acima. Logo, $V\left(X_{21}^{(0)}, \tau_{2}, \ldots, \tau_{p}, \eta_{1}, \eta_{2}, \ldots, \eta_{p}\right)$ é equidimensional de dimensão $4 p$ e $\tau_{1}, \ldots, \tau_{p}, \eta_{1}, \ldots, \eta_{p}$ é regular.

Exemplo 3.2.10. Suponha $p=3$. Sendo assim, $\tau_{1}=X_{21}^{(0)} X_{32}^{(0)} X_{13}^{(1)}$,

$$
\begin{gathered}
\tau_{2}=X_{21}^{(0)} X_{32}^{(0)} X_{13}^{(2)}+X_{21}^{(0)} X_{32}^{(1)} X_{13}^{(1)}+X_{21}^{(1)} X_{32}^{(0)} X_{13}^{(1)}+X_{31}^{(0)} X_{12}^{(1)} X_{23}^{(1)}, \\
\tau_{3}=X_{21}^{(0)} X_{32}^{(0)} X_{13}^{(3)}+X_{21}^{(0)} X_{32}^{(1)} X_{13}^{(2)}+X_{21}^{(1)} X_{32}^{(0)} X_{13}^{(2)}+X_{21}^{(1)} X_{32}^{(1)} X_{13}^{(1)}+X_{21}^{(2)} X_{32}^{(0)} X_{13}^{(1)} \\
+X_{21}^{(0)} X_{32}^{(2)} X_{13}^{(1)}+X_{31}^{(0)} X_{12}^{(1)} X_{23}^{(2)}+X_{31}^{(0)} X_{12}^{(2)} X_{23}^{(1)}+X_{31}^{(1)} X_{12}^{(1)} X_{23}^{(1)} .
\end{gathered}
$$

Sendo assim,

$$
\begin{aligned}
V\left(\tau_{1}, \tau_{2}, \tau_{3}, \eta_{1}, \eta_{2}, \eta_{3}\right)= & V\left(X_{21}^{(0)}, \tau_{2}, \tau_{3}, \eta_{1}, \eta_{2}, \eta_{3}\right) \cup V\left(X_{32}^{(0)}, X_{31}^{(0)}, \tau_{2}, \tau_{3}, \eta_{2}, \eta_{3}\right) \\
& \cup V\left(X_{32}^{(0)}, X_{13}^{(1)}, \tau_{2}, \tau_{3}, \eta_{2}, \eta_{3}\right) \cup V\left(X_{13}^{(1)}, X_{23}^{(1)}, \tau_{2}, \tau_{3}, \eta_{2}, \eta_{3}\right)
\end{aligned}
$$

Sejam $\mathbf{A}_{\mathbf{1}}=\left\{X_{32}^{(0)}, X_{31}^{(0)}\right\}, \mathbf{A}_{\mathbf{2}}=\left\{X_{32}^{(0)}, X_{13}^{(1)}\right\}, \mathbf{A}_{\mathbf{3}}=\left\{X_{13}^{(1)}, X_{23}^{(1)}\right\}$, então

$$
\begin{gathered}
\tau_{2}^{\mathbf{A}_{1}}=X_{21}^{(0)} X_{32}^{(1)} X_{13}^{(1)}, \quad \tau_{3}^{\mathbf{A}_{1}}=X_{21}^{(0)} X_{32}^{(1)} X_{13}^{(2)}+X_{21}^{(1)} X_{32}^{(1)} X_{13}^{(1)}+X_{21}^{(0)} X_{32}^{(2)} X_{13}^{(1)}+X_{31}^{(1)} X_{12}^{(1)} X_{23}^{(1)}, \\
\eta_{2}^{\mathbf{A}_{1}}=X_{31}^{(1)} X_{13}^{(1)}+X_{32}^{(1)} X_{23}^{(1)}, \quad \eta_{3}^{\mathbf{A}_{1}}=X_{31}^{(2)} X_{13}^{(1)}+X_{31}^{(1)} X_{13}^{(2)}+X_{32}^{(2)} X_{23}^{(1)}+X_{32}^{(1)} X_{23}^{(2)}, \\
\tau_{2}^{\mathbf{A}_{2}}=X_{31}^{(0)} X_{12}^{(1)} X_{23}^{(1)}, \quad \tau_{3}^{\mathbf{A}_{2}}=X_{21}^{(0)} X_{32}^{(1)} X_{13}^{(2)}+X_{31}^{(0)} X_{12}^{(1)} X_{23}^{(2)}+X_{31}^{(0)} X_{12}^{(2)} X_{23}^{(1)}+X_{31}^{(1)} X_{12}^{(1)} X_{23}^{(1)}, \\
\eta_{2}^{\mathbf{A}_{2}}=X_{31}^{(0)} X_{13}^{(2)}+X_{32}^{(1)} X_{23}^{(1)}, \quad \eta_{3}^{\mathbf{A}_{2}}=X_{31}^{(1)} X_{13}^{(2)}+X_{31}^{(0)} X_{13}^{(3)}+X_{32}^{(2)} X_{23}^{(1)}+X_{32}^{(1)} X_{23}^{(2)}, \\
\tau_{2}^{\mathbf{A}_{3}}=X_{21}^{(0)} X_{32}^{(0)} X_{13}^{(2)}, \quad \tau_{3}^{\mathbf{A}_{3}}=X_{21}^{(0)} X_{32}^{(0)} X_{13}^{(3)}+X_{21}^{(0)} X_{32}^{(1)} X_{13}^{(2)}+X_{21}^{(1)} X_{32}^{(0)} X_{13}^{(2)}+X_{31}^{(0)} X_{12}^{(1)} X_{23}^{(2)}, \\
\eta_{2}^{\mathbf{A}_{3}}=X_{31}^{(0)} X_{13}^{(2)}+X_{32}^{(0)} X_{23}^{(2)}, \quad \eta_{3}^{\mathbf{A}_{3}}=X_{31}^{(1)} X_{13}^{(2)}+X_{31}^{(0)} X_{13}^{(3)}+X_{32}^{(1)} X_{23}^{(2)}+X_{32}^{(0)} X_{23}^{(3)} .
\end{gathered}
$$

Fazendo as mudanças de variáveis dadas na demonstração, temos que

$$
V\left(X_{32}^{(0)}, X_{31}^{(0)}, \tau_{2}, \tau_{3}, \eta_{2}, \eta_{3}\right), V\left(X_{32}^{(0)}, X_{13}^{(1)}, \tau_{2}, \tau_{3}, \eta_{2}, \eta_{3}\right)
$$




$$
\text { e } V\left(X_{13}^{(1)}, X_{23}^{(1)}, \tau_{2}, \tau_{3}, \eta_{2}, \eta_{3}\right)
$$

são equidimensionais de dimensão 12. Seguindo a notação da demonstração, seja

$$
V_{1}=V\left(X_{21}^{(0)}, \tau_{2}, \tau_{3}, \eta_{1}, \eta_{2}, \eta_{3}\right) \cap V\left(X_{12}^{(1)}\right),
$$

então

$$
\begin{aligned}
V_{1}= & V\left(X_{21}^{(0)}, X_{12}^{(1)}, X_{21}^{(1)}, \tau_{3}, \eta_{1}, \eta_{2}, \eta_{3}\right) \cup V\left(X_{21}^{(0)}, X_{12}^{(1)}, X_{32}^{(0)}, X_{31}^{(0)}, \tau_{3}, \eta_{2}, \eta_{3}\right) \\
& \cup V\left(X_{21}^{(0)}, X_{12}^{(1)}, X_{32}^{(0)}, X_{13}^{(1)}, \tau_{3}, \eta_{2}, \eta_{3}\right) \cup V\left(X_{21}^{(0)}, X_{12}^{(1)}, X_{13}^{(1)}, X_{23}^{(1)}, \tau_{3}, \eta_{2}, \eta_{3}\right) .
\end{aligned}
$$

Sejam $\mathbf{A}_{\mathbf{1 1}}=\left\{X_{21}^{(0)}, X_{12}^{(1)}, X_{32}^{(0)}, X_{31}^{(0)}\right\}, \mathbf{A}_{\mathbf{1 2}}=\left\{X_{21}^{(0)}, X_{12}^{(1)}, X_{32}^{(0)}, X_{13}^{(1)}\right\}$, $\mathbf{A}_{13}=\left\{X_{21}^{(0)}, X_{12}^{(1)}, X_{13}^{(1)}, X_{23}^{(1)}\right\}$,

$$
\begin{aligned}
& \tau_{3}^{\mathbf{A}_{11}}=X_{21}^{(1)} X_{32}^{(1)} X_{13}^{(1)}, \quad \eta_{2}^{\mathbf{A}_{11}}=X_{31}^{(1)} X_{13}^{(1)}+X_{32}^{(1)} X_{23}^{(1)}, \\
& \eta_{3}^{\mathbf{A}_{11}}=X_{31}^{(2)} X_{13}^{(1)}+X_{31}^{(1)} X_{13}^{(2)}+X_{32}^{(2)} X_{23}^{(1)}+X_{32}^{(1)} X_{23}^{(2)}, \\
& \tau_{3}^{\mathbf{A}_{12}}=X_{31}^{(0)} X_{12}^{(2)} X_{23}^{(1)}, \quad \eta_{2}^{\mathbf{A}_{12}}=X_{31}^{(0)} X_{13}^{(2)}+X_{32}^{(1)} X_{23}^{(1)}, \\
& \eta_{3}^{\mathbf{A}_{12}}=X_{31}^{(1)} X_{13}^{(2)}+X_{31}^{(0)} X_{13}^{(3)}+X_{32}^{(2)} X_{23}^{(1)}+X_{32}^{(1)} X_{23}^{(2)}, \\
& \tau_{3}^{\mathbf{A}_{13}}=X_{21}^{(1)} X_{32}^{(0)} X_{13}^{(2)}, \quad \eta_{2}^{\mathbf{A}_{13}}=X_{31}^{(0)} X_{13}^{(2)}+X_{32}^{(0)} X_{23}^{(2)}, \\
& \eta_{3}^{\mathbf{A}_{13}}=X_{31}^{(1)} X_{13}^{(2)}+X_{31}^{(0)} X_{13}^{(3)}+X_{32}^{(1)} X_{23}^{(2)}+X_{32}^{(0)} X_{23}^{(3)} .
\end{aligned}
$$

Portanto, temos que $\tau_{3}^{\mathbf{A}_{\mathbf{1 j}}}, \eta_{2}^{\mathbf{A}_{\mathbf{1 j}}}, \eta_{3}^{\mathbf{A}_{\mathbf{1 j}}}$ é regular, para cada $j=1,2,3$. Pelo Lema 3.2.7,

$$
\begin{aligned}
V_{2} & =V\left(X_{21}^{(0)}, X_{12}^{(1)}, X_{21}^{(1)}, \tau_{3}, \eta_{1}, \eta_{2}, \eta_{3}\right) \cap V\left(X_{12}^{(2)}\right) \\
& =V\left(X_{21}^{(0)}, X_{12}^{(1)}, X_{21}^{(1)}, X_{12}^{(2)}, X_{21}^{(2)} X_{32}^{(0)} X_{13}^{(1)}, \eta_{1}, \eta_{2}, \eta_{3}\right) \\
& =V\left(X_{21}^{(0)}, X_{12}^{(1)}, X_{21}^{(1)}, X_{12}^{(2)}, X_{21}^{(2)}, \eta_{1}, \eta_{2}, \eta_{3}\right) \cup V\left(X_{21}^{(0)}, X_{12}^{(1)}, X_{21}^{(1)}, X_{12}^{(2)}, X_{32}^{(0)} X_{13}^{(1)}, \eta_{1}, \eta_{2}, \eta_{3}\right)
\end{aligned}
$$

é equidimensional de dimensão 10, implicando que $V_{1}$ é equidimensional de dimensão 11.

Proposição 3.2.11. Seja $p \geq 1$. Para todo $0 \leq q \leq p, \tau_{1}, \ldots, \tau_{p}, \eta_{1}, \ldots, \eta_{p-q}, \eta_{p-q+1}+\lambda_{1}, \ldots, \eta_{p}+$ $\lambda_{q}$ é regular.

Demonstração. Suponha $p=1$. Se $q=0$, então, pelo Lema 3.2.9, $\tau_{1}, \eta_{1}$ é regular. Se $q=1$, então a decomposição de $V\left(\tau_{1}, \eta_{1}+\lambda_{1}\right)$ em variedades irredutíveis é

$$
\begin{aligned}
V\left(\tau_{1}, \eta_{1}+\lambda_{1}\right)= & V\left(X_{21}^{(0)} X_{32}^{(0)} X_{13}^{(1)}, X_{21}^{(0)} X_{12}^{(1)}+X_{32}^{(0)} X_{13}^{(1)}+X_{32}^{(0)} X_{23}^{(1)}\right) \\
= & V\left(X_{21}^{(0)}, X_{32}^{(0)} X_{13}^{(1)}+X_{32}^{(0)} X_{23}^{(1)}\right) \\
& \cup V\left(X_{32}^{(0)}, X_{21}^{(0)} X_{12}^{(1)}+X_{32}^{(0)} X_{23}^{(1)}\right) \cup V\left(X_{13}^{(1)}, X_{21}^{(0)} X_{12}^{(1)}+X_{32}^{(0)} X_{13}^{(1)}\right) .
\end{aligned}
$$

Logo, $V\left(\tau_{1}, \eta_{1}+\lambda_{1}\right)$ é equidimensional de dimensão 2 e $\tau_{1}, \eta_{1}+\lambda_{1}$ é regular.

Suponha $p>1$. A hipótese de indução em $p$ é que

$$
\tau_{1}, \ldots, \tau_{p-1}, \eta_{1}, \ldots, \eta_{p-1-q}, \eta_{p-q}+\lambda_{1}, \ldots, \eta_{p-1}+\lambda_{q}
$$

é regular, para todo $0 \leq q \leq p-1$. Dividiremos a demonstração de que

$$
\tau_{1}, \ldots, \tau_{p}, \eta_{1}, \ldots, \eta_{p-q}, \eta_{p-q+1}+\lambda_{1}, \ldots, \eta_{p}+\lambda_{q}
$$


é regular, para todo $0 \leq q \leq p$, em duas partes. Na primeira, provaremos por indução em $0 \leq q \leq$ $p-1$ que a afirmação é verdade. Na segunda parte, utilizaremos a primeira parte e a hipótese de indução para provar que a afirmação é verdade para $q=p$.

Demonstraremos, por indução em $0 \leq q<p$ que

$$
\tau_{1}, \ldots, \tau_{p}, \eta_{1}, \ldots, \eta_{p-q}, \eta_{p-q+1}+\lambda_{1}, \ldots, \eta_{p}+\lambda_{q}
$$

é regular. Se $q=0$, então, pelo Lema 3.2.9, temos que a afirmação é verdadeira. Suponha $0<q<p$. Por indução,

$$
\tau_{1}, \ldots, \tau_{p}, \eta_{1}, \ldots, \eta_{p-l}, \eta_{p-l+1}+\lambda_{1}, \ldots, \eta_{p}+\lambda_{l}
$$

é regular, para todo $0 \leq l<q$. Como $p-q \geq 1$, temos que

$$
\begin{aligned}
& V\left(\tau_{1}, \tau_{2}, \ldots, \tau_{p}, \eta_{1}, \eta_{2} \ldots, \eta_{p-q}, \eta_{p-q+1}+\lambda_{1}, \eta_{p-q+2}+\lambda_{2}, \ldots, \eta_{p}+\lambda_{q}\right) \\
= & V\left(X_{21}^{(0)}, \tau_{2}, \ldots, \tau_{p}, \eta_{2}, \ldots, \eta_{p-q+1}+\lambda_{1}, \ldots, \eta_{p}+\lambda_{q}\right) \\
& \cup V\left(X_{32}^{(0)}, X_{31}^{(0)}, \tau_{2}, \ldots, \tau_{p}, \eta_{2}, \ldots, \eta_{p-q+1}+\lambda_{1}, \ldots, \eta_{p}+\lambda_{q}\right) \\
& \cup V\left(X_{32}^{(0)}, X_{13}^{(1)}, \tau_{2}, \ldots, \tau_{p}, \eta_{2}, \ldots, \eta_{p-q+1}+\lambda_{1}, \ldots, \eta_{p}+\lambda_{q}\right) \\
& \cup V\left(X_{13}^{(1)}, X_{23}^{(1)}, \tau_{2}, \ldots, \tau_{p}, \eta_{2}, \ldots, \eta_{p-q+1}+\lambda_{1}, \ldots, \eta_{p}+\lambda_{q}\right) .
\end{aligned}
$$

Sejam $\mathbf{A}_{\mathbf{1}}=\left\{X_{32}^{(0)}, X_{31}^{(0)}\right\}, \mathbf{A}_{\mathbf{2}}=\left\{X_{32}^{(0)}, X_{13}^{(1)}\right\}, \mathbf{A}_{\mathbf{3}}=\left\{X_{13}^{(1)}, X_{23}^{(1)}\right\}$

$$
M_{1}=\left[\begin{array}{ccc}
* & X_{12}^{(1)} & X_{13}^{(1)} \\
X_{21}^{(0)} & * & X_{23}^{(1)} \\
X_{31}^{(1)} & X_{32}^{(1)} & *
\end{array}\right], M_{2}=\left[\begin{array}{ccc}
* & X_{21}^{(0)} & X_{31}^{(0)} \\
X_{12}^{(1)} & * & X_{32}^{(1)} \\
X_{13}^{(2)} & X_{23}^{(1)} & *
\end{array}\right], M_{3}=\left[\begin{array}{ccc}
* & X_{12}^{(1)} & X_{13}^{(2)} \\
X_{21}^{(0)} & * & X_{23}^{(2)} \\
X_{31}^{(0)} & X_{32}^{(0)} & *
\end{array}\right]
$$

então, para cada $j=1,2,3, \tau_{i}\left(M_{j}\right)=\tau_{i+1}^{\mathbf{A}_{\mathbf{j}}}, \eta_{a}\left(M_{j}\right)=\eta_{a+1}^{\mathbf{A}_{\mathbf{j}}}, \eta_{p-q+b-1}\left(M_{j}\right)+\lambda_{b}\left(M_{j}\right)=\eta_{p-q+b}^{\mathbf{A}_{\mathbf{j}}}+\lambda_{b}^{\mathbf{A}_{\mathbf{j}}}$, para $i=1, \ldots, m-1, a=1, \ldots, p-q-1, b=1, \ldots, q$. Pela hipótese de indução em $p$ e como $q \leq p-1$,

$$
\tau_{2}^{\mathbf{A}_{\mathbf{j}}}, \ldots, \tau_{p}^{\mathbf{A}_{\mathbf{j}}}, \eta_{2}^{\mathbf{A}_{\mathbf{j}}}, \ldots, \eta_{p-q}^{\mathbf{A}_{\mathbf{j}}}, \eta_{p-q+1}^{\mathbf{A}_{\mathbf{j}}}+\lambda_{1}^{\mathbf{A}_{\mathbf{j}}}, \ldots, \eta_{p}^{\mathbf{A}_{\mathbf{j}}}+\lambda_{q}^{\mathbf{A}_{\mathbf{j}}}
$$

é regular, pois a mudança de variável acima determina uma correspondência entre essa sequência e

$$
\tau_{1}, \tau_{2}, \ldots, \tau_{p-1}, \eta_{1}, \eta_{2}, \ldots, \eta_{p-1-q}, \eta_{p-q}+\lambda_{1}, \ldots, \eta_{p-1}+\lambda_{q} .
$$

Pelo Lema 1.5.24 e Proposição 1.5.20,

$$
\begin{aligned}
& V\left(X_{32}^{(0)}, X_{31}^{(0)}, \tau_{2}, \ldots, \tau_{p}, \eta_{2}, \ldots, \eta_{p-q+1}+\lambda_{1}, \ldots, \eta_{p}+\lambda_{q}\right), \\
& V\left(X_{32}^{(0)}, X_{13}^{(1)}, \tau_{2}, \ldots, \tau_{p}, \eta_{2}, \ldots, \eta_{p-q+1}+\lambda_{1}, \ldots, \eta_{p}+\lambda_{q}\right), \\
& V\left(X_{13}^{(1)}, X_{23}^{(1)}, \tau_{2}, \ldots, \tau_{p}, \eta_{2}, \ldots, \eta_{p-q+1}+\lambda_{1}, \ldots, \eta_{p}+\lambda_{q}\right)
\end{aligned}
$$

são equidimensionais de dimensão $4 p$.

Temos ainda que, se $\mathbf{C}=\left\{X_{21}^{(0)}, X_{12}^{(1)}\right\}$ e

$$
M=\left[\begin{array}{ccc}
* & X_{12}^{(2)} & X_{13}^{(1)} \\
X_{21}^{(1)} & * & X_{23}^{(1)} \\
X_{31}^{(0)} & X_{32}^{(0)} & *
\end{array}\right]
$$


então $\tau_{i}(M)=\tau_{i+1}^{\mathbf{C}}, \eta_{a}(M)=\eta_{a}^{\mathbf{C}}=\eta_{a}$,

$$
\eta_{p-q+1}^{\mathbf{C}}+\lambda_{1}^{\mathbf{C}}=\eta_{p-q+1}^{\mathbf{C}}=\eta_{p-q+1}
$$

$\eta_{p-q+2}^{\mathbf{C}}+\lambda_{2}^{\mathbf{C}}=\eta_{p-q+2}$ e $\eta_{p-q+2+b}^{\mathbf{C}}+\lambda_{b+2}^{\mathbf{C}}=\eta_{p-q+2+b}+\lambda_{b}(M)$, onde $i=1, \ldots, p-1, a=1, \ldots, p-q$, $b=1, \ldots, q-2$. Logo,

$$
\begin{aligned}
& V\left(X_{21}^{(0)}, \tau_{2}, \ldots, \tau_{p}, \eta_{2}, \ldots, \eta_{p-q+1}+\lambda_{1}, \ldots, \eta_{p}+\lambda_{q}\right) \cap V\left(X_{12}^{(1)}\right) \\
= & V\left(X_{21}^{(0)}, X_{12}^{(1)}, \tau_{2}^{\mathbf{A}}, \ldots, \tau_{p}^{\mathbf{A}}, \eta_{2}^{\mathbf{A}}, \ldots, \eta_{p-q+1}^{\mathbf{A}}+\lambda_{1}^{\mathbf{A}}, \ldots, \eta_{p}^{\mathbf{A}}+\lambda_{q}^{\mathbf{A}}\right) \\
= & V\left(X_{21}^{(0)}, X_{12}^{(1)}, \tau_{1}(M), \ldots, \tau_{p-1}(M), \eta_{1}, \eta_{2}, \ldots, \eta_{p-q+2}, \eta_{p-q+3}+\lambda_{1}(M), \ldots, \eta_{p}+\lambda_{q-2}(M)\right)
\end{aligned}
$$

é equidimensional de dimensão $4 p-1$, pela hipótese de indução em $q$. Portanto,

$$
V\left(X_{21}^{(0)}, \tau_{2}, \ldots, \tau_{p}, \eta_{2}, \ldots, \eta_{p-q+1}+\lambda_{1}, \ldots, \eta_{p}+\lambda_{q}\right)
$$

também é equidimensional de dimensão $4 p$ e

$$
\tau_{1}, \tau_{2}, \ldots, \tau_{p}, \eta_{1}, \eta_{2} \ldots, \eta_{p-q}, \eta_{p-q+1}+\lambda_{1}, \eta_{p-q+2}+\lambda_{2}, \ldots, \eta_{p}+\lambda_{q}
$$

é regular, se $0 \leq q \leq p-1$.

Sendo assim, suponha $q=p$. Isto é, queremos mostrar a regularidade da sequência

$$
\tau_{1}, \ldots, \tau_{p}, \eta_{1}+\lambda_{1}, \eta_{2}+\lambda_{2}, \ldots, \eta_{p}+\lambda_{p}
$$

Como $\tau_{1}=X_{21}^{(0)} X_{32}^{(0)} X_{13}^{(1)}$, temos que

$$
\begin{aligned}
& V\left(\tau_{1}, \ldots, \tau_{p}, \eta_{1}+\lambda_{1}, \eta_{2}+\lambda_{2}, \ldots, \eta_{p}+\lambda_{p}\right) \\
= & V\left(X_{21}^{(0)}, \tau_{2}, \tau_{3}, \ldots, \tau_{p}, \eta_{1}+\lambda_{1}, \eta_{2}+\lambda_{2}, \ldots, \eta_{p}+\lambda_{p}\right) \\
& \cup V\left(X_{32}^{(0)} \tau_{2}, \tau_{3}, \ldots, \tau_{p}, \eta_{1}+\lambda_{1}, \eta_{2}+\lambda_{2}, \ldots, \eta_{p}+\lambda_{p}\right) \\
& \cup V\left(X_{13}^{(1)}, \tau_{2}, \tau_{3}, \ldots, \tau_{p}, \eta_{1}+\lambda_{1}, \eta_{2}+\lambda_{2}, \ldots, \eta_{p}+\lambda_{p}\right)
\end{aligned}
$$

Sejam $\mathbf{X}=\left\{X_{21}^{(0)}\right\}, \mathbf{Y}=\left\{X_{32}^{(0)}\right\}, \mathbf{Z}=\left\{X_{13}^{(1)}\right\}$. Lembre que denotamos $\sigma_{i}=\eta_{i}+\lambda_{i}$, para cada $i=1, \ldots, p$. Temos que $\tau_{i}^{\mathbf{X}}$ e $\sigma_{i}^{\mathbf{X}}$ são obtidos de $\tau_{i}^{\mathbf{Y}}$ e $\sigma_{i}^{\mathbf{Y}}$, respectivamente, fazendo a mudança de variável

$$
X_{i j}^{(l)} \mapsto \begin{cases}X_{12}^{(l)}, & \text { se }(i, j)=(2,3) ; \\ X_{21}^{(l)}, & \text { se }(i, j)=(3,2) ; \\ X_{13}^{(l)}, & \text { se }(i, j)=(1,3) ; \\ X_{31}^{(l)}, & \text { se }(i, j)=(3,1) ; \\ X_{23}^{(l)}, & \text { se }(i, j)=(1,2) ; \\ X_{32}^{(l)}, & \text { se }(i, j)=(2,1) .\end{cases}
$$


Além disso, $\tau_{i}^{\mathbf{X}}$ e $\sigma_{i}^{\mathbf{X}}$ são obtidos de $\tau_{i}^{\mathbf{Z}}$ e $\sigma_{i}^{\mathbf{Z}}$, respectivamente, fazendo a mudança de variável

$$
X_{i j}^{(l)} \mapsto \begin{cases}X_{12}^{(l+1)}, & \text { se }(i, j)=(3,1) ; \\ X_{21}^{(l-1)}, & \text { se }(i, j)=(1,3) ; \\ X_{13}^{(l+1)}, & \text { se }(i, j)=(2,1) ; \\ X_{31}^{(l-1)}, & \text { se }(i, j)=(1,2) ; \\ X_{23}^{(l)}, & \text { se }(i, j)=(2,3) ; \\ X_{32}^{(l)}, & \text { se }(i, j)=(3,2) .\end{cases}
$$

Portanto, basta mostrar que

$$
V\left(X_{21}^{(0)}, \tau_{2}, \tau_{3}, \ldots, \tau_{p}, \eta_{1}+\lambda_{1}, \eta_{2}+\lambda_{2}, \ldots, \eta_{p}+\lambda_{p}\right)
$$

é equidimensional de dimensão $4 p$. Seja $\mathbf{D}=\mathbf{X} \cup\left\{X_{12}^{(1)}\right\}$ e

$$
N=\left[\begin{array}{ccc}
* & X_{12}^{(2)} & X_{13}^{(1)} \\
X_{21}^{(1)} & * & X_{23}^{(1)} \\
X_{31}^{(0)} & X_{32}^{(0)} & *
\end{array}\right],
$$

então $\tau_{i}(N)=\tau_{i+1}^{\mathbf{D}}, \sigma_{1}^{\mathbf{D}}=\eta_{1}, \sigma_{2}^{\mathbf{D}}=\eta_{2}, \sigma_{2+j}^{\mathbf{D}}=\eta_{2+j}+\lambda_{i}(N)$, para todo $i=1, \ldots, p-1 \mathrm{e}$ $j=1, \ldots, p-2$. Como já provamos que

$$
\tau_{1}, \ldots, \tau_{p}, \eta_{1}, \eta_{2}, \eta_{3}+\lambda_{1}, \eta_{4}+\lambda_{2}, \ldots, \eta_{p}+\lambda_{p-2}
$$

é regular e toda subsequência desta sequência será regular, temos que

$$
\begin{aligned}
& V\left(X_{21}^{(0)}, \tau_{2}, \tau_{3}, \ldots, \tau_{p}, \eta_{1}+\lambda_{1}, \eta_{2}+\lambda_{2}, \ldots, \eta_{p}+\lambda_{p}\right) \cap V\left(X_{12}^{(1)}\right) \\
= & V\left(X_{21}^{(0)}, X_{12}^{(1)}, \tau_{1}(N), \tau_{2}(N), \ldots, \tau_{p-1}(N), \eta_{1}, \eta_{2}, \eta_{3}+\lambda_{1}(N), \ldots, \eta_{p}+\lambda_{p-2}(N)\right)
\end{aligned}
$$

é equidimensional de dimensão $4 p-1$. Logo,

$$
V\left(X_{21}^{(0)}, \tau_{2}, \tau_{3}, \ldots, \tau_{p}, \eta_{1}+\lambda_{1}, \eta_{2}+\lambda_{2}, \ldots, \eta_{p}+\lambda_{p}\right)
$$

é equidimensional de dimensão $4 p$. Implicando que

$$
\tau_{1}, \ldots, \tau_{p}, \eta_{1}+\lambda_{1}, \eta_{2}+\lambda_{2}, \ldots, \eta_{p}+\lambda_{p}
$$

é regular.

Exemplo 3.2.12. Se $p=3$, então $\sigma_{1}=X_{31}^{(0)} X_{13}^{(1)}+X_{32}^{(0)} X_{23}^{(1)}+X_{21}^{(0)} X_{12}^{(1)}$,

$$
\begin{aligned}
\sigma_{2}= & X_{31}^{(1)} X_{13}^{(1)}+X_{31}^{(0)} X_{13}^{(2)}+X_{32}^{(1)} X_{23}^{(1)}+X_{32}^{(0)} X_{23}^{(2)}+X_{21}^{(1)} X_{12}^{(1)}+X_{21}^{(0)} X_{12}^{(2)} \\
\sigma_{3}= & X_{31}^{(2)} X_{13}^{(1)}+X_{31}^{(1)} X_{13}^{(2)}+X_{31}^{(0)} X_{13}^{(3)}+X_{32}^{(2)} X_{23}^{(1)}+X_{32}^{(1)} X_{23}^{(2)}+X_{32}^{(0)} X_{23}^{(3)} \\
& +X_{21}^{(0)} X_{12}^{(3)}+X_{21}^{(1)} X_{12}^{(2)}+X_{21}^{(2)} X_{12}^{(1)} .
\end{aligned}
$$

Não exemplificarei todos os casos da indução feita na demonstração da Proposição 3.2.11. Escreverei os elementos obtidos para o caso $q=p$ para que o leitor possa ter estes exemplos explícitos caso 
queira conferir a prova. Como feito na demonstração da Proposição 3.2.11,

$$
\begin{aligned}
V\left(\tau_{1}, \tau_{2}, \tau_{3}, \sigma_{1}, \sigma_{2}, \sigma_{3}\right)= & V\left(X_{21}^{(0)} X_{32}^{(0)} X_{13}^{(1)}, \tau_{2}, \tau_{3}, \sigma_{1}, \sigma_{2}, \sigma_{3}\right) \\
= & V\left(X_{21}^{(0)}, \tau_{2}, \tau_{3}, \sigma_{1}, \sigma_{2}, \sigma_{3}\right) \cup V\left(X_{32}^{(0)}, \tau_{2}, \tau_{3}, \sigma_{1}, \sigma_{2}, \sigma_{3}\right) \\
& \cup V\left(X_{13}^{(1)}, \tau_{2}, \tau_{3}, \sigma_{1}, \sigma_{2}, \sigma_{3}\right) .
\end{aligned}
$$

Sejam $\mathbf{X}=\left\{X_{21}^{(0)}\right\}, \mathbf{Y}=\left\{X_{32}^{(0)}\right\}, \mathbf{Z}=\left\{X_{13}^{(1)}\right\}$, portanto

$$
\begin{aligned}
& \tau_{2}^{\mathbf{X}}=X_{21}^{(1)} X_{32}^{(0)} X_{13}^{(1)}+X_{31}^{(0)} X_{12}^{(1)} X_{23}^{(1)}, \\
& \tau_{3}^{\mathbf{X}}=X_{21}^{(1)} X_{32}^{(0)} X_{13}^{(2)}+X_{21}^{(1)} X_{32}^{(1)} X_{13}^{(1)}+X_{21}^{(2)} X_{32}^{(0)} X_{13}^{(1)} \\
& +X_{31}^{(0)} X_{12}^{(1)} X_{23}^{(2)}+X_{31}^{(0)} X_{12}^{(2)} X_{23}^{(1)}+X_{31}^{(1)} X_{12}^{(1)} X_{23}^{(1)} \text {, } \\
& \sigma_{1}^{\mathbf{X}}=X_{31}^{(0)} X_{13}^{(1)}+X_{32}^{(0)} X_{23}^{(1)}=\eta_{1}, \\
& \sigma_{2}^{\mathbf{X}}=X_{31}^{(1)} X_{13}^{(1)}+X_{31}^{(0)} X_{13}^{(2)}+X_{32}^{(1)} X_{23}^{(1)}+X_{32}^{(0)} X_{23}^{(2)}+X_{21}^{(1)} X_{12}^{(1)}=\eta_{2}+X_{21}^{(1)} X_{12}^{(1)} \text {, } \\
& \sigma_{3}^{\mathbf{X}}=X_{31}^{(2)} X_{13}^{(1)}+X_{31}^{(1)} X_{13}^{(2)}+X_{31}^{(0)} X_{13}^{(3)}+X_{32}^{(2)} X_{23}^{(1)}+X_{32}^{(1)} X_{23}^{(2)}+X_{32}^{(0)} X_{23}^{(3)} \\
& +X_{21}^{(1)} X_{12}^{(2)}+X_{21}^{(2)} X_{12}^{(1)}=\eta_{3}+X_{21}^{(1)} X_{12}^{(2)}+X_{21}^{(2)} X_{12}^{(1)}, \\
& \tau_{2}^{\mathbf{Y}}=X_{21}^{(0)} X_{32}^{(1)} X_{13}^{(1)}+X_{31}^{(0)} X_{12}^{(1)} X_{23}^{(1)}, \\
& \tau_{3}^{\mathbf{Y}}=X_{21}^{(0)} X_{32}^{(1)} X_{13}^{(2)}+X_{21}^{(1)} X_{32}^{(1)} X_{13}^{(1)}+X_{21}^{(0)} X_{32}^{(2)} X_{13}^{(1)} \\
& ++X_{31}^{(0)} X_{12}^{(1)} X_{23}^{(2)}+X_{31}^{(0)} X_{12}^{(2)} X_{23}^{(1)}+X_{31}^{(1)} X_{12}^{(1)} X_{23}^{(1)} \text {, } \\
& \sigma_{1}^{\mathbf{Y}}=X_{31}^{(0)} X_{13}^{(1)}+X_{21}^{(0)} X_{12}^{(1)}, \\
& \sigma_{2}^{\mathbf{Y}}=X_{31}^{(1)} X_{13}^{(1)}+X_{31}^{(0)} X_{13}^{(2)}+X_{32}^{(1)} X_{23}^{(1)}+X_{21}^{(1)} X_{12}^{(1)}+X_{21}^{(0)} X_{12}^{(2)} \text {, } \\
& \sigma_{3}^{\mathbf{Y}}=X_{31}^{(2)} X_{13}^{(1)}+X_{31}^{(1)} X_{13}^{(2)}+X_{31}^{(0)} X_{13}^{(3)}+X_{32}^{(2)} X_{23}^{(1)}+X_{32}^{(1)} X_{23}^{(2)} \\
& +X_{21}^{(0)} X_{12}^{(3)}+X_{21}^{(1)} X_{12}^{(2)}+X_{21}^{(2)} X_{12}^{(1)} \text {, } \\
& \tau_{2}^{\mathbf{Z}}=X_{21}^{(0)} X_{32}^{(0)} X_{13}^{(2)}+X_{31}^{(0)} X_{12}^{(1)} X_{23}^{(1)}, \\
& \tau_{3}^{\mathbf{Z}}=X_{21}^{(0)} X_{32}^{(0)} X_{13}^{(3)}+X_{21}^{(0)} X_{32}^{(1)} X_{13}^{(2)}+X_{21}^{(1)} X_{32}^{(0)} X_{13}^{(2)} \\
& +X_{31}^{(0)} X_{12}^{(1)} X_{23}^{(2)}+X_{31}^{(0)} X_{12}^{(2)} X_{23}^{(1)}+X_{31}^{(1)} X_{12}^{(1)} X_{23}^{(1)} \text {, } \\
& \sigma_{1}^{\mathbf{Z}}=X_{32}^{(0)} X_{23}^{(1)}+X_{21}^{(0)} X_{12}^{(1)} \\
& \sigma_{2}^{\mathbf{Z}}=X_{31}^{(0)} X_{13}^{(2)}+X_{32}^{(1)} X_{23}^{(1)}+X_{32}^{(0)} X_{23}^{(2)}+X_{21}^{(1)} X_{12}^{(1)}+X_{21}^{(0)} X_{12}^{(2)} \text {, } \\
& \sigma_{3}^{\mathbf{Z}}=X_{31}^{(1)} X_{13}^{(2)}+X_{31}^{(0)} X_{13}^{(3)}+X_{32}^{(2)} X_{23}^{(1)}+X_{32}^{(1)} X_{23}^{(2)}+X_{32}^{(0)} X_{23}^{(3)} \\
& +X_{21}^{(0)} X_{12}^{(3)}+X_{21}^{(1)} X_{12}^{(2)}+X_{21}^{(2)} X_{12}^{(1)} \text {. }
\end{aligned}
$$

Sendo assim, $\tau_{i}^{\mathbf{X}}$ e $\sigma_{i}^{\mathbf{X}}$ podem ser obtidos de $\tau_{i}^{\mathbf{Y}}$ e $\sigma_{i}^{\mathbf{Y}}\left(\tau_{i}^{\mathbf{Z}}\right.$ e $\left.\sigma_{i}^{\mathbf{Z}}\right)$ utilizando uma mudança de variável, 
respectivamente, portanto basta mostrar que $\tau_{2}^{\mathbf{X}}, \tau_{3}^{\mathbf{X}}, \sigma_{1}^{\mathbf{X}}, \sigma_{2}^{\mathbf{X}}, \sigma_{3}^{\mathbf{X}}$ é regular. Sejam $\mathbf{D}=\mathbf{X} \cup\left\{X_{12}^{(1)}\right\}$ e

$$
N=\left[\begin{array}{ccc}
* & X_{12}^{(2)} & X_{13}^{(1)} \\
X_{21}^{(1)} & * & X_{23}^{(1)} \\
X_{31}^{(0)} & X_{32}^{(0)} & *
\end{array}\right],
$$

então $\tau_{2}^{\mathbf{D}}=X_{21}^{(1)} X_{32}^{(0)} X_{13}^{(1)}=\tau_{1}(N)$,

$$
\begin{gathered}
\tau_{3}^{\mathbf{D}}=X_{21}^{(1)} X_{32}^{(0)} X_{13}^{(2)}+X_{21}^{(1)} X_{32}^{(1)} X_{13}^{(1)}+X_{21}^{(2)} X_{32}^{(0)} X_{13}^{(1)}+X_{31}^{(0)} X_{12}^{(2)} X_{23}^{(1)}=\tau_{2}(N), \\
\sigma_{1}^{\mathbf{D}}=X_{31}^{(0)} X_{13}^{(1)}+X_{32}^{(0)} X_{23}^{(1)}=\eta_{1}, \\
\sigma_{2}^{\mathbf{D}}=X_{31}^{(1)} X_{13}^{(1)}+X_{31}^{(0)} X_{13}^{(2)}+X_{32}^{(1)} X_{23}^{(1)}+X_{32}^{(0)} X_{23}^{(2)}=\eta_{2}, \\
\sigma_{3}^{\mathbf{D}}=X_{31}^{(2)} X_{13}^{(1)}+X_{31}^{(1)} X_{13}^{(2)}+X_{31}^{(0)} X_{13}^{(3)}+X_{32}^{(2)} X_{23}^{(1)}+X_{32}^{(1)} X_{23}^{(2)}+X_{32}^{(0)} X_{23}^{(3)}+X_{21}^{(1)} X_{12}^{(2)} \\
=\eta_{3}+X_{21}^{(1)} X_{12}^{(2)}=\eta_{3}+\lambda_{1}(N) .
\end{gathered}
$$

Corolário 3.2.13. Para todo $m>1, \bar{\zeta}_{1}, \bar{\zeta}_{2}, \ldots, \bar{\zeta}_{3 m}$ é regular.

Demonstração. Pela Proposição 3.2.11, supondo $p=m+1$, a sequência (3.7) é regular. Portanto, a sequência (3.6) é regular também. Pela Proposição 1.5.20, qualquer subsequência de (3.6) será regular também. Em particular, $\xi_{1, m}, \ldots, \xi_{3 m, m}$ é regular. Desfazendo a mudança de variável que fizemos em $\bar{\zeta}_{1}, \bar{\zeta}_{2}, \ldots, \bar{\zeta}_{3 m}$ para obter $\xi_{1, m}, \xi_{2, m}, \ldots, \xi_{3 m, m}$, temos que $\bar{\zeta}_{1}, \bar{\zeta}_{2}, \ldots, \bar{\zeta}_{3 m}$ é regular.

Aplicando o Teorema 1.5.29 no Corolário 3.2.13, temos a seguinte versão do Teorema de Kostant para $U\left(\mathfrak{g}_{m}(3)\right)$.

Teorema 3.2.14. $U\left(\mathfrak{g}_{m}(3)\right)$ é livre como um módulo sobre seu centro, para todo $m \geq 1$.

\subsection{Subálgebra de Gelfand-Tsetlin}

Consideraremos outra subálgebra de $U\left(\mathfrak{g}_{m}(n)\right)$. Para cada $p \leq n$, seja $\mathfrak{g}_{m}(p)$ a subálgebra de Lie de $\mathfrak{g}_{m}(n)$ gerada por $\left\{E_{i j}^{(k)} \mid i, j=1, \ldots, p ; k=0, \ldots, m-1\right\}$. Denote por $\mathcal{Z}_{m}(p)=Z\left(U\left(\mathfrak{g}_{m}(p)\right)\right)$ o centro de $U\left(\mathfrak{g}_{m}(p)\right) \subset U\left(\mathfrak{g}_{m}(n)\right)$. Temos a cadeia

$$
\mathfrak{g}_{m}(1) \subset \mathfrak{g}_{m}(2) \subset \cdots \subset \mathfrak{g}_{m}(n)
$$

de subálgebras de Lie de $\mathfrak{g}_{m}(n)$ e uma cadeia induzida

$$
U\left(\mathfrak{g}_{m}(1)\right) \subset U\left(\mathfrak{g}_{m}(2)\right) \subset \cdots \subset U\left(\mathfrak{g}_{m}(n)\right)
$$

de subálgebras de $U\left(\mathfrak{g}_{m}(n)\right)$.

A subálgebra de Gelfand-Tsetlin $\Gamma_{m}(n)$ de $\mathfrak{g}_{m}(n)$ é a subálgebras de $U\left(\mathfrak{g}_{m}(n)\right)$ gerada por $\mathcal{Z}_{m}(1), \ldots, \mathcal{Z}_{m}(n)$. Para não ter ambiguidade, denotaremos por $\bar{\zeta}_{p 1}, \bar{\zeta}_{p 2}, \ldots, \bar{\zeta}_{p, m p}$ os geradores do centro $\mathcal{Z}_{m}(p)$. Portanto, $\Gamma_{m}(n)$ é gerado pelo conjunto $\left\{\zeta_{p j} \mid p=1, \ldots, n ; j=1, \ldots, m p\right\}$.

Proposição 3.3.1. A família $\left\{\zeta_{p k} \mid p=1,2, \ldots, n, k=1,2, \ldots, m p\right\}$ é algebricamente independente, para todo $n, m \in \mathbb{Z}_{>0}$.

Demonstração. Começamos observando que $\bar{\zeta}_{p k}=\bar{F}_{11}^{(k)}+\bar{F}_{22}^{(k)}+\cdots+\bar{F}_{p p}^{(k)}$ para cada $k=1,2, \ldots, m$ e $p=1,2, \ldots, n$. Sendo assim, é suficiente mostrar que

$$
\left\{\bar{\zeta}_{p k}^{\mathbf{X}} \mid p=2, \ldots, n ; k=m+1, \ldots, m p\right\}
$$


é um conjunto algebricamente independente, onde

$$
\mathbf{X}=\left\{\bar{F}_{i i}^{(l)} \mid i=1, \ldots, n ; l=1, \ldots, m\right\} .
$$

Como cada $\bar{\zeta}_{p k}^{\mathbf{X}}$ pode ser visto como uma função polinomial em $\mathbb{C}^{m\left(n^{2}-n\right)}$ com variáveis

$$
\left\{\bar{F}_{i j}^{(l)} \mid l=1, \ldots, m \text { and } i, j=1, \ldots n, i \neq j\right\},
$$

pelo critério do Jacobiano para independência algébrica (veja [ER93, Theorem 2.3]), é suficiente mostrar que o conjunto das suas diferenciais

$$
\left\{d\left(\bar{\zeta}_{p k}^{\mathbf{X}}\right) \mid p=2, \ldots, n ; k=m+1, \ldots, m p\right\}
$$

é linearmente independente. Para isto, vamos considerar a base

$$
B=\left\{\mathbf{e}_{i j}^{(l)} \mid l=1, \ldots, m \text { and } i, j=1, \ldots n, i \neq j\right\}
$$

de $\mathbb{C}^{m\left(n^{2}-n\right)}$, sua base dual $B^{*}=\left\{f_{i j}^{(l)} \mid l=1, \ldots, m\right.$ and $\left.i, j=1, \ldots n, i \neq j\right\}$ e o ponto $v \in$ $\mathbb{C}^{m\left(n^{2}-n\right)}$, dado por

$$
f_{i j}^{(l)}(v)= \begin{cases}1 & \text { se } j=i-1, l=m \text { e } i=2,3, \ldots, n \\ 1 & \text { se } i=1, l=m \text { e } j=2,3, \ldots, n \\ 0 & \text { caso contrário. }\end{cases}
$$

Mostraremos que o conjunto $\left\{\eta_{p k} \mid p=2, \ldots, n ; k=m+1, \ldots, m p\right\}$ é linearmente independente, onde $\eta_{p k}=(-1)^{s+1} d\left(\bar{\zeta}_{p k}^{\mathbf{X}}\right)(v) \in \operatorname{Hom}_{\mathbb{C}}\left(\mathbb{C}^{m\left(n^{2}-n\right)}, \mathbb{C}\right)$ e $s \in\{1, \ldots, p\}$ é tal que $k=m(s-1)+r$ com $r \in\{1, \ldots, m\}$. Pela definição de $\bar{\zeta}_{p k}$, deduzimos que para $p=2, \ldots, n$

$$
\eta_{p k}=\sum_{i=1}^{p-1} f_{i, i+1}^{(k-m)}+\sum_{i=2}^{p} f_{i 1}^{(k-m)}
$$

quando $k=m+r$. Além disso, para $p=3,4, \ldots, n$

$$
\eta_{p k}=\sum_{i=1}^{p-s+1} f_{i, i+s-1}^{(r)}
$$

quando $k=m(s-1)+r$ e $s>2$.

Como

$$
\begin{aligned}
& \eta_{p k}= \begin{cases}f_{12}^{(k-m)}+f_{21}^{(k-m)} & \text { se } p=2 \text { e } k=m+1, \ldots, 2 m \\
\eta_{p-1, k}+f_{p-1, p}^{(k-m)}+f_{p 1}^{(k-m)} & \text { se } p=3,4, \ldots, n \text { e } k=m+1, \ldots, 2 m\end{cases} \\
& \eta_{p k}= \begin{cases}f_{1 p}^{(r)} & \text { se } p=3,4, \ldots, n ; s=p \text { e } r=1,2, \ldots, m \\
\eta_{p-1, k}+f_{p-s+1, p}^{(r)} & \text { se } p=3,4, \ldots, n ; s=3,4, \ldots, p-1 \text { e } r=1,2, \ldots, m,\end{cases}
\end{aligned}
$$

podemos concluir que os conjuntos

$$
\left\{\eta_{p k} \mid p=2,3, \ldots, n ; k=m+1, \ldots, 2 m\right\}
$$

e

$$
\left\{\eta_{p k} \mid p=3,4, \ldots, n ; k=m(s-1)+r ; s=3,4, \ldots, p ; r=1,2, \ldots, m\right\}
$$


são linearmente independentes, respectivamente. Consequentemente,

$$
\left\{\eta_{p k} \mid p=2,3, \ldots, n ; k=m+1, \ldots, m p\right\}
$$

é linearmente independente. Sendo assim, $\left\{d\left(\bar{\zeta}_{p k}^{\mathbf{X}}\right) \mid p=2, \ldots, n ; k=m+1, \ldots, m p\right\}$ é linearmente independente, seguindo que $\left\{\zeta_{p k} \mid p=1,2, \ldots, n, k=1,2, \ldots, m p\right\}$ é algebricamente independente.

Corolário 3.3.2. $\Gamma_{m}(n)$ é isomorfa à álgebra polinomial em $m n(n+1) / 2$ variáveis $\zeta_{p k}$ com $p=$ $1, \ldots, n$ e $k=1, \ldots, m p$.

Seja

$$
\mathcal{V G} \mathcal{T}_{m}(n)=V\left(\overline{\Gamma_{m}(n)}\right) \subset \mathbb{C}^{n^{2} m}
$$

a chamada variedade de Gelfand-Tsetlin determinada pelo ideal gerado por $\bar{\Gamma}_{m}(n)$ em $\bar{U}\left(\mathfrak{g}_{m}(n)\right)$. Quando $m=1$, temos a variedade de Gelfand-Tsetlin $\mathcal{V} \mathcal{G} \mathcal{T}_{n}=\mathcal{V} \mathcal{G} \mathcal{T}_{1}(n)$ para $\mathfrak{g l}_{n}(\mathbb{C})$. Pelo [Ovs03], a variedade de Gelfand-Tsetlin $\mathcal{V G} \mathcal{T}_{1}(n)$ é equidimensional de dimensão $n(n-1) / 2$. Apesar do nome variedade de Gelfand-Tsetlin, o conjunto algébrico afim $\mathcal{V G} \mathcal{T}_{m}(n)$ não é irredutível, se $n>1$. Nosso objetivo é mostrar que $\mathcal{V G} \mathcal{T}_{m}(n)$ é equidimensional de dimensão $m n(n-1) / 2$, mas veremos que isto não é verdade se $m>2$ e $n>2$.

Teorema 3.3.3. Sejam n e $m>1$ inteiros positivos. A variedade de Gelfand-Tsetlin $\mathcal{V G \mathcal { T }}{ }_{m}(n)$ é equidimensional de dimensão $m n(n-1) / 2$ se, e somente se, $n=1$ e $n=2$.

Demonstração. Primeiramente, mostraremos que a sequência $\bar{\zeta}_{p k}, k=1, \ldots, m p, p=1, \ldots, n$, é regular se $n=1$ ou $n=2$. Fixe $m>1$ e suponha $n=2$, então $\bar{\zeta}_{1 k}=\bar{F}_{11}^{(k)}$ e $\bar{\zeta}_{2 k}=\bar{F}_{11}^{(k)}+\bar{F}_{22}^{(k)}$, para cada $k=1, \ldots, m$. Portanto, $\bar{\zeta}_{p k}, k=1, \ldots, m p, p=1,2$ é regular se, e somente se, $\bar{\zeta}_{p k}^{\mathbf{X}}$, $k=1, \ldots, m p, p=1,2$, é regular, onde $\mathbf{X}=\left\{\bar{F}_{i i}^{(l)} \mid i=1,2 ; l=1, \ldots, m\right\}$. Porém, isto é verdade pelo Lema 3.2.4. Como $\bar{\zeta}_{1 k}, k=1, \ldots, m$ é uma subsequência de uma sequência regular, então também é regular. Logo, $\bar{\zeta}_{p k}, k=1, \ldots, m p, p=1, \ldots, n$, é regular se $n=1$ ou $n=2$.

Para provar a outra implicação, mostraremos que $V\left(\bar{\zeta}_{p k}, k=1, \ldots, m p, p=1, \ldots, n\right)$ não é equidimensional se $n \geq 3$. Suponha $n \geq 3$ e considere a subsequência dada por

$$
\bar{\zeta}_{1, m-1}, \bar{\zeta}_{2, m-1}, \bar{\zeta}_{3, m-1}, \bar{\zeta}_{1, m}, \bar{\zeta}_{2, m}, \bar{\zeta}_{3, m}, \bar{\zeta}_{2,2 m-1}, \bar{\zeta}_{2,2 m}, \bar{\zeta}_{3,3 m}
$$

Se $i=m-1, m$, temos que $\bar{\zeta}_{1 i}=\bar{F}_{11}^{(i)}, \bar{\zeta}_{2 i}=\bar{F}_{11}^{(i)}+\bar{F}_{22}^{(i)}$ e $\bar{\zeta}_{3 i}=\bar{F}_{11}^{(i)}+\bar{F}_{22}^{(i)}+\bar{F}_{33}^{(i)}$, então obtemos o polinômio $\bar{F}_{22}^{(i)}$ de $\bar{\zeta}_{2 i}$ se substituirmos $\bar{F}_{11}^{(i)}=0$ e obtemos o polinômio $\bar{F}_{33}^{(i)}$ de $\bar{\zeta}_{3 i}$ se substituirmos $\bar{F}_{11}^{(i)}=\bar{F}_{22}^{(i)}=0$. Pelo Lema 1.5.24, a sequência (3.9) é regular se, e somente se,

$$
\bar{\zeta}_{2,2 m}^{\mathbf{Y}}, \bar{\zeta}_{3,3 m}^{\mathbf{Y}} \operatorname{com} \mathbf{Y}=\left\{\bar{F}_{i i}^{(j)}, i=1,2,3 ; j=m-1, m\right\}
$$

é regular. Considere

$$
\begin{gathered}
y_{1}=-\bar{\zeta}_{2,2 m-1}^{\mathbf{Y}}=\bar{F}_{21}^{(m-1)} \bar{F}_{12}^{(m)}+\bar{F}_{21}^{(m)} \bar{F}_{12}^{(m-1)}, \\
y_{2}=-\bar{\zeta}_{2,2 m}^{\mathbf{Y}}=\bar{F}_{21}^{(m)} \bar{F}_{12}^{(m)}, \\
y_{3}=\bar{\zeta}_{3,3 m}^{\mathbf{Y}}=\bar{F}_{21}^{(m)} \bar{F}_{32}^{(m)} \bar{F}_{13}^{(m)}+\bar{F}_{31}^{(m)} \bar{F}_{12}^{(m)} \bar{F}_{23}^{(m)},
\end{gathered}
$$

então

$$
\begin{aligned}
V\left(y_{2}, y_{1}, y_{3}\right) & =V\left(\bar{F}_{21}^{(m)} \bar{F}_{12}^{(m)}, y_{1}, y_{3}\right) \\
& =V\left(\bar{F}_{21}^{(m)}, y_{1}, y_{3}\right) \cup V\left(\bar{F}_{12}^{(m)}, y_{1}, y_{3}\right) \\
& =V\left(\bar{F}_{21}^{(m)}, \bar{F}_{21}^{(m-1)} \bar{F}_{12}^{(m)}+\bar{F}_{21}^{(m)} \bar{F}_{12}^{(m-1)}, \bar{F}_{21}^{(m)} \bar{F}_{32}^{(m)} \bar{F}_{13}^{(m)}+\bar{F}_{31}^{(m)} \bar{F}_{12}^{(m)} \bar{F}_{23}^{(m)}\right)
\end{aligned}
$$




$$
\begin{aligned}
& \cup V\left(\bar{F}_{12}^{(m)}, \bar{F}_{21}^{(m-1)} \bar{F}_{12}^{(m)}+\bar{F}_{21}^{(m)} \bar{F}_{12}^{(m-1)}, \bar{F}_{21}^{(m)} \bar{F}_{32}^{(m)} \bar{F}_{13}^{(m)}+\bar{F}_{31}^{(m)} \bar{F}_{12}^{(m)} \bar{F}_{23}^{(m)}\right) \\
= & V\left(\bar{F}_{21}^{(m)}, \bar{F}_{21}^{(m-1)} \bar{F}_{12}^{(m)}, \bar{F}_{31}^{(m)} \bar{F}_{12}^{(m)} \bar{F}_{23}^{(m)}\right) \\
& \cup V\left(\bar{F}_{12}^{(m)}, \bar{F}_{21}^{(m)} \bar{F}_{12}^{(m-1)}, \bar{F}_{21}^{(m)} \bar{F}_{32}^{(m)} \bar{F}_{13}^{(m)}\right) \\
= & V\left(\bar{F}_{21}^{(m)}, \bar{F}_{12}^{(m)}\right) \cup V\left(\bar{F}_{21}^{(m)}, \bar{F}_{21}^{(m-1)}, \bar{F}_{31}^{(m)} \bar{F}_{12}^{(m)} \bar{F}_{23}^{(m)}\right) \\
& \cup V\left(\bar{F}_{12}^{(m)}, \bar{F}_{12}^{(m-1)}, \bar{F}_{21}^{(m)} \bar{F}_{32}^{(m)} \bar{F}_{13}^{(m)}\right) \\
= & V\left(\bar{F}_{21}^{(m)}, \bar{F}_{12}^{(m)}\right) \cup V\left(\bar{F}_{21}^{(m)}, \bar{F}_{21}^{(m-1)}, \bar{F}_{31}^{(m)}\right) \\
& \cup V\left(\bar{F}_{21}^{(m)}, \bar{F}_{21}^{(m-1)}, \bar{F}_{12}^{(m)}\right) \cup V\left(\bar{F}_{21}^{(m)}, \bar{F}_{21}^{(m-1)}, \bar{F}_{23}^{(m)}\right) \\
& \cup V\left(\bar{F}_{12}^{(m)}, \bar{F}_{12}^{(m-1)}, \bar{F}_{21}^{(m)}\right) \cup V\left(\bar{F}_{12}^{(m)}, \bar{F}_{12}^{(m-1)}, \bar{F}_{32}^{(m)}\right) \cup V\left(\bar{F}_{12}^{(m)}, \bar{F}_{12}^{(m-1)}, \bar{F}_{13}^{(m)}\right)
\end{aligned}
$$

Como $V\left(\bar{F}_{21}^{(2)}, \bar{F}_{12}^{(2)}\right)$ e $V\left(\bar{F}_{21}^{(2)}, \bar{F}_{21}^{(1)}, \bar{F}_{31}^{(2)}\right)$ estão reduzidas e não possuem a mesma dimensão, nós temos que $V\left(y_{2}, y_{1}, y_{3}\right)$ não é equidimensional. Pela Propoposição 1.5.20, $\bar{\zeta}_{2,2 m}^{\mathbf{Y}}, \bar{\zeta}_{3,3 m}^{\mathbf{Y}}$ não é regular. Pelo Lema 1.5.24, a sequência (3.9) também não é regular. Portanto, se $m>1$ e $n \geq 3, \bar{\zeta}_{p k}$, $k=1, \ldots, m p, p=1, \ldots, n$ admite uma subsequência que não é regular, implicando que ela também não é regular. Pela Proposição 1.5.20, $V\left(\bar{\zeta}_{p k}, k=1, \ldots, m p, p=1, \ldots, n\right)$ não é equidimensional se $n \geq 3$.

Aplicando o Teorema 1.5.29, temos o seguinte corolário.

Corolário 3.3.4. $U\left(\mathfrak{g}_{m}(n)\right)$ é livre como um módulo sobre $\Gamma_{m}(n)$ se $n=1$ ou $n=2$.

\subsection{Subálgebra de Bethe}

Nesta seção, considereramos outra subálgebra comutativa de $U\left(\mathfrak{g l}_{n}(\mathbb{C})[t]\right)$, que por sua vez induz subálgebras comutativas em $U\left(\mathfrak{g}_{m}(n)\right)$. A subálgebra que consideraremos é chamada subálgebra de Bethe e está relacionada ao modelo de Gaudin. Apresentaremos como ela é definida e utilizaremos o fato que é uma subálgebra comutativa, mas não provaremos isso. Para mais informações, veja [MTV06], [MTV11], [MTV14].

Para construir geradores da subálgebra de Bethe, precisaremos considerar as séries formais

$$
E_{i j}(z)=\sum_{r=0}^{\infty} E_{i j}^{(r)} z^{-r-1}, \text { onde } i, j=1, \ldots, n .
$$

Desta forma, as relações do comutador são

$$
(z-w)\left[E_{i j}(z), E_{k l}(w)\right]=\delta_{j k}\left(E_{i l}(z)-E_{i l}(w)\right)-\delta_{i l}\left(E_{k j}(z)-E_{k j}(w)\right) .
$$

Seja $\lambda=\left(\lambda_{1}, \ldots, \lambda_{n}\right)$ uma sequência de variáveis comutativas formais. Denote a álgebra de polinômios nas variáveis $\lambda_{1}, \ldots, \lambda_{n}$ com coeficientes em $U\left(\mathfrak{g l}_{n}(\mathbb{C})[t]\right)$ por $U\left(\mathfrak{g l}_{n}(\mathbb{C})[t]\right)[\lambda]$. Para uma matriz $n \times n A=\left(a_{i j}\right)$ com coeficientes em uma álgebra possivelmente não comutativa, definimos o determinante de linha por

$$
\operatorname{ldet} A=\sum_{\sigma \in S_{n}}(-1)^{\operatorname{sgn}(\sigma)} a_{1 \sigma(1)} a_{2 \sigma(2)} \ldots a_{n \sigma(n)} .
$$

Denote por $\partial_{z}$ o operador diferencial formal na variável $z$, isto é, $\partial_{z}$ é uma derivação tal que 
$\partial_{z}(z)=1$. Defina o operador universal $\mathcal{D}_{\mathcal{B}}$ pela formula

$$
\mathcal{D}_{\mathcal{B}}=\operatorname{ldet}\left(\begin{array}{cclc}
\partial_{z}-\lambda_{1}-E_{11}(z) & -E_{12}(z) & \ldots & -E_{1 n}(z) \\
-E_{21}(z) & \partial_{z}-\lambda_{2}-E_{22}(z) & \ldots & -E_{2 n}(z) \\
\vdots & \vdots & \ddots & \vdots \\
-E_{n 1}(z) & -E_{n 2}(z) & \ldots & \partial_{z}-\lambda_{n}-E_{n n}(z)
\end{array}\right)
$$

O operador universal $\mathcal{D}_{\mathcal{B}}$ é uma derivação da variável $z$ cujo os coeficientes são séries formais da variável $z^{-1}$ com coeficientes em $U\left(\mathfrak{g l}_{n}(\mathbb{C})[t]\right)[\lambda]$. Escreva

$$
\mathcal{D}_{\mathcal{B}}=\partial_{z}^{n}+\sum_{i=1}^{n} B_{i}(z) \partial_{z}^{n-i}, \quad B_{i}(z)=\sum_{j=1}^{\infty} B_{i j} z^{-j}, \quad B_{i j} \in U\left(\mathfrak{g l}_{n}(\mathbb{C})[t]\right)[\lambda] .
$$

A álgebra de Bethe é a subálgebra de $U\left(\mathfrak{g l}_{n}(\mathbb{C})[t]\right)[\lambda]$ gerada por $B_{i j}, i=1, \ldots, n, j \in \mathbb{Z}_{\geq 0}$ e será denotada por $\mathcal{B}(\lambda)$.

Proposição 3.4.1 ( [Tal06], [MTV06]). A álgebra $\mathcal{B}(\lambda)$ é comutativa. Além disso, a subálgebra $\mathcal{B}(\lambda)$ comuta com todos os elementos da subálgebra $U\left(\mathfrak{g l}_{n}(\mathbb{C})\right) \subset U\left(\mathfrak{g l}_{n}(\mathbb{C})[t]\right)[\lambda]$ e $\lambda_{i}, i=1, \ldots, n$.

Para $m \in \mathbb{Z}_{>0}$, a álgebra $\mathcal{B}(\lambda)$ induz uma subálgebra em $U\left(\mathfrak{g}_{m}(n)\right)$ que também será comutativa e denotaremos esta por $\mathcal{B}_{m}(\lambda)$. Nosso objetivo é mostrar que $U\left(\mathfrak{g}_{m}(n)\right)$ é livre como um $\mathcal{B}_{m}(\lambda)$ módulo, onde $\lambda \in \mathbb{C}^{n}$.

\subsubsection{Subálgebra de Bethe para $\mathfrak{g l}_{2}$}

Iremos considerar o caso em que $n=2$. Sejam $\lambda_{1}, \lambda_{2} \in \mathbb{C}$ tais que $\lambda_{1} \neq \lambda_{2}$. Neste caso, o operador universal será

$$
\begin{aligned}
\mathcal{D}_{\mathcal{B}} & =\left(\partial_{z}-\lambda_{1}-E_{11}(z)\right)\left(\partial_{z}-\lambda_{2}-E_{22}(z)\right)-E_{12}(z) E_{22}(z) \\
& =\partial_{z}^{2}-B_{1}(z) \partial_{z}+B_{2}(z)
\end{aligned}
$$

onde

$$
\begin{gathered}
B_{1}(z)=\lambda_{1}+\lambda_{2}+E_{11}(z)+E_{22}(z) \\
B_{2}(z)=\lambda_{1} \lambda_{2}+\lambda_{1} E_{22}(z)+\lambda_{2} E_{11}(z)+E_{11}(z) E_{22}(z)-E_{21}(z) E_{12}(z)-\partial\left(E_{22}(z)\right) .
\end{gathered}
$$

Portanto, os geradores de $B_{m}\left(\lambda_{1}, \lambda_{2}\right)$ são

$$
\begin{gathered}
B_{1 j}=E_{11}^{(j-1)}+E_{22}^{(j-1)}, \quad j=1, \ldots, m ; \\
B_{21}=\lambda_{1} E_{22}^{(0)}+\lambda_{2} E_{11}^{(0)}, \\
B_{2 j}=\lambda_{1} E_{22}^{(j-1)}+\lambda_{2} E_{11}^{(j-1)}+(j-1) E_{22}^{(j-1)}+\sum_{t+s=j-2} E_{11}^{(t)} E_{22}^{(s)}-E_{12}^{(t)} E_{21}^{(s)}, \quad j=2, \ldots, m ; \\
B_{2, m+j}=\sum_{t+s=m+j-2} E_{11}^{(t)} E_{22}^{(s)}-E_{12}^{(t)} E_{21}^{(s)}, \quad j=1, \ldots, m .
\end{gathered}
$$

Sendo assim, a imagem graduada destes elementos são

$$
\begin{gathered}
\bar{B}_{1 j}=\bar{E}_{11}^{(j-1)}+\bar{E}_{22}^{(j-1)}, \quad j=1, \ldots, m ; \\
\bar{B}_{21}=\lambda_{1} \bar{E}_{22}^{(0)}+\lambda_{2} \bar{E}_{11}^{(0)}, \\
\bar{B}_{2 j}=\sum_{t+s=j-2} \bar{E}_{11}^{(t)} E_{22}^{(s)}-\bar{E}_{12}^{(t)} E_{21}^{(s)}, \quad j=2, \ldots, 2 m ;
\end{gathered}
$$


Portanto, $\bar{B}_{1 j}=\bar{\zeta}_{j}$ e $\bar{B}_{2, m+j}=\bar{\zeta}_{m+j}$, onde $j=1, \ldots, m$ e $\zeta_{1}, \ldots, \zeta_{2 m}$ são os geradores do centro de $U\left(\mathfrak{g}_{m}(2)\right)$ introduzidos anteriormente. Iremos denotar $\mathcal{V} \mathcal{B}_{m}$ pelo conjunto algébrico associado ao ideal gerado por $\bar{B}\left(\lambda_{1}, \lambda_{2}\right)$.

Teorema 3.4.2. Se $n=2$, então o conjunto algébrico $\mathcal{V B}_{m}$ é equidimensional de dimensão $m$.

Demonstração. Para todo $m$, temos que $\bar{B}_{11}=\bar{E}_{11}^{(0)}+\bar{E}_{22}^{(0)}$ e $\bar{B}_{21}=\lambda_{1} \bar{E}_{22}^{(0)}+\lambda_{2} \bar{E}_{11}^{(0)}$. Logo, como $\lambda_{1} \neq \lambda_{2}$, temos que

$$
\begin{aligned}
\mathcal{V} \mathcal{B}_{m} & =\left(\bar{E}_{11}^{(0)}+\bar{E}_{22}^{(0)}, \lambda_{1} \bar{E}_{22}^{(0)}+\lambda_{2} \bar{E}_{11}^{(0)}, \bar{B}_{12}, \bar{B}_{13}, \ldots, \bar{B}_{1 m}, \bar{B}_{22}, \bar{B}_{23}, \ldots, \bar{B}_{2,2 m}\right) \\
& =\left(\bar{E}_{11}^{(0)}, \bar{E}_{22}^{(0)}, \bar{B}_{12}^{\mathbf{X}}, \bar{B}_{13}^{\mathbf{X}}, \ldots, \bar{B}_{1 m}^{\mathbf{X}}, \bar{B}_{22}^{\mathbf{X}}, \bar{B}_{23}^{\mathbf{X}}, \ldots, \bar{B}_{2,2 m}^{\mathbf{X}}\right)
\end{aligned}
$$

onde $\mathbf{X}=\left\{\bar{E}_{11}^{(0)}, \bar{E}_{22}^{(0)}\right\}$. Iremos considerar ambos os conjuntos como geradores do conjunto algébrico $\mathcal{V} \mathcal{B}_{m}$.

Iremos provar por indução em $m$. Suponha $m=1$, então $\bar{B}_{11}=\bar{E}_{11}^{(0)}+\bar{E}_{22}^{(0)}, \bar{B}_{21}=\lambda_{1} \bar{E}_{22}^{(0)}+$ $\lambda_{2} \bar{E}_{11}^{(0)}$ e $\bar{B}_{22}=\bar{E}_{11}^{(0)} \bar{E}_{22}^{(0)}-\bar{E}_{12}^{(0)} \bar{E}_{21}^{(0)}$. Logo, como $\lambda_{1} \neq \lambda_{2}$, temos

$$
\begin{aligned}
\mathcal{V} \mathcal{B}_{1} & =V\left(\bar{B}_{11}, \bar{B}_{21}, \bar{E}_{11}^{(0)} \bar{E}_{22}^{(0)}-\bar{E}_{12}^{(0)} \bar{E}_{21}^{(0)}\right) \\
& =V\left(\bar{E}_{11}^{(0)}, \bar{E}_{22}^{(0)}, \bar{E}_{12}^{(0)} \bar{E}_{21}^{(0)}\right) \\
& =V\left(\bar{E}_{11}^{(0)}, \bar{E}_{22}^{(0)}, \bar{E}_{12}^{(0)}\right) \cup V\left(\bar{E}_{11}^{(0)}, \bar{E}_{22}^{(0)}, \bar{E}_{21}^{(0)}\right)
\end{aligned}
$$

Assim, $\mathcal{V B}_{1}$ é equidimensional de dimensão 1.

Suponha $m>1$. Como $\bar{B}_{22}^{\mathbf{X}}=\bar{E}_{12}^{(0)} \bar{E}_{21}^{(0)}$ e $\bar{B}_{23}^{\mathbf{X}}=\bar{E}_{12}^{(0)} \bar{E}_{21}^{(1)}+\bar{E}_{12}^{(1)} \bar{E}_{21}^{(0)}$, temos que

$$
\begin{aligned}
\mathcal{V} \mathcal{B}_{m}= & V\left(\bar{E}_{11}^{(0)}, \bar{E}_{22}^{(0)}, \bar{E}_{12}^{(0)}, \bar{E}_{21}^{(0)}, \bar{B}_{13}, \bar{B}_{14}, \ldots, \bar{B}_{1 m}, \bar{B}_{24}, \bar{B}_{25}, \ldots, \bar{B}_{2,2 m}\right) \\
& \cup V\left(\bar{E}_{11}^{(0)}, \bar{E}_{22}^{(0)}, \bar{E}_{12}^{(0)}, \bar{E}_{12}^{(1)}, \bar{B}_{13}, \bar{B}_{14}, \ldots, \bar{B}_{1 m}, \bar{B}_{24}, \bar{B}_{25}, \ldots, \bar{B}_{2,2 m}\right) \\
& \cup V\left(\bar{E}_{11}^{(0)}, \bar{E}_{22}^{(0)}, \bar{E}_{21}^{(0)}, \bar{E}_{21}^{(1)}, \bar{B}_{13}, \bar{B}_{14}, \ldots, \bar{B}_{1 m}, \bar{B}_{24}, \bar{B}_{25}, \ldots, \bar{B}_{2,2 m}\right)
\end{aligned}
$$

Sejam $\mathbf{X}_{\mathbf{1}}=\left\{\bar{E}_{11}^{(0)}, \bar{E}_{22}^{(0)}, \bar{E}_{12}^{(0)}, \bar{E}_{21}^{(0)}\right\}, \mathbf{X}_{\mathbf{2}}=\left\{\bar{E}_{11}^{(0)}, \bar{E}_{22}^{(0)}, \bar{E}_{12}^{(0)}, \bar{E}_{12}^{(1)}\right\}$ e $\mathbf{X}_{\mathbf{3}}=\left\{\bar{E}_{11}^{(0)}, \bar{E}_{22}^{(0)}, \bar{E}_{21}^{(0)}, \bar{E}_{21}^{(1)}\right\}$. Denote por

$$
\mathcal{V} \mathcal{B}_{m j}=V\left(\bar{B}_{12}^{\mathbf{X}_{\mathbf{j}}}, \bar{B}_{13}^{\mathbf{X}_{\mathbf{j}}}, \ldots, \bar{B}_{1 m}^{\mathbf{X}_{\mathbf{j}}}, \bar{B}_{2,4}^{\mathbf{X}_{\mathbf{j}}}, \bar{B}_{2,4}^{\mathbf{X}_{\mathbf{j}}}, \ldots, \bar{B}_{2,2 m}^{\mathbf{X}_{\mathbf{j}}}\right),
$$

onde $j=1,2$,3. Iremos provar que cada $\mathcal{V} \mathcal{B}_{m 1}, \mathcal{V B}_{m 2}$ e $\mathcal{V} \mathcal{B}_{m 3}$ é equidimensional, assim teremos que $\mathcal{V} \mathcal{B}_{m}$ também é.

Começaremos com $\mathcal{V} \mathcal{B}_{m 1}$. Pela Proposição 1.5.20, se mostrarmos que a sequência

$$
\bar{E}_{11}^{(1)}, \bar{E}_{11}^{(1)}+\bar{E}_{22}^{(1)}, \bar{B}_{13}^{\mathbf{X}_{1}}, \bar{B}_{14}^{\mathbf{X}_{1}}, \ldots, \bar{B}_{1 m}^{\mathbf{X}_{1}}, \bar{B}_{2,4}^{\mathbf{X}_{1}}, \bar{B}_{2,5}^{\mathbf{X}_{1}}, \ldots, \bar{B}_{2,2 m}^{\mathbf{X}_{1}}
$$

é regular, então teremos que esta sequência sem o elemento $\bar{E}_{11}^{(1)}$ também é regular e $\mathcal{V} \mathcal{B}_{m 1}$ é equidimensional. Considere $\mathbf{Y}_{\mathbf{1}}=\mathbf{X}_{\mathbf{1}} \cup\left\{\bar{E}_{11}^{(1)}, \bar{E}_{22}^{(1)}\right\}$, então a sequência

$$
\bar{B}_{13}^{\mathbf{Y}_{1}}, \bar{B}_{14}^{\mathbf{Y}_{1}}, \ldots, \bar{B}_{1 m}^{\mathbf{Y}_{1}}, \bar{B}_{2,4}^{\mathbf{Y}_{1}}, \bar{B}_{2,5}^{\mathbf{Y}_{1}}, \ldots, \bar{B}_{2,2 m}^{\mathbf{Y}_{1}}
$$

é exatamente a sequência utilizada em $\mathcal{V} \mathcal{B}_{m-1}$ se fizermos a mudança de variável $\bar{E}_{p q}^{(r)} \longleftrightarrow \bar{E}_{p q}^{(r-1)}$. Portanto, pela hipótese de indução, Lema 1.5.24 e Proposição 1.5.20, a sequência (3.11) é regular. Como qualquer subsequência de (3.10) também será regular, temos que $\mathcal{V B}_{m 1}$ é equidimensional de dimensão $m$. 
Agora, considere $\mathcal{V B}_{m 2}$. Se $m=2$, basta notar que $\bar{B}_{12}^{\mathbf{X}_{2}}=\bar{E}_{11}^{(1)}+\bar{E}_{22}^{(1)}$ e $\bar{B}_{24}^{\mathbf{X}_{2}}=\bar{E}_{11}^{(1)} \bar{E}_{22}^{(1)}$. Sendo assim, temos que $\mathcal{V B}_{2,2}$ é equidimensional. Suponha $m>2$. Considere a sequência

$$
\bar{E}_{21}^{(0)}, \bar{B}_{13}^{\mathbf{X}_{2}}, \bar{B}_{14}^{\mathbf{X}_{2}}, \ldots, \bar{B}_{1 m}^{\mathbf{X}_{2}}, \bar{B}_{2,4}^{\mathbf{X}_{2}}, \bar{B}_{2,5}^{\mathbf{X}_{2}}, \ldots, \bar{B}_{2,2 m}^{\mathbf{X}_{2}}
$$

Como $\bar{B}_{12}^{\mathbf{X}_{\mathbf{2}} \cup\left\{\overline{\mathbf{E}}_{\mathbf{2 1}}^{(\mathbf{0})}\right\}}=\bar{E}_{11}^{(1)}+\bar{E}_{22}^{(1)}$ e $\bar{B}_{24}^{\mathbf{X}_{\mathbf{2}} \cup\left\{\overline{\mathbf{E}}_{\mathbf{2 1}}^{(\mathbf{0})}\right\}}=\bar{E}_{11}^{(1)} \bar{E}_{22}^{(1)}$, então podemos considerar a sequência

$$
\bar{B}_{13}^{\mathbf{Y}_{\mathbf{2}}}, \bar{B}_{14}^{\mathbf{Y}_{\mathbf{2}}}, \ldots, \bar{B}_{1 m}^{\mathbf{Y}_{\mathbf{2}}}, \bar{B}_{25}^{\mathbf{Y}_{\mathbf{2}}}, \bar{B}_{26}^{\mathbf{Y}_{\mathbf{2}}}, \bar{B}_{27}^{\mathbf{Y}_{2}}, \ldots, \bar{B}_{2,2 m}^{\mathbf{Y}_{2}}
$$

onde $\mathbf{Y}_{\mathbf{2}}=\mathbf{X}_{\mathbf{1}} \cup\left\{\bar{E}_{21}^{(0)}, \bar{E}_{11}^{(1)}, \bar{E}_{22}^{(1)}\right\}=\left\{\bar{E}_{11}^{(0)}, \bar{E}_{22}^{(0)}, \bar{E}_{12}^{(0)}, \bar{E}_{21}^{(0)}, \bar{E}_{11}^{(1)}, \bar{E}_{22}^{(1)}, \bar{E}_{12}^{(1)}\right\}$. Portanto, se considerarmos o conjunto algébrico associado à sequência anterior, obteremos

$$
\mathcal{V} \mathcal{B}_{m-1} \cap V\left(\bar{E}_{12}^{(0)}\right)
$$

se fizermos a mudança de variável $\bar{E}_{p q}^{(r)} \longleftrightarrow \bar{E}_{p q}^{(r-1)}$. Como $\bar{B}_{22}^{\mathbf{X}}=\bar{E}_{12}^{(0)} \bar{E}_{21}^{(0)}$, teremos que a sequência do caso $m-1$ continuará regular mesmo se substituirmos $\bar{B}_{22}^{\mathbf{X}}$ por $\bar{E}_{12}^{(0)}$. Sendo assim, $\mathcal{V B}_{m-1} \cap$ $V\left(\bar{E}_{12}^{(0)}\right)$ é equidimensional de dimensão $m-1$, implicando que a sequência (3.12) é regular. Pelo Lema 1.5.24 e Proposição 1.5.20, temos que $\mathcal{V B}_{m, 2}$ é equidimensional de dimensão $m$.

Analogamente ao caso anterior, podemos provar que $\mathcal{V} \mathcal{B}_{m 3}$ é equidimensional de dimensão $m$. Logo, como $\mathcal{V B}_{m 1}, \mathcal{V B}_{m 2}$ e $\mathcal{V} \mathcal{B}_{m 3}$ são equidimensionais de dimensão $m$, assim teremos que $\mathcal{V B}_{m}$ também é.

Aplicando o Teorema 1.5.29, temos o seguinte resultado.

Teorema 3.4.3. Se $\lambda_{1} \neq \lambda_{2}, U\left(\mathfrak{g}_{m}(2)\right)$ é livre sobre a subálgebra de Bethe $B_{m}\left(\lambda_{1}, \lambda_{2}\right)$.

\subsubsection{Subálgebra de Bethe para $\mathfrak{g l}_{3}$}

Temos que o operador universal $\mathcal{D}_{\mathcal{B}}$ para $\mathfrak{g l}_{3}(\mathbb{C})$ é dado por

$$
\mathcal{D}_{\mathcal{B}}=\partial_{z}^{3}-B_{1}(z) \partial_{z}^{2}-B_{2}(z) \partial_{z}-B_{3}(z),
$$

onde

$$
\begin{aligned}
& B_{1}(z)=\lambda_{1}+\lambda_{2}+\lambda_{3}+E_{11}(z)+E_{22}(z)+E_{33}(z) \\
B_{2}(z)= & \lambda_{1} \lambda_{2}+\lambda_{2} \lambda_{3}+\lambda_{1} \lambda_{3}-2 \partial_{z}\left(E_{33}(z)\right)-\partial_{z}\left(E_{22}(z)\right)-\lambda_{1}\left(E_{22}(z)+E_{33}(z)\right) \\
& -\lambda_{2}\left(E_{11}(z)+E_{33}(z)\right)-\lambda_{3}\left(E_{1}(z)+E_{22}(z)\right)-E_{11}(z) E_{22}(z)-E_{11}(z) E_{33}(z) \\
& -E_{22}(z) E_{33}(z)+E_{12}(z) E_{21}(z)+E_{13}(z) E_{31}(z)+E_{23}(z) E_{32}(z) \\
B_{3}(z)= & \lambda_{1} \lambda_{2} \lambda_{3}+\partial_{z}^{2}\left(E_{33}(z)\right)+\partial_{z}\left(E_{22}(z)\right)-\lambda_{1} \partial_{z}\left(E_{33}(z)\right)-\lambda_{3} \partial_{z}\left(E_{22}(z)\right) \lambda_{1} \partial_{z}\left(E_{33}(z)\right) \\
& -E_{11}(z) \partial_{z}\left(E_{33}(z)\right)-\partial_{z}\left(E_{22}(z)\right) E_{33}(z)-E_{22} \partial_{z}\left(E_{33}(z)\right)+\partial_{z}\left(E_{23}(z)\right) E_{32}(z) \\
& +E_{23}(z) \partial_{z}\left(E_{32}(z)\right)-E_{13}(z) \partial_{z}\left(E_{31}(z)\right)+\lambda_{1} E_{23}(z) E_{32}(z) \\
& +\lambda_{2} E_{13}(z) E_{31}(z)+\lambda_{3} E_{12}(z) E_{21}(z)+\lambda_{3} E_{12}(z) E_{21}(z)+E_{11}(z) E_{22}(z) E_{33}(z) \\
& +E_{12}(z) E_{23}(z) E_{32}(z)+E_{13}(z) E_{21}(z) E_{32}(z)-E_{11}(z) E_{23}(z) E_{32}(z) \\
& -E_{13}(z) E_{22}(z) E_{31}(z)-E_{12}(z) E_{21}(z) E_{33}(z) .
\end{aligned}
$$

Logo, pela Proposição 3.4.1, os geradores de $\bar{B}_{m}\left(\lambda_{1}, \lambda_{2}, \lambda_{3}\right)$ são

$$
\bar{B}_{1 j}=\bar{E}_{11}^{(j-1)}+\bar{E}_{22}^{(j-1)}+\bar{E}_{33}^{(j-1)}, \quad j=1, \ldots, m
$$




$$
\begin{gathered}
\bar{B}_{21}=\lambda_{1}\left(\bar{E}_{22}^{(0)}+\bar{E}_{33}^{(0)}\right)+\lambda_{2}\left(\bar{E}_{11}^{(0)}+\bar{E}_{33}^{(0)}\right)+\lambda_{3}\left(\bar{E}_{11}^{(0)}+\bar{E}_{22}^{(0)}\right) \\
\bar{B}_{31}=\lambda_{2} \lambda_{3} \bar{E}_{11}^{(0)}+\lambda_{1} \lambda_{3} \bar{E}_{22}^{(0)}+\lambda_{1} \lambda_{2} \bar{E}_{33}^{(0)} \\
\bar{B}_{32}=\lambda_{1}\left(\bar{E}_{23}^{(0)} \bar{E}_{32}^{(0)}-\bar{E}_{22}^{(0)} \bar{E}_{33}^{(0)}\right)+\lambda_{2}\left(\bar{E}_{13}^{(0)} \bar{E}_{31}^{(0)}-\bar{E}_{11}^{(0)} \bar{E}_{33}^{(0)}\right)+\lambda_{3}\left(\bar{E}_{12}^{(0)} \bar{E}_{21}^{(0)}-\bar{E}_{11}^{(0)} \bar{E}_{22}^{(0)}\right) \\
\bar{B}_{2 j}=\sum_{a+b=j-2} \bar{E}_{12}^{(a)} \bar{E}_{21}^{(b)}+\bar{E}_{13}^{(a)} \bar{E}_{31}^{(b)}+\bar{E}_{23}^{(a)} \bar{E}_{32}^{(b)}-\bar{E}_{11}^{(a)} \bar{E}_{22}^{(b)}-\bar{E}_{11}^{(a)} \bar{E}_{33}^{(b)}-\bar{E}_{22}^{(a)} \bar{E}_{33}^{(b)}, \quad j=4, \ldots, 2 m \\
\bar{B}_{3 j}=\sum_{a+b+c=j-3} \bar{E}_{11}^{(a)} \bar{E}_{22}^{(b)} \bar{E}_{33}^{(c)}+\bar{E}_{12}^{(a)} \bar{E}_{23}^{(b)} \bar{E}_{31}^{(c)}+\bar{E}_{13}^{(a)} \bar{E}_{21}^{(b)} \bar{E}_{32}^{(c)}-\bar{E}_{12}^{(a)} \bar{E}_{21}^{(b)} \bar{E}_{33}^{(c)} \\
-\bar{E}_{13}^{(a)} \bar{E}_{22}^{(b)} \bar{E}_{31}^{(c)}-\bar{E}_{11}^{(a)} \bar{E}_{23}^{(b)} \bar{E}_{32}^{(c)}, j=3, \ldots, 3 m
\end{gathered}
$$

Proposição 3.4.4. Se $\lambda_{i}=\lambda_{j}$, para algum $i, j=1,2,3$, então a sequência $\bar{B}_{r s}, r=1,2,3$, $s=1, \ldots, r m$, não é regular.

Demonstração. O sistema

$$
\begin{cases}\bar{E}_{11}^{(0)}+\bar{E}_{22}^{(0)}+\bar{E}_{33}^{(0)} & =0 \\ \lambda_{2} \lambda_{3} \bar{E}_{11}^{(0)}+\lambda_{1} \lambda_{3} \bar{E}_{22}^{(0)}+\lambda_{1} \lambda_{2} \bar{E}_{33}^{(0)} & =0 \\ \left(\lambda_{2}+\lambda_{3}\right) \bar{E}_{11}^{(0)}+\left(\lambda_{1}+\lambda_{3}\right) \bar{E}_{22}^{(0)}+\left(\lambda_{1}+\lambda_{2}\right) \bar{E}_{33}^{(0)} & =0\end{cases}
$$

terá solução única se, e somente se, $\lambda_{1} \lambda_{2}^{2}-\lambda_{1}^{2} \lambda_{2}+\lambda_{1}^{2} \lambda_{3}-\lambda_{1} \lambda_{3}^{2}+\lambda_{2} \lambda_{3}^{2}-\lambda_{2}^{2} \lambda_{3} \neq 0$. Caso este sistema não possua solução única, teremos que a sequência formada pelos geradores de $\bar{B}_{m}\left(\lambda_{1}, \lambda_{2}, \lambda_{3}\right)$ dados acima não é regular. Porém, se $\lambda_{i}=\lambda_{j}$ para algum $i, j \in\{1,2,3\}$, então $\lambda_{1} \lambda_{2}^{2}-\lambda_{1}^{2} \lambda_{2}+\lambda_{1}^{2} \lambda_{3}-$ $\lambda_{1} \lambda_{3}^{2}+\lambda_{2} \lambda_{3}^{2}-\lambda_{2}^{2} \lambda_{3}=0$. Neste caso, o sistema acima não terá solução única, implicando que a sequência $B_{r s}, r=1,2,3, s=1, \ldots, r m$, não é regular.

Portanto, para provar a regularidade da sequência formada pelos geradores de $\bar{B}_{m}\left(\lambda_{1}, \lambda_{2}, \lambda_{3}\right)$, podemos supor $\lambda_{i} \neq \lambda_{i}, i \neq j$, e poderemos considerar a seguinte sequência

$$
\begin{gathered}
\bar{E}_{11}^{(0)}, \bar{E}_{22}^{(0)}, \bar{E}_{33}^{(0)}, \\
\bar{E}_{12}^{(0)} \bar{E}_{21}^{(0)}+b \bar{E}_{23}^{(0)} \bar{E}_{32}^{(0)}, \quad \bar{E}_{13}^{(0)} \bar{E}_{31}^{(0)}+(1-b) \bar{E}_{23}^{(0)} \bar{E}_{32}^{(0)}, \\
\bar{E}_{12}^{(0)} \bar{E}_{23}^{(0)} \bar{E}_{31}^{(0)}+\bar{E}_{13}^{(0)} \bar{E}_{21}^{(0)} \bar{E}_{32}^{(0)}, \\
\bar{B}_{1 j}=\bar{E}_{11}^{(j-1)}+\bar{E}_{22}^{(j-1)}+\bar{E}_{33}^{(j-1)}, \quad j=2, \ldots, m \\
\bar{B}_{2 j}^{\mathbf{A}}=\sum_{a+b=j-2} \bar{E}_{12}^{(a)} \bar{E}_{21}^{(b)}+\bar{E}_{13}^{(a)} \bar{E}_{31}^{(b)}+\bar{E}_{23}^{(a)} \bar{E}_{32}^{(b)}-\bar{E}_{11}^{(a)} \bar{E}_{22}^{(b)}-\bar{E}_{11}^{(a)} \bar{E}_{33}^{(b)}-\bar{E}_{22}^{(a)} \bar{E}_{33}^{(b)}, \quad j=3, \ldots, 2 m, \\
\bar{B}_{3 j}^{\mathbf{A}}=\sum_{a+b+c=j-3} \bar{E}_{11}^{(a)} \bar{E}_{22}^{(b)} \bar{E}_{33}^{(c)}+\bar{E}_{12}^{(a)} \bar{E}_{23}^{(b)} \bar{E}_{31}^{(c)}+\bar{E}_{13}^{(a)} \bar{E}_{21}^{(b)} \bar{E}_{32}^{(c)}-\bar{E}_{12}^{(a)} \bar{E}_{21}^{(b)} \bar{E}_{33}^{(c)} \\
-\bar{E}_{13}^{(a)} \bar{E}_{22}^{(b)} \bar{E}_{31}^{(c)}-\bar{E}_{11}^{(a)} \bar{E}_{23}^{(b)} \bar{E}_{32}^{(c)}, j=4, \ldots, 3 m,
\end{gathered}
$$

onde $b=\left(\lambda_{2}-\lambda_{1}\right) /\left(\lambda_{3}-\lambda_{1}\right)$ e $\mathbf{A}=\left\{\bar{E}_{11}^{(0)}, \bar{E}_{22}^{(0)}, \bar{E}_{33}^{(0)}\right\}$. Como $\lambda_{i} \neq \lambda_{j}, i \neq j$, temos que $b \neq 1 \mathrm{e}$ $b \neq 0$.

Proposição 3.4.5. Se $\lambda_{i} \neq \lambda_{j}, i \neq j$, então $V\left(\bar{B}_{1}\left(\lambda_{1}, \lambda_{2}, \lambda_{3}\right)\right)$ é equidimensional de dimensão 3 .

Demonstração. Como vimos, os geradores de $\bar{B}_{1}\left(\lambda_{1}, \lambda_{2}, \lambda_{3}\right)$

$$
\bar{E}_{11}^{(0)}, \bar{E}_{22}^{(0)}, \bar{E}_{33}^{(0)},
$$




$$
\begin{gathered}
\bar{E}_{12}^{(0)} \bar{E}_{21}^{(0)}+b \bar{E}_{23}^{(0)} \bar{E}_{32}^{(0)}, \quad \bar{E}_{13}^{(0)} \bar{E}_{31}^{(0)}+(1-b) \bar{E}_{23}^{(0)} \bar{E}_{32}^{(0)} \\
\bar{E}_{12}^{(0)} \bar{E}_{23}^{(0)} \bar{E}_{31}^{(0)}+\bar{E}_{13}^{(0)} \bar{E}_{21}^{(0)} \bar{E}_{32}^{(0)}
\end{gathered}
$$

Considere a sequência maior dada por estes geradores e $\bar{E}_{12}^{(0)}-\bar{E}_{21}^{(0)}$, então basta provar a regularidade da sequência

$$
\begin{gathered}
\bar{E}_{12}^{(0)}-\bar{E}_{21}^{(0)} \\
\left(\bar{E}_{12}^{(0)}\right)^{2}+b \bar{E}_{23}^{(0)} \bar{E}_{32}^{(0)}, \quad \bar{E}_{13}^{(0)} \bar{E}_{31}^{(0)}+(1-b) \bar{E}_{23}^{(0)} \bar{E}_{32}^{(0)}, \\
\bar{E}_{12}^{(0)}\left(\bar{E}_{23}^{(0)} \bar{E}_{31}^{(0)}+\bar{E}_{13}^{(0)} \bar{E}_{32}^{(0)}\right) .
\end{gathered}
$$

Note que

$$
\begin{aligned}
& V\left(\bar{E}_{12}^{(0)}-\bar{E}_{21}^{(0)},\left(\bar{E}_{12}^{(0)}\right)^{2}+b \bar{E}_{23}^{(0)} \bar{E}_{32}^{(0)}, \bar{E}_{13}^{(0)} \bar{E}_{31}^{(0)}+(1-b) \bar{E}_{23}^{(0)} \bar{E}_{32}^{(0)}, \bar{E}_{12}^{(0)}\left(\bar{E}_{23}^{(0)} \bar{E}_{31}^{(0)}+\bar{E}_{13}^{(0)} \bar{E}_{32}^{(0)}\right)\right) \\
= & V\left(\bar{E}_{12}^{(0)}-\bar{E}_{21}^{(0)}, \bar{E}_{23}^{(0)} \bar{E}_{31}^{(0)}+\bar{E}_{13}^{(0)} \bar{E}_{32}^{(0)},\left(\bar{E}_{12}^{(0)}\right)^{2}+b \bar{E}_{23}^{(0)} \bar{E}_{32}^{(0)}, \bar{E}_{13}^{(0)} \bar{E}_{31}^{(0)}+(1-b) \bar{E}_{23}^{(0)} \bar{E}_{32}^{(0)},\right) \\
& \cup V\left(\bar{E}_{12}^{(0)}, \bar{E}_{21}^{(0)}, \bar{E}_{23}^{(0)} \bar{E}_{32}^{(0)}, \bar{E}_{13}^{(0)} \bar{E}_{31}^{(0)}+(1-b) \bar{E}_{23}^{(0)} \bar{E}_{32}^{(0)}\right) \\
= & V\left(\bar{E}_{12}^{(0)}-\bar{E}_{21}^{(0)}, \bar{E}_{23}^{(0)} \bar{E}_{31}^{(0)}+\bar{E}_{13}^{(0)} \bar{E}_{32}^{(0)},\left(\bar{E}_{12}^{(0)}\right)^{2}+b \bar{E}_{23}^{(0)} \bar{E}_{32}^{(0)}, \bar{E}_{13}^{(0)} \bar{E}_{31}^{(0)}+(1-b) \bar{E}_{23}^{(0)} \bar{E}_{32}^{(0)},\right) \\
& \cup V\left(\bar{E}_{12}^{(0)}, \bar{E}_{21}^{(0)}, \bar{E}_{23}^{(0)}, \bar{E}_{13}^{(0)}\right) \cup V\left(\bar{E}_{12}^{(0)}, \bar{E}_{21}^{(0)}, \bar{E}_{23}^{(0)}, \bar{E}_{31}^{(0)}\right) \\
& \cup V\left(\bar{E}_{12}^{(0)}, \bar{E}_{21}^{(0)}, \bar{E}_{32}^{(0)}, \bar{E}_{13}^{(0)}\right) \cup V\left(\bar{E}_{12}^{(0)}, \bar{E}_{21}^{(0)}, \bar{E}_{32}^{(0)}, \bar{E}_{31}^{(0)}\right) .
\end{aligned}
$$

Sendo assim, basta provar que

$$
\bar{E}_{12}^{(0)}-\bar{E}_{21}^{(0)}, \bar{E}_{23}^{(0)} \bar{E}_{31}^{(0)}+\bar{E}_{13}^{(0)} \bar{E}_{32}^{(0)},\left(\bar{E}_{12}^{(0)}\right)^{2}+b \bar{E}_{23}^{(0)} \bar{E}_{32}^{(0)}, \bar{E}_{13}^{(0)} \bar{E}_{31}^{(0)}+(1-b) \bar{E}_{23}^{(0)} \bar{E}_{32}^{(0)}
$$

é regular. Para isto, considere a sequência maior dada por

$$
\bar{E}_{13}^{(0)}-\bar{E}_{31}^{(0)}, \bar{E}_{12}^{(0)}-\bar{E}_{21}^{(0)}, \bar{E}_{23}^{(0)} \bar{E}_{31}^{(0)}+\bar{E}_{13}^{(0)} \bar{E}_{32}^{(0)},\left(\bar{E}_{12}^{(0)}\right)^{2}+b \bar{E}_{23}^{(0)} \bar{E}_{32}^{(0)}, \bar{E}_{13}^{(0)} \bar{E}_{31}^{(0)}+(1-b) \bar{E}_{23}^{(0)} \bar{E}_{32}^{(0)}
$$

Portanto, o conjunto algébrico da sequência 3.13 é igual a

$$
\begin{aligned}
& V\left(\bar{E}_{13}^{(0)}-\bar{E}_{31}^{(0)}, \bar{E}_{12}^{(0)}-\bar{E}_{21}^{(0)}, \bar{E}_{23}^{(0)}+\bar{E}_{32}^{(0)},\left(\bar{E}_{12}^{(0)}\right)^{2}+b \bar{E}_{23}^{(0)} \bar{E}_{32}^{(0)},\left(\bar{E}_{13}^{(0)}\right)^{2}+(1-b) \bar{E}_{23}^{(0)} \bar{E}_{32}^{(0)}\right) \\
& \cup V\left(\bar{E}_{31}^{(0)}, \bar{E}_{12}^{(0)}-\bar{E}_{21}^{(0)}, \bar{E}_{13}^{(0)},\left(\bar{E}_{12}^{(0)}\right)^{2}+b \bar{E}_{23}^{(0)} \bar{E}_{32}^{(0)}, \bar{E}_{23}^{(0)} \bar{E}_{32}^{(0)}\right) \\
= & V\left(\bar{E}_{13}^{(0)}-\bar{E}_{31}^{(0)}, \bar{E}_{12}^{(0)}-\bar{E}_{21}^{(0)}, \bar{E}_{23}^{(0)}+\bar{E}_{32}^{(0)},\left(\bar{E}_{12}^{(0)}\right)^{2}-b\left(\bar{E}_{23}^{(0)}\right)^{2},\left(\bar{E}_{13}^{(0)}\right)^{2}+(b-1)\left(\bar{E}_{23}^{(0)}\right)^{2}\right) \\
& \cup V\left(\bar{E}_{31}^{(0)}, \bar{E}_{21}^{(0)}, \bar{E}_{13}^{(0)}, \bar{E}_{12}^{(0)}, \bar{E}_{23}^{(0)}\right) \cup V\left(\bar{E}_{31}^{(0)}, \bar{E}_{21}^{(0)}, \bar{E}_{13}^{(0)}, \bar{E}_{12}^{(0)}, \bar{E}_{32}^{(0)}\right) .
\end{aligned}
$$

Como

$$
\bar{E}_{32}^{(0)}, \bar{E}_{13}^{(0)}-\bar{E}_{31}^{(0)}, \bar{E}_{12}^{(0)}-\bar{E}_{21}^{(0)}, \bar{E}_{23}^{(0)}+\bar{E}_{32}^{(0)},\left(\bar{E}_{12}^{(0)}\right)^{2}-b\left(\bar{E}_{23}^{(0)}\right)^{2},\left(\bar{E}_{13}^{(0)}\right)^{2}+(b-1)\left(\bar{E}_{23}^{(0)}\right)^{2}
$$

é regular e qualquer subsequência de uma sequência regular é regular, temos que a regularidade da sequência

$$
\bar{E}_{13}^{(0)}-\bar{E}_{31}^{(0)}, \bar{E}_{12}^{(0)}-\bar{E}_{21}^{(0)}, \bar{E}_{23}^{(0)}+\bar{E}_{32}^{(0)},\left(\bar{E}_{12}^{(0)}\right)^{2}-b\left(\bar{E}_{23}^{(0)}\right)^{2},\left(\bar{E}_{13}^{(0)}\right)^{2}+(b-1)\left(\bar{E}_{23}^{(0)}\right)^{2} .
$$


Provando que a sequência (3.13) é regular, implicando que a sequência formada pelos geradores de $\bar{B}_{1}\left(\lambda_{1}, \lambda_{2}, \lambda_{3}\right)$ também é regular. Pela Proposição 1.5.20, $V\left(\bar{B}_{1}\left(\lambda_{1}, \lambda_{2}, \lambda_{3}\right)\right)$ é equidimensional de dimensão 3 .

Aplicando o Teorema 1.5.29, temos o seguinte resultado.

Corolário 3.4.6. $U\left(\mathfrak{g l}_{3}\right)$ é um módulo livre sobre a subálgebra de Bethe $\bar{B}_{1}\left(\lambda_{1}, \lambda_{2}, \lambda_{3}\right)$, se $\lambda_{i} \neq \lambda_{j}$, $i \neq j$.

Apesar de conseguirmos escrever explicitamente os geradores de $\bar{B}_{m}\left(\lambda_{1}, \lambda_{2}, \lambda_{3}\right), m>1$, não conseguimos provar que eles formam uma sequência regular em $U\left(\mathfrak{g}_{m}(n)\right)$. Caso o leitor tenha curiosidade e queira tentar provar por si mesmo, mostraremos no próximo exemplo explicitamente os geradores para $m=2$.

Exemplo 3.4.7. Para $m=2$, temos que os elementos

$$
\begin{aligned}
& \bar{E}_{11}^{(0)}, \bar{E}_{22}^{(0)}, \bar{E}_{33}^{(0)} \text {, } \\
& \bar{E}_{11}^{(1)}+\bar{E}_{22}^{(1)}+\bar{E}_{33}^{(1)} \\
& \bar{E}_{12}^{(0)} \bar{E}_{21}^{(0)}+b \bar{E}_{23}^{(0)} \bar{E}_{32}^{(0)}, \quad \bar{E}_{13}^{(0)} \bar{E}_{31}^{(0)}+(1-b) \bar{E}_{23}^{(0)} \bar{E}_{32}^{(0)}, \\
& \bar{E}_{12}^{(0)} \bar{E}_{23}^{(0)} \bar{E}_{31}^{(0)}+\bar{E}_{13}^{(0)} \bar{E}_{21}^{(0)} \bar{E}_{32}^{(0)} \text {, } \\
& \bar{B}_{23}^{\mathbf{A}}=\bar{E}_{12}^{(0)} \bar{E}_{21}^{(1)}+\bar{E}_{12}^{(1)} \bar{E}_{21}^{(0)}+\bar{E}_{13}^{(0)} \bar{E}_{31}^{(1)}+\bar{E}_{13}^{(1)} \bar{E}_{31}^{(0)}+\bar{E}_{23}^{(0)} \bar{E}_{32}^{(1)}+\bar{E}_{23}^{(1)} \bar{E}_{32}^{(0)}, \\
& \bar{B}_{34}^{\mathbf{A}}=\bar{E}_{12}^{(0)} \bar{E}_{23}^{(0)} \bar{E}_{31}^{(1)}+\bar{E}_{12}^{(0)} \bar{E}_{23}^{(1)} \bar{E}_{31}^{(0)}+\bar{E}_{12}^{(1)} \bar{E}_{23}^{(0)} \bar{E}_{31}^{(0)} \\
& +\bar{E}_{13}^{(0)} \bar{E}_{21}^{(0)} \bar{E}_{32}^{(1)}+\bar{E}_{13}^{(0)} \bar{E}_{21}^{(1)} \bar{E}_{32}^{(0)}+\bar{E}_{13}^{(1)} \bar{E}_{21}^{(0)} \bar{E}_{32}^{(0)} \\
& -\bar{E}_{12}^{(0)} \bar{E}_{21}^{(0)} \bar{E}_{33}^{(1)}-\bar{E}_{13}^{(0)} \bar{E}_{22}^{(1)} \bar{E}_{31}^{(0)}-\bar{E}_{11}^{(1)} \bar{E}_{23}^{(0)} \bar{E}_{32}^{(0)} \text {, } \\
& \bar{B}_{24}^{\mathbf{A}}=\bar{E}_{12}^{(1)} \bar{E}_{21}^{(1)}+\bar{E}_{13}^{(1)} \bar{E}_{31}^{(1)}+\bar{E}_{23}^{(1)} \bar{E}_{32}^{(1)}-\bar{E}_{11}^{(1)} \bar{E}_{22}^{(1)}-\bar{E}_{11}^{(1)} \bar{E}_{33}^{(1)}-\bar{E}_{22}^{(1)} \bar{E}_{33}^{(1)}, \\
& \bar{B}_{35}^{\mathbf{A}}=\bar{E}_{12}^{(0)} \bar{E}_{23}^{(1)} \bar{E}_{31}^{(1)}+\bar{E}_{12}^{(1)} \bar{E}_{23}^{(0)} \bar{E}_{31}^{(1)}+\bar{E}_{12}^{(1)} \bar{E}_{23}^{(1)} \bar{E}_{31}^{(0)} \\
& +\bar{E}_{13}^{(0)} \bar{E}_{21}^{(1)} \bar{E}_{32}^{(1)}+\bar{E}_{13}^{(1)} \bar{E}_{21}^{(0)} \bar{E}_{32}^{(1)}+\bar{E}_{13}^{(1)} \bar{E}_{21}^{(1)} \bar{E}_{32}^{(0)} \\
& -\left(\bar{E}_{12}^{(0)} \bar{E}_{21}^{(1)}+\bar{E}_{12}^{(1)} \bar{E}_{21}^{(0)}\right) \bar{E}_{33}^{(1)}-\left(\bar{E}_{13}^{(0)} \bar{E}_{31}^{(1)}+\bar{E}_{13}^{(1)} \bar{E}_{31}^{(0)}\right) \bar{E}_{22}^{(1)}-\left(\bar{E}_{23}^{(0)} \bar{E}_{32}^{(1)}+\bar{E}_{23}^{(1)} \bar{E}_{32}^{(0)}\right) \bar{E}_{11}^{(1)} \text {, } \\
& \bar{B}_{36}^{\mathbf{A}}=\bar{E}_{11}^{(1)} \bar{E}_{22}^{(1)} \bar{E}_{33}^{(1)}+\bar{E}_{12}^{(1)} \bar{E}_{23}^{(1)} \bar{E}_{31}^{(1)}+\bar{E}_{13}^{(1)} \bar{E}_{21}^{(1)} \bar{E}_{32}^{(1)}-\bar{E}_{12}^{(1)} \bar{E}_{21}^{(1)} \bar{E}_{33}^{(1)} \\
& -\bar{E}_{13}^{(1)} \bar{E}_{22}^{(1)} \bar{E}_{31}^{(1)}-\bar{E}_{11}^{(1)} \bar{E}_{23}^{(1)} \bar{E}_{32}^{(1)},
\end{aligned}
$$

são geradores de $\bar{B}_{2}\left(\lambda_{1}, \lambda_{2}, \lambda_{3}\right)$. 


\section{Referências Bibliográficas}

[AM69] Michael Francis Atiyah e Ian Grant Macdonald. Introduction to commutative algebra. Addison-Wesley Publishing Co., Reading, Mass.-London-Don Mills, Ont., 1969. 7, 8, 38, 64

[Ben20] Germán Benitez. Gelfand-Tsetlin varieties for $\mathfrak{g l}_{n}$. Internat. J. Algebra Comput., 30(7):1485-1504, 2020. 90

[BLL15] Daniel Britten, Michael Lau e Frank Lemire. Weight modules for current algebras. J. Algebra, 440:245-263, 2015. 2, 5, 29, 32, 45, 46, 50, 53, 63, 64, 67, 69, 71, 73

[Blo81] Richard E. Block. The irreducible representations of the Lie algebra $\mathfrak{s l}_{2}$ and of the Weyl algebra. Adv. in Math., 39(1):69-110, 1981. 1

[Bou72] Nicolas Bourbaki. Éléments de mathématique. Fasc. XXXVII. Groupes et algèbres de Lie. Chapitre II: Algèbres de Lie libres. Chapitre III: Groupes de Lie. Hermann, Paris, 1972. Actualités Scientifiques et Industrielles, No. 1349. 19

[Bre95] Murray Bremner. Four-point affine Lie algebras. Proc. Amer. Math. Soc., 123(7):19811989, 1995. 1, 48

[BT15] Herivelto Borges e Eduardo Tengan. Álgebra Comutativa em Quatro Movimentos. Projeto Euclides. IMPA, 2015. 7, 38, 63

[CG05] Vyjayanthi Chari e Jacob Greenstein. An application of free Lie algebras to polynomial current algebras and their representation theory. Em Infinite-dimensional aspects of representation theory and applications, volume 392 of Contemp. Math., páginas 15-31. Amer. Math. Soc., Providence, RI, 2005. 1

[CG07] Vyjayanthi Chari e Jacob Greenstein. Current algebras, highest weight categories and quivers. Adv. Math., 216(2):811-840, 2007. 1

[Cha86] Vyjayanthi Chari. Integrable representations of affine Lie-algebras. Invent. Math., 85(2):317-335, 1986. 1

[CP86] Vyjayanthi Chari e Andrew Pressley. New unitary representations of loop groups. Math. Ann., 275(1):87-104, 1986. 1

[CP87] Vyjayanthi Chari e Andrew Pressley. A new family of irreducible, integrable modules for affine Lie algebras. Math. Ann., 277(3):543-562, 1987. 1

[CP88] Vyjayanthi Chari e Andrew Pressley. Integrable representations of twisted affine Lie algebras. J. Algebra, 113(2):438-464, 1988. 1

[DG09] Ivan Dimitrov e Dimitar Grantcharov. Classification of simple weight modules over affine lie algebras, 2009. 45 
[Dix96] Jacques Dixmier. Algèbres enveloppantes. Les Grands Classiques Gauthier-Villars. [Gauthier-Villars Great Classics]. Éditions Jacques Gabay, Paris, 1996. Reprint of the 1974 original. $26,27,43$

[Dri85] Vladimir Gershonovich Drinfeld. Hopf algebras and the quantum Yang-Baxter equation. Dokl. Akad. Nauk SSSR, 283(5):1060-1064, 1985. 85

[Eis95] David Eisenbud. Commutative algebra, volume 150 of Graduate Texts in Mathematics. Springer-Verlag, New York, 1995. With a view toward algebraic geometry. 7, 9, 38, 39

[ER93] Richard Ehrenborg e Gian-Carlo Rota. Apolarity and canonical forms for homogeneous polynomials. European J. Combin., 14(3):157-181, 1993. 88, 104

[ER01] S. Eswara Rao. Classification of irreducible integrable modules for multi-loop algebras with finite-dimensional weight spaces. J. Algebra, 246(1):215-225, 2001. 1

[ERB17] S. Eswara Rao e Punita Batra. Classification of irreducible integrable highest weight modules for current Kac-Moody algebras. J. Algebra Appl., 16(7):1750123, 10, 2017. 46, 49,79

[Fer90] S. L. Fernando. Lie algebra modules with finite-dimensional weight spaces. I. Trans. Amer. Math. Soc., 322(2):757-781, 1990. 45, 55, 56

[FO05] Vyacheslav Futorny e Serge Ovsienko. Kostant's theorem for special filtered algebras. Bull. London Math. Soc., 37(2):187-199, 2005. 3, 5, 42, 43, 83

[Geo94a] François Geoffriau. Sur le centre de l'algèbre enveloppante d'une algèbre de Takiff. Ann. Math. Blaise Pascal, 1(2):15-31 (1995), 1994. 1, 90

[Geo94b] François Geoffriau. Une propriété des algèbres de Takiff. C. R. Acad. Sci. Paris Sér. I Math., 319(1):11-14, 1994. 1, 43, 90

[Geo95] François Geoffriau. Homomorphisme de Harish-Chandra pour les algèbres de Takiff généralisées. J. Algebra, 171(2):444-456, 1995. 1

[GLZ11] Xiangqian Guo, Rencai Lu e Kaiming Zhao. Simple Harish-Chandra modules, intermediate series modules, and Verma modules over the loop-Virasoro algebra. Forum Math., 23(5):1029-1052, 2011. 45

[GM62] Murray Gell-Mann. Symmetries of baryons and mesons. Phys. Rev., 125:1067-1084, Feb 1962. 1

[GM64] Murray Gell-Mann. The symmetry group of vector and axial vector currents. Physics Physique Fizika, 1:63-75, Jul 1964. 1

[Har07] Brian Hartwig. The tetrahedron algebra and its finite-dimensional irreducible modules. Linear Algebra Appl., 422(1):219-235, 2007. 1

[HS97] Peter John Hilton e Urs Stammbach. A course in homological algebra, volume 4 of Graduate Texts in Mathematics. Springer-Verlag, New York, second edição, 1997. 13

[Hum78] James Edward Humphreys. Introduction to Lie algebras and representation theory, volume 9 of Graduate Texts in Mathematics. Springer-Verlag, New York-Berlin, 1978. Second printing, revised. 9, 11, 12, 14, 15, 16, 18, 19, 25, 34, 38

[Isa09] Irving Martin Isaacs. Algebra: a graduate course, volume 100 of Graduate Studies in Mathematics. American Mathematical Society, Providence, RI, 2009. Reprint of the 1994 original. 28 
[Jac45] Nathan Jacobson. Structure theory of simple rings without finiteness assumptions. Trans. Amer. Math. Soc., 57:228-245, 1945. 28

[Jim85] Michio Jimbo. A $q$-difference analogue of $U(\mathfrak{g})$ and the Yang-Baxter equation. Lett. Math. Phys., 10(1):63-69, 1985. 85

[Kas84] Christian Kassel. Kähler differentials and coverings of complex simple Lie algebras extended over a commutative algebra. Em Proceedings of the Luminy conference on algebraic K-theory (Luminy, 1983), volume 34, páginas 265-275, 1984. 2, 45, 49

[KN87] Igor Moiseevich Krichever e S. P. Novikov. Algebras of Virasoro type, Riemann surfaces and the structures of soliton theory. Funktsional. Anal. $i$ Prilozhen., 21(2):46-63, 1987. 1,48

[Kos63] Bertram Kostant. Lie group representations on polynomial rings. Amer. J. Math., 85:327404, 1963. 38, 43, 83

[Lau10] Michael Lau. Representations of multiloop algebras. Pacific J. Math., 245(1):167-184, 2010. 1

[Lau14] Michael Lau. Representations of twisted current algebras. J. Pure Appl. Algebra, 218(11):2149-2163, 2014. 1, 48

[Lau18] Michael Lau. Classification of Harish-Chandra modules for current algebras. Proc. Amer. Math. Soc., 146(3):1015-1029, 2018. 2, 45, 46, 55, 59, 76, 77

[Mat87] H. Matsumura. Commutative Ring Theory. Cambridge Studies in Advanced Mathematics. Cambridge University Press, 1987. 38, 42

[Mat92] Olivier Mathieu. Classification of Harish-Chandra modules over the Virasoro Lie algebra. Invent. Math., 107(2):225-234, 1992. 2, 45

[Mat00] Olivier Mathieu. Classification of irreducible weight modules. Ann. Inst. Fourier (Grenoble), 50(2):537-592, 2000. 1, 45

[Mic89] Jouko Mickelsson. Current Algebras and Groups. Plenum Monographs in Nonlinear Physics. Springer US, 1989. 1

[Mol97] Alexander Molev. Casimir elements for certain polynomial current lie algebras. Em Group 21, Physical Applications and Mathematical Aspects of Geometry, Groups, and Algebras, volume 1, páginas 172-176. World Scientific, 1997. 3, 83, 84, 85

[Mol07] Alexander Molev. Yangians and classical Lie algebras, volume 143 of Mathematical Surveys and Monographs. American Mathematical Society, Providence, RI, 2007. 85, 86, 88

[MP95] Robert Vaughan Moody e Arturo Pianzola. Lie Algebras with Triangular Decompositions. Wiley-Interscience and Canadian Mathematics Series of Monographs and Texts. Wiley, 1995. 29

[MS19] Tiago Macedo e Alistair Savage. Invariant polynomials on truncated multicurrent algebras. J. Pure Appl. Algebra, 223(1):349-368, 2019. 1

[MTV06] Evgeny Mukhin, Vitaly Tarasov e Alexander Varchenko. Bethe eigenvectors of higher transfer matrices. J. Stat. Mech. Theory Exp., (8):P08002, 44, 2006. 3, 106, 107

[MTV11] Evgeny Mukhin, Vitaly Tarasov e Alexander Varchenko. Bethe algebra of the $\mathfrak{g l}_{N+1}$ Gaudin model and algebra of functions on the critical set of the master function. Em New trends in quantum integrable systems, páginas 307-324. World Sci. Publ., Hackensack, NJ, 2011. 106 
[MTV14] Evgeny Mukhin, Vitaly Tarasov e Alexander Varchenko. Bethe algebra of Gaudin model, Calogero-Moser space, and Cherednik algebra. Int. Math. Res. Not. IMRN, (5):11741204, 2014. 106

[Mus01] Mircea Mustaţă. Jet schemes of locally complete intersection canonical singularities. Invent. Math., 145(3):397-424, 2001. Com um apêndice de David Eisenbud and Edward Frenkel. 43

[MZ07] Volodymyr Mazorchuk e Kaiming Zhao. Classification of simple weight Virasoro modules with a finite-dimensional weight space. J. Algebra, 307(1):209-214, 2007. 45

[NSS12] Erhard Neher, Alistair Savage e Prasad Senesi. Irreducible finite-dimensional representations of equivariant map algebras. Trans. Amer. Math. Soc., 364(5):2619-2646, 2012. 1,48

[Ovs03] Serge Ovsienko. Strongly nilpotent matrices and Gelfand-Zetlin modules. volume 365, páginas 349-367. 2003. Special issue on linear algebra methods in representation theory. 3,105

[PB10] Tanusree Pal e Punita Batra. Representations of graded multiloop Lie algebras. Comm. Algebra, 38(1):49-67, 2010. 1

[Qui69] Daniel Quillen. On the endomorphism ring of a simple module over an enveloping algebra. Proc. Amer. Math. Soc., 21:171-172, 1969. 22

[RT92] Mustapha Rais e Patrice Tauvel. Indice et polynômes invariants pour certaines algèbres de Lie. J. Reine Angew. Math., 425:123-140, 1992. 1, 43, 84, 89

[Sav12] Alistair Savage. Classification of irreducible quasifinite modules over map Virasoro algebras. Transform. Groups, 17(2):547-570, 2012. 45

[SM10] Luiz Antonio Barrera San Martin. Álgebras de Lie. Editora Unicamp, 2010. 9, 14, 16

[Tak71] S. J. Takiff. Rings of invariant polynomials for a class of Lie algebras. Trans. Amer. Math. Soc., 160:249-262, 1971. 1

[Tal06] Dmitry Talalaev. The quantum Gaudin system. Funktsional. Anal. i Prilozhen., 40(1):8691, 2006. 3, 107

[TJG72] Sam Treiman, Roman Jackiw e David J. Gross. Lectures on Current Algebra and Its Applications. Princeton Series in Physics. Princeton University Press, 1972. 1

[Wil11] Benjamin J. Wilson. Highest-weight theory for truncated current Lie algebras. J. Algebra, 336:1-27, 2011. 1

[ZS76] Oscar Zariski e Pierre Samuel. Commutative Algebra II. Graduate Texts in Mathematics. Springer New York, 1976. 38, 41 